\title{
Towards improved guidelines for cost evaluation of carbon capture and storage
}

\author{
A white paper prepared by
}

Simon Roussanaly ${ }^{\mathrm{a}, *}$, Edward S. Rubin ${ }^{\mathrm{b}}$, Mijndert van der Spek ${ }^{\mathrm{c}}$, George Booras ${ }^{\mathrm{d}}$, Niels Berghout ${ }^{\mathrm{e}}$, Tim Fout ${ }^{\mathrm{f}}$, Monica Garcia ${ }^{\mathrm{g}}$, Stefania Gardarsdottir ${ }^{\mathrm{a}}$, Vishalini Nair Kuncheekanna ${ }^{\text {h }}$, Michael Matuszewski ${ }^{\mathrm{i}}$, Sean McCoy ${ }^{\mathrm{j}}$, Joshua Morgan ${ }^{\mathrm{i}}$, Shareq Mohd Nazirk, Andrea Ramirez

Edited by Simon Roussanaly, Edward S. Rubin, Mijndert van der Spek

\author{
${ }^{a}$ SINTEF Energy Research, Trondheim, Norway \\ ${ }^{\mathrm{b}}$ Carnegie Mellon University, Pittsburgh, PA, United States \\ ${ }^{\mathrm{c}}$ Heriot-Watt University, Edinburgh, United Kingdom \\ ${ }^{\mathrm{d}}$ Electric Power Research Institute, Palo Alto, CA, United States \\ ${ }^{\mathrm{e}}$ International Energy Agency, Paris, France \\ ${ }^{\mathrm{f}}$ National Energy Technology Laboratory, Morgantown, WV, United States \\ ${ }^{g}$ IEAGHG, Cheltenham, United Kingdom \\ ${ }^{\mathrm{h}}$ NTNU, Trondheim, Norway \\ ${ }^{i}$ National Energy Technology Laboratory, Pittsburgh, PA, United States \\ ${ }^{\mathrm{j}}$ University of Calgary, Calgary, Canada \\ ${ }^{\mathrm{k}}$ KTH Royal Institute of Technology, Stockholm, Sweden \\ ${ }^{1}$ Delft University of Technology, Delft, The Netherlands \\ * Corresponding author. e-mail address: simon.roussanaly@sintef.no
}

March 2021 


\section{Disclaimer}

All authors listed on the title page contributed to one or more chapters of this white paper; authors of individual chapters are listed in a footnote to each chapter. We note that co-authorship does not necessarily imply a unanimous consensus or endorsement by all individual authors of all content of the white paper. Nor do the findings, opinions, conclusions and recommendations presented in this paper necessarily reflect the views of the authors' organisations and/or their members.

\section{Acknowledgements}

Support for the first chapter of this white paper was provided, in part, by a subcontract to Carnegie Mellon University from KeyLogic Systems, Inc. under Contract No. DE-FE0025912 from the U.S. Department of Energy's National Energy Technology Laboratory. The authors would also like to thank Rahul Anantharaman, Chao Fu, Jabir Ali Ouassou, Mari Voldsund, Chris Greig and Ann Aalberg for their helpful comments and inputs. 


\section{Executive summary}

Understanding the costs of carbon capture and storage (CCS) is essential to understand the role for and potential of CCS technology in addressing climate change, for guidance in research activities aiming to reduce the cost and improve the performance of promising new CCS technologies in different applications. In practice, however, there are many challenges in establishing reliable cost estimates for CCS technologies. To help identify and overcome these challenges, a group of experts from industry, government, academia and other organisations came together in 2011 to form the CCS Cost Network (which came under the aegis of IEAGHG in 2017 [1]).

Following discussions at the first CCS Cost Network workshop [1], several members of the workshop steering committee formed a task force to focus on the basic structure of CCS cost estimates. That effort produced a White Paper entitled, "Toward a Common Method of Cost Estimation for CCS at Fossil Fuel Power Plants" [2]. This white paper aimed at overcoming identified pitfalls in CCS cost evaluations for fossil fuel power plants arising from the different methodologies used by various organisations. Towards this aim, the white paper established a common costing methodology and nomenclature, as well as guidelines for CCS cost reporting to improve the clarity and consistency of cost estimates for greenhouse gas mitigation measures.

While that work laid the foundation for establishing a common costing methodology for CCS, several important cost issues still remained to be addressed. Building on that earlier work and the interest from additional organisations, the current white paper is an effort to draw up a complementary set of CCS costing guidelines in three complementary areas where further guidelines and better practices are needed, and where efforts are underway to address those topics. This effort is a collaboration among researchers at several industrial research institutes (Electric Power Research Institute, SINTEF Energy Research), universities (Carnegie Mellon University, Delft University of Technology, Heriot-Watt University, KTH Royal Institute of Technology, NTNU, University of Calgary), governmental laboratories (NETL), intergovernmental organisation (IEA), and international organisation (IEAGHG).

The first area of study tackles the establishment of improved guidelines for cost evaluation of advanced low-carbon technology (such as a new $\mathrm{CO}_{2}$ capture process or a novel power plant design). While emerging technologies may have the potential to reduce the future cost of $\mathrm{CO}_{2}$ capture, most techno-economic assessments of emerging technologies are performed considering them as mature. This bias introduces significant challenges to the reliable comparison of technologies at different stages of development, or with different concepts of a particular technology. To address this issue, we develop a framework for estimating the future $N^{\text {th }}$-of-a-kind (NOAK) cost of advanced low-carbon technologies that are currently at early pre-commercial stages of development. This framework addresses the two types of questions that commonly motivate a cost analysis (called "What If" and "What Will" questions). In addition, we address shortcomings in the classic "bottom-up" engineering-economic method currently used to estimate NOAK costs. We further describe a hybrid costing method that combines a bottom-up analysis of the first-of-a-kind (FOAK) commercial cost of an advanced technology with an empirical model employing experience curves to project its future cost.

The second area of study focuses on CCS from non-power industries (such as cement plants, steel mills, refineries, and other industrial sources of $\mathrm{CO}_{2}$ emissions), which is a growing area of focus for CCS implementation. There are key challenges and factors that are unique to this sector and have a large impact on the cost evaluations of CCS from such facilities but are often overlooked or insufficiently addressed. These include cost metrics (especially in the context of industrial plants 
with multiple output products), energy supply aspects, retrofitting costs, maturity of the capture technology, and $\mathrm{CO}_{2}$ transport and storage cost. We thus seek to develop a set of recommendations to better include and treat these aspects in cost evaluation of CCS in industrial applications. Where possible, examples are given to demonstrate quantitative impact and show how costs may vary widely on a case-by-case basis.

The final area addresses quality assurance and uncertainty evaluations of data and models used in CCS cost analysis. Quantifying the effect of such uncertainties on techno-economic analyses of CCS technologies and systems is critical for proper interpretation and communication of results. As many CCS technologies are novel concepts in early stages of development, substantial uncertainties exist in their performance and cost. Although uncertainty analysis itself is not novel, with some additional methods already frequently used by the CCS techno-economic analysis community, a document that provides a comprehensive overview of methods and approaches, as well as guidance on their selection and use, was still lacking. Given its importance, we seek to fill this gap by providing a critical review of uncertainty analysis methods along with guidance on the selection and use of these methods for CCS techno-economic analyses, highlighting good practice and examples from the CCS literature.

This white paper presents a new set of guidelines developed to address important cost issues in each of these three areas. The paper is organised into three chapters each tackling one of these areas: Chapter 1: Towards improved cost guidelines for advanced low-carbon technologies; Chapter 2: Towards improved cost evaluation of carbon capture and storage from industry; Chapter 3: Toward improved guidelines for uncertainty analysis of carbon capture and storage technoeconomic studies. While more detail can be found in each chapter and the corresponding published papers [3-5], a summary is provided below for each topic.

\section{Towards improved cost guidelines for advanced low-carbon technologies}

This chapter presents a framework for estimating the future " $N$ th-of-a-kind" (NOAK) cost of advanced technologies that are currently at early pre-commercial stages of development. That framework distinguishes between two types of question that commonly motivate such a cost analysis: "What If" questions about the hypothetical cost of a technology that meets specified R\&D goals; and "What Will" questions regarding the actual expected cost of an advanced technology once it is mature.

The latter type of question is of particular interest because of the shortcomings in current methods for estimating the expected NOAK cost that are identified, as well as the ambiguity in many cost studies as to the meaning of reported NOAK costs. Indeed, the first conclusion drawn from this study is that there is a need for greater clarity as to the question a reported NOAK cost result is intended to answer. Going forward, we recommend that authors of cost studies use the term "NOAK goal" or "aspirational NOAK" when conducting "What If" studies, and the term "expected NOAK" or "projected NOAK" when reporting "What Will" estimates of the future cost of an advanced (pre-commercial) technology. While some authors may prefer to use other adjectives to describe study goals, the term NOAK should not be used in isolation without a clear descriptor.

Regarding "What Will" cost estimates for advanced technologies, a hybrid costing method, which combines a "bottom-up" engineering-economic analysis with a "top-down" model of technological learning, offers an approach to address the identified deficiencies in current methods. 
The hybrid cost analysis focuses new attention on the current (first-of-a-kind) commercial cost of an advanced technology. Methods and assumptions for estimating FOAK plant costs, which vary significantly from those for known commercial technologies are discussed. A bottom-up FOAK cost estimate is recommended as the starting point for projecting future cost reductions of advanced technologies based on historical experience with similar or related technologies. This is embodied in the application of technology experience curves and historical learning rates that underscore the importance of cumulative experience in plant deployments and operation (together with other factors) in achieving future cost reductions. Methods for implementing these cost models are also elaborated and illustrated. Additional discussions also provide guidelines for the selection of appropriate values of performance and cost parameters for "What If" studies of aspirational NOAK costs for advanced technologies.

As with traditional cost analyses, a variety of tools and methods are available to also characterize uncertainties in cost results and the influence of key decision variables. Three methods, sensitivity analysis, probabilistic analysis, and expert judgments, are discussed and illustrated. An uncertainty analysis is especially important for FOAK and projected NOAK costs for advanced technologies because of their intrinsically greater uncertainty compared to well-established commercial technologies. Uncertainty analysis also is important in calculating aspirational NOAK cost goals since these also depend heavily on assumptions and judgments that may vary considerably.

This chapter represents an initial effort to identify needed improvements in current costing methods and to provide guidance for future cost studies, especially for advanced (pre-commercial) technologies. Continuing efforts also are needed to further develop the models, databases, and guidelines that support applications of the hybrid costing method. This includes methodological improvements and quantitative guidelines to improve the development of FOAK cost estimates and reduce the uncertainties in projected NOAK costs for advanced technologies. Despite current limitations, however, the hybrid modelling approach can enhance prevailing assessments of advanced technologies by providing new insights regarding the time frame and requirements for their cost-competitiveness with existing or other advanced technology concepts. Further applications of the hybrid method can thus begin to offer a more complete and realistic approach to assessing the economic potential of advanced energy, industrial and environmental systems, and priorities for R\&D management.

\section{Towards improved cost evaluation of carbon capture and storage from industry}

While extensive studies have investigated the techno-economic performance of CCS applied to industrial sources, wide differences in cost estimates have been observed. While this is due in part to differences in the cases studied and the choice of capture technology, a significant part arises from aspects related to cost assessment methods and assumptions. Building on a previous CCS costing guideline paper [6], this chapter aims to contribute to the development of improved guidelines for cost evaluation of CCS from industrial applications. The following key messages and recommendations can be extracted from the work:

- Several publicly available, transparent, and detailed techno-economic studies exist for different industrial sectors (e.g. iron and steel, cement, refinery, hydrogen, ammonia/urea and methanol, pulp and paper). These studies provide a high level of technical and cost details on the industrial facilities considered, which can be used to strengthen future evaluation of CCS from such facilities. Furthermore, these studies have also performed detailed evaluations of currently available $\mathrm{CO}_{2}$ capture technologies, which can be used as a base case in comparative 
assessments involving new technologies. However, it is worth noting that most of these detailed studies are based on European locations and that some industry sectors are not yet studied in sufficient detail and where more specific benchmarks need to be developed in the coming years (e.g. waste-to-energy, offshore oil and gas production facilities, petrochemicals and others).

- The same basic cost metrics used for CCS from power plants are relevant to industrial processes, although in some cases these may be calculated differently. Furthermore, a key challenge that might arise in the calculation of cost metrics for industrial plants is that many processes result in multiple products. In such cases, the cost of CCS may need to be allocated across these products when reporting costs on a normalised basis (e.g., cost per unit of product). While different allocation approaches exist to distribute these (or other) costs, which differentiate between joint and separable costs and between products and by-products, there is no standardised methodology currently in use. Thus, it is recommended to report CCS costs using more than one allocation method as this will provide insights into the impact of different methods on cost performance.

- The origin and production/supply strategy of the steam and electricity required for the $\mathrm{CO}_{2}$ capture process may vary considerably on a case-by-case basis, and thus have a significant impact on overall cost, associated $\mathrm{CO}_{2}$ emissions, and the $\mathrm{CO}_{2}$ avoidance cost. It is recommended that transparent scenarios of realistic (future) heat and power supply strategies be included in cost evaluations and that costing methods and assumptions be explicitly reported. Although this recommendation seems straightforward, it is not common to find cost assessments in literature with high level of transparency, both in assumptions and data.

- The energy supply strategy and the cost and associated emissions intensity of heat and power supplies can also be site-specific and depends on parameters such as energy prices, which can change significantly over time. To understand the impact of these uncertainties, analysts are encouraged to use scenarios for plausible combinations of future energy and carbon prices, so as to clearly understand the impact of possible outcomes.

- To date, few studies properly account for the cost of retrofitting $\mathrm{CO}_{2}$ capture at existing facilities. As illustrated in the literature, these costs can vary considerably on a case-by-case basis. Thus, retrofit costs and assumptions should be properly accounted for and documented in studies considering retrofit applications of CCS from industry. Particular attention should be paid to the following aspects: economic impact of potentially required plant production stoppages, impacts on the main output product quality and plant operation, flue gas treatment requirements, spatial constraints in plant sites, flue gas interconnection, and utilities connection costs.

- Costs associated with $\mathrm{CO}_{2}$ transport and storage are often assumed to be a fixed unit cost per tonne of $\mathrm{CO}_{2}$, independent of the expected transport and storage conditions (distance, volume, and type of transport and storage). While there is significant room to improve the quality of transport and storage cost estimates, it is recommended that any such estimates be based on at least the applicable $\mathrm{CO}_{2}$ flowrate, type of transport, transport distance and type of storage. Illustrative literature values are provided to support such preliminary estimates when detailed evaluations are not possible. It is worth noting that these considerations hold for CCS at both industrial processes and power plants.

- Technology maturity is an important factor in cost estimates, usually accounted for through the inclusion of process contingency costs for different levels of maturity and experience. 
Caution must be taken, however, when considering transferability of technology maturity from power sector applications to various industrial sectors. Impacts to consider include potential effects of CCS on product quality, plant maintenance, and operation of the CCS system under the specific conditions of the industrial facility.

Finally, we strongly recommend that future studies of industrial CCS applications make efforts to better document the adopted costing methodology, assumptions, and data sources, and to incorporate comprehensive uncertainty analyses and scenarios for key assumptions to increase the usefulness and robustness of cost estimates.

\section{Toward improved guidelines for uncertainty analysis of carbon capture and storage techno- economic studies}

This chapter reviews and provides guidance on available and emerging methods for uncertainty analysis in CCS techno-economic studies. It is intended to help accelerate continued methods development and their application to more robust and meaningful CCS performance and costing studies, as well as to provide an essential resource for all those developing, communicating, and using CCS costing studies.

We start by outlining the landscape of techno-economic modelling studies - from simplified to very rigorous and detailed, and studies that fall in between these extremes - and discusses the different purposes of uncertainty analysis. These include answering "what will" or "what if" kind of questions, model testing, or factor prioritization. It continues to describe existing uncertainty analysis methods: from local - ranging from 'one-at-a-time' to ' $\mathrm{N}$-ways sensitivity analysis' - to global, e.g., using Monte Carlo Simulation, and provides examples of these from the CCS literature. These methods are described following the "what, how, and when" structure, providing guidance on the use of such methods, and when they come to use and when better not.

The guidelines then review recently developed methods such as pedigree analysis, the pseudostatistical approach, or the use of surrogate models for global uncertainty analysis of integrated techno-economic models, when the technical models are too computationally heavy to run. These discussions lead to a guidance matrix and decision scheme for selecting uncertainty analysis methods and approaches for specific purposes, technology readiness levels (TRLs) and model types. These guidelines will also help the researchers and technology developers to critically analyse the techno-economic performance of the CCS technology under consideration.

Opportunities that can be achieved through advanced use of uncertainty analyses, such as design of experiments for CCS pilots or design of CCS chains under uncertainty are also discussed. Finally, we highlight the (un)availability of uncertainty analysis options in current flowsheeting software (such as Aspen Plus, gProms, HYSYS) and discusses other software that can be used for undertaking uncertainty analysis.

The key messages from the chapter can be summarized as follows:

- Proper use of uncertainty analysis in the performance of CCS techno-economic analyses can provide more robust understandings of technical and cost performance to modelling practitioners as well as policy- and decision-makers.

- The key to starting any uncertainty analysis is to first define its purpose thoroughly, and then to ensure that the most suitable type of uncertainty analysis for that purpose is selected. The 
choice also depends on the existing knowledge of the investigated technology and the associated techno-economic model and its inputs.

- Although most often so-called one-at-a-time sensitivity analysis is applied, the utility of this method is limited to techno-economic analysis, since most techno-economic analyses include non-linearities and parameter interactions. Therefore, a better practice is to use one-way or Nways sensitivity analysis if probability cannot be quantified in a credible way, and probabilistic uncertainty analysis if probability can be quantified. The latter is best suited to answer prognostic questions, but its utility depends on if credible probability density functions can be assigned to input parameters.

- Ideally, quantitative uncertainty analysis is complemented with qualitative uncertainty methods because they provide insights into the kinds of uncertainty that are unquantifiable, especially relevant to policy and decision making. Note that contrary to popular belief, much uncertainty resides in areas that are not quantifiable. This leaves parts of the total mass of uncertainties not investigated and communicated, providing an incomplete impression of the reliability, quality and accuracy of models and their results.

- Further expanding the capabilities of process simulation software to include advanced global uncertainty approaches would be very helpful, as most software, to our knowledge, do not (except gProms).

- There exists open-source, comprehensive, advanced uncertainty analysis toolboxes, but these require some skill in programming, perhaps providing a barrier for some techno-economic analysis practitioners. Therefore, further improvement of the user-friendliness of these toolboxes (e.g., by including graphic user interfaces) would aid in the wide adoption of advanced uncertainty analysis methods. 


\section{Table of contents}

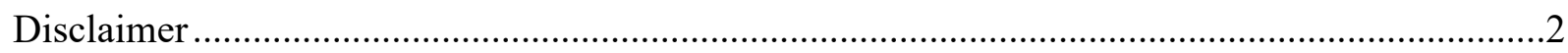

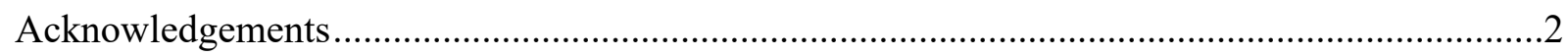

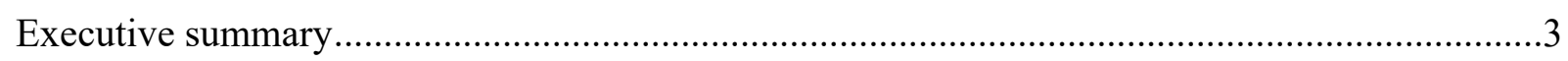

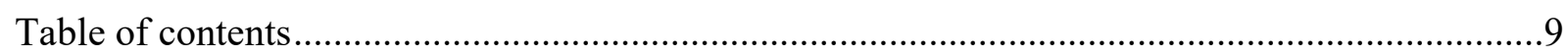

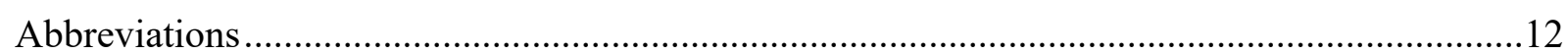

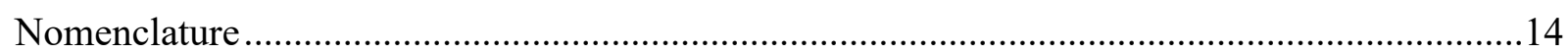

Chapter 1 : Towards improved cost guidelines for advanced low-carbon technologies..........15

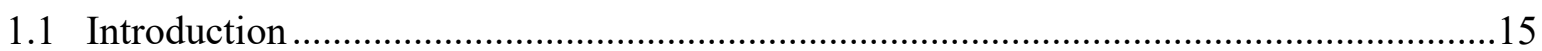

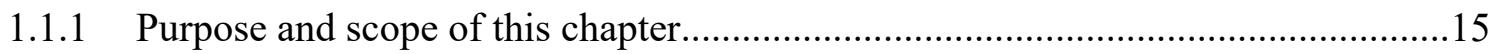

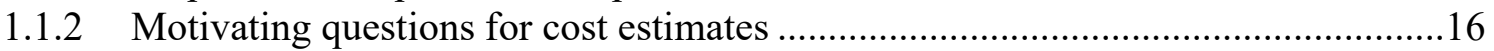

1.1.3 Different questions require different costing methods .........................................18

1.2 Overview of costing methods for advanced technologies.............................................18

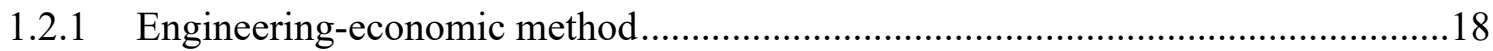

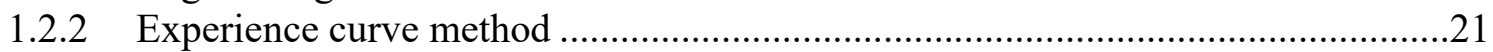

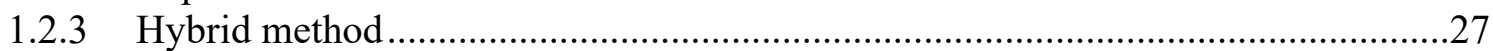

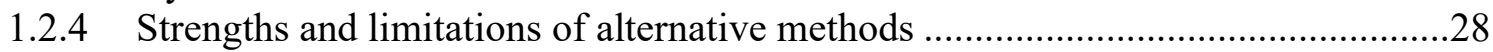

1.3 Guidelines for future cost estimates for Type 1 (What if) questions ................................30

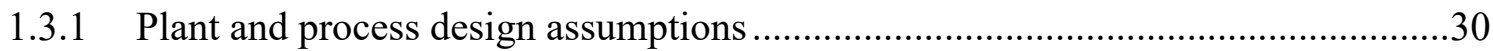

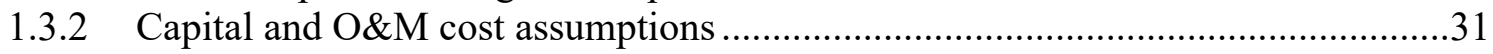

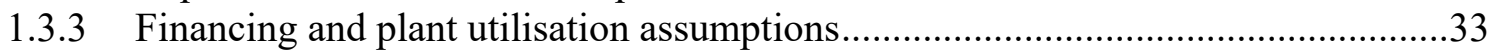

1.4 Guidelines for FOAK Cost for Type 2 (What will) questions .........................................35

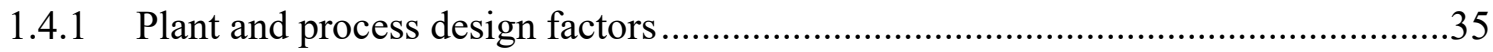

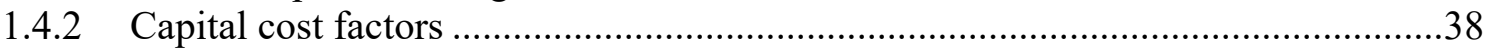

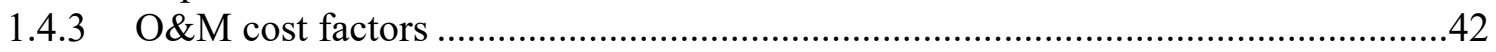

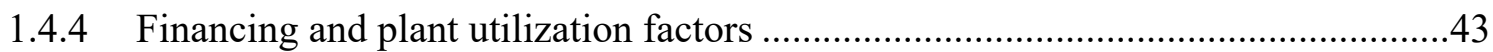

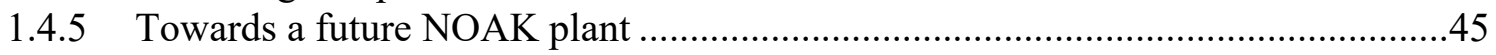

1.5 Guidelines for NOAK cost for Type 2 (What Will) questions .......................................45

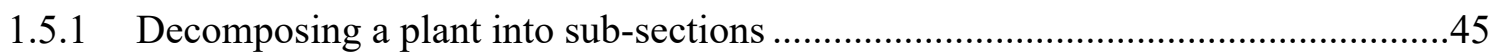

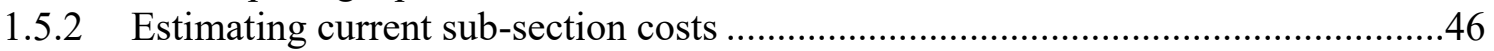

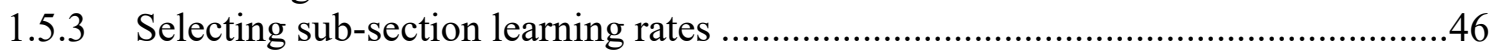

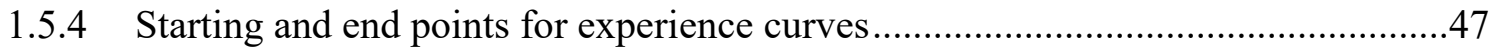

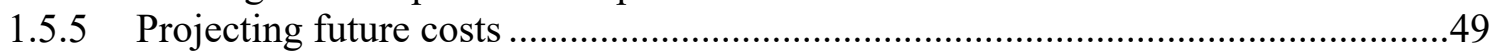

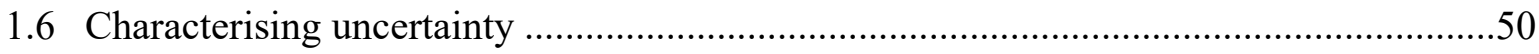

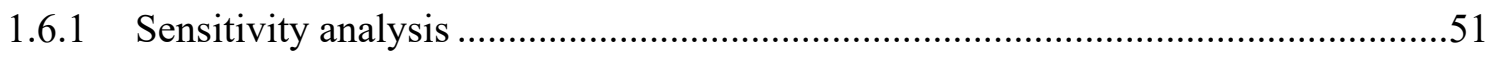

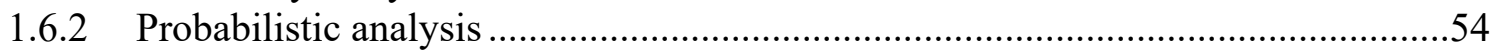




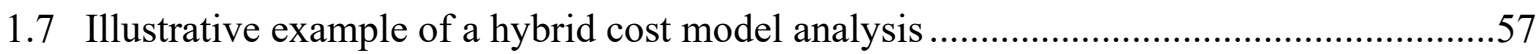

1.8 Discussion and conclusion

Chapter 2 : Towards improved cost evaluation of carbon capture and storage from industry 61

2.1 Introduction

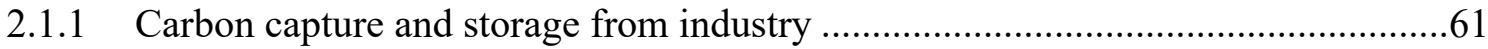

2.1.2 Towards improved cost estimates for CCS from industry ......................................62

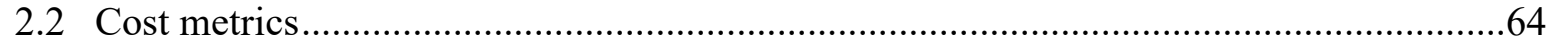

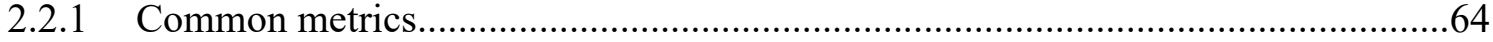

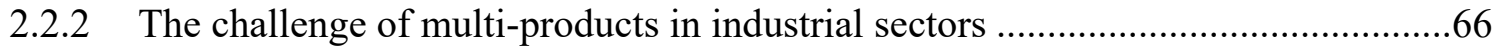

2.3 Considerations for improved assessment of key cost contributors .................................68

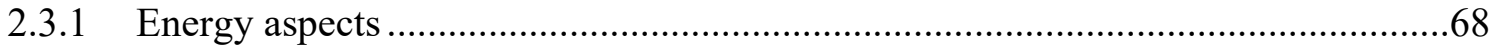

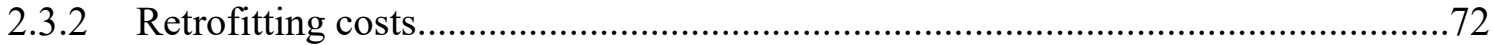

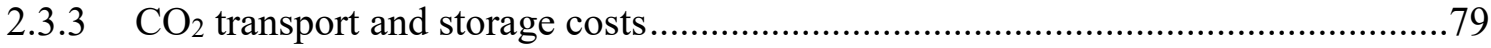

2.4 Transferability of experience and technology maturity from power to industry sectors ..83

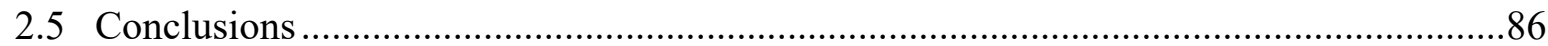

Chapter 3 : Toward improved guidelines for uncertainty analysis of carbon capture and

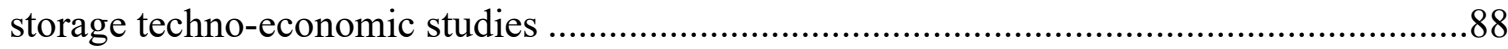

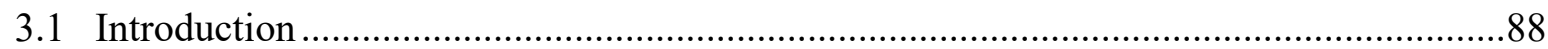

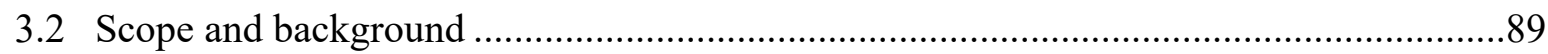

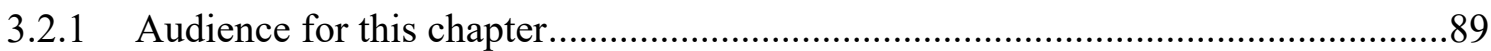

3.2.2 Types of techno-economic analyses and candidate parameter categories for

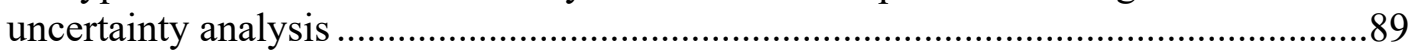

3.2.3 What do the existing TEA guidelines say about uncertainty assessment? ..............90

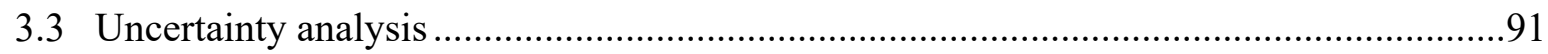

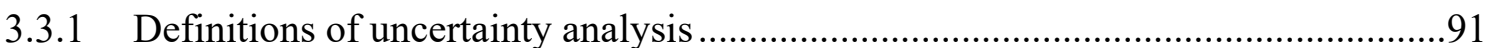

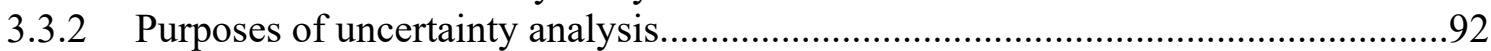

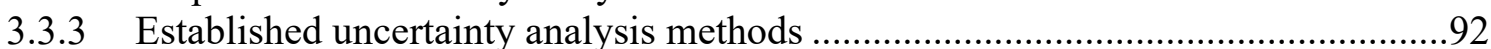

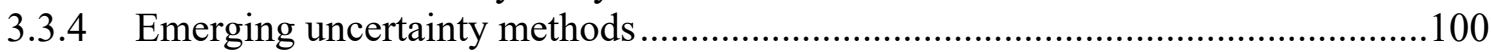

3.3.5 Strengths, weaknesses, and applicability of uncertainty methods to TEA .............108

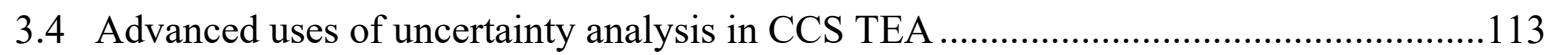

3.4.1 Using uncertainty analysis for design of experiments..........................................113

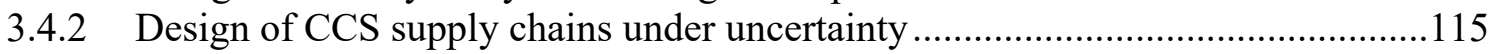

3.5 Available software for uncertainty and sensitivity analysis......................................117

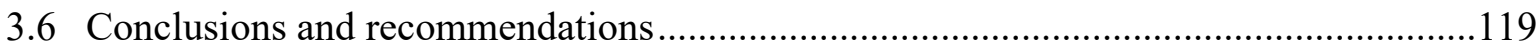

Appendix A: Screening questions for FOAK cost estimates..................................................121 
Appendix B: Location cost factors and other contingency cost guidelines for large-scale energy projects

Appendix C: Example assumptions and results for cost estimates using experience curves ......126

Appendix D: Example uncertainty in power plant cost using different analysis methods ..........130

Appendix E: EIA NEMS model assumptions for power plant learning rates

Appendix F: Overview of openly-available, highly transparent, and detailed techno-economic

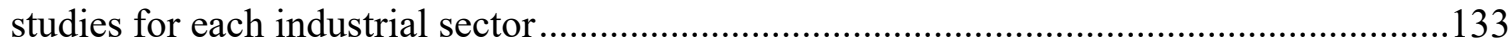

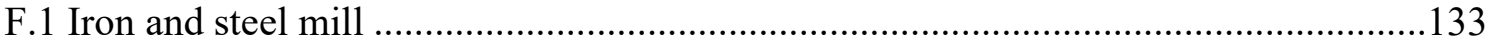

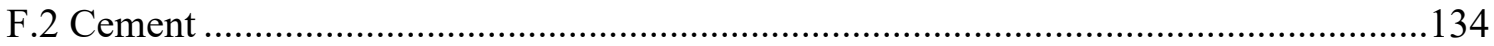

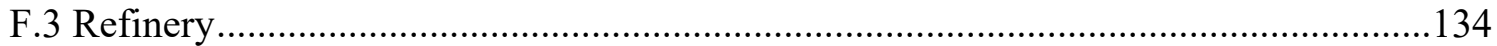

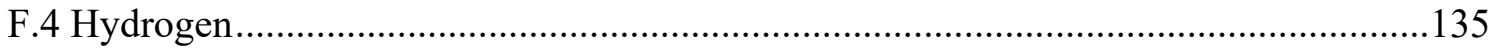

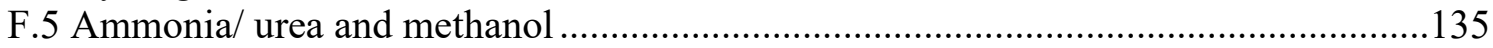

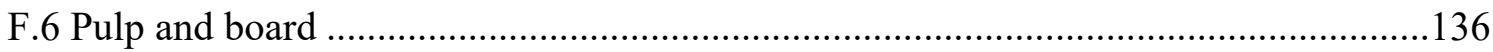

Appendix G: Other methods for calculating $\mathrm{CO}_{2}$ avoidance cost and their associated assumptions

Appendix H: Definitions of Technology Readiness Levels ..................................................138

Appendix I: Mathematical representations of uncertainty analysis ..........................................139

Appendix J: Guideline for the characterisation of probability density functions by Hawer et al.

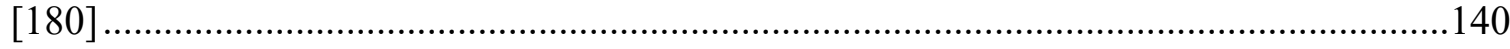

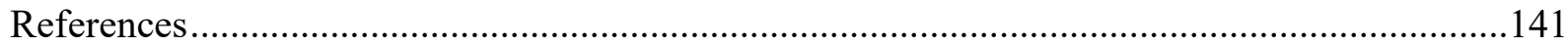




\section{Abbreviations}

\begin{tabular}{|c|c|c|c|}
\hline $\mathrm{AACE}$ & $\begin{array}{l}\text { Association for the Advancement } \\
\text { of Cost Engineering }\end{array}$ & $\begin{array}{l}\text { GJ } \\
\text { GTCC }\end{array}$ & $\begin{array}{l}\text { Gigajoule } \\
\text { Gas turbine combined cycle }\end{array}$ \\
\hline ADT & Air-dried ton & GW, GWe & Gigawatt electric \\
\hline ANN & Artificial neural network & GWh & Gigawatt-hour \\
\hline $\mathrm{AP}$ & Air pollution & $\mathrm{GWh}_{\text {cap }}$ & Gigawatt-hour installed capacity \\
\hline $\mathrm{B} \& \mathrm{~V}$ & Black \& Veatch & $\mathrm{H}_{2}$ & Hydrogen \\
\hline $\mathrm{BEC}$ & Bare erected cost & $\mathrm{HEV}$ & Hybrid electric vehicle \\
\hline BEV & Battery electric vehicle & HHV & Higher heating value \\
\hline BOP & Balance of plant & $\mathrm{HRC}$ & Hot-rolled coil \\
\hline BOS & Balance of system & IEA & International energy agency \\
\hline BP & By-product & IEAGHG & International Energy Agency \\
\hline BSP & Bleached softwood pulp & & Greenhouse Gas R\&D \\
\hline Btu & British thermal unit & & Programme \\
\hline CAC & $\mathrm{CO}_{2}$ avoidance cost & IECM & Integrated Environmental Control \\
\hline CAPEX & Capital expenditure & & Model \\
\hline $\mathrm{CCS}$ & $\mathrm{CO}_{2}$ capture and storage & IGCC & Integrated gasification combined \\
\hline $\mathrm{CDF}$ & Cumulative distribution function & & cycle \\
\hline $\mathrm{CF}$ & Capacity factor & IGFC & Integrated coal gasification fuel cell \\
\hline CHP & Combined heat and power plant & $\mathrm{kg}$ & Kilogram \\
\hline $\mathrm{CO}_{2}$ & Carbon dioxide & $\mathrm{kJ}$ & Kilojoule \\
\hline $\mathrm{COE}$ & Cost of electricity & $\mathrm{kPa}$ & Kilopascal \\
\hline CSP & Concentrated solar power & KPI & Key performance indicator \\
\hline DeSOx & Desulphurisation & $\mathrm{kW}$ & Kilowatt \\
\hline DOE & Department of Energy & $\mathrm{kWh}$ & Kilowatt-hour \\
\hline EBTF & European benchmarking taskforce & $\mathrm{LCOE}$ & Levelised cost of electricity \\
\hline EDDiCCUT & $\begin{array}{l}\text { Environmental due diligence of } \\
\mathrm{CO}_{2} \text { capture and utilisation } \\
\text { technologies }\end{array}$ & $\begin{array}{l}\text { LCOP } \\
\text { LCOKM } \\
\text { LH }\end{array}$ & $\begin{array}{l}\text { Levelised cost of product } \\
\text { Levelised cost of key material } \\
\text { Latin hypercube }\end{array}$ \\
\hline EIA & Energy Information Administration & LK & Lime kiln \\
\hline EPC & $\begin{array}{l}\text { Engineering, procurement, and } \\
\text { construction }\end{array}$ & $\begin{array}{l}\text { LNG } \\
\text { LR }\end{array}$ & $\begin{array}{l}\text { Liquefied natural gas } \\
\text { Learning rate }\end{array}$ \\
\hline EPRI & Electric Power Research Institute & $\mathrm{m}^{2}$ & Square meter \\
\hline ESP & Electrostatic precipitator & $\mathrm{m}^{3}$ & Cubic meter \\
\hline FCEV & Fuel cell electric vehicle & MARS & Multivariate adaptive regression \\
\hline FCF & Fixed charge factor & & splines \\
\hline $\mathrm{FF}$ & Factor fixing, or Fractional factorial & $\mathrm{MCMC}$ & Monte Carlo Markov chain \\
\hline FGD & Flue gas desulphurisation & MCS & Monte Carlo simulation \\
\hline FOAK & First-of-a-kind & MEA & Monoethanolamine \\
\hline FP & Factor prioritisation & MFB & Multi-fuel boiler \\
\hline $\mathrm{ft}^{3}$ & Cubic feet & MP & Main product \\
\hline GEP & General Electric Power & $\mathrm{MPa}$ & Megapascal \\
\hline GHG & Greenhouse gas & $\mathrm{Mt}$ & Million metric tons \\
\hline
\end{tabular}




\begin{tabular}{|c|c|c|c|}
\hline MW & Megawatt & TEA & Technoeconomic analysis \\
\hline MWh & Megawatt-hour & TOC & Total overnight cost \\
\hline NAT & $\mathrm{N}$-at-a-time (sensitivity analysis) & $\mathrm{t}$, tonne & Metric ton \\
\hline NEMS & National Energy Modeling System & TPC & Total plant cost \\
\hline NETL & $\begin{array}{l}\text { National Energy Technology } \\
\text { Laboratory }\end{array}$ & $\begin{array}{l}\text { TRL } \\
\text { TS\&M }\end{array}$ & $\begin{array}{l}\text { Technology Readiness Level } \\
\text { Transport, storage, and monitoring }\end{array}$ \\
\hline NCC & National carbon capture center & U.S. & United States \\
\hline NGCC & Natural gas combined cycle & UKM & Unit of key material \\
\hline NGO & Non-governmental organisation & UL & Underwriter's Laboratory \\
\hline NOAK & $\mathrm{N}^{\text {th }}$-of-a-kind & UQ & Uncertainty quantification \\
\hline NPV & Net present value & UV & Uncertain variable \\
\hline $\mathrm{O} \& \mathrm{M}$ & Operating and maintenance & WGS & Water gas shift \\
\hline OAT & One-at-a-time (sensitivity analysis) & wt $\%$ & Weight percent \\
\hline OPEX & Operational expenditure & $\mathrm{y}, \mathrm{yr}$ & Year \\
\hline$P$ & Pressure & ZEP & Zero Emissions Platform \\
\hline $\mathrm{PC}$ & Pulverized coal & $€$ & Euro \\
\hline PCE & Polynomial chaos expansion & $\$ / \mathrm{kW}$ & Dollars per kilowatt \\
\hline PDF & Probability distribution function & $\$ / \mathrm{kWh}$ & Dollars per kilowatt-hour \\
\hline PEFC & Polymer electrolyte fuel cell & $\$ / M W h$ & Dollars per megawatt-hour \\
\hline $\mathrm{PFC}$ & Process facilities cost & ${ }^{\circ} \mathrm{K}$ & Degrees Kelvin \\
\hline PR & Progress ratio & & \\
\hline PSA & Pressure swing adsorption & & \\
\hline PSUADE & $\begin{array}{l}\text { Problem solving environment for } \\
\text { uncertainty analysis and design } \\
\text { exploration }\end{array}$ & & \\
\hline PV & Photovoltaic & & \\
\hline QGESS & $\begin{array}{l}\text { Quality Guidelines for Energy } \\
\text { System Studies }\end{array}$ & & \\
\hline$R \& D$ & Research and development & & \\
\hline RD\&D & $\begin{array}{l}\text { Research, development, and } \\
\text { demonstration }\end{array}$ & & \\
\hline REC & Recovery boiler & & \\
\hline ROM & Reduced order model & & \\
\hline$R \& D$ & Research and development & & \\
\hline SA & Sensitivity analysis, or System area & & \\
\hline SCPC & Supercritical pulverized coal & & \\
\hline SCR & Selective catalytic reduction & & \\
\hline SDoE & Sequential design of experiments & & \\
\hline SMR & Steam methane reforming & & \\
\hline $\mathrm{SO}_{2}$ & Sulphur dioxide & & \\
\hline SOFC & Solid oxide fuel cell & & \\
\hline SEWGS & Sorption-enhanced water-gas shift & & \\
\hline SRL & System readiness level & & \\
\hline $\mathrm{T}$ & Temperature & & \\
\hline $\mathrm{T} \& \mathrm{~S}$ & Transport and storage & & \\
\hline TASC & Total as-spent cost & & \\
\hline TCM & Technology centre Mongstad & & \\
\hline $\mathrm{tCO}_{2}$ & Tonne $\mathrm{CO}_{2}$ & & \\
\hline
\end{tabular}




\section{Nomenclature}

$\alpha \quad$ Specific cost at unit cumulative capacity and unit knowledge stock

$a \quad$ Capital cost per unit for the first (or initial) unit of capacity (Chapter 1)

a Amount of sampled points in addition to its extremes and the base case (Chapter 3)

$b \quad$ Learning rate exponent

$b_{l b d} \quad$ "Learning-by-doing" parameter

$b_{l b r} \quad$ "Learning-by-researching" parameter

C Computational cost

E $\quad$ Expected value

$\hat{g} \quad$ Reduced order model

$I_{\mathrm{CCS}, \mathrm{a}} \quad$ Annualised investment cost of CCS implementation

$k \quad$ Number of varied input parameters

$(L C O K M)_{c c s}$ Levelised cost of the key material(s) of the industrial plant with CCS

$(L C O K M)_{\text {ref }}$ Levelised cost of the key material(s) of the industrial plant without CCS

LR Learning rate

$m \quad$ Model output

$n \quad$ Model input

$\dot{M}_{\mathrm{CO} \text {,avoided }}$ Annual reduction in $\mathrm{CO}_{2}$ emissions due to $\mathrm{CCS}$ for a plant producing the same amount of product(s) with and without CCS

$\dot{M}_{\mathrm{CO}, \text { avoided,i }} \quad$ Mass of $\mathrm{CO}_{2}$ avoided by CCS implementation in year i.

$N P V_{\text {CCS }} \quad$ Annual operating cost of the CCS facility

$O_{\mathrm{CCS}} \quad$ Net present value of total annual CCS costs (which may vary from year to year)

$\mathbb{P} \quad$ Probability

$p_{i} \quad$ Preference for choice i

PR Progress ratio

$\left(t_{C O 2} / U_{K m}\right)_{C C S}$ Mass amount of $\mathrm{CO}_{2}$ emitted per unit of key material(s) with CCS

$\left(t_{\mathrm{CO} 2} / U_{\mathrm{Km}}\right)_{\text {ref }}$ Mass amount of $\mathrm{CO}_{2}$ emitted per unit of key material(s) without CCS

$U \quad$ Uniform distribution

$\mathbf{v} \quad$ Vector of deterministic input parameters

$x \quad$ Ratio of cumulative to initial capacity of the technology

$y \quad$ Capital cost per unit for the $x^{\text {th }}$ unit of plant capacity

$\boldsymbol{\theta} \quad$ Set of stochastic input parameters 


\section{Chapter 1 : Towards improved cost guidelines for advanced low-carbon technologies ${ }^{1}$}

\subsection{Introduction}

Research and development (R\&D) programs on energy technologies and other industrial processes seek improved, lower-cost systems for producing electricity, fuels, chemicals, and other desired products that reduce environmental emissions of greenhouse gases and other pollutants. Lower-cost technologies for carbon capture and storage (CCS), as well as cleaner, more efficient industrial processes, power plants, and other energy conversion systems, are among the advanced technologies being pursued by researchers worldwide. This chapter is aimed at the wide variety of audiences and stakeholders in the public and private sectors who generate or use cost estimates for such technologies to make or inform decisions regarding R\&D programs, investments, policy options, or communications about the outlook for advanced CCS and other low-carbon technologies.

\subsubsection{Purpose and scope of this chapter}

To assess the viability and competitiveness of a new technology, a common figure of merit is the future cost of a commercial-scale installation once the technology is mature and widely deployed. This cost is commonly referred to as the " $N^{\text {th }}$-of-a-kind" (NOAK) plant cost. Such costs may be sought for a single technology (such as an advanced carbon capture process) as well as for an integrated plant or system employing one or more advanced technology components or novel process integration schemes. At present, however, methods and assumptions for estimating NOAK costs vary widely, resulting in reported NOAK costs that are often ambiguous (at best) or misleading (at worst). This chapter seeks to improve the clarity, consistency, and utility of NOAK cost estimates for advanced technologies by first discussing the purposes of such estimates and then the methods appropriate for different objectives. The guidance in this chapter is directed mainly at preliminary cost studies conducted in the early stages of a potential project for purposes of scoping and initial feasibility assessments (in contrast to the far more detailed and costly Front-End Engineering and Design studies used to support final investment decisions). While originally motivated by a focus on advanced carbon capture processes, the methodological issues and approaches to cost estimation discussed in this chapter also apply to a broader array of low-carbon technologies of interest for power generation and other industrial processes.

Throughout this chapter, the term "advanced" refers to any technology or concept that is still in an early (pre-commercial) stage of development, including technologies that are sometimes referred to as novel, emerging, step-out, breakthrough, game-changing, leapfrog, transformational, nextgeneration, or other similar terms. On the nine-point Technology Readiness Level (TRL) scale [7] the technologies of interest in this chapter primarily encompass TRL values from about 3 to 7, which is the major focus of current R\&D programs. ${ }^{2}$

The remainder of this section discusses the objectives and questions that motivate a cost analysis of an advanced technology. Clarity of objectives is shown to be essential for choosing an appropriate costing

\footnotetext{
${ }^{1}$ This chapter was authored by Edward S. Rubin, Niels Berghout, George Booras, Tim Fout, Monica Garcia, Shareq Mohd Nazir, Andrea Ramirez, Simon Roussanaly, and Mijndert Van der Spek. It builds on the paper, "Rubin, Improving cost estimates for advanced low-carbon power plants," International Journal of Greenhouse Gas Control 88, 1-9. 2019" available at https://doi.org/10.1016/j.ijggc.2019.05.019. The corresponding author for this chapter is Edward S. Rubin (e-mail address: rubin@,cmu.edu).

${ }^{2}$ The methods described in this chapter also would apply to the smaller number of advanced technologies that reach TRL 8 or 9. For conceptual designs at TRLs 1 or 2 a detailed cost evaluation is generally not recommended (e.g., see [8])
} 
method. Section 1.2 then presents an overview of the two primary costing methods used for energy technologies: the engineering-economic ("bottom-up") method and the experience curve method. A hybrid method combining these two approaches is introduced as an improved approach to forecast the NOAK costs of advanced technologies for purposes of preliminary assessments.

Sections 1.3 and 1.4 discuss in more detail the use of the engineering-economic method to address the two types of questions that commonly motivate a cost analysis. Here, a distinction is made between the use of a bottom-up costing method for mature technologies (Section 1.3) versus its use for projects employing advanced technology components (Section 1.4). Section 1.5 then describes the use of the hybrid approach to estimate the expected NOAK cost of an advanced technology. Section 1.6 highlights the importance and methods of uncertainty analyses for all cost estimates. Section 1.7 presents an illustrative application of the hybrid costing method. Section 1.8 then concludes with a summary discussion and conclusions.

\subsubsection{Defining objectives for advanced technology cost estimates}

To help identify the most promising advanced technology options, techno-economic studies are regularly carried out by researchers and technology developers in industry, government, and academia. Individuals and organisations also regularly publish cost estimates for advanced technologies. In general, the objective of such cost estimates is to assess the economic viability or competitiveness of an advanced technology relative to an existing current technology. In some cases, comparisons with other advanced technologies also are sought. Such estimates are used by technology developers and researchers to quantify the potential benefits of a new technology relative to a current or competing technology, or a cost reduction goal.

In most cases, advanced technology cost estimates represent the NOAK cost that could be achieved with sufficient replication and maturity if all design goals are met. Towards that end, the most common costing method is a detailed bottom-up engineering-economic approach in which plant and process designs are first specified together with appropriate performance and operating parameters needed to determine all mass and energy flows for the specified system. Based on this information, the cost of purchasing and installing all plant equipment is then estimated for a particular geographic location. This cost is then aggregated with other capital cost elements to obtain the total capital required to construct the overall process or plant (which is often assumed to be at a generic location in a given country). This capital cost is then combined with estimates of annual operating and maintenance (O\&M) costs to obtain the total annualized cost of the facility and the cost per unit of product (e.g., per megawatt-hour of electricity in the case of a power plant) for specified financing terms and plant lifetime (for details of these cost calculations and related nomenclature see e.g., [6]).

A similar bottom-up cost analysis is then used to calculate the performance and cost of a reference plant or process, typically a plant employing current (baseline) technology. Differences between the reference plant and the advanced technology plant are used to quantify the economic and performance benefits of the advanced technology. In many cases, the advanced plant design is identical to the reference plant except for a new technology, such as a novel system for post-combustion $\mathrm{CO}_{2}$ capture and storage.

While the approach outlined above may at first glance appear appropriate for evaluating the future (NOAK) economic competitiveness of an advanced technology, a closer look reveals a methodological flaw for analyses seeking to estimate the likely future cost of a new technology. This can be seen by examining in more detail the types of questions that motivate advanced technology cost estimates.

\subsubsection{Motivating questions for cost estimates}

To evaluate appropriate costing methods for advanced technologies it is important to first carefully frame the questions that motivate the cost analysis. A review of recent studies [9] indicates that most questions fall into two general categories, referred to in this chapter as Type 1 ("What If") and Type 2 ("What Will") questions. 


\subsubsection{Type 1 questions: What If?}

This category of questions arises largely from the R\&D community. This group is concerned with issues such as setting targets for R\&D programs, identifying the best options to meet technology cost and/or performance goals, and quantifying the expected cost reductions of a successful R\&D effort. Accordingly, this group asks questions such as "What R\&D goals are needed to achieve a desired cost target for a particular technology or advanced power system?" Conversely, "What would be the cost (or cost reduction) of an advanced technology if it successfully achieves specified R\&D goals?"

This class of questions - which includes a number of variants - characterizes the objective of most cost studies carried out by DOE/NETL and others for advanced energy and environmental technologies. Such studies assume a commercial plant design that meets specified R\&D performance goals and component cost targets for the advanced process or system, such as a novel carbon capture process or an advanced power plant design employing several advanced components (e.g. [10-12]). In some cases, R\&D goals and targets may reflect extrapolations from bench-scale data or the use of commercial analogies to a novel technology.

In all cases, however, a what-if analysis is a hypothetical construct for a specified process design and a specified set of performance and cost assumptions - typically for an integrated plant with one or more unique (advanced) technology components. Based on these assumptions, a bottom-up engineeringeconomic analysis is commonly employed to calculate its cost —often referred to as the "NOAK cost."

However, a bottom-up cost estimate of this kind does not represent the actual expected cost of the technology at some future time since one does not know whether or when the assumed cost and performance targets can actually be achieved (either in general, or at a specific location). Rather, it is an estimate of the potential cost of an advanced technology for the optimistic case in which all R\&D goals and assumptions are realized at some point in the future. Thus, it is better described as an "aspirational NOAK cost" or "NOAK cost goal." Note that generally there is no specific timeframe inherent in this type of analysis.

Two common variants of the "What If" questions above are to ask, "What would it take for an advanced technology or system to be competitive with a current baseline system?" or, "How much cheaper would Technology X be compared to Technology Y if its performance and cost goals are met?" In this approach, the performance and cost of all conventional plant components are first established using baseline data, leaving a cost gap or target that the advanced technology must meet in order to be competitive. This approach to costing is discussed in more detail by Guandalini, et al. [13]. In other cases, a scenario analysis or a simple sensitivity analysis is used to identify the conditions (parameter values) under which an advanced technology becomes attractive. Professional judgments also may be applied to assess whether such conditions appear feasible or plausible for these hypothetical outcomes.

\subsubsection{Type 2 questions: What Will?}

A second category of questions is motivated largely by the investment, user, and energy modelling communities (including policy analysts). These groups are interested in the future cost of new technologies at given points in time, and in the cost-competitiveness of an advanced technology relative to existing systems or other competing options. Questions here include: "What will be the likely cost of advanced Technology $X$ in future year $Y$ ? When will the cost of Technology $\mathrm{X}$ fall below some specified level (or below the cost of a competing technology)?" and, "What will be the NOAK cost of Technology $X$ ?" This can be described as the "expected NOAK cost" or "projected NOAK cost."

Current methods to address this class of "What Will" question also commonly employ a bottom-up (engineering-economic) analysis of a proposed process and plant design that incorporates the advanced technology of interest. The resulting cost estimate also is typically reported simply as the NOAK cost of the advanced system. However, many cost studies fail to clearly say whether the reported NOAK cost is intended to represent the actual expected cost of a mature plant (i.e., the answer to a what-will question), or whether it represents only a hypothetical (or aspirational) what-if case. Absent that 
distinction, the implication left in many studies is that the NOAK cost based on a bottom-up estimate indeed represents an expected future cost. In other cases, the meaning of an NOAK cost remains ambiguous.

Later in this chapter, in Section 1.8, we recommend adoption of the nomenclature highlighted above to distinguish between NOAK cost estimates motivated by the two types of questions.

\subsubsection{Different questions require different costing methods}

The key flaw with the common bottom-up approach to what-will cost estimates is that an engineeringeconomic cost analysis is simply not appropriate or intended for estimating the actual future NOAK cost of an advanced technology that is not yet commercial. By definition, the only way to know the true NOAK plant cost is by building $N$ plants. Similarly, the only way to reliably estimate the cost of the $N^{\text {th }}$ plant using a bottom-up analysis is by first having built and operated many plants (ideally, $N-1$ ) of a similar design.

Thus, one cannot correctly claim or suggest that a bottom-up cost estimate for a technology that has never been built and operated at scale represents the true future design and cost of that system, were it to be deployed many $(N)$ times at a commercial scale. Rather, experience shows that technology designs and process flowsheets evolve and change over time as successful technologies mature and become more widely deployed - a process that typically takes several decades or more for large-scale energy technologies. In that dynamic, the capital and operating costs of new system components also change considerably over time, as detailed case studies have shown (e.g., [14]). Thus, one cannot confidently know today what the design and other attributes of a future successful technology will be. One can only hypothesize about future designs, which is inherently a what-if rather than a what-will type of analysis.

For this reason, different costing methods for advanced technologies are needed to address the two types of questions posed above. The following section presents an overview of the methods applicable to each type of question. Subsequent sections of this chapter then present guidelines for the application of these methods.

\subsection{Overview of costing methods for advanced technologies}

Three costing methods are summarized in this section: the engineering-economic method, the experience curve method, and the hybrid method that combines the first two. Further details on the use of each method are presented in subsequent sections of this report.

\subsubsection{Engineering-economic method}

This is the detailed bottom-up method commonly used to calculate the current cost of a project employing technology that is commercially available, often from multiple vendors. It is the principal method used by the Electric Power Research Institute (EPRI), , the U.S. Department of Energy's National Energy Technology Laboratory (NETL) and others for technology cost estimates. Details of the method as applied to power plants were described in an earlier white paper prepared by an international Working Group [15]. That same general framework applies equally well to other industrial processes, with adjustments for unique items and nomenclature in a given industry.

While that effort advanced the systematic use of relevant cost categories by major R\&D organisations and firms, some differences are still found in cost-related nomenclature across different organisations. The current chapter often references the terms used by NETL, whose publically available technical reports and cost methodology guidelines are widely used and cited by others. Those guidelines for engineeringeconomic cost estimates are described most recently in the 2019 report, "Cost Estimation Methodology for NETL Assessments of Power Plant Performance" [16]. They are based in large part on the costing methodology first developed by AACE International (formerly known as the Association for the Advancement of Cost Engineering), which established five classifications for cost estimates based on the 
level of project definition and detail, with increasing levels of accuracy for the resulting cost estimates [17]. This also the basis for guidelines subsequently adopted by EPRI [18, 19]. A serie of additional reports provides further guidance on specific aspects and assumptions used in NETL studies [20].

Based on specified plant and process designs, the required energy and mass flow are calculated and used to size all major equipment and plant components. The total capital requirement is then calculated as shown in Figure 1-1, which illustrates how various components of capital cost are aggregated to yield the total as-spent cost (TASC) for a particular system. While many recent studies are for fossil-fuelled power plants, the cost categories in Figure 1-1 are generic and can be applied to any technology of interest. The cost methodology also defines in detail the procedure used to combine the capital cost with financial parameters and annual O\&M costs to obtain the overall cost of electricity (COE) generation for a power plant in a given year. More commonly, annual O\&M costs are assumed to be constant for all years of operation when calculating the levelised cost of electricity (LCOE) over the life of the plant.

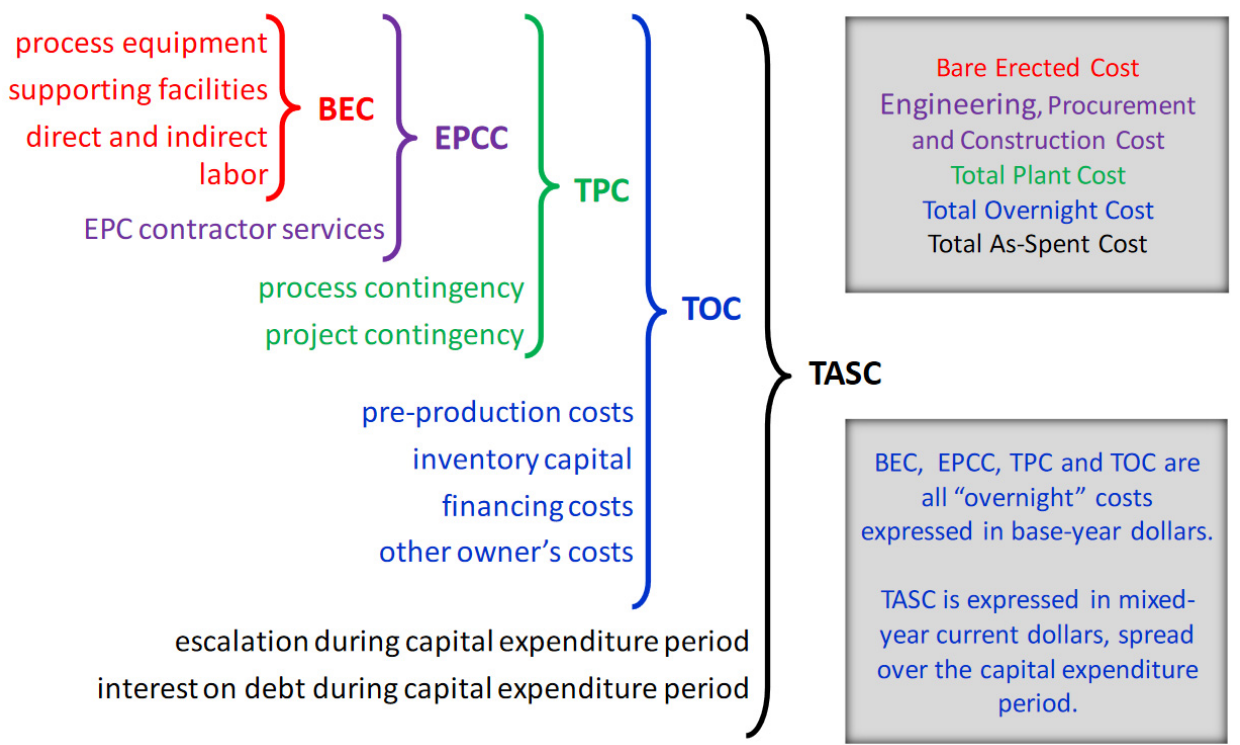

Figure 1-1. Schematic of the NETL engineering-economic method for capital cost estimates [16]

The basic building block of the engineering-economic cost estimate is an equipment list based on a specified process design and flowsheet that is intended to achieve a specified level of plant performance (e.g., output, efficiency, emissions, availability) over its lifetime. As indicated in Figure 1-1, the total cost of purchasing and installing all process or system components is generally referred to as the bare erected cost (BEC) [16]. Other capital cost elements in Figure 1-1 are typically estimated as a percentage of this amount (which some cost methods refer to as the total direct cost or process facilities cost).

For current commercial technologies, the BEC is commonly based on vendor quotes and/or recently published costs for major plant components. In some studies, such estimates are developed by a contractor providing engineering, procurement, and construction (EPC) services. Where such data are not readily available, equipment costs may be estimated using engineering handbooks, computer modelling software, or other methods $[16,21]$. More complete descriptions of all cost items for power plant studies also can be found in Rubin, et al. [15]. Readers of this chapter who are not already familiar with the AACEEPRI-NETL cost study categories and methodology should review that earlier work, which is foundational to the current report, focused on advanced technology applications.

\subsubsection{Application to Type 1 (What If) questions}

For advanced technologies that are not yet commercial and have not yet been built and operated at scale, a detailed engineering-economic cost analysis is appropriate to address Type 1 (What If) questions that are based on an assumed plant or process design with assumed values of technology performance and/or 
cost parameters. By definition, such assumptions may or may not represent actual outcomes were the new technology to be built and widely deployed at scale. Typically, such analyses are carried out to assess the long-term potential and competitiveness of advanced technology components or novel plant designs and process integration schemes that meet specified performance and/or component cost goals. Such bottomup studies, however, must take care to recognize that cost results represent a hypothetical case, and not a projection of the likely expected cost at some future time, given where the technology is today.

DOE/NETL regularly employs engineering-economic cost estimates for what-if studies of advanced technologies employing fossil fuels. Figure 1-2 illustrates typical results from one such study. In this case the technology is a power plant employing an integrated coal gasification fuel cell (IGFC) system to achieve higher efficiency than a conventional coal-fired power plant [22]. The results, based on computer modelling studies, show the incremental effects on overall plant efficiency and cost of meeting R\&D goals for seven different parameters. This type of analysis asks what the overall plant cost would be if each technology component achieves specified goals for cost and performance. Typically, no specific timeframe is provided in this type of analysis.
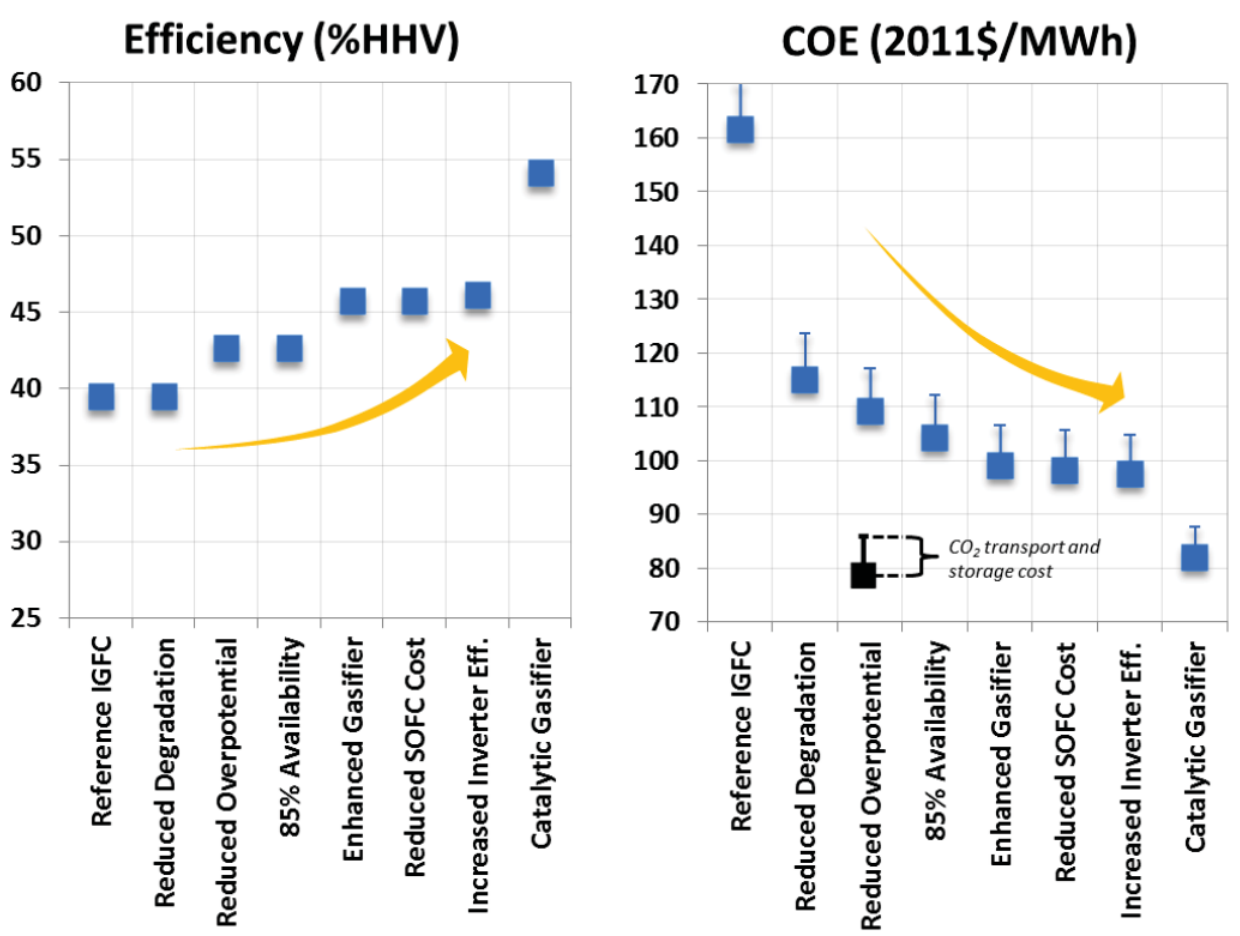

Figure 1-2. Cumulative impact on plant efficiency and cost of electricity (COE) of improvements in advanced IGFC technology [22]

Critical to analyses of this type is that the assumed values of certain performance and cost parameters be consistent with the premise of the analysis, e.g., an NOAK design for a mature technology. Section 1.3 of this chapter later elaborates on this topic.

\subsubsection{Application to Type 2 (What Will) questions}

As noted earlier, a detailed bottom-up cost analysis is not appropriate for an advanced technology if the objective is to estimate its actual cost at some future time when it has matured and been widely deployed. Rather, use of the engineering-economic cost estimate for what-will questions is appropriate only for a project to be built now or in the immediate future, based on current knowledge (and uncertainty) about the technology's performance and cost at scale. For an advanced technology that has not yet been built at scale, this near-term project would represent, by definition, the first-of-a-kind (FOAK) installation of a full-sized power plant or sub-system. 
Section 1.4 later presents a more detailed discussion of how the engineering-economic method can be applied to estimate the FOAK cost of an advanced technology. Once such an estimate is obtained, it is then used in conjunction with the experience curve costing method to forecast the expected future cost of the advanced technology. The following section presents a brief review of the experience curve method.

\subsubsection{Experience curve method}

Experience shows that cost estimates for early-stage technologies tend to be optimistic and significantly lower than the actual cost of technologies that advance from research, development, and demonstration (RD\&D) to commercial deployment at scale. Subsequently, the relatively high cost of early deployments tends to decline as the technology is adopted and matures, as depicted in Figure 1-3. This decline reflects a "learning rate" which can be estimated mathematically, thus providing an alternative costing method for advanced technologies once they reach the stage of large-scale deployment.

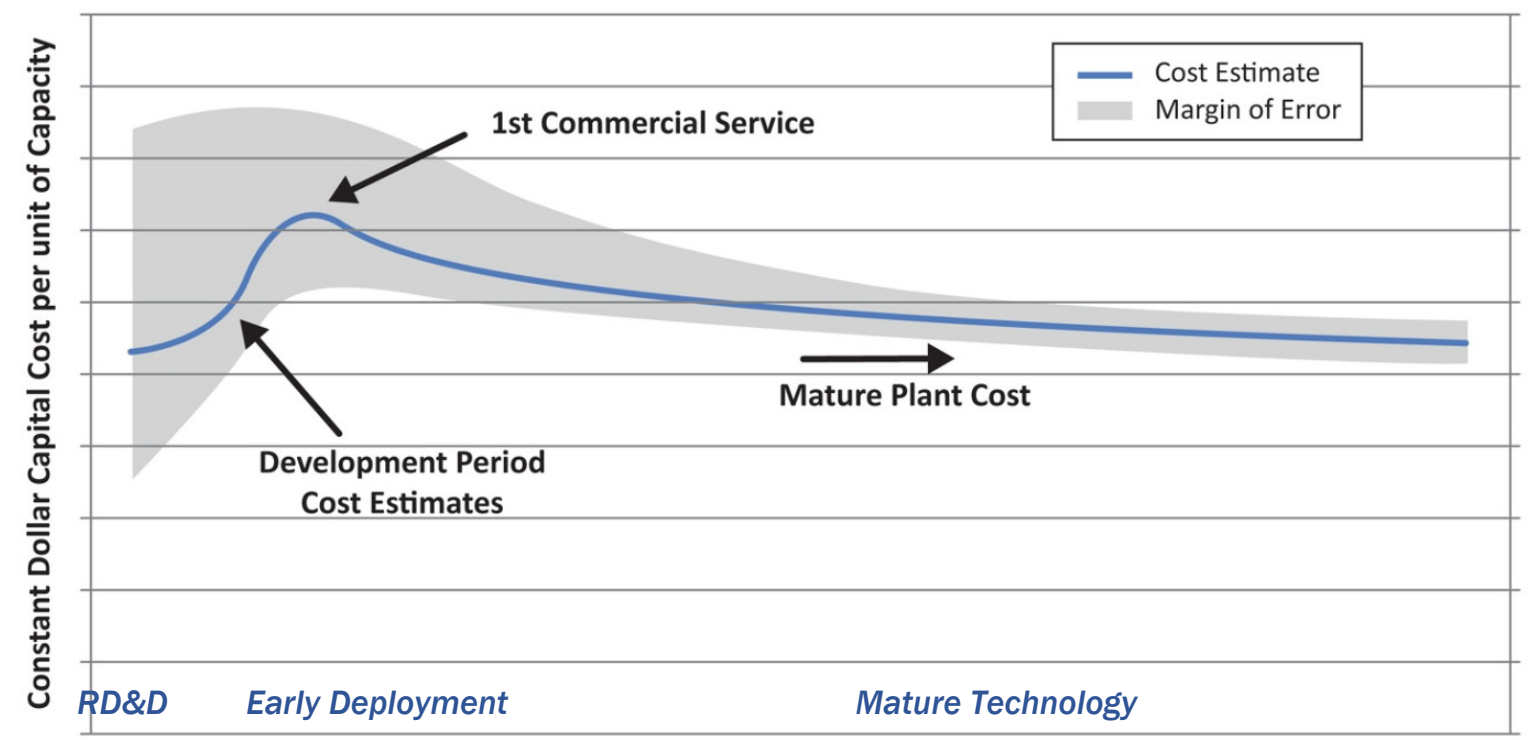

Stage of Technology Development

Figure 1-3. Typical cost trajectory of an advanced technology [23]

Figure footnote: the shaded area surrounding the curve reflects the varying level of accuracy associated with design estimates at different stages.

\subsubsection{Empirical and theoretical framework}

The use of experience curves - also commonly called "learning curves"-represents a top-down method of estimating the future cost of a technology based on historical trends for similar technologies. This method has its origin in the study of aircraft manufacturing in the 1930s, where it was found that the time required to construct a particular type of aircraft decreased in a predictable fashion as the task was repeated. Construction time versus cumulative production was found to be well-fit by a log-linear equation. Later, that equation was found to also fit observed trends in the capital cost of a variety of technologies as a function of cumulative production or installed capacity [24]. This experience curve equation is generally expressed as [25]:

$$
y=a x^{-b}
$$

where, for power plant capital cost:

$y=$ capital cost per unit for the $x^{\text {th }}$ unit of plant capacity

$a=$ capital cost per unit for the first (or initial) unit of capacity

$x=$ ratio of cumulative to initial capacity of the technology

$b$ learning rate exponent 
The empirical basis for such relationships typically reflects multiple generations of a technology deployed at plants of different sizes and designs. Cumulative installed capacity rather than number of installations is thus the most common independent variable used to develop experience curves. Some experience curve studies, however, use the cumulative value of energy production cost $(\$ / \mathrm{MWh})$ as the independent variable, $x$, rather than cumulative capacity. Experience curve models based on Equation 1 also have been derived for annual O\&M costs as well as for capital costs [26].

A defined parameter of learning curve models is the progress ratio, $P R$, defined as the fraction of the initial cost after a doubling of the initial capacity (i.e., a value of $x$ equal to two). Thus:

$$
P R=2^{-b}
$$

where, $b$ is the exponent in Equation 1 . A complementary parameter, widely used in the learning literature, is the learning rate, $L R$, defined as the fractional reduction in cost for a doubling of the initial capacity. Thus, the learning rate is defined mathematically as:

$$
L R=1-2^{-b}=1-P R
$$

In this chapter, learning rate is used as the preferred measure of cost reductions achieved through technological learning, reported either as a decimal value or as a percentage. Given an observed value of the learning rate, the numerical value of the exponent $b$ can then be calculated from Equation 1 and Equation 3 as:

$$
b=-\log (1-L R) / \log (2)
$$

Figure 1-4 illustrates graphically the exponential decline in cost for different learning rates as a function of the cumulative deployment of a technology based on an arbitrary unit cost of 1000 for the first unit of capacity. In this example, learning rates range from 1 percent to 30 percent cost reduction for each doubling of cumulative capacity. As shown later in Section 1.2.2.2, this range encompasses most of the empirical values found for energy-related technologies.

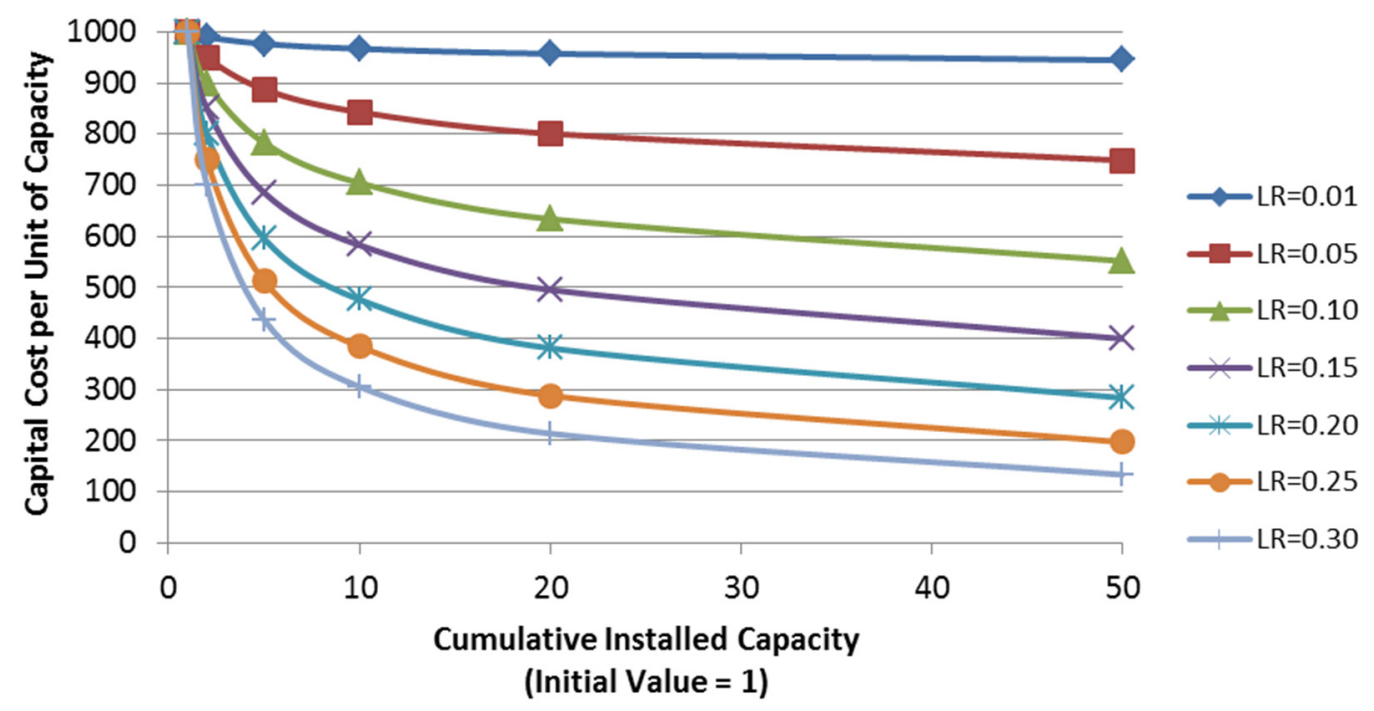

Figure 1-4. Illustrative cost trends for different learning rates

Equation 1 also can also be re-written as a linear equation by taking the logarithm of each term, yielding:

$$
\log y=\log a-b(\log x)
$$

This log-linear form is often used to display experience curve data. As illustrated in Figure 1-5, the result is a linear decline in cost with increasing cumulative capacity on a log-log scale. 


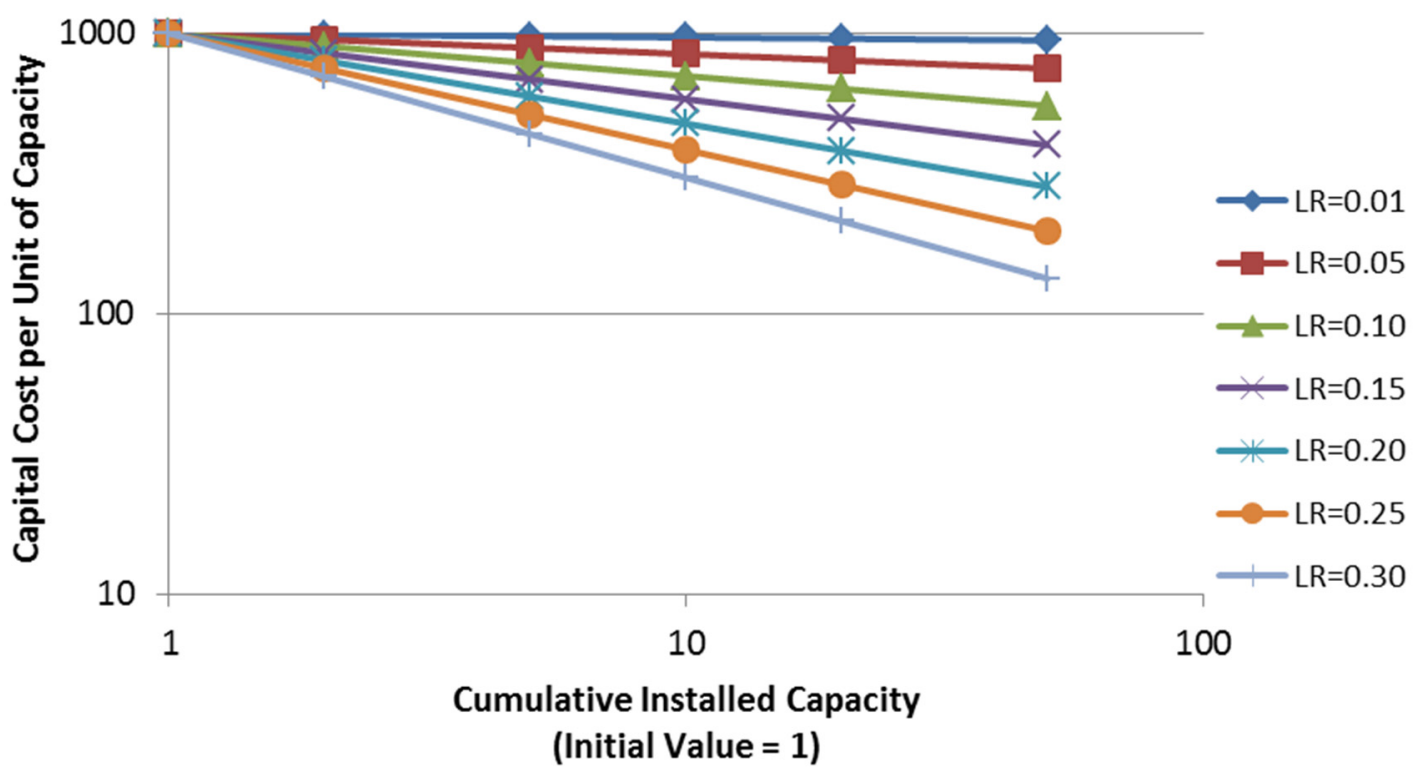

Figure 1-5. Illustrative cost trends for different learning rates on a log-log scale

Research on energy technologies also has sought more complex multi-factor model formulations to explain observed cost trends, especially the effect of R\&D spending (also referred to as the "knowledge stock") on technology cost. Thus, there are examples in the literature of experience curves of the form:

$$
\log y=\alpha+b_{l b d}(\log x)+b_{l b r}(\log R)
$$

where, $\alpha$ is the specific cost at unit cumulative capacity and unit knowledge stock, $b_{l b d}$ is the "learningby-doing" parameter, $b_{l b r}$ is the "learning-by-researching" parameter, $R$ is the cumulative $\mathrm{R} \& \mathrm{D}$ investment, and $y$ and $x$ are as defined earlier in Equation 1.

While multi-factor models of this type that distinguish learning-by-doing from other factors that influence an experience curve are conceptually appealing, data to support them quantitatively are typically sparse. Thus, a recent literature review showed that the one-factor experience curve given by Equation 1 is the most prevalent model across a wide range of studies and energy technologies, with only a few multi-factor models, primarily for wind turbine power plants [27].

\subsubsection{Learning rates for energy conversion technologies}

Studies of learning rates for energy-related technologies have included a wide variety of electric power generation technologies and other large-scale energy conversion systems, as well as small-scale devices like energy-efficient air conditioners and light bulbs [28]. Among these, the most heavily-studied technologies have been renewable energy systems utilising wind and solar energy. Relatively few studies have been reported of past learning rates for industrial processes.

Table 1-1 summarizes the one-factor learning rates found from studies of power generation and other energy-related technologies. The data come from several recent papers, each of which reviewed and summarized peer-reviewed journal studies of learning rates for energy-related technologies. All rates shown are for new facilities and may not apply to retrofit installations with unique site-specific cost elements. In addition to learning rates based on observed cost reductions in the past, several of the review papers also reported expected future learning rates for selected technologies based on modelling studies or other estimates. Several of those "prospective" learning rates also are included in Table 1-1 and are designated by italics to distinguish them from historical rates. Another recent review paper [29] also reports learning rates from both retrospective and prospective studies, but does not present separate summaries of those categories. The reported learning rates again are predominantly from wind and solar PV systems, with values similar to those in Table 1-1. 
One general observation from these data is that modular/standardized technologies that are produced in large quantities (such as solar PV modules) are typically more conducive to faster technological learning and cost reductions than more complex technologies that are tailored to specific circumstances and produced in smaller quantities (such as nuclear power plants).

Table 1-1. Learning rates for electric power generation and related technologies showing the percentage reduction in unit capital cost (or O\&M cost) for each doubling of cumulative installed capacity or production, based on peer-reviewed literature sources.

\begin{tabular}{|c|c|c|c|c|c|c|}
\hline \multirow{2}{*}{ Energy Source and Technology } & \multirow{2}{*}{$\mathbf{N}^{a}$} & \multicolumn{4}{|c|}{ Learning Rate ${ }^{b}$} & \multirow{2}{*}{ Source } \\
\hline & & Range & Mean & Error ${ }^{c}$ & Units & \\
\hline \multicolumn{7}{|l|}{ Coal } \\
\hline Pulverized coal plant (PC) & 4 & $5.6 \%$ to $12 \%$ & $8.3 \%$ & & GW & [27] \\
\hline FGD system & 1 & & $11 \%$ & & GW & {$[26]$} \\
\hline FGD system (O\&M cost) & 1 & & $22 \%$ & & GWh & [26] \\
\hline SCR system & 1 & & $12 \%$ & & GW & {$[26]$} \\
\hline SCR system (O\&M cost) & 1 & & $13 \%$ & & GWh & {$[26]$} \\
\hline Coal $^{b}$ & 1 & $-5 \%$ to $5 \%$ & $0 \%$ & & GW & [30] \\
\hline$P C+C C S^{b}$ & 2 & $1.1 \%$ to $9.9 \%$ & & & GW & [27] \\
\hline$I G C C^{b}$ & 2 & $2.5 \%$ to $16 \%$ & & & GW & [27] \\
\hline$I G C C+C C S^{b}$ & 2 & $2.5 \%$ to $20 \%$ & & & GW & [27] \\
\hline \multicolumn{7}{|l|}{ Natural Gas } \\
\hline NGCC & 5 & $-11 \%$ to $34 \%$ & $14 \%$ & & GW & {$[27]$} \\
\hline Gas Turbine & 11 & $10 \%$ to $22 \%$ & $15 \%$ & & GW & {$[27]$} \\
\hline$N G C C+C C S^{b}$ & 1 & $2 \%$ to $7 \%$ & & & GW & [27] \\
\hline$N G C C^{b}$ & 1 & $2 \%$ to $15 \%$ & $6 \%$ & & GW & [30] \\
\hline \multicolumn{7}{|l|}{ Nuclear } \\
\hline Nuclear plants & 4 & negative to $6 \%$ & - & & GW & [27] \\
\hline Nuclear plants & 3 & $-49 \%$ to $22 \%$ & $-44 \%$ & & GW & {$[30]$} \\
\hline Nuclear (business as usual) ${ }^{b}$ & 1 & $-25 \%$ to $0 \%$ & $-15 \%$ & & GW & {$[30]$} \\
\hline Nuclear (optimal conditions) & 1 & $0 \%$ to $10 \%$ & $5 \%$ & & GW & {$[30]$} \\
\hline \multicolumn{7}{|l|}{ Biomass } \\
\hline Power generation $^{d}$ & 2 & $0 \%$ to $24 \%$ & $11 \%$ & & GW & [27] \\
\hline Power generation & 1 & & $6 \%$ & & GW & {$[30]$} \\
\hline \multicolumn{7}{|l|}{ Hydroelectric } \\
\hline Hydroelectric plant & & & & & GW & [27] \\
\hline \multicolumn{7}{|l|}{ Fuel Cells } \\
\hline FCEV fuel cell stacks & & & $18.0 \%$ & $1.7 \%$ & GWh & [31] \\
\hline PEFC micro-CHP & & & $19.3 \%$ & $1.6 \%$ & No. units & [31] \\
\hline Fuel cells (residential) & & $16 \% \pm 2 \%{ }^{\mathrm{e}}$ & & & $\mathrm{GWh}_{\text {cap }}$ & {$[32]$} \\
\hline \multicolumn{7}{|l|}{ Hydrogen } \\
\hline $\mathrm{H}_{2}$ (alkaline electrolysis) & & & $17.7 \%$ & $5.3 \%$ & GW & [31] \\
\hline $\mathrm{H}_{2}$ (SMR) & & & $27 \%$ & & $10^{9}$ cu.ft. & {$[26]$} \\
\hline $\mathrm{H}_{2}$ (SMR) (O\&M costs) & & & $27 \%$ & & $10^{9} \mathrm{cu} . \mathrm{ft}$. & {$[26]$} \\
\hline
\end{tabular}




\begin{tabular}{|c|c|c|c|c|c|c|}
\hline \multicolumn{7}{|c|}{ Table 2-1. (cont'd) } \\
\hline \multicolumn{7}{|l|}{ Wind } \\
\hline Onshore & 12 & $-11 \%$ to $32 \%$ & $12 \%$ & & GW & [27] \\
\hline Onshore (1982-2016) & & & $5.9 \%$ & $1.3 \%$ & GW & [31] \\
\hline Onshore (2009-2016) & & & $24.5 \%$ & $2.1 \%$ & GW & [31] \\
\hline Offshore & 2 & $5 \%$ to $19 \%$ & $12 \%$ & & GW & [27] \\
\hline Offshore & & & $10.3 \%$ & $3.3 \%$ & GW & [31] \\
\hline Onshore $^{b}$ & & $-3 \%$ to $12 \%$ & $5 \%$ & & GW & [31] \\
\hline Offshore $^{b}$ & & $-5 \%$ to $10 \%$ & $3 \%$ & & GW & [31] \\
\hline \multicolumn{7}{|l|}{ Solar } \\
\hline PV system & & & & & GW & [27] \\
\hline PV systems & & & $18.6 \%$ & $1.0 \%$ & GW & [31] \\
\hline PV modules & & & $21.4 \%$ & $0.8 \%$ & GW & [31] \\
\hline PV balance of system (BOS) & & & $12.9 \%$ & $1.7 \%$ & GW & [31] \\
\hline CSP plants ${ }^{b}$ & 5 & $3 \%$ to $12 \%$ & $10.4 \%$ & & GW & [30] \\
\hline$P V(\text { short-term) })^{b}$ & 1 & $15 \%$ to $23 \%$ & $20 \%$ & & GW & {$[30]$} \\
\hline$P V\left(\right.$ long-term) ${ }^{b}$ & 1 & $8 \%$ to $17 \%$ & $12 \%$ & & GW & [30] \\
\hline CSP plants ${ }^{b}$ & 1 & $3 \%$ to $12 \%$ & $8 \%$ & & GW & [30] \\
\hline \multicolumn{7}{|l|}{ Energy Storage } \\
\hline Utility Li-ion storage & & & $15.2 \%$ & $3.7 \%$ & $\mathrm{GWh}_{\text {cap }}$ & [31] \\
\hline Utility redox-flow storage & & & $14.3 \%$ & $6.1 \%$ & $\mathrm{GWh}_{\text {cap }}$ & [31] \\
\hline Residential Li-ion storage & & & $12.5 \%$ & $3.0 \%$ & $\mathrm{GWh}_{\text {cap }}$ & [31] \\
\hline BEV battery packs & & & $15.2 \%$ & $2.9 \%$ & $\mathrm{GWh}_{\text {cap }}$ & [31] \\
\hline HEV battery packs & & & $10.8 \%$ & $0.6 \%$ & $\mathrm{GWh}_{\text {cap }}$ & [31] \\
\hline Pumped hydro (utility) & & $-2 \% \pm 8 \%^{e}$ & & & $\mathrm{GWh}_{\text {cap }}$ & [32] \\
\hline \multicolumn{7}{|l|}{ Other Technologies } \\
\hline Heat pumps & & & $10.0 \%$ & & No. units & [31] \\
\hline LNG production & & & $14 \%$ & & $\mathrm{Mt} / \mathrm{yr}$ & [26] \\
\hline LNG prod. (O\&M costs) & & & $12 \%$ & & $\mathrm{Mt} / \mathrm{yr}$ & [26] \\
\hline Oxygen production & & & $10 \%$ & & $10^{9}$ cu.ft. & [26] \\
\hline Oxygen prod. (O\&M costs) & & & $5 \%$ & & $10^{9}$ cu.ft. & {$[26]$} \\
\hline \multicolumn{7}{|l|}{ Energy Efficiency } \\
\hline Specific energy use (GJ/t) & & $12 \%$ to $29 \%$ & & & $\begin{array}{l}\text { Mt } \\
\text { product }\end{array}$ & [33] \\
\hline
\end{tabular}

a Number of studies. Some studies report multiple values based on different datasets, regions, or assumptions ${ }^{b}$ LR values in italics reflect model estimates or other projections, not historical data; all values are reported as percentages rather than decimals; negative learning rates indicate increasing (rather than decreasing) costs; "Units" refers to $x$-axis quantity.

' Standard error equals square-root of the diagonal of the variance-covariance matrix.

${ }^{\mathrm{d}}$ Includes combined heat and power systems and biodigesters.

e $95 \%$ standard error confidence interval

Note too that learning rates, properly derived, relate cost reductions with increasing experience in doing the same "job"-such as installing a unit of power plant capacity, generating a unit of electricity, or capturing a fixed percentage of air pollutant emissions (e.g., 90 percent $\mathrm{SO}_{2}$ capture using an FGD system). If the nature of the job changes - such as capturing only 50 percent $\mathrm{SO}_{2}$ instead of 90 percentthe historical learning rates in Table 1-1 may no longer apply. Similarly, negative learning rates typically reflect changing technology design requirements. 
Table 1-2. Additional learning rate estimates based on expert judgment for selected technologies [23]

\begin{tabular}{|c|c|c|c|}
\hline Cost Category/Technology Type & Learning Rate & Cost Category/Technology Type & Learning Rate \\
\hline \multicolumn{2}{|l|}{ Category 1} & \multicolumn{2}{|l|}{ Category 6} \\
\hline Coal Delivery and Handling & $1 \%-4 \%$ & Advanced Combustion Turbines & $4 \%$ \\
\hline \multicolumn{2}{|l|}{ Category 2} & Syngas Combustion Turbines & $5 \%$ \\
\hline Coal Prep and Feed & $1 \%-4 \%$ & Hydrogen Combustion Turbines & $5 \%$ \\
\hline \multicolumn{2}{|l|}{ Category 3} & Natural Gas Combustion Turbines & $1 \%$ \\
\hline Feed Water/Misc. BOP & $1 \%-5 \%$ & \multicolumn{2}{|l|}{ Category 7} \\
\hline \multicolumn{2}{|l|}{ Category 4} & Heat Recovery Systems & $1 \%$ \\
\hline Boiler Equipment \& Aux. & $1 \%-9 \%$ & \multicolumn{2}{|l|}{ Category 8} \\
\hline Gasifier Systems & $4 \%-21 \%$ & Steam Turbines & $1 \%$ \\
\hline Syngas Cooling & $4 \%-21 \%$ & Advanced Steam Turbines & $4 \%$ \\
\hline Air Separation Units & $3 \%-15 \%$ & \multicolumn{2}{|l|}{ Category 9} \\
\hline \multicolumn{2}{|l|}{ Category 5} & Cooling Towers/Systems & $1 \%$ \\
\hline Syngas Clean-up & $3 \%-15 \%$ & \multicolumn{2}{|l|}{ Category 10} \\
\hline Acid Gas Removal & $3 \%-17 \%$ & Ash/Slag/Spent Sorbent Handling & $2 \%$ \\
\hline Particulate Removal & $3 \%-17 \%$ & \multicolumn{2}{|l|}{ Category 11} \\
\hline Mercury Removal & $3 \%-17 \%$ & Power Distribution System & $1 \%$ \\
\hline Warm Gas Clean-up & $3 \%-17 \%$ & \multicolumn{2}{|l|}{ Category 12} \\
\hline Sulphur Recovery & $3 \%-17 \%$ & Instruments \& Controls & $1 \%$ \\
\hline Flue Gas Clean-up & $2 \%-17 \%$ & \multicolumn{2}{|l|}{ Category 13} \\
\hline Particulate Removal & $2 \%-17 \%$ & Site Preparation & $1 \%$ \\
\hline $\mathrm{SO}_{2}$ Removal & $2 \%-17 \%$ & \multicolumn{2}{|l|}{ Category 14} \\
\hline $\mathrm{NO}_{\mathrm{x}}$ Removal & $2 \%-17 \%$ & \multirow[t]{10}{*}{ Buildings \& Structures } & $1 \%$ \\
\hline Mercury Removal & $3 \%-17 \%$ & & \\
\hline Syngas Conversion & $3 \%-5 \%$ & & \\
\hline Fischer-Tropsch Synthesis & $5 \%$ & & \\
\hline Methanol \& Ethanol Production & $3 \%$ & & \\
\hline $\mathrm{CO}_{2}$ Capture, Recovery & $3 \%-18 \%$ & & \\
\hline $\mathrm{CO}_{2}$ Compression & $5 \%-10 \%$ & & \\
\hline Fuel Cells & $2 \%-6 \%$ & & \\
\hline Direct Liquefaction Process & $6 \%$ & & \\
\hline $\mathrm{CH}_{4}$ From Hydrates & $6 \%$ & & \\
\hline
\end{tabular}

Table 1-2 shows additional estimates of learning rates for technologies and plant components that lack modelled or historically-based learning rate studies. Accordingly, these rates are based primarily on the expert judgment of power-sector cost estimators, with upper bounds informed by selected literature on energy technology learning rates [23]. Such estimates may be useful in the absence of retrospective or prospective learning rates for a technology of interest. The learning rates derived from historical experience curves are commonly employed to address Type 2 (What Will) questions about the expected future cost of an advanced technology once it is widely deployed commercially. Section 1.5 discusses the selection and application of learning rates to carry out such projections, including estimates of expected $N^{\text {th }}$-of-a-kind costs. 


\subsubsection{Hybrid method}

The hybrid costing method introduced in recent work [3] refers to a combination of the engineeringeconomic and experience curve methods of cost estimation discussed above. It was developed to address Type 2 questions for advanced technologies. The section describes the method and its use.

\subsubsection{Combining bottom-up and experience curve analyses}

The use of an experience curve to project the future cost of a technology requires as its starting point the current cost of a commercial-scale system. In the hybrid method, a bottom-up engineering-economic analysis is thus used to first estimate the current capital and operating costs of a full-scale commercial project. For an advanced technology that has not yet been built at scale, this would represent the FOAK cost for a plant employing that system.

From this starting point, an experience curve is then used to project future cost reductions as the technology is further deployed. The need for experience in building and operating full-scale plants is inherent in the experience curve model since cost reductions are a function of the cumulative installed capacity or production. Cumulative capacity also reflects the time it takes to learn from experience with multiple generations of a technology as it evolves. After a sufficient amount of deployment with decreasing levels of change, the technology can be considered to be mature, corresponding to the concept of an NOAK plant.

\subsubsection{Illustrative example for Type 2 questions}

Figure 1-6 illustrates the use of the hybrid method and the type of results derived from this approach. This is an ideal case where the first commercial installation successfully achieves all performance requirements. As the cost of the advanced technology then declines from its current (FOAK) value (at installed plant capacity, C1), the analysis reveals the cumulative installed capacity, C2, needed to match the current lower cost of a competing baseline technology that is currently available commercially. Continuing down the curve shows the additional level of experience, C3, needed to achieve a still lower cost target or goal. Similar curves for other advanced technologies can allow one to identify the technologies that are likely to meet cost goals most quickly.

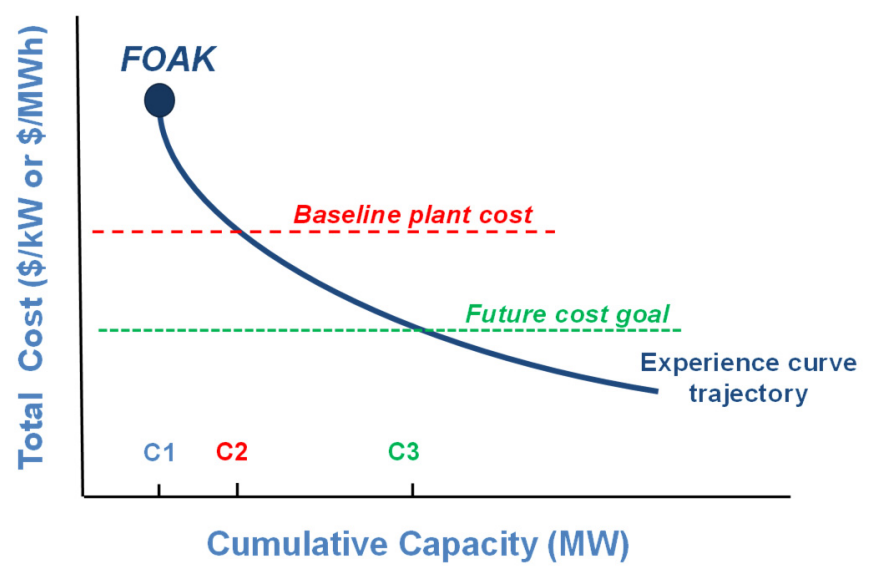

Figure 1-6. Illustrative cost trajectory of an advanced technology from FOAK plant to mature plant showing the deployment of the technology needed to meet a given cost goal.

Note that this formulation does not have an explicit value of NOAK cost since there is no unique value of $N$ (or cumulative capacity) at which a technology is considered to be mature. As a rule of thumb, however, the cost trajectories in Figure 1-4 show that cost tends to level out and decline slowly once cumulative experience reaches about 15 to 20 replications ( 4 to 5 doublings) of the initial capacity. At that point, a technology can reasonably be labelled as mature, as it is well-established and likely being offered by multiple vendors competing for future sales. For a mid-value learning rate of 0.15 the cost after four 
doublings has fallen to roughly half its initial value. Note too that the area under the curve between any two points represents the total plant investment needed to go from one point to the other.

\subsubsection{Strengths and limitations of alternative methods}

The three costing methods described above have different strengths and weaknesses depending upon the uses to which they are put and the questions they are employed to address. In the context of cost estimates for advanced power plants and other energy conversion technologies, as well as low-carbon industrial processes, the strengths and weaknesses of each method are highlighted below.

\subsubsection{Engineering-economic method}

In general, the strength of the engineering-economic method is that it can provide a rigorous and accurate cost estimate for a well-defined process or plant design if employed with appropriate data and parameter assumptions. Thus, as noted earlier, it is appropriate to address what-if questions about the overall cost of a plant whose design, performance, and component costs are fully specified. For advanced technologies, such specifications typically represent a hypothetical mature (NOAK) plant design that meets specified performance and component cost targets.

Conceptually, this method is best suited to estimate the current cost of a commercial project that would be built now or in the very near future-i.e., what-will questions about the current cost of a technology or its next commercial offering. It is primarily intended (and most widely used) for costing a facility employing technologies that are already in commercial use. For an advanced technology that has not yet been built and operated at scale, the output of this method would be the FOAK cost. That cost estimate would have to rely on what is currently known about the technology's performance and other attributes such as the cost of novel components. A higher degree of technical and professional judgment is also required since reliable information is typically lacking for early-stage technologies (such as the cost of fabricating a unique piece of equipment that has never been built at scale). The FOAK cost estimate for a new technology is thus inherently more uncertain than one for a more mature technology, resulting in more conservative assumptions and higher contingency costs (as elaborated later in this paper). The methodological approach of the bottom-up method is nonetheless appropriate for this application.

The principal weakness of the engineering-economic method in the context of this chapter is that is not suited or appropriate for addressing what-will questions about the future mature-plant cost of an advanced technology that is still under development. As noted earlier, that is because one cannot know today what the future design, performance, and component costs will be of a new technology that is many years (typically decades) away from the concept of an NOAK facility. Rather, future NOAK plant designs and technology component costs evolve with experience and cannot be established reliably without first deploying and operating multiple commercial plants over a period of time. Thus, a bottom-up analysis of a proposed future design is essentially a "what-if" exercise that simply assumes that the future NOAK design, performance, and cost assumptions are viable and will be achieved. Effectively, it is an estimate based on the judgments and opinions of the analysts or other experts who conduct the analysis.

Another significant shortcoming of a bottom-up analysis for a future NOAK plant is that it does not provide a timeframe in which the future performance and cost are expected to be achieved. At best, a time dimension might be estimated based on the judgment of the analyst or others involved in the cost estimate. While experience curves also do not provide an explicit measure of time, the cumulative installed capacity is a surrogate that can be linked directly to elapsed time via a deployment scenario or past experience with the rate of technology deployment.

\subsubsection{Experience curve method}

The principal strength of the experience curve method is that it is grounded in actual historical experience on the rates and magnitudes of cost reductions for technologies that are successfully adopted and deployed in the marketplace. Further, by linking cost reductions to cumulative deployment of a particular technology, it implicitly brings time into the picture as a key variable characterising cost trends. The 
relationship between cumulative capacity and time also can be made explicit via energy-economic modelling (e.g., van der Broek, et al., 2009) in which advanced technologies are deployed in the context of a particular scenario, such as a "business as usual" reference case or a proposed policy scenario. Thus, experience curves provide a dynamic model of cost reductions that evolve over time as technologies are deployed and continually improved. A schematic of that process of technological change is shown in Figure 1-7.

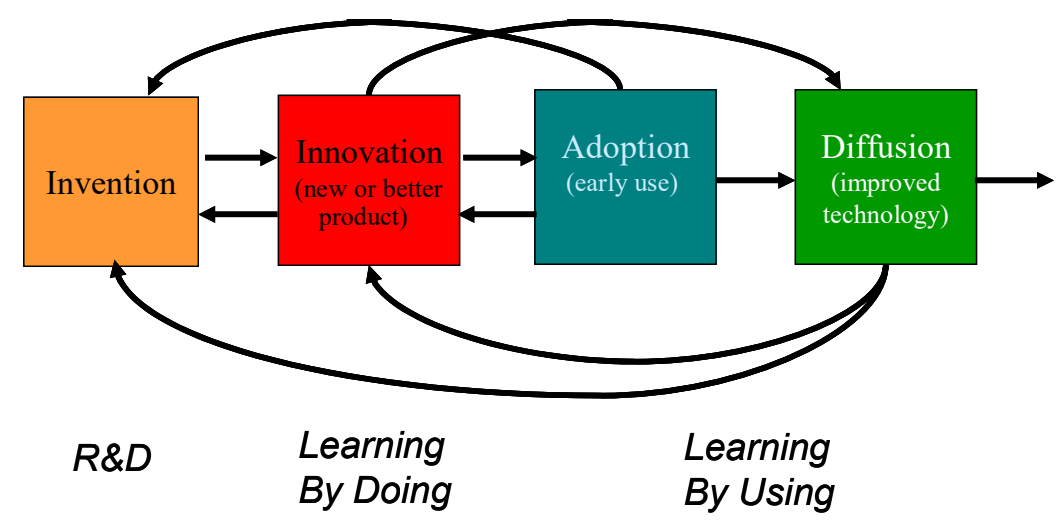

Figure 1-7. The dynamic process of technological change and innovation [34]

An acknowledged shortcoming of cost projections based on historical experience curves is that the single parameter on which most models are based (e.g., cumulative installed plant capacity) is effectively a surrogate that incorporates all other factors that influence cost reductions in addition to true "learning by doing" (which represents efficiencies in manufacturing and construction). Other factors include such things as the level of R\&D investments, scale economies, market conditions, and level of competition among firms [35]. Also, because of data limitations and availability, most experience curves are based on the price paid for a technology (i.e., the cost to the customer) rather than the cost of production or manufacture (which is the true measure that underlies learning by doing).

In addition, the experience upon which the learning rates are based may not necessarily apply to a different technology in the future. As seen in Section 1.2.2.2 above, there is also a wide variation in learning rates across the set of technologies that have been studied. Thus, the selection of a learning rate for a particular advanced technology cost projection is not straightforward. Guidance on such selections, however, is offered in Section 1.5 of this report, including how to deal with cases where an advanced technology involves a mix of novel and mature components. Uncertainties in experience curve rates and models, including their shape, are discussed in Section 1.6.

Another limitation is that cost projections based on learning rates offer no specific insights as to precisely why costs decline or how technology designs evolve and change over time. Thus, one can only estimate what a mature (NOAK) plant is expected to cost, not what it is likely to look like. However, past studies of energy and environmental technologies indicate that economies of scale, competition among firms, lower-cost manufacturing, knowledge sharing, and sustained R\&D are among the key factors that contribute to long-term cost reductions $[14,36]$.

Finally, any use of experience curves to estimate the future cost of an advanced technology requires an independent estimate of the current commercial cost of the technology and its level of deployment, from which cost reductions are assumed to begin. This need is addressed using the hybrid method discussed earlier. 


\subsubsection{Hybrid method}

The strength of the hybrid approach to cost estimates for advanced technologies is that it combines the strengths of the engineering-economic and experience curve methods to address what-will questions about future costs. Thus, a bottom-up engineering-economic analysis is first used to estimate the current cost of a commercially-deployed plant based on current information about the advanced technology design, performance, and cost. Section 1.4 of this chapter provides guidelines on the construction of that FOAK cost estimate. Next, an experience curve model is used to provide an empirically-grounded projection of the future cost trajectory, beginning with the current cost. This approach thus avoids the use of assumptions about the future technology design and the future cost of components or systems that have not yet been built and operated at scale. This approach also explicitly recognizes the critical role of experience with full-size plant construction and operation in achieving future cost reductions. Thus, the hybrid approach offers a critical parameter that is lacking in traditional bottom-up cost estimates, namely, a measure of the time (embodied by cumulative experience) needed to achieve significant cost reductions. The hybrid method can be applied to complex plant designs involving multiple components with different levels of maturity and different learning rates, as illustrated in Section 1.5.

As with its component methods, the hybrid method can be subject to significant uncertainties, both in the estimation of current (FOAK) plant costs as well as in the future cost trajectory for advanced technology components. The same would be true, however, of any other method of estimating the future cost of a technology. Section 1.6 discusses uncertainty characterisation in greater detail.

Finally, a limitation of the hybrid method is that it does not explicitly incorporate the potential for capital or other cost increases during early deployment, as seen in a number of historical case studies [26]. To the extent such increases occur (e.g., due to shortfalls in initial reliability or performance, or a lack of competitive markets), the proposed hybrid method may yield systematically optimistic cost projections by underestimating the true FOAK cost (as would other costing methods). Methods and guidance for dealing with this issue are discussed in Section 1.5.

\subsection{Guidelines for future cost estimates for Type 1 (What if) questions}

This section focuses on several key assumptions and inputs to a bottom-up cost analysis of a hypothetical new plant employing an advanced technology that is considered to be mature and widely deployed. Given that the methodological details of a bottom-up costing method are already well-documented (e.g., [6, 16, 19]), the guidelines in this chapter emphasize the need for assumptions that are consistent with the premise of the "NOAK cost goal" analysis. The following sections briefly highlight several areas where past studies were often deficient or inconsistent in the reporting of key assumptions.

\subsubsection{Plant and process design assumptions}

The starting point for any bottom-up cost analysis is the specification of plant and process flowsheets that establish the major pieces of equipment together with mass and energy flows for the given design. The study in Figure 1-2, for example, assumed an advanced coal gasification system with warm-gas clean-up coupled with a solid oxide fuel cell system, an ion transport membrane-based oxygen plant, and an advanced hydrogen-fired gas turbine. The overall plant was modelled in a commercial software system (Aspen Plus) to determine the mass and energy flows used to size all equipment for specified component performance characteristics.

For a "what if" analysis of a hypothetical mature plant using one or more advanced technologies, the principal guidance for plant design is to explicitly state all assumptions regarding the major plant equipment, battery limits, redundant equipment, and the size (and size limits) of major plant components. Equipment size limits might be expressed in terms of a maximum flow rate (mass or volumetric), or maximum equivalent generating capacity (MW), of a single vessel or item, given constraints on construction or commercial offerings. Depending on the size of the overall facility being 
analysed, multiple vessels or equipment trains may therefore be needed for some components. This has a direct impact on capital costs.

Some plant components also may be oversized to accommodate off-design conditions, and some equipment may require a degree of redundancy (sparing) to ensure reliability. Here too, such assumptions have a direct impact on plant cost. Studies must therefore state assumptions about equipment overdesign and redundancy or indicate clearly if all equipment is assumed to exactly meet maximum design flow rates with no redundancy.

Similarly, the principal guidance for plant and process performance is to explicitly state key design and operating assumptions for the assumed NOAK plant. This includes principal mass and energy flows; pollutant removal efficiencies and associated energy and reagent requirements of all major plant equipment; and specification of all plant products and product quality, where applicable. Other useful information includes descriptions and basis for system or process flowsheets, physical property models, and any other unique attributes of an advanced technology.

\subsubsection{Capital and O\&M cost assumptions}

Given the hypothetical nature of an NOAK cost estimate, authors have fairly wide latitude in the assumptions for underlying capital and operating cost parameters. Again, the key need is for consistency with the stated premise of the analysis, and transparency in the source or basis for component cost assumptions.

\subsubsection{Bare erected cost}

As indicated in Figure 1-1, the major building block for capital cost estimates is the bare erected cost (BEC) of the stipulated plant design. As noted earlier, for an NOAK plant, that cost is typically based either on specified (pre-established) cost targets for advanced technology components, or on cost estimates from other sources. Section 1.4.2 later provides additional guidance on the quantification of plant equipment costs. For the hypothetical plants in Figure 1-2, the BEC was based almost entirely on R\&D cost targets specified by DOE/NETL, as well as on "vendor-furnished projections for next generation technology" $[10,23]$.

However, most studies fail to sufficiently explain how the assumed BEC cost targets or projected values were determined, leaving it up to the reader to judge whether the resulting BEC is reasonable, achievable, or consistent with the methods employed in other studies of other technologies. Thus, the reported value of BEC for an advanced technology - which is the building block for total cost estimates is arguably the most poorly documented and least transparent component of an estimated hypothetical NOAK cost.

Thus, NOAK cost studies should always discuss the methods and data sources used to obtain BEC estimates. To avoid ambiguity about the cost basis, good practice also demands that the cost year be clearly (and repeatedly) reported, as well as the location to which the BEC estimate applies.

In this regard, the IEAGHG recently published a set of location-specific cost factors that give the relative costs of materials and labour in twelve geographic regions worldwide (see Table B-1 in Appendix B) [37]. Cost variations within a region also should be considered using other sources of country-specific data (for example, for U.S. power plants the IECM computer modelling tool adjusts the BEC for six geographic regions based on U.S. data for materials and labour costs) [38]. In addition, information on the current cost of advanced technology components should be provided to give context to the BEC estimates, and help readers assess the degree of progress required to achieve the assumed NOAK cost values.

Finally, to add a degree of conservatism and credibility to hypothetical NOAK cost estimates, we recommend that estimates of the future Bare Erected Cost of a mature technology take into consideration the actual current state of that technology. This is because the future BEC of a technology currently at a low TRL value is inherently more uncertain and speculative than that of a 
technology at a higher TRL. NOAK studies should therefore report whether (or how) such considerations are reflected in the BEC estimate. One approach would be to incorporate the cost adders recommended by NETL and EPRI as "process contingencies" (which vary by process maturity) directly into the BEC estimate for the hypothetical NOAK design. This would help temper the "technological optimism" that often characterizes BEC cost estimates for technologies that are still at an early stage of development. As discussed below, a subsequent process contingency factor would be applied based on the "what if" assumption of a mature technology. ${ }^{3}$

\subsubsection{Process contingency cost}

In bottom-up cost estimates, the process contingency cost is the capital cost added to the BEC to compensate for uncertainties associated with the development status of a technology based on its current level of maturity or development. The quantitative guidelines adopted by EPRI, NETL $[18,19]$ and others were developed many years ago by AACE International $[16,17]$ (see, [6]). Effectively, this is an estimate of the additional (miscellaneous) capital costs that are expected to be incurred in a near-term commercial project based on the current state of information and experience for the technology under study. Thus, the initial (FOAK) deployment of an advanced technology would incur a much higher process contingency cost than an established commercial technology, as elaborated later in Section 1.4.2.2.

However, in the case of a "what-if" NOAK cost estimate in which an advanced technology is assumed to be fully mature, the process contingency cost should be consistent with the premise of the analysisin this case, that of a mature commercial system. The NETL and EPRI guidelines cited earlier provide a numerical range of $0-10 \%$ for such estimates (see Table 1-5). While such values may seem unrealistically low given the actual development status of an advanced technology, it is nonetheless consistent with the premise of a mature technology whose future design and cost are assumed to be known today.

Note too that the project contingency factors in Table 1-5 do not depend on the degree of engineering detail underlying the analysis, as they do for project contingency factors (discussed below). Other recent studies of project cost estimates, however, suggest that higher process contingencies also should apply to less detailed preliminary analyses compared to more detailed studies for investment decisions (e.g., see Table B-2 in Appendix B) [40]. This methodological limitation of most current cost analyses tends to result in underestimates of true cost for the classes of study most commonly employed for advanced technology assessments.

To add a degree of conservatism to advanced technology cost estimates, some Type 1 NOAK studies assume the higher process contingency cost factor applicable to the current (less mature) status of the technology. However, that remains inconsistent with the definition of this factor and the premise of a mature technology for "What If" studies. Instead, as noted in Section 1.3.2.1, we recommend that this higher cost factor be incorporated (transparently) into the Bare Erected Cost of the advanced technology. Then, based on the assumption of a mature technology, a lower process contingency factor would be applied to the BEC, as prescribed by the engineering-economic methodology. This approach would also provide a measure of methodological consistency across studies of different advanced technologies.

Methodological consistency and transparency are especially important for technology cost comparisons, which are often the objective of NOAK cost studies. Often, however, studies fail to report their assumptions regarding contingency costs, resulting in a lack of transparency that leaves doubt as to whether contingency costs were even included in the analysis, and whether the reported costs are therefore biased downward. Clear and full reporting of contingency cost assumptions (as well as BEC

\footnotetext{
${ }^{3}$ The concept of Commercial Readiness Level (CRL) also has been developed to reflect the additional uncertainty and risk remaining in the deployment phase of commercialization due to factors such as the capabilities and capacity depth in supply chains, engineering services and other factors that may affect the cost of FOAK and subsequent projects. This has been applied mainly to renewable energy technologies where access to capital has also been a barrier to accelerated deployment [39].
} 
assumptions) thus remains critical. It is then up to the audience for such cost estimates (including reviewers and editors of technical journals) to judge their credibility.

\subsubsection{Project contingency cost}

The project contingency cost is an additional capital cost item that accounts for the cost increases that typically occur with increasingly detailed information on site-specific costs. The cost factor guidelines originally established by AACE International and subsequently adopted by EPRI and NETL, call for higher values in studies with less site-specific detail [16, 19, 41]. However, reviews of recent studies found that assumed project contingency cost factors are systematically low relative to established guidelinesespecially for "budget-type" cost estimates that are common for advanced technology studies [8, 9]. The result is to understate the estimated capital cost by as much as $10 \%-30 \%$. Thus, NOAK cost estimates should be careful to include the project contingency cost factors that are consistent with the level of site-specific detail of the study.

\subsubsection{Other capital cost factors}

Figure 1-1 lists the additional elements of a capital cost estimate, which include pre-production costs, inventory capital, financing costs, capital cost escalations and interest on debt during construction, and other "owner's costs" that are enumerated in guideline documents for engineering-economic cost estimates (e.g., [4, 6, 16, 19]). Readers should consult those documents for more detailed guidance on how to quantify these factors for commercial technologies. Again, for Type 1 questions the values chosen should be consistent with the premise of a mature NOAK technology.

\subsubsection{Operating and maintenance costs}

O\&M costs can be a significant component of overall cost, especially for advanced technologies that require large amounts of energy for their operation, or which use new materials not currently manufactured in large quantities. Examples include advanced $\mathrm{CO}_{2}$ capture processes employing large temperature or pressure swings, or which employ advanced materials such as ionic liquids, metal-organic frameworks, solid sorbents, or new types of membrane materials.

The prevailing assumption for O\&M costs in NOAK studies is that advanced technologies achieve specified performance and cost targets (e.g., for new materials). Seldom, however, do studies report the current cost of such materials or technologies, or discuss the means whereby the future cost goals are expected to be achieved. Thus, it is again left to the reader to judge whether assumptions about future cost are reasonable in the context of a particular study. As good practice, the rationale for assumptions about $O \& M$ costs should be discussed and justified in order to assist readers in judging the credibility of an NOAK cost estimate. For a complete list of O\&M cost factors and guidance on quantification readers should again consult the reference cited above in Section 1.3.2.4.

\subsubsection{Financing and plant utilisation assumptions}

For either power plants or other industrial processes, the total capital requirement of the project is amortized over the expected life of the plant and then combined with annual O\&M costs to calculate an overall cost per unit of product, either on a year-by-year basis or, more commonly, on a levelised annual basis. For power plants the common metric is the levelised cost of electricity (LCOE). For other industrial processes it is the levelised cost of product (LCOP). For plants that produce multiple products a principal product must be identified. Two key parameters for this calculation are highlighted below.

\subsubsection{Weighted average cost of capital}

The weighted average cost of capital reflects the overall cost of money borrowed or invested to finance a project using various sources of debt and equity financing. Together with the project lifetime, this rate determines the capital charge rate (also called fixed charge factor, FCF) that is used to amortize the total capital cost. Note that these rates can be expressed either in nominal (current money) terms that include an assumed rate of inflation, or in real (constant money) terms that exclude inflation effects. While the 
latter is most common and recommended for technology comparison studies (e.g., [6]), some studies employ nominal rates. Thus, it is critical that studies clearly report the cost basis used.

In general, the weighted cost of capital reflects the degree of risk associated "with a project—riskier projects involving new or early-stage technologies typically demand higher financing rates than mature, established technologies that have been widely replicated. By definition, however, NOAK projects are assumed to be mature, low-risk technologies that enjoy a low-risk cost of capital. In some past studies, however, the assumed cost of capital for an advanced NOAK technology was based on a high-risk project, which is inconsistent with the NOAK premise of a mature system. The result is to overstate the cost of the assumed NOAK plant. Cost of capital assumptions should be consistent with the assumed premise of the study.

\subsubsection{Plant capacity factor}

The annual average capacity factor (CF) of a power plant reflects the fraction of electricity actually generated in a year relative to the maximum possible generation if operated for a year at the full rated capacity. The value assumed for CF is arguably the most important parameter affecting the unit cost of electricity $(\$ / \mathrm{MWh})$ since it determines the amount of electricity over which the capital investment is spread. Thus, the higher the CF, the lower the cost per unit of electricity generated. Depending on how a power plant is operated (e.g., load-following vs. on-and-off at full capacity), lower capacity factors could also increase the plant heat rate (efficiency), and thus its LCOE.

For purposes of this report, guidance for NOAK studies again emphasizes the importance of CF assumptions that are consistent with the study premise, including assumptions about how the NOAK plant is operated. This guidance also emphasizes that for LCOE calculations, the assumed CF should be the levelised value over the life of the plant. This weighs initial years of operation more heavily than later years because of discounting (see, e.g., [8]).

Historical data on the capacity factor of mature coal-fired and gas-fired power plants over their operating life offer one credible benchmark for numerical assumptions about the future $\mathrm{CF}$ of a new NOAK plant. Although time series data on the $\mathrm{CF}$ individual generating units over their full operating life are not readily available, one case study of a large coal-fired unit reported a 25 -year average capacity factor of $77 \%$ from 1981-2005, with a levelised CF of $71 \%$ due to the characteristically lower CF during the first years of operation [8].

The U.S. Energy Information Administration (EIA) also publishes aggregated capacity factor data for U.S. power plants on a monthly and annual basis. Such data show, for example, that for coal-fired units the average plant capacity factor from 2010 to 2019 fell from $67 \%$ to $48 \%$, while for natural gas combined cycle (NGCC) units the average capacity factor rose from $44 \%$ to $57 \%$ [42]. Thus, over the past two decades, the utilization of both coal and natural gas plants has been well below their annual average availability of approximately $85 \%$ [43]. This applies also to large newer coal units, whose mean CF in any year was approximately five percentage points higher than the overall coal plant average based on data from 1998 to 2009 [8].

Other parts of the world show similar CF trends that are well below the typically assumed availability values of $85-90 \%[44,45]$. Looking ahead, power plants with and without CCS are expected to continue to have gradually reducing capacity factors as the grid share of intermittent renewable energy sources increases in the future (e.g., [46]).

Assumptions about the operating hours of a hypothetical NOAK plant therefore require careful consideration and justification, especially if the assumptions depart significantly from historical trends. Many recent cost studies, for instance, assume that power plant capacity factor is equal to the plant availability, meaning that the unit is dispatched at its nameplate capacity any time it is available (e.g., [12]). One rationale for this assumption is that the study objective is "to compare technologies based on their performance and cost merits without imposing market forces that would impact CF" [43]. Thus, 
in view of the historical data noted above, an assumption that the levelised capacity factor over the life of a plant is equal to its availability is a bounding case that yields the theoretical minimum value of LCOE for the technology analysed. Based on historical data, the actual expected CF would be lower and the estimated LCOE higher. This distinction between expected and bounding values of CF and LCOE should be clearly acknowledged in NOAK cost study results that employ that assumption. Failure to highlight this distinction can easily lead to misunderstandings that would bias comparative cost results and technology rankings based on LCOE.

\subsection{Guidelines for FOAK Cost for Type 2 (What will) questions}

As outlined earlier in Section 1.2.4.3, the starting point for the hybrid approach to addressing "What Will" questions about the future cost of advanced technologies is an estimate of the current cost of a commercial plant employing the technology. For an advanced technology that has not yet been built at a commercial scale, this would be the FOAK plant cost. In other cases, it would be the "next-of-a-kind" commercial project. In either case, the methodological approach is a bottom-up engineering-economic cost estimate similar to the one described in Section 1.3 for a mature plant, except that now all cost parameter values are based on a first-of-a-kind (or next-of-a-kind) plant design.

This section of the chapter highlights a number of areas where FOAK design and cost factors often differ significantly from NOAK estimates. The guidelines presented here draw heavily on a recent report on FOAK cost estimation prepared for NETL by Black \& Veatch, an engineering, procurement, consulting and construction company with broad experience in the electric power sector [47]. The FOAK guidelines also draw on the existing guidelines for conventional power plant cost estimation $[16,19]$, as well as on recent experience with FOAK projects employing power plant $\mathrm{CO}_{2}$ capture or other large-scale energy technologies [40, 48, 49]. Wherever possible, quantitative guidelines are provided in addition to qualitative discussions related to FOAK cost estimates.

In general, FOAK cost estimates are most appropriate for technologies that have advanced to at least a large pilot plant or full-scale testing of the new process or system (e.g., TRL 6 or 7 ) to provide the basis for further scale-up and integration for a full-scale commercial facility [47]. Though FOAK cost estimates also may be carried out for technologies at earlier stages of development, such technologies are inherently much riskier. Because of their higher risk, FOAK cost estimates must therefore also reflect the greater difficulty such projects would face in obtaining the financial and technical resources needed to actually undertake a commercial-scale project.

As a starting point, a number of initial screening questions can help frame cost estimates for advanced (pre-commercial) technologies [47]. For example: How long has the technology been in development? What is its current level of maturity? How have the risks associated with design, construction and operation of the technology been fully characterized and incorporated into the design and cost elements? Appendix A provides a more comprehensive list of screening questions and their relevance to FOAK cost estimates.

\subsubsection{Plant and process design factors}

As with cost estimates for mature plants, estimates for FOAK plants begin with a specification of the plant and process designs and the battery limits of the project. For FOAK or other early-stage projects, the design is likely to include a greater degree of spare or redundant equipment, oversized vessels and other items that would not be found in mature plants, but which are incorporated in early designs to help ensure reliable operation and desired performance. While such measures do not guarantee that the FOAK plant will indeed operate reliably as designed, the risk of failures or shortfalls are minimized by a more conservative design in the absence of prior (or limited) experience. 


\subsubsection{Equipment Sizing, Overdesign, and Redundancy}

Development of the FOAK design basis typically involves three phases [47]. The first is equipment sizing based on design flows, pressures, and other sizing-related measures. Next, an overdesign (or margin) is applied to ensure flexibility, safety, and resiliency in system operation. Typically, these design margins also account for operation at various ambient temperatures, and for and differences in feedstock properties (especially for coal-based plants). Lastly, redundancy is provided as additional protection for critical equipment in order to avoid forced outages and unnecessary downtime.

The most common applications of design margins are for essential equipment which must operate constantly, such as pumps, fans, and valves. Certain pieces of critical equipment, including large rotating machineries such as power plant steam turbines and flue gas fans, have an additional need for redundancy in some of their components. For example, to avoid potentially catastrophic equipment failure if oil flow to the bearings of a large steam turbine is interrupted, the lubricating and hydraulic oil skid for the turbine often utilizes $2 \times 100 \%$ or $3 \times 100 \%$ redundancy for motors, filters, and bypass valves. Similarly, large centrifugal or axial fans are typically designed for $2 \times 100 \%$ redundancy for critical motors, valves, and bypass lines [47]. ${ }^{4}$ In these cases, redundancy is applied to mature technologies as well as to FOAK installations.

However, for advanced technologies being built at scale for the first time, or with little prior operating experience, the need for spare (redundant) equipment to reduce risks is typically far greater than for mature technologies. Thus, early-stage technologies commonly employ redundancy not only for critical items like pumps, values, and fans, but also for major vessels that are critical to the operation of the new technology. For example, early deployments of wet flue gas desulfurization (FGD) systems at large coalfired power plants in the 1970s frequently employed a spare absorber train in addition to several operating trains because of reliability concerns. Similarly, many proposed designs for early-stage integrated coal gasification combined cycle (IGCC) power plants employed a spare gasifier along with several operating units. Only as process designs and scale improve with cumulative deployments and operating experience does the need for equipment redundancy decline or disappear.

The need for redundancy of FOAK technology or sub-systems with little or no operating experience at the scale envisioned should be determined based on a review of the factors that might cause a shutdown or poor performance of the technology. Such considerations would include the degree of scale-up, the duration and results of previous testing under a range of operating conditions, and the depth of technical understanding of the factors that affect the performance and reliability of the technology.

Inevitably, decisions about redundancy in FOAK designs reflect a balancing of risks (e.g., the consequences of a malfunction or failure of the technology to operate as expected) versus the added cost of spare equipment that will not be utilized if all goes as planned. A careful vetting of the proposed design can help identify critical needs for additional process development to avoid unnecessary redundancy that inflates the project cost estimate [47].

For illustrative purposes, the following are examples of typical redundancies for commercial-scale plant equipment [47]:

- 1x100\%. No redundancy; typical for large plant components that are mature and proven in prior experience, as well as for small blowers, non-critical valves, and tanks.

- $2 \times 50 \%$. No redundancy, since a single unit cannot carry the full rated load; however, partial service is maintained if one unit fails. This is most commonly used for pumps or blowers that cycle on and off periodically and are not critical to plant operation.

- 3x50\%. Partial (50\%) redundancy; however, a risk that no single unit can fully support the process if two of the units should fail simultaneously.

\footnotetext{
${ }^{4}$ A designation of $N \times 100 \%$ means $N$ pieces of equipment each capable of handling $100 \%$ of the flow. Thus, $2 \times 100 \%$ means one operating unit plus one spare unit that can be put into service if the operating unit fails.
} 
- $2 \times 100 \%$. High degree of redundancy and a common configuration for critical pumps, fans, and/or safety valves (with an option for both components to be run at half capacity for faster response to transient conditions).

- $\mathbf{3 x 1 0 0 \%}$. Potentially excessive redundancy, but could be warranted for a pump or compressor train that is critical for the safety of personnel and plant equipment.

For advanced technologies that have not yet reached commercial scale, FOAK designs for a full-scale plant may require the use of multiple vessels or trains due to limits on the size of available FOAK or earlystage equipment. For example, in the case of FGD systems noted above, early commercial equipment was often limited to flue gas flow rates equivalent to roughly $150 \mathrm{MW}$ of power plant capacity. A $450 \mathrm{MW}$ plant therefore required at least three operating absorbers for $\mathrm{SO}_{2}$ capture. Due to reliability concerns, a spare train was often added to early designs (i.e., 33\% redundancy). Similar levels of redundancy may be appropriate for advanced technologies with analogous characteristics.

In other cases, a novel technology at commercial scale may require dozens or hundreds of modules, reactors, or vessels operating in parallel. In such cases, a significantly lower level of redundancy would be expected based on the known or anticipated failure rate of individual modules. For example, a process that required 200 vessels operating in parallel at full load might be designed with only five spare vessels ( $2.5 \%$ redundancy) to provide enough redundancy to operate through unplanned equipment outages or malfunctions, while also promoting cost-effectiveness Black \& Veatch [47].

In summary, as illustrated in the examples above, there are no hard-and-fast rules for the precise level of redundancy in FOAK designs. Rather, each project requires an evaluation based on the criticality of process components and sub-systems, the extent of prior testing and experience, and the cost of critical technologies. In all cases, however, an explicit redundancy philosophy should be provided in all cost studies to ensure that this issue has been carefully considered and addressed appropriately.

\subsubsection{Other Factors Affecting FOAK Plant Design}

The following items are recommended best practices for contract management and process design for a pre-commercial or FOAK technology cost estimate. All of these factors apply as well to a Type 1 (aspirational NOAK) cost estimate, though the time and cost implications are different for FOAK and NOAK projects. The discussions of each item were provided by Black \& Veatch [47]:

- Target standard materials in process design. While hybrid or specialized materials can be attractive due to their unique or superior properties, material pricing, supplier availability and longterm maintenance considerations may indicate that other standard materials provide a more robust design option.

- Understand applicable codes and standards. Early alignment of the FOAK design with the National Electric Code (NEC), National Fire Protection Association (NFPA), International Building Code (IBC), and other applicable codes will avoid potentially costly design iterations and expedite third-party assessments and due diligence reviews.

- Incorporate realistic operability measures. These include controls, feedback measures, and safety protections so that when forced outages and equipment malfunctions occur the system has built-in methods to safely recover and operate until the situation is mitigated.

- Identify critical legal and contractual mechanisms needed to mitigate performance and schedule risks. A firm strategy for how these contractual items will be accounted for during project execution for FOAK technology is important for financing and insurance underwriting entities. Common items between equipment suppliers and end-users needed to secure a final contractual agreement include: performance and/or schedule liquidated damages; performance guarantees; limitations of liability; and, warranty periods. 
- Perform a hazard and operability analysis of the plant and/or process. This provides evidence that design risks have been mitigated and that safety has been designed into the plant. In particular, the concept of "safety by design" has gained rapid adoption in the industry [47].

- Inclusion of start-up, shut-down systems. This is an important requirement that is often overlooked in FOAK designs.

Another critical factor for a pre-commercial technology is the level of risk associated with the project design and construction schedule. Table 1-3 outlines critical FOAK technology project risk areas that should be considered when developing the design and execution plan for a facility incorporating precommercial technology [47]. In general, a higher level of risk results in higher project costs. The following sections of the chapter discuss more fully the cost items that are influenced by the design factors and risks identified in section 1.4.1. To help quantify the impact on plant cost of these (and other) systemwide risks, a new cost factor is introduced below in Section 1.4.2.3.

Table 1-3. Risk evaluation metrics for pre-commercial technology project planning [47]

\begin{tabular}{|l|l|l|l|}
\hline Risk Factor & Low Risk & Medium Risk & High Risk \\
\hline Project Schedule & $\begin{array}{l}\text { Sizeable project float } \\
\text { between engineering, } \\
\text { procurement, and } \\
\text { construction phases }\end{array}$ & $\begin{array}{l}\text { Aggressive schedule with } \\
\text { complex scope but adequate } \\
\text { float }\end{array}$ & $\begin{array}{l}\text { Minimal to no float between } \\
\text { project phases; all activities } \\
\text { are critical path }\end{array}$ \\
\hline $\begin{array}{l}\text { Technology Maturity } \\
\text { (Performance Risk) }\end{array}$ & $\begin{array}{l}\text { More than } 10 \text { successful } \\
\text { installations with more than } \\
1 \text { year of operation per site }\end{array}$ & $\begin{array}{l}3-10 \text { successful installations } \\
\text { with 0.5-1.0 years of } \\
\text { operation per site }\end{array}$ & $\begin{array}{l}\text { Less than } 3 \text { successful } \\
\text { installations with less than } \\
0.5 \text { years of operation per } \\
\text { site }\end{array}$ \\
\hline $\begin{array}{l}\text { Number of Sub-Suppliers } \\
\text { and/or Sub-Contractors }\end{array}$ & $\begin{array}{l}1-5 \text { equipment suppliers } \\
\text { within the project }\end{array}$ & $\begin{array}{l}\text { 5-10 equipment suppliers } \\
\text { with moderate-high } \\
\text { experience }\end{array}$ & $\begin{array}{l}\text { More than } 10 \text { separate } \\
\text { suppliers with low to } \\
\text { moderate experience }\end{array}$ \\
\hline
\end{tabular}

a "Float" refers to the time gap (margin) between the planned end date and the (earlier) critical path end date for a project activity.

\subsubsection{Capital cost factors}

Once the FOAK design is specified, the next step is to estimate its current capital cost based on the present state of technology development. Conceptually, this involves the same general procedure outlined earlier in Figure 1-1 for a bottom-up analysis, i.e., first estimate the bare erected cost of the plant, then apply other indirect cost factors and contingency costs to calculate the total capital requirement [16].

\subsubsection{Bare Erected Cost}

In general, the BEC for a first-of-a-kind project will be significantly greater than for a mature technology or a plant assumed to meet $R \& D$ goals. Key contributors are more conservative designs (including oversized and redundant equipment), longer construction schedules, and the use of new components or materials that are not yet widely fabricated or produced in large quantities or sizes.

The most robust method of developing a BEC estimate for a commercial project is to employ an independent third-party entity with experience in estimating construction and sub-contractor costs for large-scale construction projects. For a pre-commercial technology, this type of arrangement often can be protected by a non-disclosure agreement (NDA) which, in the U.S., would typically take 3-6 months at a cost of roughly $\$ 100,000$ to $\$ 200,000$, depending on the level of complexity and detail involved [47]. Most published cost estimates fail to include such costs, however.

If this option is not available or feasible (e.g., for a preliminary study), a BEC estimate for an advanced technology also can be developed based on guidance from cost estimation software (e.g., AspenTech [50]) 
as well as from a variety of widely-used textbooks and references on process equipment design and cost estimation (e.g., [51-53]). Where available, recent cost studies based on vendor quotes for other processes or equipment with similarities to the advanced technology under study also can be useful for bounding and scaling a BEC cost estimate. While the cost derived from any of these methods are subject to uncertainty (see Section 1.6), a careful effort to ensure that first-of-a-kind considerations are fully reflected in the BEC cost estimate is critical to this step-in cost estimation.

It is also helpful to compare the FOAK BEC estimates to those of current commercial technologies to help ensure that such estimates are reasonable and that all applicable plant design and equipment subcategories have been addressed. For power plant studies, for example, Figure 1-8 depicts the percentage breakdown of BEC components reported by Black \& Veatch [47] for commercial PC and NGCC plants without CCS recently analysed by NETL [43]. For advanced pre-commercial technologies, the BEC values in the relevant process area accounts would be expected to exceed the commercial system values for plants of similar overall design.

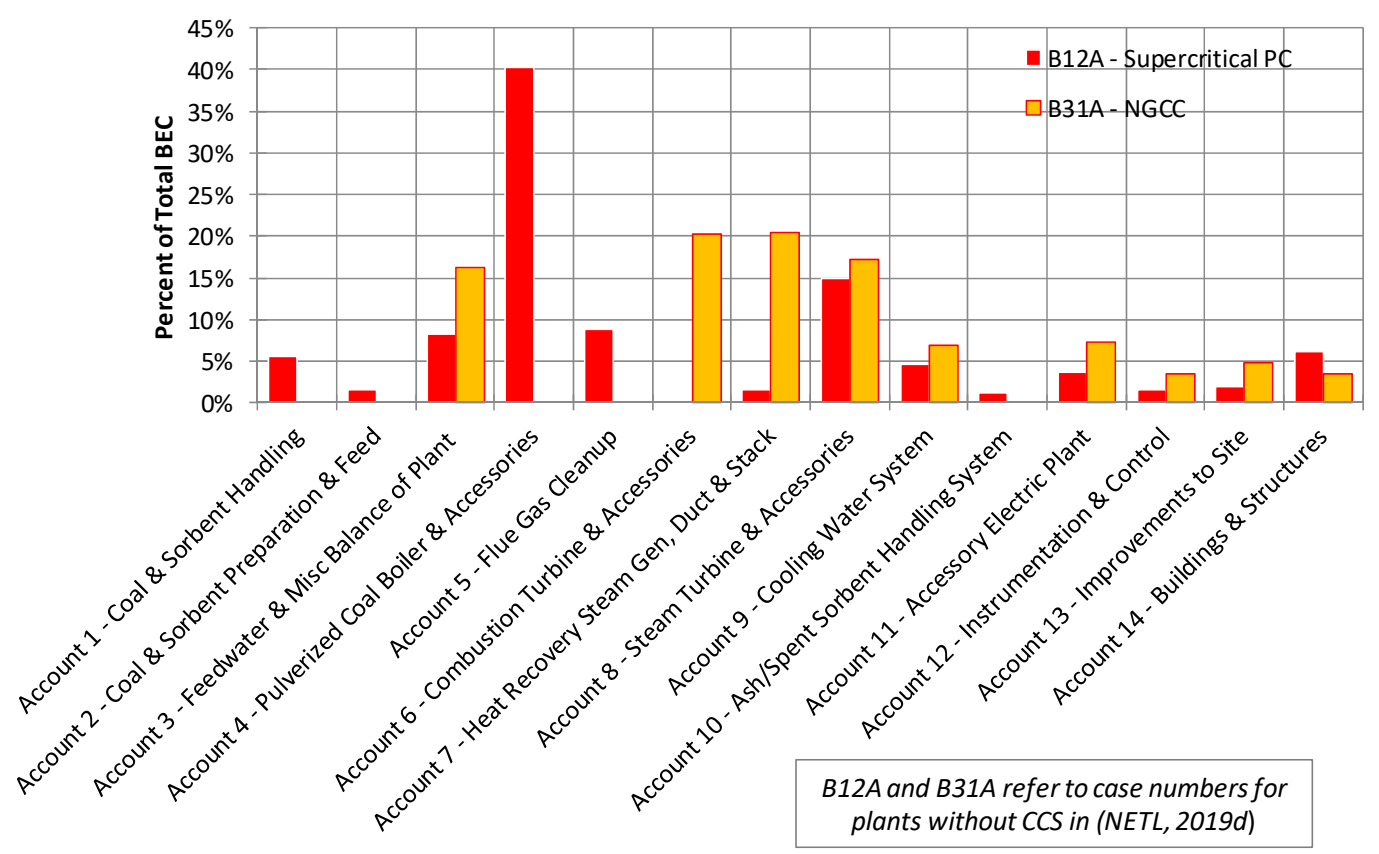

Figure 1-8. BEC breakdown by process area for commercial PC and NGCC plants [47]

\subsubsection{Process and project contingency costs}

As noted earlier in Section 1.3.2.2, capital cost estimates commonly include two types of contingency costs: process contingency and project contingency. The latter depends solely on the level of site-specific detail in the cost estimate (whatever the technology). Thus, guidance for project contingency cost estimates is the same for both FOAK and NOAK plants. See Section 1.3.2.3 for details or, e.g., AACE International [41].

In contrast, the process contingency cost differs significantly for FOAK and NOAK cost estimates since it depends on the experience base and maturity of the technology in question. As indicated in current cost guidelines $[16,19]$, the method of quantifying process contingency cost is to apply a percentage of the total installed cost of the process or technology in question. Table 1-4 shows the recommended values for processes at different levels of maturity. These guidelines, first developed by AACE International [17], also have been adopted by EPRI [18, 19]. Approximate TRL values corresponding to the AACE descriptions of technology status also are shown in Table 1-4. 
Table 1-4. Process contingency cost guidelines $[16,19]$

\begin{tabular}{|l|c|c|}
\hline Current Technology Status & $\begin{array}{c}\text { Indicative } \\
\text { Equivalent TRL } \\
\text { Value }\end{array}$ & $\begin{array}{c}\text { Process Contingency Cost } \\
\text { (\% of associated process } \\
\text { capital) }\end{array}$ \\
\hline New concept with limited data & $\sim 3$ & $40+$ \\
\hline Concept with bench-scale data & $\sim 4$ & $30-70$ \\
\hline Small pilot plant data & $5-6$ & $20-35$ \\
\hline Full-sized modules have been operated & $7-8$ & $5-20$ \\
\hline Process is used commercially & 9 & $0-10$ \\
\hline
\end{tabular}

Because advanced (pre-commercial) technologies vary widely in their complexity and degree of novelty, the contingency cost factors for each of the five development level categories in Table $1-4$ also have a wide range. Professional and technical judgments are thus required to select an appropriate value based on the current status of technology development and an assessment of the risks in scaling up to a commercial FOAK facility. An uncertainty analysis for capital costs would ideally encompass the minimum and maximum values judged to be appropriate for a particular study.

The key guidance conveyed by Table 1-4, however, is that in contrast to assumptions for NOAK cost studies, which commonly assume process contingency factors of zero to 10 percent (as for a mature commercial process), appropriate values of process contingency costs for pre-commercial FOAK studies are several times higher than for NOAK cost studies. The particular value selected may be more or less conservative, depending upon the judgment of the cost estimator and the purpose of the study.

Additional guidance provided by Black and Veatch [47] is that the process contingency factor for any individual component should seek to be less than 40 percent, as higher values would indicate that the process is not suitably defined or understood for a full-scale project. This perspective implies that the process status in Table 1-4 should be at least at the "small pilot plant data" level (e.g., TRL 5-6) to avoid excessive risks in FOAK cost estimates for a commercial-scale project. For processes currently at earlier stages of development, FOAK cost estimates based on more limited data nonetheless require much higher process contingencies, as indicated in Table 1-4.

Another independent perspective on contingency cost guidance is provided in a 2014 study that reviewed the actual and estimated costs for ten recent large-scale energy projects in Australia [40]. As a result, that study also recommended substantially higher process and project contingencies to bring initial cost estimates in line with actual final costs. The total contingencies recommended for projects at different stages of development and cost studies at different levels of granularity are generally higher than current NETL guidelines. That study further recommended an additional indirect cost item called "supplemental funds" to provide a financial buffer for project investors to deal with less likely "extraordinary situations" that may occur during the definition and/or implementation phase of a project (for example, a change in the project site, significant plant design changes, a need to re-route a $\mathrm{CO}_{2}$ pipeline, and/or changes in access to critical infrastructure such as high voltage transmission or plant water supply). These guidelines also were recently published in a peer-reviewed journal article and employed for an advanced technology cost estimate (Larsen, et al., 2020). Table B-2, in Appendix B, summarizes these guidelines as an additional reference for FOAK and NOAK cost estimates, and Figure B-1 (in Appendix B) shows some of the underlying data for these guidelines.

Because contingency cost values for FOAK projects often have broad ranges, the resulting cost estimates also have a higher degree of uncertainty than projects employing mature technologies. Section 1.6.1 discusses uncertainty and risk analysis methods to characterize those uncertainties, including probabilistic methods of evaluating contingency and overall project costs. 


\subsubsection{System Contingency Cost}

Advanced technologies often combine new or established process components in novel ways that have not yet been implemented at a commercial scale. One example is the IGFC power plant design in Figure 1-2, which integrates advanced gasifiers, oxygen plants, fuel cells, gas turbines, and other components into a unique system. Another example is a calcium looping $\mathrm{CO}_{2}$ capture system, which combines several commercial components in a new way [54].

In all such cases, increased system complexity and novel integration schemes introduce new risks that tend to reduce the overall reliability of FOAK installations. As a result, additional costs are likely to be required to achieve and maintain the design level of performance. Even where only a single new process is integrated into a conventional plant for the first time, interactions with other system components can cause unexpected problems and process outages that require additional expenses to rectify (e.g., [55]). These additional system-level costs can be significant but are not typically reflected in the process contingency cost factors described earlier, which depend only on the maturity of individual plant components (see Table 1-4).

To account for additional capital costs related to system integration and complexity, an additional contingency cost factor is introduced here, called system contingency cost. This factor is uniquely applicable to the initial installations of a new technology or system design, where integration-related problems first arise. It is similar in nature (though smaller in magnitude) to the "supplemental funds" factors discussed above and shown in Appendix B. It is also similar in nature (and magnitude) to the location-specific cost factor recently introduced by IEAGHG [37] to account for "additional potential execution risks" associated with the implementation of a large CCS project. Those factors are shown in the last column of Table B-1 in Appendix B.

Pending further data acquisition and analysis efforts to quantify this factor, the guidance in Table 1-5 is suggested by the authors of this chapter to ensure that this potential cost adder is not overlooked in FOAK cost estimates. The suggested value of this factor, informed by the studies of Greig, et al. [40] and IEAGHG [37], is greatest for the first commercial installation. The system contingency cost then decreases for subsequent projects, assuming that any systems-related problems found at a FOAK installation will result in design changes (and associated cost increases) to eliminate the problem in subsequent replications. To the extent that initial design changes are not wholly successful, some risk for subsequent early-mover projects may remain, pending demonstration that the reliability problem has indeed been resolved successfully. Thereafter (nominally after five replications of a project), the system contingency goes to zero. However, for any non-zero values of the initial (FOAK) system contingency cost the bare erected cost likely will have increased as a result of design changes in each of the early projects.

Table 1-5. Proposed system contingency cost guidelines

\begin{tabular}{|l|c|}
\hline Technology Status & $\begin{array}{c}\text { System Contingency Cost } \\
\text { (\% of total process capital for all } \\
\text { newly integrated components) }\end{array}$ \\
\hline First commercial-scale project (FOAK) & $0-20$ \\
\hline Second and third commercial projects & $0-10$ \\
\hline Fourth and fifth commercial projects & $0-5$ \\
\hline All subsequent commercial projects & 0 \\
\hline
\end{tabular}

A key distinction from the process contingency cost is that the system contingency cost factors depend only on the level of prior experience with the integrated system and not on the maturity of the individual technological components. Thus, mature technology components integrated in a unique fashion for the 
first time would have the same system contingency factor as advanced technology components that are combined for the first time. Since the system contingency cost factor has not been incorporated in prior engineering-economic cost estimates, ranges are suggested with a lower bound of zero in all cases (representing no additional risk from system integration). Analysts are encouraged to use their professional judgment in selecting a value appropriate for the context and degree of conservatism sought in the FOAK cost estimate.

\subsubsection{Other FOAK Cost Factors}

Two additional capital cost factors for pre-commercial technologies are highlighted based on contractor experience [47]: cost escalations during construction, and the cost and scheduling impacts of required permits.

Escalation of capital costs during project planning and construction is a standard factor in cost estimation (see Figure 1-1), but FOAK projects are susceptible to greater escalation since project EPC schedules are typically longer than for NOAK projects. Thus, incorporation of a real cost escalation rate is recommended for any project with more than a six-month duration to provide additional confidence in the overall cost estimate [47]. Numerical assumptions would depend strongly on economic conditions in the region and industry of the study, and should be informed by current information on price trends for relevant materials and equipment.

Cost and schedule requirements for obtaining necessary permits to operate a FOAK facility are often overlooked in the initial stages of advanced technology development. While permitting costs also are a standard element of bottom-up cost estimates [16], pre-commercial technologies typically require longer schedules and higher costs than mature technologies. This would also apply to certain new classes of permits, such as a Class 6 underground injection well for storage of $\mathrm{CO}_{2}$ from a CCS system, Thus, a prudent step in cost estimation is to prepare a table of all necessary permits required for plant operation, as illustrated by the "permitting matrix" in Table 1-6 (with hypothetical entries for several common permit types). Often, a third-party specialist or consultant is engaged to prepare this information once a project location is specified [47].

Table 1-6. Illustrative example of a project permitting matrix (actual entries may vary) [47]

\begin{tabular}{|c|c|c|c|c|}
\hline Regulation & $\begin{array}{c}\text { Regulatory } \\
\text { Agency }\end{array}$ & $\begin{array}{l}\text { Permit Submittal } \\
\text { Format }\end{array}$ & $\begin{array}{l}\text { Permitting } \\
\text { Timeframe }\end{array}$ & Risks \\
\hline Building Permit & $\begin{array}{l}\text { 'Smith County' } \\
\text { Planning Dept. }\end{array}$ & $\begin{array}{l}\text { Submittal and } \\
\text { approval of } \\
\text { construction plans }\end{array}$ & $10-20$ days & $\begin{array}{l}\text { Low Risk - Common application } \\
\text { and current projects indicate } \\
\text { standard processing time }\end{array}$ \\
\hline $\begin{array}{l}\text { Clean Water Act } \\
\text { Section } 404\end{array}$ & $\begin{array}{l}\text { U.S. Army } \\
\text { Corps of } \\
\text { Engineers }\end{array}$ & $\begin{array}{l}\text { Individual or } \\
\text { nationwide permit }\end{array}$ & 6-12 months & $\begin{array}{l}\text { Low Risk - Common application; } \\
\text { can be mitigated by avoiding } \\
\text { existing wetland areas }\end{array}$ \\
\hline $\begin{array}{l}\text { 'Smith County' } \\
\text { Planning/Zoning } \\
\text { Approval }\end{array}$ & $\begin{array}{l}\text { 'Smith County' } \\
\text { Planning } \\
\text { Development }\end{array}$ & $\begin{array}{l}\text { Conditional use } \\
\text { permit }\end{array}$ & $\begin{array}{l}\text { Estimated at 6- } \\
12 \text { months }\end{array}$ & $\begin{array}{l}\text { High Risk - No project work can } \\
\text { begin without approved } \\
\text { Conditional Use Permit }\end{array}$ \\
\hline $\begin{array}{l}\text { Other Local, State or } \\
\text { Federal requirement }\end{array}$ & Agency Name & $\begin{array}{l}\text { Permit submittal } \\
\text { format }\end{array}$ & $\begin{array}{l}\text { Estimated time } \\
\text { for approval }\end{array}$ & $\begin{array}{l}\text { Estimated risks to project } \\
\text { schedule }\end{array}$ \\
\hline
\end{tabular}

\subsubsection{O\&M cost factors}

Details of O\&M cost categories and estimation methods are illustrated in a recent NETL cost study [43] and other references (e.g., [6]). In general, estimated O\&M costs for FOAK technologies or projects are expected to be higher than for an equivalent NOAK installation. The sections below discuss some of the considerations in estimating the magnitude of FOAK costs. In all cases, reporting and justification of 


\section{assumptions for the fixed and variable $O \& M$ cost parameter highlighted below are imperative for a credible cost estimate.}

\subsubsection{Variable O\&M cost items}

Analogous to an LCOE calculation for a power plant, this calculation would combine the estimated capital costs (for buildings and equipment), O\&M costs (for feedstock materials, labour, utilities, etc.), and financing cost to estimate a cost (or price) per unit of product at the production scale needed for the FOAK facility. Such an estimate, if feasible, would provide a reasonable bound for purposes of a FOAK cost estimate.

All other VOM costs can be estimated following the standard cost guidelines cited earlier. Note that the NETL methodology [16] considers the maintenance costs for planned and unplanned equipment outages to also be variable cost items. Here too, prudence would suggest that these estimated costs also will be higher for FOAK installations where reliability data are lacking.

\subsubsection{Fixed O\&M Cost Items}

Fixed O\&M (FOM) costs are typically higher for FOAK facilities because of the likely needs for increased maintenance labour, operating labour, and engineering to support the operation of a new technology. Estimates of these requirements should be developed and incorporated into the FOAK cost estimate. Labour productivity assumptions also are critical, as many project cost overruns have been attributed to worse than predicted productivity. All other FOM costs can be estimated following the NETL guidelines cited earlier. Several of these cost items also are likely to be higher for FOAK facilities than NOAK projects (see Table 1-7). In these cases, adjustments to NETL guideline values for current commercial technologies should be made based on the nature, complexity, and current maturity of the advanced technology being incorporated into the FOAK facility.

Table 1-7. Examples of increased fixed O\&M costs expected for FOAK projects (Based on [47])

\begin{tabular}{|l|l|}
\hline Fixed Cost Item & \multicolumn{1}{c|}{ Reason for Higher FOAK Cost Relative to NOAK } \\
\hline Regulatory Fees & $\begin{array}{l}\text { A FOAK facility operating new technology is expected to have higher regulatory cost } \\
\text { exposure than an NOAK facility. }\end{array}$ \\
\hline $\begin{array}{l}\text { Professional Services } \\
\text { (accounting, legal, etc.) }\end{array}$ & $\begin{array}{l}\text { Legal support in the form of supplier warranty management, claims management, and } \\
\text { overall commercial risk mitigation is expected to be greater for a FOAK facility and the } \\
\text { primary contributor to higher cost. }\end{array}$ \\
\hline Tools/Equipment & $\begin{array}{l}\text { In general, new and/or novel (FOAK) technology is expected to require more special tools } \\
\text { and plant maintenance equipment. }\end{array}$ \\
\hline Lease Expenses & $\begin{array}{l}\text { In most cases, a FOAK facility is expected to operate for a shorter period of time than an } \\
\text { NOAK facility and thus pay higher land lease rates (since shorter lease occupancy periods } \\
\text { typically incur higher lease payments). }\end{array}$ \\
\hline Training & $\begin{array}{l}\text { For a unique FOAK technology, there is low likelihood of knowledge transfer of maintenance } \\
\text { technicians, plant operators, and plant engineers from other similar facilities; therefore, } \\
\text { significant initial and ongoing training is likely to be required for FOAK facility staff. }\end{array}$ \\
\hline $\begin{array}{l}\text { Property Taxes and } \\
\text { Insurance }\end{array}$ & $\begin{array}{l}\text { Due to a lack of prior commercial experience, a FOAK facility will likely incur higher } \\
\text { insurance premiums for industry-aligned coverage. }\end{array}$ \\
\hline
\end{tabular}

\subsubsection{Financing and plant utilization factors}

As discussed earlier in Section 1.3.3, financing and plant utilization factors are used together with capital and O\&M costs to calculate the levelised cost of electricity (LCOE) for a power plant. However, the numerical values of financial and plant operating variables assumed for FOAK plants will differ from those for mature NOAK plants. In general, FOAK values for these parameters reflect the higher level of 
risk associated with first-of-a-kind projects. This, in turn, increases the LCOE relative to NOAK assumptions.

The key financial factors that distinguish FOAK projects from NOAK facilities are the weighted cost of capital and the expected lifetime (book life) of the project. These are the principal determinants of the fixed charge rate (FCR) used to amortize the capital cost of a project (see NETL [16] for details). Because FOAK projects lack prior operating experience, they typically incur a risk premium in the form of a higher cost of capital for project financing. Past NETL studies, for example, assumed a "high risk" weighted cost of capital that was 10\% (0.7 percentage points) higher than the "low risk" (also called "commercial") cost of capital [10]. Others, however, assume much larger differences (e.g., several percentage points) between high-risk and low-risk projects. In general, however, the magnitude of the risk premium and cost of capital for a FOAK facility will depend on the circumstances of the particular project and the risk profile of the pre-commercial technology. Technologies that are still in the earlier stages of development would be expected to have higher risk premiums for a commercial-scale installation than those that are closer to commercial deployment.

Some FOAK projects also are planned to have a shorter operating life than a conventional new facility (e.g., an advanced technology retrofitted on an existing power plant with a 20 -year remaining life). For a given cost of capital, a shorter project lifetime produces a higher capital charge rate, resulting in a further increase in the estimated LCOE. Therefore, the assumed project lifetime should be carefully considered and reported in any FOAK cost study.

Finally, the levelised capacity factor over the life of the FOAK plant also is likely to differ significantly from the value assumed for an NOAK facility. Two general factors contribute to this difference. One is the perspective or purpose of the cost analysis (i.e., what if vs. what will). The other is the technical readiness and reliability of the advanced technology being costed.

A bottom-up FOAK analysis to address a Type 2 (What Will) question should employ the most realistic capacity factor for the pre-commercial technology under study. This is in contrast to the bottomup NOAK analysis discussed in Section 1.3, which was motivated by a Type 1 (What If) question for a hypothetical facility meeting specified criteria and assumptions that may or may not be achievableincluding assumptions about CF.

Section 1.3.3.2 earlier presented illustrative data on the recent historical values of capacity factor for U.S. fossil fuel power plants. Such data can serve as a benchmark and guide for FOAK facilities resembling current plants. Historical data also are available from the EIA for other plant types. In some cases, estimates of future load factors may be available from detailed capacity planning models for a particular region. For new plants, it should be kept in mind that the first few years of operation typically have lower utilization levels during the break-in period, which has a significant impact on the levelised value due to the effect of discounting [8]. This is especially important for FOAK facilities because of their characteristically slower ramp-up rates as bugs are ironed out over the initial years of operation. In all cases, the choice of levelised capacity factor also should be based on a consideration of how the facility under study is expected to operate (e.g., baseload vs. cycling or peaking), along with the frequency and duration of planned outages.

Capacity factor assumptions also should take into consideration the likelihood that an advanced technology being built and operated at a commercial scale for the first time will have several years of higher forced outage rates than a commercial technology [47]. This is likely to further reduce plant capacity factors during the initial years of operation, which weigh most heavily in levelised value 
calculations. ${ }^{5}$ Thus, the levelised capacity factor for FOAK cost estimates should be less than that of a similar commercial facility.

\subsubsection{Towards a future NOAK plant}

The capital and O\&M cost estimates for a FOAK installation discussed above provide the starting points for estimates of the actual expected cost of a future mature plant if the advanced technology is successfully commercialized and adopted broadly. Additional assumptions about the future financial and plant utilization factors of an NOAK facility contribute to a projection of expected LCOE. Section 1.5 below elaborates on the guidance for such cost projections.

\subsection{Guidelines for NOAK cost for Type 2 (What Will) questions}

This section of the chapter elaborates on guidelines for conducting the type of hybrid cost analysis discussed in Section 1.2.3 and depicted graphically in Figure 1-8. Beginning with the current (FOAK) cost of an advanced technology, empirically-based learning rates are applied to estimate its future cost assuming it is successfully commercialized and widely adopted.

Methodologically, these guidelines adopt the basic procedures outlined in a 2014 NETL report [23], which drew heavily on work published by the International Energy Agency Greenhouse Gas R\&D Programme $[26,56]$. Those guidelines noted that many advanced technologies of interest are single process components that are integrated into an otherwise mature system, such as a commercial power plant. For more complex advanced technologies, a new methodological feature is the additional system contingency cost (Table 1-5) that is ascribed to the overall plant rather than a particular sub-section.

The overall cost of the system (e.g., the LCOE) is also an important metric of interest, with additional parameters whose values may change with experience. Accordingly, there are five basic steps in estimating future component and plant costs using experience curves:

- Step 1: Decompose the overall plant into major technology sub-sections.

- Step 2: Estimate the current overall plant cost and sub-section contributions.

- Step 3: Select appropriate learning rates for each major sub-section.

- Step 4: Set the starting point for learning and end point for future cost estimates.

- Step 5: Compute future sub-section and system contingency costs as a function of cumulative experience and aggregate for plant-level results.

Each of these steps is discussed in the sections below.

\subsubsection{Decomposing a plant into sub-sections}

While the principal focus of this chapter is the future cost of an advanced technology (such as a novel $\mathrm{CO}_{2}$ capture process), the most important cost metric for decision-making is often the cost of the overall plant or facility employing that technology. Frequently, the remaining components of such plants are commercial and widely-used systems, such as a conventional PC or NGCC power plant. Thus, the first step in a plant-level analysis using experience curves is to decompose the overall facility into sub-sections whose costs are likely to change at different rates for a given increment of new plant capacity.

For the case depicted above, where a new process or component is added to an otherwise mature plant design, two sub-sections may suffice: the advanced technology and the balance of the plant. In other cases, additional decomposition may be desired or helpful to refine the analysis. The principal criterion for the choice of sub-sections is their level of technological maturity and operating experience, as reflected by their cumulative installed capacity (the primary variable for experience curves, as discussed

\footnotetext{
${ }^{5}$ In some cases, a plant may be able to continue operating without the advanced technology, such as a post-combustion CCS system that can be bypassed. In such cases, however, an adjustment must be made for the additional emissions that would occur while the capture plant is offline.
} 
in Section 1.2.2.1). This is because a big percentage increase in the total capacity of a new technology (with a significant reduction in cost) typically corresponds to a relatively small change in the cumulative capacity of a mature technology that is already widely deployed. The result is a correspondingly small (often negligible) change in the cost of mature components, no matter what their learning rates (see Table $1-4)$.

In cases where there are significant differences in the current maturity of technological components, another consideration in the choice of plant sub-sections is the availability of appropriate learning rate data for the different technologies. Where such data are available (see Section 1.2.2.2), additional subsections may be warranted to refine the analysis. Table C-1, in Appendix C, illustrates the choice of plant sub-sections for four types of fossil fuel power plants equipped with new technologies for $\mathrm{CO}_{2}$ capture.

\subsubsection{Estimating current sub-section costs}

Section 1.4 provided guidelines on estimating the current cost of a full-scale commercial facility that may include both advanced and mature technology components. For purposes of future cost projections for power plants two cost measures are needed: the total capital requirement per unit of net plant capacity (typically $\$ / \mathrm{kW}$ ) and the total annual $O \& M$ cost of the facility per unit of electricity generated (typically $\mathbf{\$} / \mathbf{M W h}$ ). For other industrial processes, these costs would be normalized on the capacity and unit cost of the major product. FOAK values of financing and plant utilization factors serve as reference points for cost projections but are not typically subject to continuous experience curves; rather, new values of these parameters are assigned periodically as a technology matures, as discussed in Section 1.5 .6 below.

As a starting point for future cost projections, the total FOAK capital cost of the plant should be decomposed to obtain the cost of each sub-section identified in Section 1.5.1. This exercise is usually straightforward since the plant-level capital cost is built up from the estimated costs of all plant components, as illustrated earlier in Table 1-4.

Similarly, fixed and variable $O \& M$ costs also should be allocated to plant sub-sections. This too is a straightforward procedure employing the component-level data used for FOAK cost estimates (see Section 1.4.3). Note that the cost of energy in the form of electricity and/or steam used to operate subsection equipment is not considered to be a variable O\&M cost item at the sub-section level if that energy is generated within the plant and not purchased from an external supplier (as is typically the case for integrated plant designs). ${ }^{6}$ Rather, the requirements for electricity and steam are reflected in the net plant efficiency and the resulting plant-level requirement for purchased fuels such as natural gas or coal. Thus, the total fuel energy requirement per unit of net plant output is tracked to account for all in-plant energy use. A learning rate for this parameter then reflects any improvement in the overall plant efficiency as advanced technologies or other plant components mature.

\subsubsection{Selecting sub-section learning rates}

The choice of a learning rate for each plant sub-section is based on a judgment as to what technology or historical learning rate (or range of rates) offers the best match or proxy for the plant area in question. It also depends on the purpose or objective of the analysis and the questions or decisions it is intended to inform.

Section 1.2.2.2 summarized the learning rates reported in recent studies and literature reviews for power plant and related chemical process technologies. In general, the experience curve literature is focused mainly on capital costs; relatively few studies report learning rates for annual O\&M costs. Overall,

\footnotetext{
${ }^{6}$ For purposes of estimating the total cost of an individual plant component, some cost studies do include a cost for energy drawn from within the plant, treating it as if it were a purchased commodity. However, such cost assumptions are arbitrary since there is no unique way to ascribe a unit price (e.g., $\$ / \mathrm{MWh}$ or $\$ / 10^{6} \mathrm{Btu}$ ) to electricity and steam generated within a power plant [56].
} 
reported learning rates span a large range, even among different studies of a given technology. So how is one to choose an appropriate value (or values) for a particular study?

The most straightforward choice of a learning rate for a sub-section is the reported value for a technology that is identical or similar to one under study. For example, decomposing a coal-fired or gas-fired power plant will yield some sub-sections that directly correspond to commercial technologies with reported learning rates in Section 1.2.2.2. In the case of an advanced technology with no historical data, prospective learning rates based on modelling studies may be available or must be estimated on some other basis. For instance, advanced process designs may have attributes that resemble those of other current technologies (such as physical similarities, reactions, or physical/chemical processes). For example, a study to estimate the future cost of power plants with CCS [26] argued that a post-combustion amine-based system for $\mathrm{CO}_{2}$ capture closely resembled the basic design of a post-combustion wet limestone flue gas desulfurization (FGD) system for $\mathrm{SO}_{2}$ capture. Thus, learning rates for FGD systems were used as a proxy for $\mathrm{CO}_{2}$ capture technology (see Table C-2 in Appendix C). Other examples of learning rate assumptions based on analogies can be found in the literature (e.g., [57]).

In cases where an advanced technology has no suitable retrospective or prospective analogues to serve as a proxy for learning rates, two other options are available. One is to rely on expert judgments, as in the hierarchy also proposed by Thomassen et al. [29]. Table 1-2 includes examples of such estimates. The other option draws on some general characteristics of historical learning rate data for guidance. The first is that the highest learning rates (e.g., 20-30 percent) are typically associated with smaller-scale technologies that are modular in nature and amenable to mass production, such as solar cells and panels. In contrast, learning rates are significantly lower for large-scale process systems and technologies that are typically field-erected and designed for a unique site or size (such as power plant boilers, steam turbines, chemical processes, and air pollution control systems). Such technologies typically have learning rates on the order of 10-15 percent. Thus, the type of technology under study can make a significant difference (a factor of two or more) in the value of an appropriate learning rate. In general, the lowest learning rates (on the order of 1-5 percent) are found for mature fossil fuel power plants using commercial technologies (see Section 1.2.2.2).

Another characteristic of the historical learning rate data is the significant variability often seen in the results across studies by different researchers using different data sources from different locations and different time periods. Because a variety of factors contribute to such results, the prevalent one-factor learning model can only approximate the expected outcome for a particular technology. Thus, the average learning rate from multiple studies is a reasonable best choice in such cases. However, a thorough uncertainty analysis is indispensable in any application of learning rates (see Section 1.6.1 below).

Finally, as noted above, the choice of a learning rate also may depend on the purpose of the analysis and the questions or decisions it is intended to inform. For example, if the objective is to obtain a conservative or upper-bound estimate of future cost, then a low value of learning rate, within a range of historical values, would be appropriate. In contrast, if the objective is to estimate a best-case or optimistic future cost, then a higher rate of learning would be appropriate. In the latter case, however, care must be taken to avoid assumptions that lie outside the range of historical experience for similar types of technology if the result is to be credible for a Type 2 (What Will) cost estimate.

\subsubsection{Starting and end points for experience curves}

As illustrated earlier in Section 1.2.3, the application of an experience curve using the hybrid method requires assumptions for when cost reductions begin and how long it continues at the specified rate. The guidelines for such assumptions depend upon the current maturity of the plant sub-section or technology in question, as explained below.

\subsubsection{Guidelines for advanced (Pre-Commercial) technologies}


Advanced technologies that have not yet been deployed at a commercial scale are the main focus of this report. For such technologies, the choice of a starting point for learning (denoted in Figure 1-8 by the point $\mathrm{C} 1$ ) depends on whether one or not the current (FOAK) cost estimate for a commercial-scale project would indeed be accurate were such a facility actually built. If so, this is the appropriate starting point for cost projections. If no other large-scale facilities have been built and operated, the size of this first commercial project would represent the cumulative installed capacity, $\mathrm{C} 1$.

However, historical experience for power plants and related technologies indicates that cost estimates, as well as actual costs, for FOAK projects or plant components often fall short of the actual cost required to achieve successful commercial operation. In many cases, this is because the process fails to perform as reliably or as well as expected. Many early FGD installations, for example, experienced corrosion problems, clogging, and poor $\mathrm{SO}_{2}$ removal rates that resulted in unplanned outages and increased maintenance costs [14]. Only after improved - and much more costly - designs for the technology were built and operated successfully did FGD costs begin to fall along an experience curve. Similar experience has been found for other FOAK technologies [26].

The implication of these findings is that there is a reasonable chance that the true cost of the first successful installation of a new commercial technology will exceed the FOAK cost estimate above, even after allowing for the recommended process and system contingency costs. Thus, a scenario approach is advised. The most optimistic case is to assume that the FOAK cost estimate for a commercial plant is indeed accurate, that the facility operates and performs as expected, and that further cost reductions are achieved for all subsequent installations of the technology.

Alternatively, a more conservative approach is to assume that several large-scale facilities must be designed, built, and operated successfully before the estimated FOAK cost and performance for the advanced technology is actually achieved. In this case, learning does not begin until the installed capacity of the advanced technology has reached some minimum level, $\mathrm{C}_{\min }(\mathrm{MW})$, a parameter of the analysis. In effect, this parameter compensates for insufficiencies in the FOAK cost estimate. A sufficiently high value of $\mathrm{C}_{\min }$ also obviates the need to explicitly incorporate a system contingency cost factor for the first several installations.

General guidance for numerical estimates of $\mathrm{C}_{\min }$ is to assume higher values for technologies that are currently at earlier stages of development and furthest from commercial demonstration. For example, in view of early experience with FGD, SCR, and other newly-deployed power plant technologies, IEAGHG [56] assumed starting points ranging from 3,000 MW to 10,000 MW for NGCC, PC, IGCC, and oxycombustion power plants equipped with carbon capture systems. Alternatively, one could assume a certain number of plants of a given size to estimate the starting point capacity. In all cases, a delay in the onset of learning results in slower reductions in cost vs. cumulative capacity.

At the far right end of the cost curve, an assumption also is needed for the end point of the experience curve projection. If the objective of the analysis is to estimate the likely NOAK cost of the advanced technology a definition is needed as to what constitutes the mature $\boldsymbol{N}^{\text {th }}$ plant. The earlier discussion in Section 1.2.3.2, together with the illustration in Figure 1-4, showed that the cost curve tends to flatten after about 15 to 20 replications of the initial capacity, based on the one-factor model for learning. For a mid-value learning rate of 15 percent, twenty replications (a little over four doublings) is also the point at which the cost falls to half its initial value. If this is taken to be the end point for the analysis of an advanced $500 \mathrm{MW}$ power plant, the cumulative installed capacity would be $10 \mathrm{GW}$ for the optimistic case where learning is assumed to begin after the first installation. If the onset of learning is delayed, the endpoint capacity to reach a 50 percent cost reduction would be greater. Other studies assume that the cost of new technologies decline at different rates. The EIA National Energy Modeling System (NEMS) model, for example, optimistically assumes that learning begins immediately after the first installation of a new technology and that learning rates decline over three periods of cumulative capacity [58]. Additional details of EIA assumptions are shown in Section 1.6.1.2. 


\subsubsection{Guidelines for commercial technologies}

Conceptually, starting points for learning (i.e., an estimate of the current installed capacity) also are required for plant sub-section technologies that are currently commercial and already in wide use. Common examples include PC and NGCC power plants to which an advanced technology, such a novel $\mathrm{CO}_{2}$ capture system, is added. In these cases, however, precise estimates of total current capacity are not usually necessary because the incremental capacity of the new advanced technology is typically small relative to the current base of established technologies. This is illustrated in Table C-3, in Appendix C, which shows several hundred GW of initial capacity for PC and NGCC plants worldwide in the IEAGHG study [56]. Thus, a relatively large (e.g., $10 \mathrm{GW}$ ) increment of advanced technology capacity represents only a small percentage change in the total capacity of mature technologies, resulting in a small (often negligible) change in cost due to learning. In addition, mature plants also tend to have lower learning rates, which further contribute to small or negligible reductions in these sub-section costs. In such cases, it can be assumed that the cost of mature plant sub-sections is unchanged by small increments of new capacity, obviating the need for an experience curve analysis. (see Section 1.5.5 for a numerical example that illustrates this statement.)

In cases where a commercial technology has not yet reached maturity in a particular application (e.g., a power plant design employing coal gasification) several factors must be considered in estimating the current capacity of the technology to be used in the learning rate equation. One is the geographical scope for learning, specifically, whether it is based on regional, national, or global installations. This is important since the broader the scope, the larger the initial capacity estimate. This, in turn, results in a smaller cost reduction for a specified increment of new capacity. The guidance for this case is that the geographic scope for current capacity estimates should reflect the scale at which learning occurs for the technology in question. In most cases, this will be based on global installations, reflecting a world in which information and experience is widely shared, and multiple vendors compete for markets, so that all users of the technology benefit from learning and cost reductions. While this may not always be the case, it is the most prevalent model for technologies whose learning rates have been studied and quantified [59].

Another factor in estimating current capacity is the so-called "spillover effect" where experience with a technology in applications different from the one under study indirectly contributes to learning and cost reductions. Examples include the use of $\mathrm{CO}_{2}$ capture systems and coal gasifiers in industrial processes as opposed to power plants. The addition of spillover effects further increases the initial capacity estimate, which again affects the magnitude of cost reduction for an additional increment of capacity. In cases where the spillover effect is sizeable, estimates should be made of the "equivalent capacity" of installations that contribute to knowledge spillover, based on an appropriate metric such as the volumetric gas flow treated. On that basis, the total equivalent capacity values shown in Table C-3, in Appendix C, were estimated (see Rubin et al. [26]). Absent such estimates, larger cost reductions for the advanced technology will be projected for a given increment of new capacity, based on the smaller initial value.

\subsubsection{Projecting future costs}

Once all sub-section learning rates and initial capacity values have been specified, Equation 1, shown earlier, is used to project future costs. The parameter, $b$, in Equation 1 is determined using Equation 4. A simple numerical example illustrates this calculation for a constant learning rate and an end-point capacity of $20 \mathrm{GW}$ of an advanced process component added to a conventional (mature) power plant. Additional parameter assumptions and calculated values for this example are as follows:

Advanced Technology Sub-Section:

Assume: Current FOAK cost $=\$ 3,000 / \mathrm{kW} ; \mathrm{LR}=0.15 ; \mathrm{C}_{\min }=1,000 \mathrm{MW} ; \mathrm{C}_{\text {final }}=20,000 \mathrm{MW}$

From Eq. $4: \quad b=\frac{-\log (1-0.15)}{\log (2)}=0.234$ 
From Eq. 1: $y=\frac{(3,000)}{(20,000 / 1,000)^{0.234}}=1488 \$ / \mathrm{kW}$ (at the end point)

Mature Technology Sub-Section:

Assume: Current (initial) cost $=\$ 1,000 / \mathrm{kW} ; \mathrm{LR}=0.05 ; \mathrm{C}_{\text {initial }}=200,000 \mathrm{MW} ; \mathrm{C}_{\text {final }}=220,000 \mathrm{MW}$

From Eq. 4: $\quad b=\frac{-\log (1-0.05)}{\log (2)}=0.0740$

From Eq. 1: $y=\frac{(1,000)}{(220,000 / 200,000)^{0.074}}=993 \$ / \mathrm{kW}$ (at the same end point).

One sees in this example that after $20 \mathrm{GW}$ of cumulative new capacity and experience the advanced technology component cost fell by $50 \%$, whereas the maturity sub-section cost fell by only $0.7 \%$ because of its greater initial capacity as well as its lower learning rate. Figure C-1, Appendix C, shows additional results of this type.

While most published learning rates apply only to capital costs, projections for O\&M costs and plant efficiency improvements follow the same procedure where learning rate data are available. At the end point of the analysis (or any intermediate point, if desired) the various sub-section costs are then summed to obtain the total capital and O\&M costs of the plant.

Similarly, a levelised cost of electricity (LCOE) calculation can be performed at any point in the future capacity projection by introducing appropriate values of levelised capacity factor and fixed charge factor. These two parameters also change as a new technology matures: capacity factor is likely to increase as plant reliability and availability improve. The weighted average cost of capital, and thus the FCF, typically declines as risks decrease with increasing experience. These changes in parameter values can be modelled simply as a step change (or a set of step changes) at a particular point(s) rather than as a continuous function. For example, one recent study assumed financing rates change from high-risk to low-risk after $10 \mathrm{GW}$ of experience with an advanced $\mathrm{CO}_{2}$ capture system [3]. This is illustrated later in the example presented in Section 1.7.

A final step that is strongly recommended in any NOAK cost projection is an uncertainty analysis for key results. This applies equally to the hypothetical cases of Type 1 (What If) cost estimates. Section 1.6 below elaborates further on methods and guidelines for characterising uncertainty in advanced technology cost estimates.

\subsection{Characterising uncertainty}

Previously published cost guidelines remind us that cost estimates commonly reported as single (deterministic) numbers are actually the central value of a range or distribution of possible outcomes (e.g., $-30 \%$ to $+50 \%$ for an AACE Class 4 cost estimate) $[16,60]$. While uncertainties are inherent in all cost estimates undertaken for technology assessments and screening (e.g., AACE Class 4 or 5, as defined in [43]), costs for advanced technologies that are not yet commercial have significantly greater uncertainty and/or variability. One recent study, for example, found that independent capital cost estimates for the same advanced carbon capture process for a coal-fired power plant differed by as much as 65 percent, mainly because of differences in equipment sising methods and sources of cost information [45].

The hybrid costing method described in this chapter introduces additional uncertainties associated with the learning curve analysis. Thus, it is important to recognize and characterize all major sources of uncertainty that affect the integrity and robustness of any advanced technology cost estimate. An uncertainty analysis also can help establish empirically-based bounds and expectations for future costs and potential cost reductions. This perspective is critical for effective R\&D planning and management of early-stage technologies.

This section briefly reviews and illustrates some of the leading methods available to characterize uncertainties. They include both quantitative and qualitative methods that are discussed more extensively 
in the literature, including sensitivity analysis, probabilistic analysis, and the use of expert judgments (e.g., $[5,61])$. Readers should consult such resources to learn more about the methods highlighted below.

\subsubsection{Sensitivity analysis}

Sensitivity analysis is the most familiar and widely-used method of uncertainty analysis. Here we distinguish between two major types of sensitivity analyses: parameter uncertainty and model uncertainty. The first refers to changes in the parameter values of a particular calculation or mathematical model. The latter refers to changes in the underlying model and its parameters or variables. Further differentiations can also be made in the types of sensitivity and uncertainty analyses (see e.g., [5]).

\subsubsection{Parameter Sensitivity}

The most common method of analysing uncertainty is a "one-at-a-time" sensitivity analysis in which the value of a single parameter in a performance or cost calculation is varied from its nominal or base case value to quantify the effect on a result of interest, with all other parameters held constant. This is effectively a measure of the "local sensitivity" around the point determined by the nominal values of all parameters. Figure C-2, and Table C-4, in Appendix C, show two examples. Figure C-2 shows the sensitivity of total capital cost for an advanced power plant design to a range of learning rates applied to each plant sub-section, then summed to obtain the high and low estimates shown for the overall plant. Table C-4 shows another example in which each of seven additional parameters of an experience curve analysis are varied, in turn, from their nominal values with all other parameters held fixed. The results reveal which parameter uncertainties have the largest impact on capital and LCOE cost results.

Appendix D shows two additional examples from a study of baseline power plant costs using detailed engineering-economic models [43]. Figure D-1 shows the sensitivity of the LCOE to changes in the assumed prices of purchased fuels. Figure D-2 shows the results of a two-way sensitivity analysis in which a "phase diagram" reveals the sensitivity of the lowest-cost plant type to changes in two independent variables (in this case, the price of natural gas and the selling price of captured $\mathrm{CO}_{2}$ for plants with CCS). Other studies show examples of plant performance sensitivity to technology design variables as well as to economic and financial variables (e.g., [62]).

In most studies, however, the choice of parameters that are varied, and results that are displayed, are based on the judgment and preferences of the authors. Seldom do studies systematically identify the most important variables affecting a particular result. Consequently, important parameters and correlations between factors may be overlooked.

The guidance in this regard is to first identify the study results or model outputs that are most important, given the purpose and objectives of the study. In the context of this report, that would typically include one or more cost measures for an advanced technology component or integrated facility (e.g., its capital cost, O\&M cost, or total levelised cost per unit of product). It may also include important performance measures, such as the net plant efficiency (or heat rate), net capacity, annual power generation (for power plants), and a rate of $\mathrm{CO}_{2}$ and/or other emissions.

Next, identify the model parameters or assumptions that have the greatest influence on those key results. This can be done by varying each uncertain parameter one at a time and then viewing the change in key results with all other parameters held constant. Figure 1-9 gives an example of such an analysis in which an engineering-economic model was used to calculate the LCOE of a power plant with an advanced $\mathrm{CO}_{2}$ capture system. The tornado diagram reveals that the LCOE is most sensitive to three of the fifteen variables shown. Assumptions about these parameters thus merit particular scrutiny and justification if the cost result is to be credible. 
Sensitivity of LCOE (€/MWh, change from a base value of 102.56)

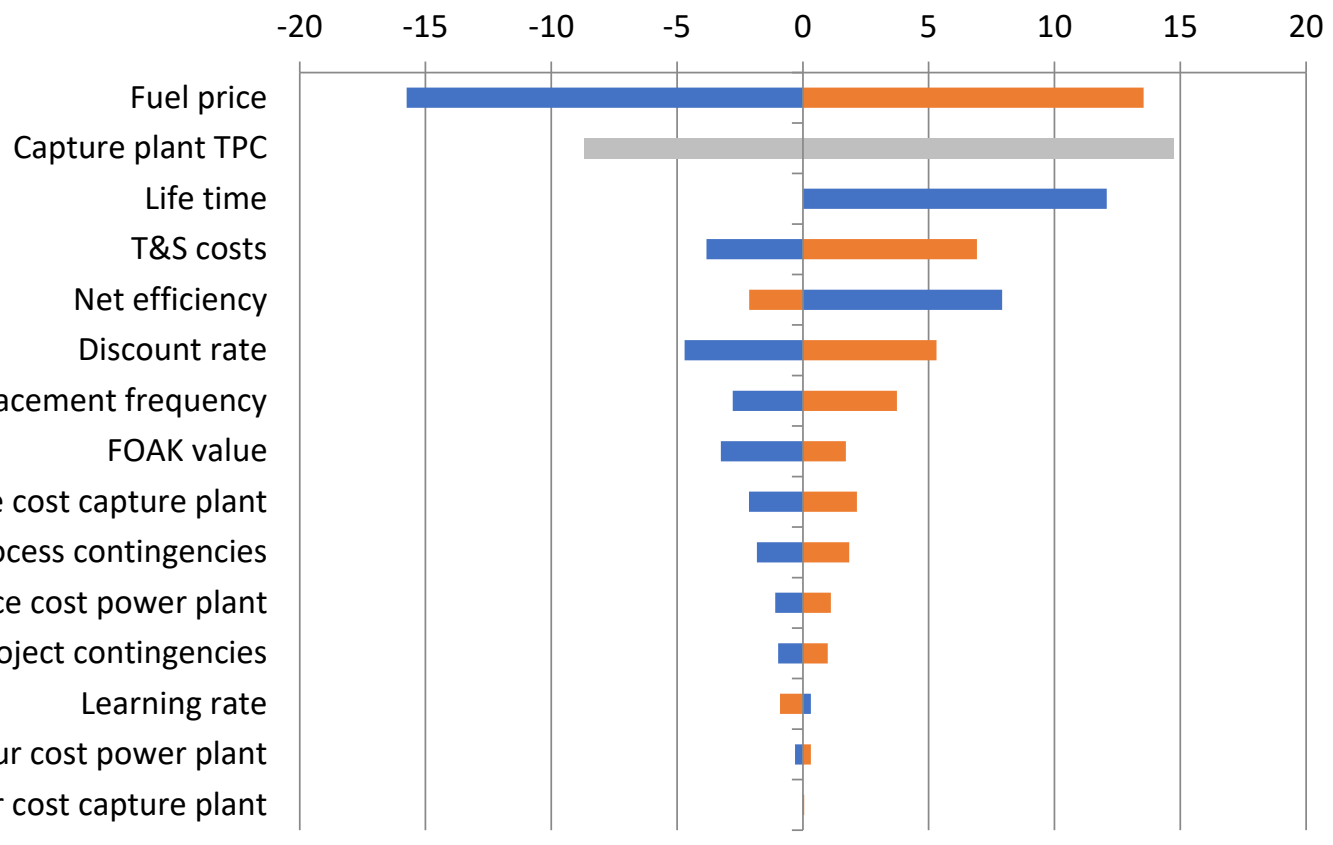

Figure 1-9. A tornado diagram indicating the change in levelised cost of electricity for a NGCC plant equipped with an advanced post-combustion $\mathrm{CO}_{2}$ capture system [57]

Ideally, a comprehensive sensitivity analysis would systematically vary the nominal value of every model parameter by a fixed percentage (e.g., plus or minus $10 \%$, subject to physical, thermodynamic, or other constraints) to first identify which variables have the greatest effect on key results. The values of parameters to which results are most sensitive should receive special scrutiny. Since some parameters may have greater uncertainty or variability than others, available data and judgments must be applied to estimate the overall uncertainty in key parameters.

In cases where a systematic sensitivity analysis is not practical (such as when using complex models with a great many variables), the judgment and experience of the analyst must be relied upon more heavily to identify the most relevant uncertain parameters. In such cases, a review of study results by an independent party can be helpful to flag any important omissions or issues. In many cases, other analysis methods may be needed to understand and characterize uncertainties more rigorously (see Section 1.6.2).

\subsubsection{Model Uncertainty}

Sensitivity analysis also can be used to characterize the effect of uncertainties in the underlying analytical models employed for techno-economic assessments, including the experience curve models discussed in Section 1.2.2.1. In addition, models themselves can be varied to assess the effect of using different mathematical descriptions for (a part of) the model. For example, while the log-linear model in Equation 1 is most commonly used for experience curves, other model formulations, such as the multifactor regression models discussed in Section 1.2.2.1, or the piecewise and nonlinear models illustrated in Figure 1-10, may better fit historical cost trend data in some situations. Thus, the data in Figure 1-10(a) are best fit by a piecewise log-linear model with an initial learning rate of 7 percent, followed by a more rapid rate of 29 percent. Similarly, the exponential models in Figure 1-10(b) indicate changing rates of learning that are believed to reflect a large initial deployment of new emission control technologies in response to new regulatory requirements, with little opportunity for learning until later generations of lower-cost systems were deployed [35]. 
(a)

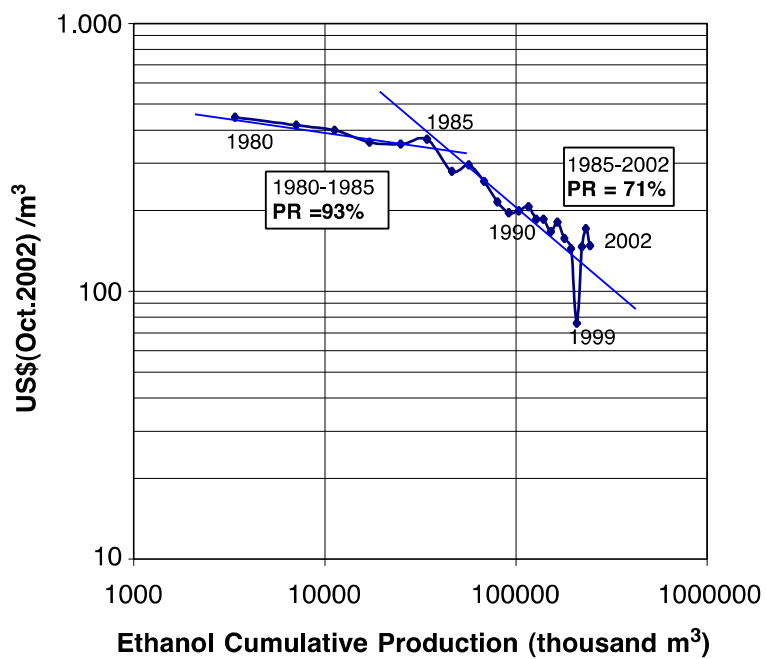

(b)

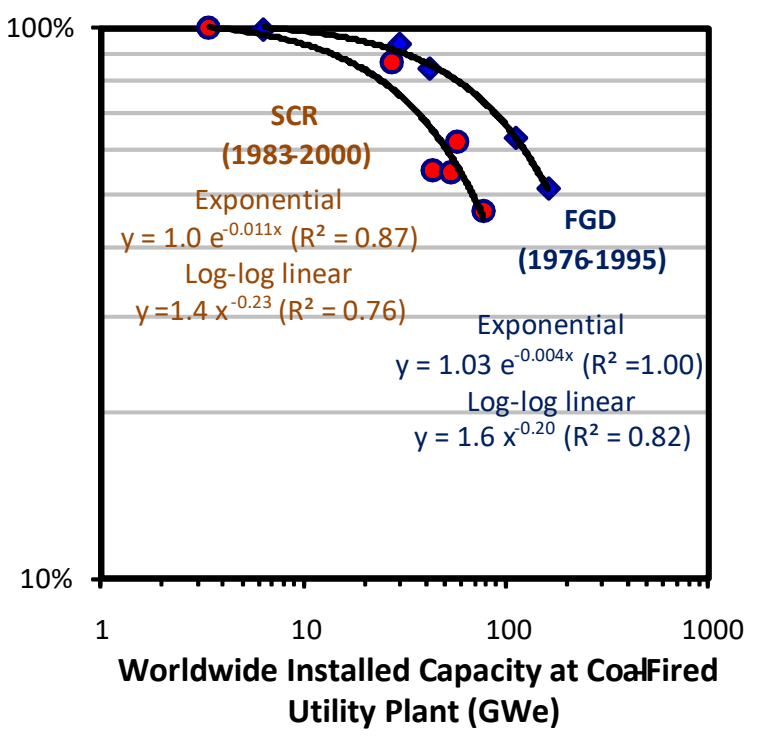

Figure 1-10. Examples of experience curves fit by (a) piecewise log-linear models with different learning rates and, (b) nonlinear exponential models [35]

A number of studies in the literature also suggest an S-shaped experience curve in which learning rates gradually decline as the technology matures and its cost approach a plateau (see Yeh and Rubin [35]). Declining learning rates for mature power plants also are assumed in the EIA NEMS model used for U.S. energy projections [58]. For designated "revolutionary" technologies (which include CCS, fuel cells, offshore wind, solar thermal, and others) an initial learning rate of 20 percent is assumed for the first three doublings of capacity, followed by an "evolutionary" stage with a learning rate of 10 percent for the next five doublings. This is followed by the "mature" stage with a learning rate of just 1 percent [59]. Figure 1-11 compares the resulting capital cost trajectory to that for three constant learning rates. One sees that after a rapid initial decline, the cost decreases more gradually; after eight doublings of the initial capacity (off-scale in this exhibit), it plateaus at approximately 30 percent of the initial cost. Table E-1, Appendix E, provides additional details of EIA learning rate assumptions for different power plant technologies.

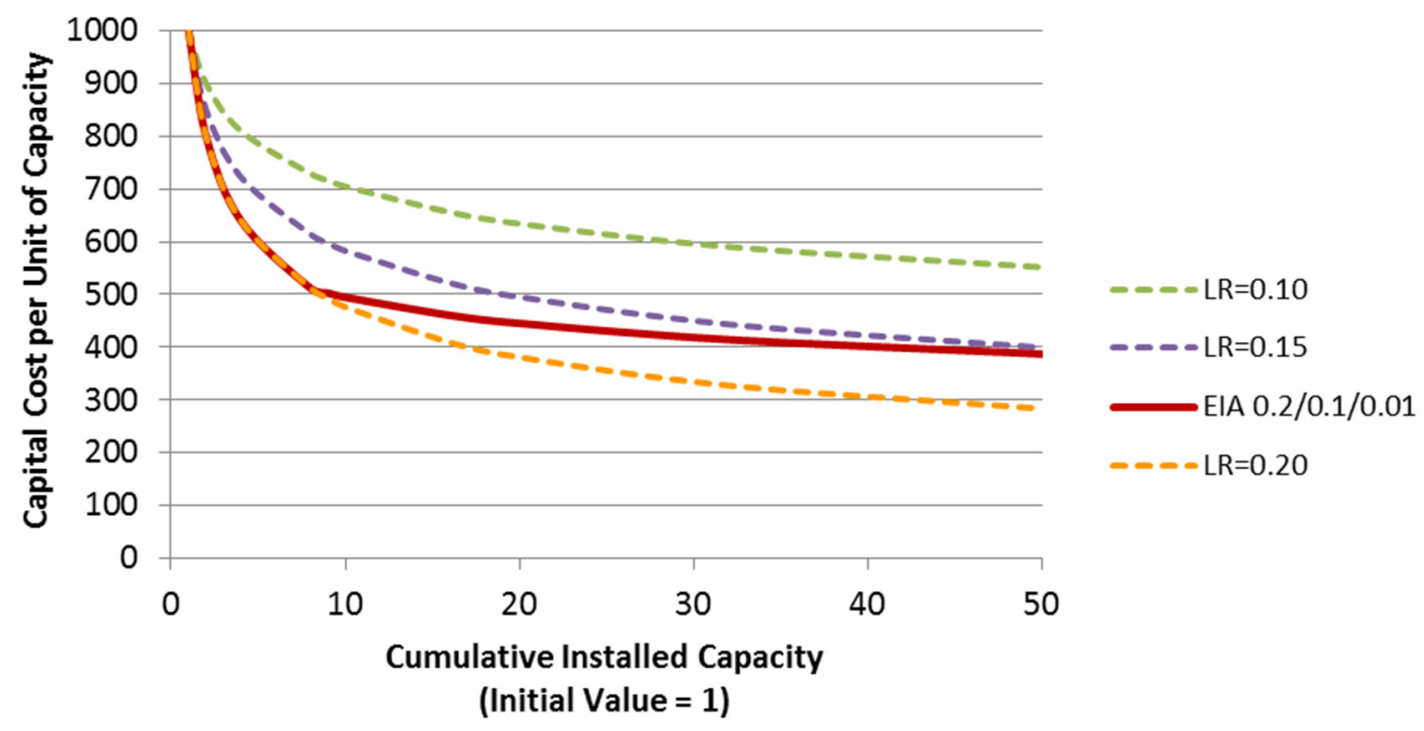

Figure 1-11. Capital cost trajectories for three constant learning rates plus the EIA/NEMS trajectory for a "revolutionary" technology with decreasing learning rates over three periods.

Yeh and Rubin [35] present a more complete discussion of learning rate uncertainties. Since technological learning is a key element of the here proposed hybrid costing method, analysts conversant with this 


\section{literature may wish to extend sensitivity studies to include alternative learning model formulations relevant to the technology of interest, in addition to the parametric variations discussed earlier.}

\subsubsection{Probabilistic analysis}

While local sensitivity analyses are useful to understand how technology cost estimates are affected by changes in key assumptions and design parameters, the large number of variables involved in many cost estimates makes it impractical to study the effect of all parameters comprehensively. More importantly, a simple one-at-a-time sensitivity analysis overlooks the combined effect of interactions among multiple parameters, each with its own uncertainty or variability. Nor does it reflect global uncertainties over the full solution space, or provide any insight into the likelihood of a particular result, which can be especially important for answering 'what will' type of questions for technology-related decision-making. A probabilistic analysis addresses many of these shortcomings.

Rather than having a single (deterministic) value, uncertain parameters are quantified as a probability distribution function (PDF) specifying the likelihood of a different value occurring. Figure 1-12 shows an example from a project cost analysis, displayed as both a PDF (red bell-shaped curve) and a cumulative probability distribution (CDF, the thin black line) [47]. From such data, one can calculate other results of interest, such as the 90 percent confidence interval shown in the figure, or other global sensitivity indicators (similar to the tornado diagram in Figure 1-9, but calculated from results of a global uncertainty analysis; see [5]).

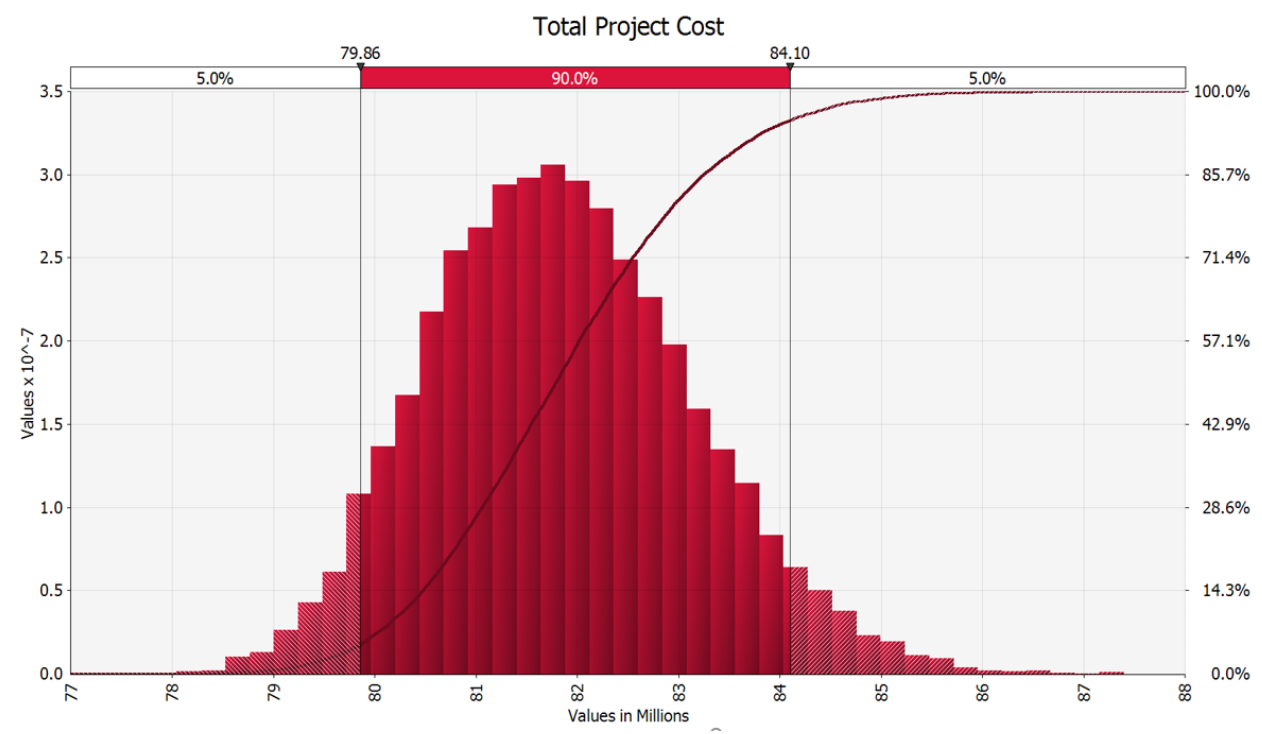

Figure 1-12. Example of a probabilistic cost distribution generated by risk estimating procedures [47]

Since few models allow direct analytical solutions of probabilistic problems, models with one or more uncertain parameters employ Monte Carlo or other efficient methods of repeated sampling of input parameter values to generate a range of numerical results that can be displayed either as a PDF or as a $\mathrm{CDF}$, such as in Figure 1-13. In that example, a detailed techno-economic model was used to calculate the hypothetical NOAK cost of a power plant employing a novel ammonia-based system for $\mathrm{CO}_{2}$ capture [63]. Probability distributions were assigned to 22 model input parameters including ten performancerelated parameters, ten cost parameters, and two financial and plant utilization parameters (see Table D-3 in Appendix D for details). Figure 1-13 shows the overall uncertainty distribution for the resulting LCOE, as well as the cumulative contributions of different parameter groups. Also shown (as a vertical line) is the deterministic base case value. Its intersection point with the cumulative probability distribution reveals a 20 percent likelihood that the true cost is less than the nominal value - thus, an 80 percent chance it 
exceeds the nominal value based on the given parameter uncertainties (Figure D-1). These results show skewed distributions for all parameter groupings, indicating a high probability that the LCOE will exceed the deterministic estimate.

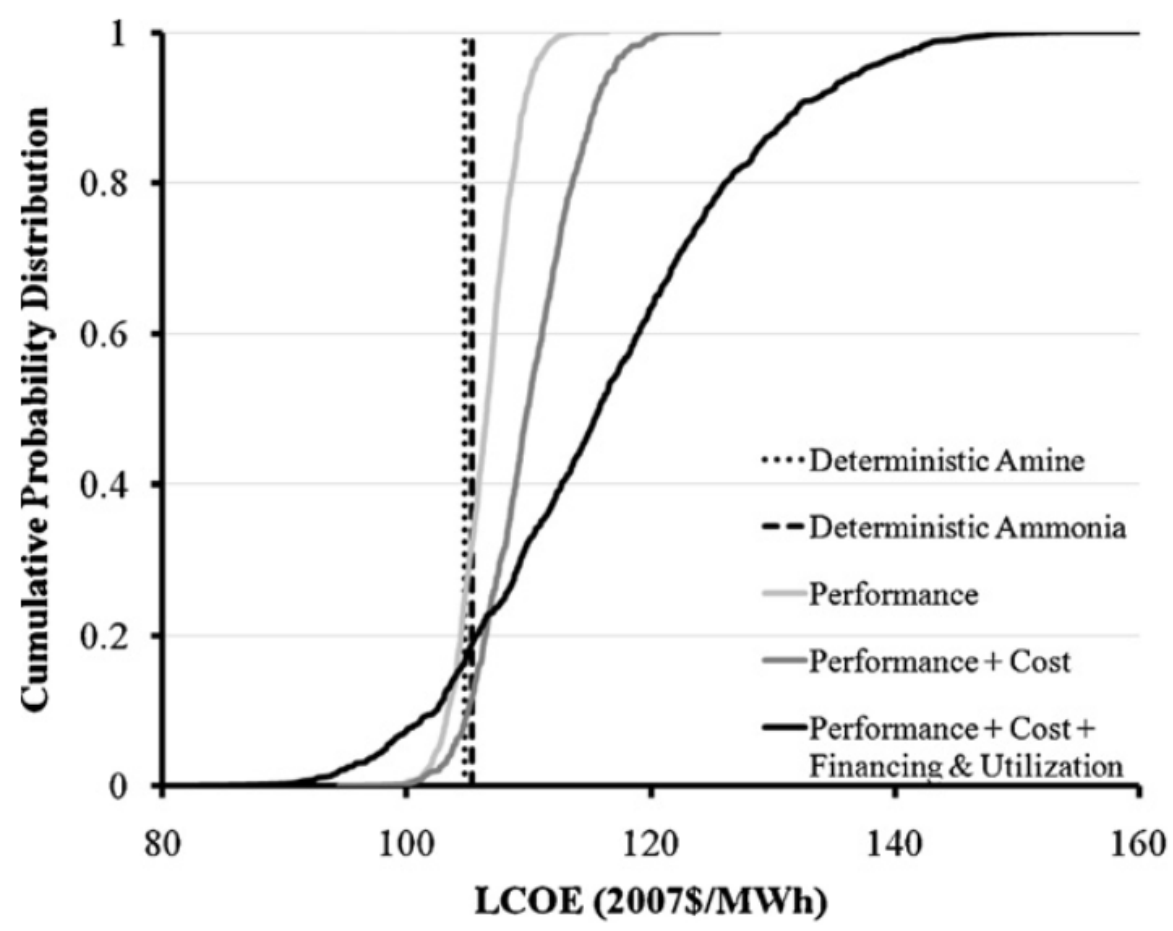

Figure 1-13. Cumulative probability distribution of LCOE for a PC plant with an advanced post-combustion $\mathrm{CO}_{2} \mathrm{Capture}$ system. The figure shows the contribution of different parameter groups to the overall uncertainty [63]

Results such as this yield quantitative estimates of the cost and performance risks, as well as potential benefits, of an advanced technology. A probabilistic analysis also can help identify the sources of greatest risk and the measures needed to reduce or eliminate those risks via changes in process design, system design, and plant operation. As with sensitivity analysis, however, a comprehensive analysis of all uncertain parameters may be impractical for complex models that have a very large number of variables. In such cases, a screening analysis, together with the expert judgment of the modeler, must be relied upon to select the most relevant parameters. The ability to conduct such an analysis also requires software tools that are now widely available commercially, or analytical modelling tools that incorporate a probabilistic analysis capability (e.g., [38]). It also requires a deeper understanding of the uses and limitations of probabilistic methods than can be presented here. Other readings are recommended for additional background (e.g., $[5,38])$.

A final topic for discussion is how to quantify the uncertainty or variability in a particular model parameter. Key to the quantification of uncertainty or variability is the availability of empirical data for the parameter of interest, as well as knowledge of physical or thermodynamic limits. Empirical data offer the most rigorous approach and is most applicable to parameter variability. This refers to measurements of technology characteristics and performance parameters, as well as data on the magnitude and variability of economic and financial parameters. In other cases, a value for true uncertainty may be set by physical or thermodynamic limits on the maximum or minimum value of a parameter used in a technology performance and cost estimate (e.g., a minimum work value for a chemical separation process). Lastly, the use of expert judgments is often required for parameter and uncertainty estimates where empirical data are lacking, or for estimating the future values of key parameters. The following section elaborates briefly on the latter topic. 


\subsubsection{Expert judgments}

In the absence of empirical data, cost and uncertainty estimates for advanced technologies rely heavily on the use of expert judgments, both for Type 1 (What If) and Type 2 (What Will) studies. Methods and protocols for expert elicitation have been widely employed to estimate various types of uncertainties when data are lacking. In the context of this report, two major questions of interest are: what values can be usefully obtained via expert elicitations, and how exactly are those values obtained?

Expert judgments are commonly used in FOAK cost studies to estimate the current cost of an advanced technology component or a material that has not yet been fabricated and produced commercially. They also are used in What-If studies to obtain quantitative estimates of the expected future cost of a current or advanced technology, often for the purpose of assessing the potential economic and/or environmental benefits of an R\&D program in a particular time frame [64]. Experts also are asked to quantify the likely future value and uncertainty of key technical and economic parameters used to calculate future technology costs. Figure 1-14 shows an example from such an elicitation used to obtain parameter values and uncertainty distributions for an engineering-economic model used to calculate the performance and cost of an amine-based $\mathrm{CO}_{2}$ capture technology [65]. More recently, expert judgments provided a set of qualitative indicators for a "pedigree analysis" to assess the strength and quality of data inputs for advanced technology cost estimates $[5,66]$.

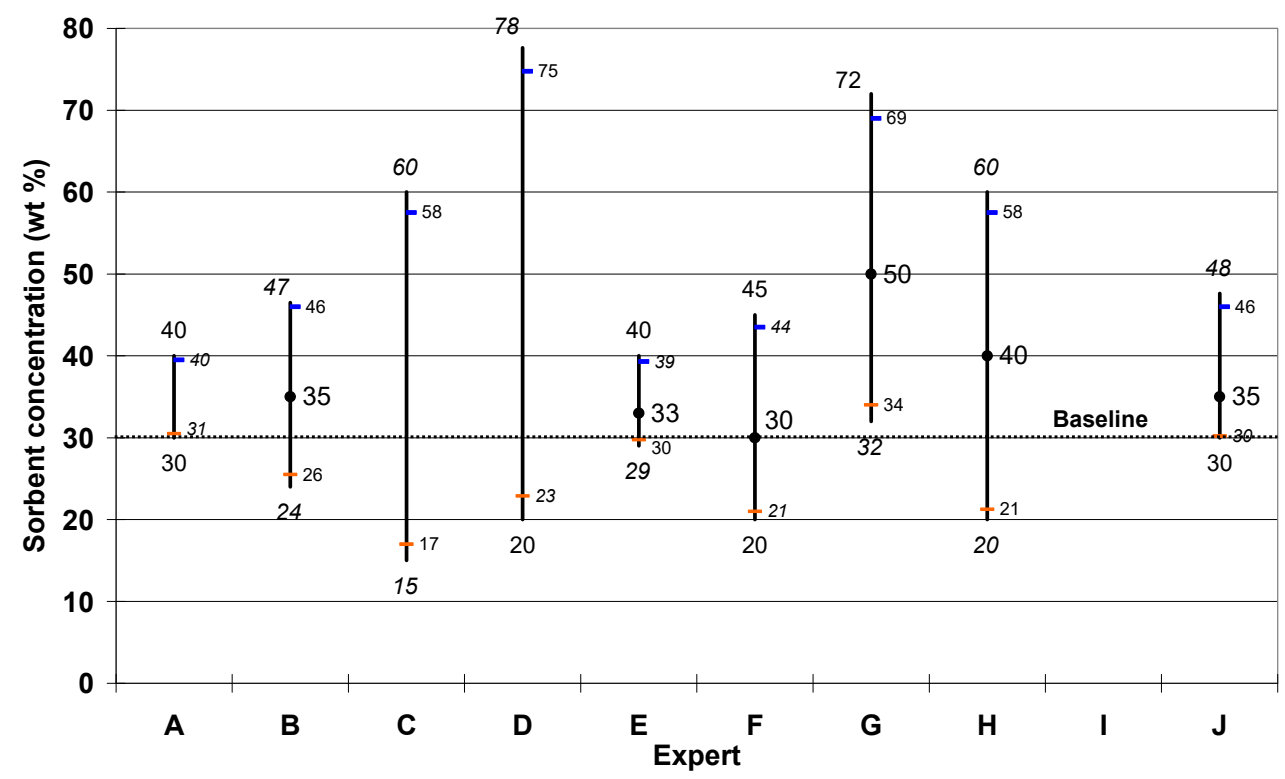

Figure 1-14. Elicited judgments from ten experts for the sorbent concentration in commercial amine systems ten years in the future assuming modest growth in R\&D. Each distribution shows the nominal value (dot), range (vertical line), and 90 percent confidence interval (small cross lines) [65]

Methods to obtain expert judgments vary widely. The most rigorous method of expert elicitation follows a detailed protocol requiring significant time and effort in the selection of experts, the preparation of appropriate background materials and questions, and the elicitation of subjective probability distributions from in-person interviews with each expert [67]. Other studies have employed written correspondence supplemented by personal and/or telephone interviews. The data in Figure 1-13, for example, were elicited from a set of experts in five different countries working in industry, academia, research organizations, and consultancies. Care was taken to formulate questions in a manner informed by expert elicitation research. Other cost studies, however, employ more informal, less structured methods of eliciting expert judgments, such as drawing upon co-workers in an organization. Depending upon its purpose and scope, the results of such elicitations may be viewed as less rigorous than a more encompassing analysis. Thus, analysts employing expert judgments in advanced technology cost estimates should be well-grounded in elicitation methods and pitfalls (e.g., as discussed in Morgan [67]). 


\subsection{Illustrative example of a hybrid cost model analysis}

To illustrate how the proposed hybrid method might be used to assess the expected future cost of an advanced technology, a simple example of a hybrid cost model analysis is presented based on a study of a new $550 \mathrm{MW}$ coal-fired power plant with post-combustion $\mathrm{CO}_{2}$ capture using an advanced polymeric membrane system with sweep gas recycle of captured $\mathrm{CO}_{2}$ [3]. The advanced plant design was taken from an earlier study [10] that used engineering-economic models to estimate the NOAK cost goal of the plant assuming that the bare erected cost of the membrane-based $\mathrm{CO}_{2}$ capture module achieves a cost goal equivalent to $\$ 80 / \mathrm{m}^{2}$ of membrane material (in 2007 dollars). The remainder of the supercritical power plant and its emission control systems were assumed to be mature commercial technologies with known costs. This study represents a typical Type 1 (What If) cost analysis.

The objective of the hybrid cost model analysis was to answer typical Type 2 (What Will) questions: Based on its current state of development, what will the actual (projected) NOAK cost of this advanced technology be? How does that compare to the NOAK cost goal in the NETL study? Based on historical experience with technology cost reductions, how many plants (with how much installed capacity of the new carbon capture technology) would it take before the NOAK cost goal could be met? What is the uncertainty in these estimates for the optimistic case where the first full-scale installation of the advanced technology performs as expected? What are the major sources of uncertainty in this analysis?

As noted earlier in this chapter, an analysis to address these questions can be undertaken at various levels of detail. The approach in this example was to expend a minimum level of effort and resources to obtain preliminary results that could be refined later if initial findings warranted a greater effort. Accordingly, a number of simplifying assumptions were made, as outlined below.

First, in the absence of reported data on the actual current cost of the novel membrane material described in the NETL [10] study, the FOAK cost estimate for the hybrid model study assumed the NETL "nearterm" capital cost goal equivalent to $\$ 150 / \mathrm{m}^{2}$ of membrane material as a lower (optimistic) estimate. A higher (more conservative) capital cost of $\$ 250 / \mathrm{m}^{2}$ was assumed as a sensitivity analysis for this preliminary assessment. The latter value assumes that production scale economies for a full-scale (550 MW) FOAK plant can achieve a cost well below the current cost range of approximately $\$ 500 / \mathrm{m}^{2}$ to $\$ 750 / \mathrm{m}^{2}$ reported for smaller-scale industrial membrane systems [68]. Based on the current level of development for the advanced membrane capture system reported by NETL [10], the process contingency cost factor was set to 35 percent, compared to 20 percent in the NETL study. All other plant costs were kept at the NETL study values.

For O\&M costs, the membrane replacement material for the FOAK plant was assumed to have a higher unit cost (proportional to its capital cost) and a $20 \%$ shorter lifetime relative to the NOAK assumptions. For calculating the LCOE, the capital charge factor was set at NETL's high-risk value for the first $10 \mathrm{GW}$ of installed capacity (about 20 plants), then reduced to the commercial (low-risk) value for subsequent projects. In the absence of historical data for membrane-based learning rates, the learning rates for capital and O\&M costs of the post-combustion membrane-based system for $\mathrm{CO}_{2}$ capture were based on those for post-combustion capture of $\mathrm{SO}_{2}$ (Table 1-1). For this analysis, learning was assumed to begin after the first installation of the advanced system, consistent with the study objectives noted earlier. Learning rates for the remainder of the supercritical PC plant were assumed to be small, with a negligible reduction in balance-of-plant cost over the projection period of $40 \mathrm{GW}$ of new capacity for the advanced technology (see illustrative calculation in Section 1.5.5).

Figure 1-15 shows the results for total capital requirement of the overall power plant as a function of increasing deployment of the advanced technology for the two estimates of FOAK cost. The NETL NOAK cost goal, called Case 5A, is shown as a horizontal dotted line. The graph shows that achieving that goal for the lower FOAK cost would require about $20 \mathrm{GW}$ of installed capacity (approximately 40 installations) of commercial experience with the advanced membrane technology. For the higher FOAK cost estimate about $80 \mathrm{GW}$ of cumulative capacity (off-scale in Figure 1-15) would be required. 


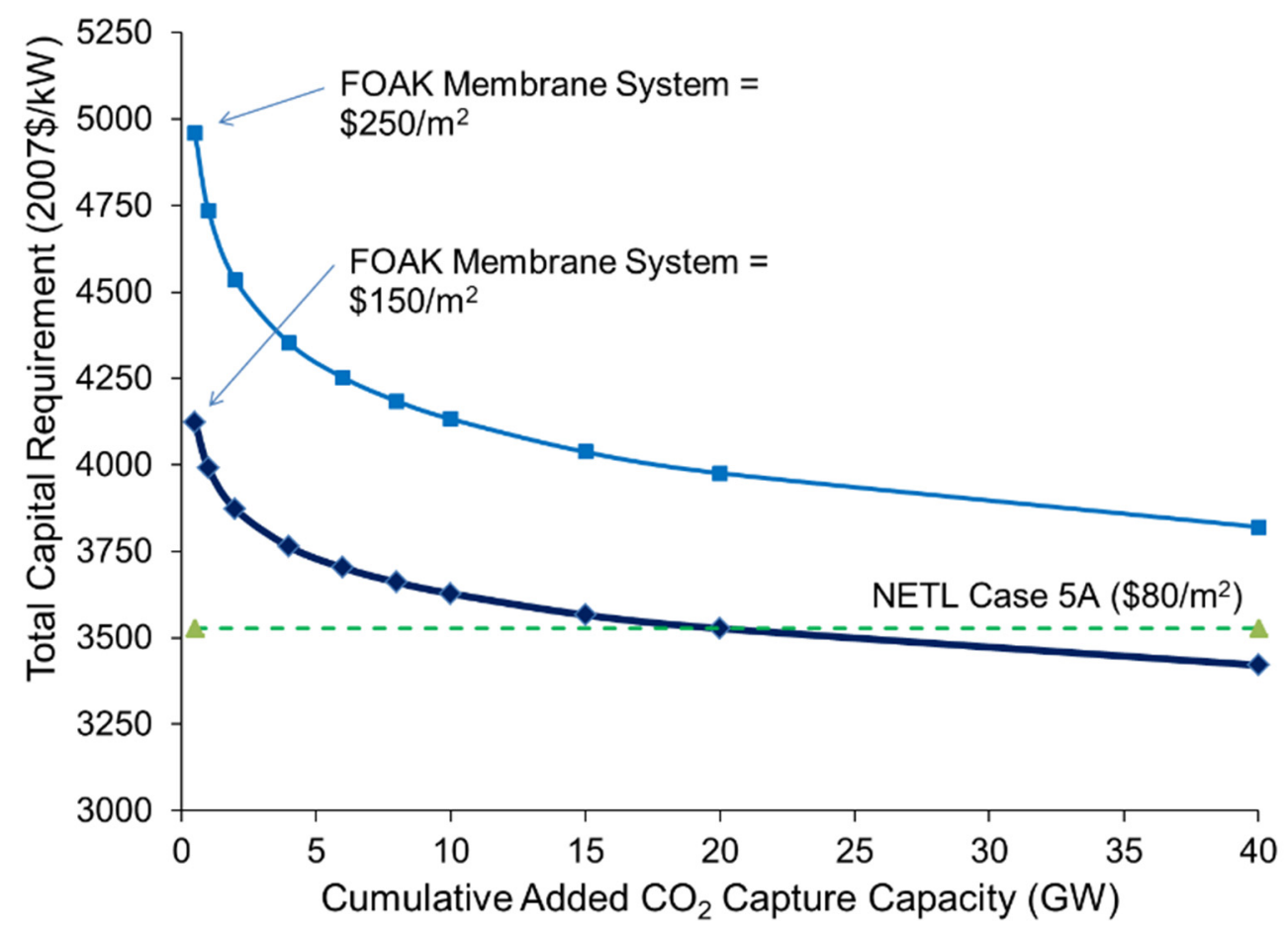

Figure 1-15. Total power plant capital requirement for the high and low FOAK cost estimates of the advanced membrane-based $\mathrm{CO}_{2}$ capture system. Also shown is NOAK cost (NETL Case 5A) meeting the membrane system cost goal of $\$ 80 / \mathrm{m}^{2}[3]$

The cost trajectory for total annual O\&M cost of the plant (not shown) follows a qualitatively similar path as in Figure 1-15, albeit with a higher learning rate for the advanced capture system, again based on FGD experience (Table 1-1). Figure 1-16 then combines all capital and O\&M costs with additional assumptions for plant financing and utilization to show the constant-dollar levelised cost of electricity for the two cases.

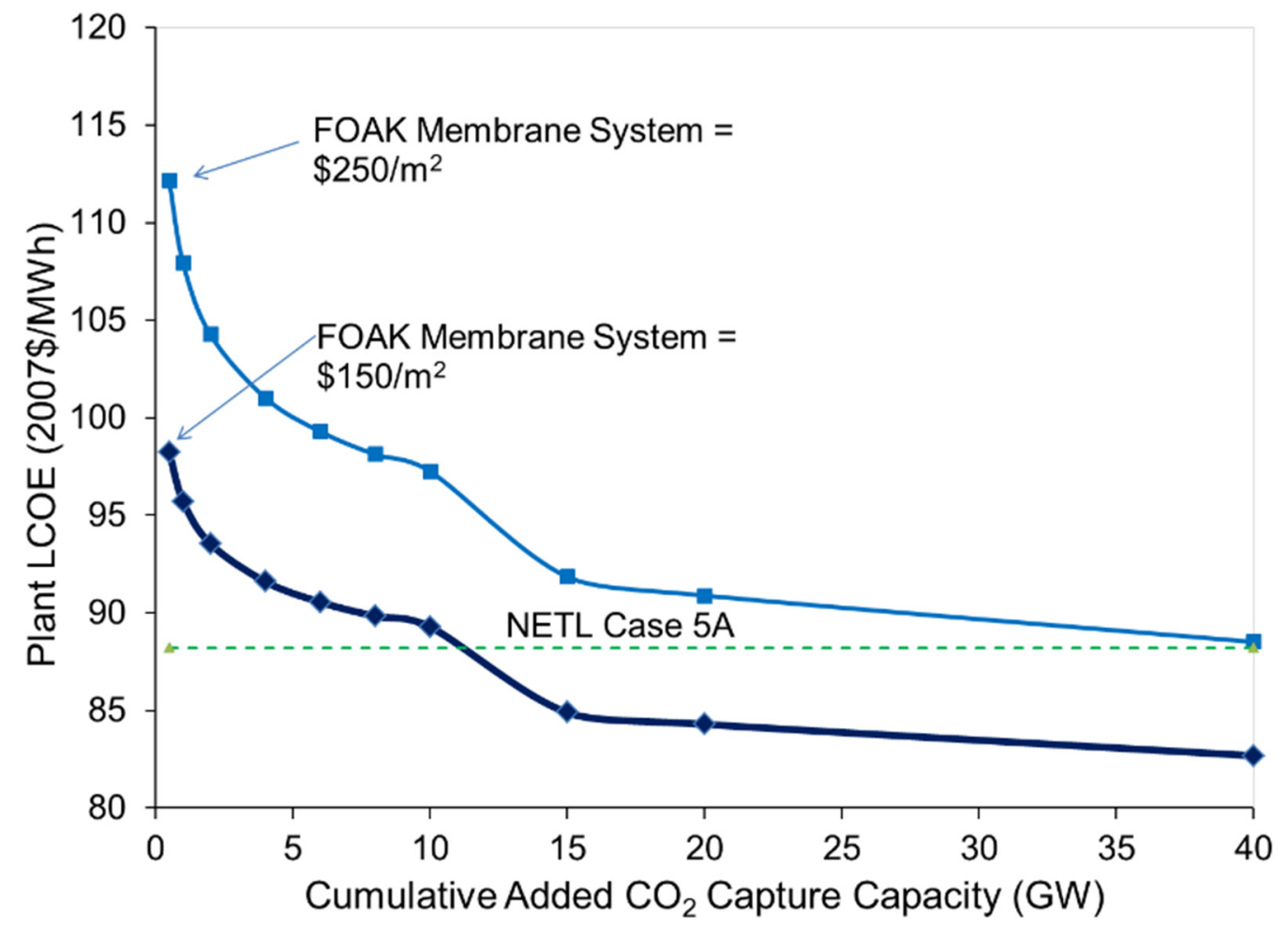

Figure 1-16. Levelised cost of electricity (LCOE) for the two values of FOAK membrane module cost. Financing change from high-risk to low-risk after $10 \mathrm{GW}$ of capture system experience [3]. 
The decline in LCOE is seen to be more rapid after $10 \mathrm{GW}$ of capture capacity experience due to the assumed reduction in risk and financing charges at that point. For the lower FOAK cost estimate, the advanced plant would require about $12 \mathrm{GW}$ of experience (about 24 installations) to reach the NOAK LCOE cost goal (NETL Case 5A). For the higher FOAK cost, substantially more experience (over $40 \mathrm{GW}$ of capacity) would be needed to reach the Case 5A target. NETL Case 5A also assumed a high-risk cost of capital (inconsistent with the premise of a mature NOAK plant). The more appropriate low-risk (commercial) cost of capital would lower the LCOE for Case 5A and thus further increase the cumulative capacity needed for the advanced plant design to reach the NOAK cost goal.

The addition of uncertainty in the learning rate assumptions for this example would further broaden each line in the above figures into a band similar to the one shown in Figure C-2 (Appendix C). In turn, this would further broaden the estimated uncertainty in the projected NOAK cost of the advanced technology. This is illustrated in Figure 1-17, which compares the NOAK capital cost goal in the NETL study to the FOAK cost and the projected "actual" NOAK cost from the hybrid cost analysis. In this illustration, the nominal learning rate of $11 \%$ for the membrane-based capture system is varied from $7 \%$ to $15 \%$, and the $\mathrm{N}^{\text {th }}$-of-a-kind plant is assumed to be reached after five doublings of the initial capture system capacity (in this case, about $18 \mathrm{GW}$ ). Note that because the $\mathrm{CO}_{2}$ capture system accounts for only a fraction of the total plant cost, with little or no reduction in the rest of the plant cost, the percentage reductions in overall cost seen in this example are much smaller than the reduction in capture system cost.

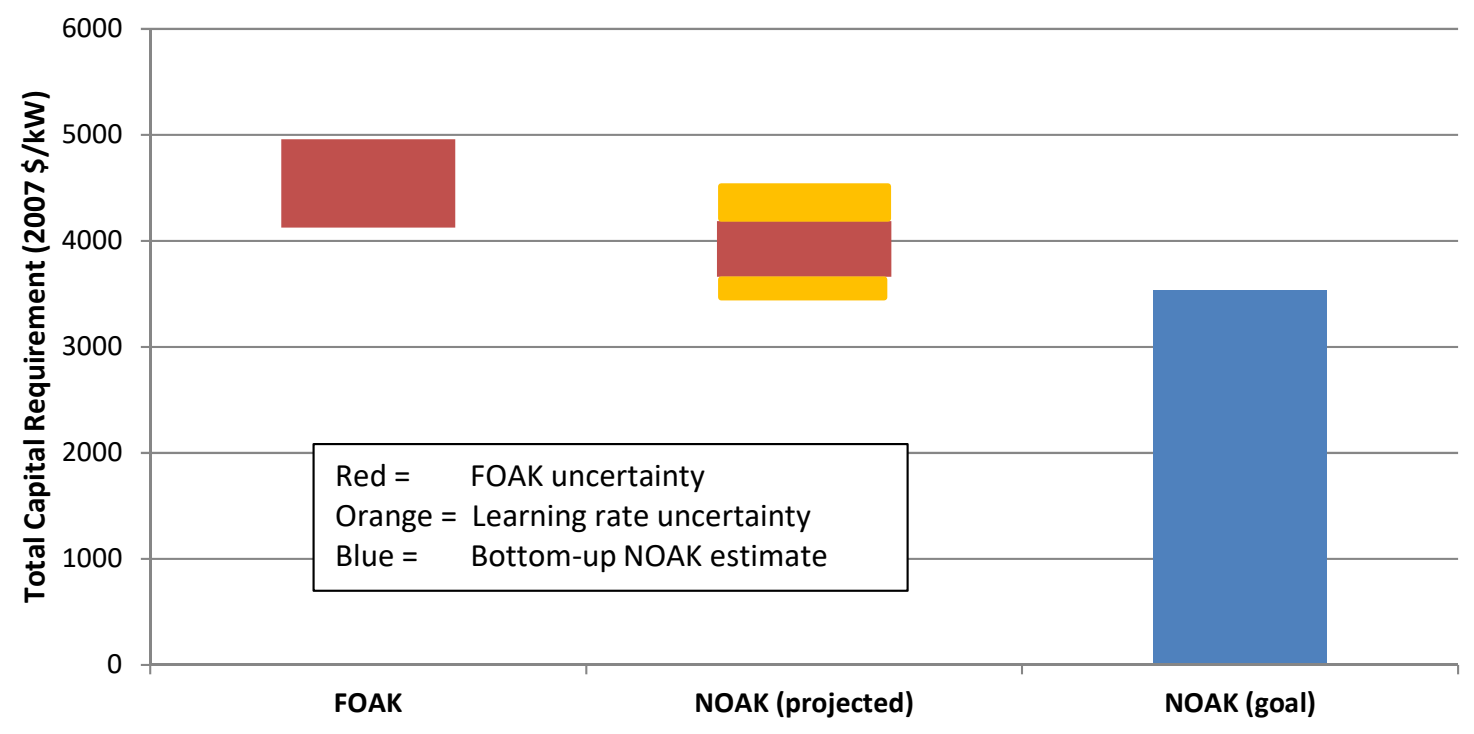

Figure 1-17. Comparison of the total power plant capital requirement for the FOAK plant, projected NOAK plant, and NOAK cost goal including uncertainty in FOAK cost and learning rate for the advanced membrane technology.

Figure 1-17 shows that under the assumptions of this analysis the uncertainties in FOAK cost and capture system learning rate both contribute significantly to the overall uncertainty in projected NOAK cost. Overall, these results indicate that the actual NOAK cost is likely to be significantly higher than the NOAK goal of the bottom-up (What If) cost estimate.

As noted earlier, there is considerable scope to further refine and expand the uncertainty analysis to incorporate additional variables of both the hybrid cost model as well as the engineering-economic model used to generate the NOAK goal. As always, the value of further refinements depends strongly on the purpose of the cost estimate and the decisions or judgments it will be used to inform. In this case, additional efforts to refine the estimated FOAK cost of the advanced membrane module (e.g., via vendor quotes) would be especially helpful given the importance of that estimate for NOAK cost projections. 


\subsection{Discussion and conclusion}

This chapter has presented a framework for estimating the future " $N$ th -of-a-kind" (NOAK) cost of advanced technologies that are currently at early pre-commercial stages of development. That framework distinguishes between two types of question that commonly motivate such a cost analysis: "What If" questions about the hypothetical cost of a technology that meets specified R\&D goals; and "What Will" questions regarding the actual expected cost of an advanced technology once it is mature.

The latter type of question is of particular interest because of the shortcomings in current methods for estimating the expected NOAK cost that were identified in this chapter, as well as the ambiguity in many cost studies as to the meaning of reported NOAK costs. Indeed, the first conclusion drawn from this study is that there is a need for greater clarity as to the question a reported NOAK cost result is intended to answer. Going forward, we recommend that authors of cost studies use the term "NOAK goal" or "aspirational NOAK" when conducting "What If" studies, and the term "expected NOAK" or "projected NOAK" when reporting "What Will" estimates of the future cost of an advanced (precommercial) technology. While some authors may prefer to use other adjectives (including "what if" and "what will"), the term NOAK should not be used in isolation without a clear descriptor.

Regarding "What Will" cost estimates for advanced technologies, the hybrid costing method described in Section 1.2.3, which combines a "bottom-up" engineering-economic analysis with a "top-down" model of technological learning, offers an approach to address the identified deficiencies in current methods. The hybrid cost analysis focuses new attention on the current (first-of-a-kind) commercial cost of an advanced technology. Methods and assumptions for estimating FOAK plant costs vary significantly from those for known commercial technologies, as discussed in Section 1.4 of this report. A bottom-up FOAK cost estimate is recommended as the starting point for projecting future cost reductions of advanced technologies based on historical experience with similar or related technologies. This is embodied in the application of technology experience curves and historical learning rates that underscore the importance of cumulative experience in plant deployments and operation (together with other factors) in achieving future cost reductions. Methods for implementing these cost models were elaborated in Section 1.5 .

As with traditional cost analyses, a variety of tools and methods are available to also characterize uncertainties in cost results and the influence of key decision variables. Three methods - sensitivity analysis, probabilistic analysis, and expert judgments - were discussed and illustrated in this chapter. An uncertainty analysis is especially important for FOAK and projected NOAK costs for advanced technologies because of their intrinsically greater uncertainty compared to well-established commercial technologies. Uncertainty analysis also is important in calculating aspirational NOAK cost goals since these also depend heavily on assumptions and judgments that may vary considerably.

This chapter represents an initial effort to identify needed improvements in current costing methods and to provide guidance for future cost studies, especially for advanced (pre-commercial) technologies. Continuing efforts also are needed to further develop the models, databases, and guidelines that support applications of the hybrid costing method. This includes methodological improvements and quantitative guidelines to improve the development of FOAK cost estimates and reduce the uncertainties in projected NOAK costs for advanced technologies. Despite current limitations, however, the hybrid modelling approach can enhance prevailing assessments of advanced technologies by providing new insights regarding the time frame and requirements for their cost-competitiveness with existing or other advanced technology concepts. Further applications of the hybrid method can thus begin to offer a more complete and realistic approach to assessing the economic potential of advanced energy, industrial and environmental systems, and priorities for R\&D management. 


\section{Chapter 2: Towards improved cost evaluation of carbon capture and storage from industry ${ }^{7}$}

\subsection{Introduction}

\subsubsection{Carbon capture and storage from industry}

The industry sector accounted for over a quarter $\left(9 \mathrm{GtCO}_{2}\right)$ of direct global $\mathrm{CO}_{2}$ emissions in $2019[69]^{8}$. If indirect emissions (i.e. emissions arising from power and heat demand) are included, this sector is responsible for nearly $45 \%\left(16 \mathrm{GtCO}_{2}\right)$ of global $\mathrm{CO}_{2}$ emissions. Despite the historic decline in $\mathrm{CO}_{2}$ emissions in early 2020, caused by the Covid-19 crisis, direct industrial $\mathrm{CO}_{2}$ emissions are expected to rebound as economic conditions improve and continue to grow to around $10 \mathrm{GtCO}_{2}$ in 2060 [70]. Reducing industrial $\mathrm{CO}_{2}$ emissions presents several challenges. One-third of industry energy demand is for high-temperature heat, for which there are few mature and affordable alternatives to the direct use of fossil fuels [71]. Process emissions, accounting for one-quarter (almost $2 \mathrm{GtCO}_{2}$ ) of industrial emissions, result from chemical reactions and therefore cannot be avoided by switching to alternative energy sources [71]. Last, industrial facilities are long-lived assets, leading to potential "lock-in" of $\mathrm{CO}_{2}$ emissions for decades to come. Carbon Capture and Storage (CCS) retrofit is the only technology able to address these challenges and achieve deep emission reductions across the industry sector.

The three highest-emitting industry subsectors in 2019 were iron and steel $\left(2.6 \mathrm{GtCO}_{2}\right)$, cement $(2.4$ $\left.\mathrm{GtCO}_{2}\right)$, and chemicals $\left(1.4 \mathrm{GtCO}_{2}\right)$, together responsible for $70 \%$ of industry's direct $\mathrm{CO}_{2}$ emissions [69]. The complementary share of industrial emissions originates from multiple industrial activities, such as pulp and paper, aluminium, textile, food, and beverages, etc. Industry and fuel transformation (hereafter jointly referred to as "industry") represents a wide variety of processes and $\mathrm{CO}_{2}$ point sources. Among these emitters are high-purity $\mathrm{CO}_{2}$ sources (e.g. natural gas processing, bioethanol production, and hydrogen production), which provide low-cost opportunities for CCS. Furthermore, although some subsectors currently represent a "small" share of global emissions, they may grow rapidly over the coming decades. For example, increased hydrogen production is expected to be a key strategy to decarbonise heat and transport, as well as industrial emissions in certain sectors [71, 72]. Similarly, the waste-to-energy sector is on the rise as both, a waste management option and a heat and power production means [73].

Consequently, the momentum for CCS from industrial sources has accelerated around the world over the past decade [74]. This is especially the case in Europe due to the ambitious mitigation targets of the European Commission to reach carbon neutrality by 2050 [75]. Today, globally 20 large-scale CCS applications at industrial facilities ${ }^{9}$ have entered in operation, while some 24 future large-scale applications are currently at different stages of development [76]. Among these, the Norway full chain project, Longship, is worth mentioning as it will include the first large-scale CCS project in the cement industry and potentially in a waste-to-energy facility ${ }^{10}$. It is worth noting that several of the projects have

\footnotetext{
7 This chapter was authored by Simon Roussanaly, Niels Berghout, Tim Fout, Monica Garcia, Stefania Gardarsdottir, Shareq Mohd Nazir, Andrea Ramirez, and Edward S. Rubin. It corresponds to a post-print version of the paper, "Towards improved cost evaluation of Carbon Capture and Storage from industry." International Journal of Greenhouse Gas Control 106, 103263. 2021" published under a Creative Commons license CC BY and is available in open access at https://doi.org/10.1016/j.ijggc.2021.103263. The corresponding author for this chapter is Simon Roussanaly (e-mail address: simon.roussanaly@sintef.no).

${ }^{8}$ Note that the $9 \mathrm{GtCO}_{2}$ emission number reported by IEA [69] for the industry sector does not include activities related to fuel transformation such as, ethylene oxide, ammonia, and hydrogen production. In the present study, CCS for non-power applications from both industry and fuel transformation is discussed.

9 An application corresponds to the implementation of CCS from a given industrial plant, which means that a CCS project considering a cluster of industries is considered here as multiple applications.

${ }^{10}$ Status in December 2020
} 
been driven by demand for $\mathrm{CO}_{2}$ for EOR operations. The capture and use of $\mathrm{CO}_{2}$ for other purposes than long-term storage (e.g., as an input to the production of fuels, chemicals, and building materials) may also grow over the coming years [77].

\subsubsection{Towards improved cost estimates for CCS from industry}

To support CCS deployment, extensive studies assessing the techno-economic feasibility of CCS from industrial sources have been published, for example, on: iron and steel [78], cement [79, 80], refineries [81], pulp and paper [82], chemical production [83], oil and gas production and natural gas processing $[84,85]$, and hydrogen production [86]. Appendix $\mathrm{F}$ and the supplementary information provide an overview of selected publicly available, transparent, and detailed techno-economic studies for different industrial sectors including key characteristics, assumptions, and results. Although similar capture technologies can be considered in the case for power and industrial applications, their implementation can differ considerably by sector and industrial facility. This is due to differences in, among others, size and properties of the industrial process and gas streams (e.g. $\mathrm{CO}_{2}$ partial pressure), plant layout (e.g. number of point sources and space availability), and energy supply options for the capture process, including the availability of low-value waste heat. Table 2-1 presents typical key plant characteristics for a wide range of industrial processes together with their status on number of existing and planned large-scale CCS deployment.

Different assumptions about these factors are partly responsible for the wide differences in cost estimates for CCS reported in literature, even within a given industrial sector [87, 88]. However, a significant part of the wide ranges in costs reported might arise from other factors, including differences in methodological framework [89], input data quality, cost metric definition, assumptions regarding capture technology maturity, retrofit vs. new-built facilities, plant location, energy prices, waste heat availability, and the inclusion (or exclusion) of $\mathrm{CO}_{2}$ transport and storage. For example, different heat and power supply strategies may be selected resulting in very different $\mathrm{CO}_{2}$ avoided cost $[80,90]$. Furthermore, while CCS retrofit of existing facilities is considered to be an important mitigation measure to decarbonise longlived assets [81], many studies only consider CCS for new-built facilities or underestimate the cost impacts of retrofitting.

A better understanding of the costs of CCS from industry is therefore needed to better inform decisionmakers on the economic potential of CCS and guide research activities to improve the performance of promising options across industrial sub-sectors. Building on a previous CCS costing guideline papers [6], the present work aims to contribute to the development of improved guidelines for cost evaluation of CCS from industrial applications. In particular, the present guideline aims to support the establishment of improved cost evaluation of CCS from industrial applications through three key areas. The first area focuses on cost metrics and challenges that might arise in the case of CCS from industry. The second area focuses on three key underlying cost items for the evaluation of CCS from industrial plants: cost and $\mathrm{CO}_{2-}$ footprint of heat and power consumption; costs associated with implementation of CCS on a retrofit basis, and cost associated with $\mathrm{CO}_{2}$ transport and storage. Finally, the last area focuses on transferability of data, experience, and maturity of CCS from power generation to CCS at industrial sources. 
Table 2-1. Overview of characteristics of key industry subsectors

\begin{tabular}{|c|c|c|c|c|c|}
\hline \multirow[t]{2}{*}{ Industrial subsector } & \multirow{2}{*}{$\begin{array}{l}\text { Indicative contributions to global } \\
\mathrm{CO}_{2} \text { emissions }[\%][69]^{\mathrm{a}}\end{array}$} & \multirow{2}{*}{$\begin{array}{l}\text { Indicative range of } \mathrm{CO}_{2} \text { emissions } \\
\text { from a plant }\left[\mathrm{MtCO}_{2} / \mathrm{y}\right]\end{array}$} & \multirow{2}{*}{$\begin{array}{l}\text { Indicative range of } \mathrm{CO}_{2} \\
\text { concentrations [\% } \% \mathrm{vol}]\end{array}$} & \multicolumn{2}{|c|}{$\begin{array}{c}\text { Large-scale CCS applications at industrial } \\
\text { facilities }^{b}[-][76]\end{array}$} \\
\hline & & & & Existing & Planned $^{\mathrm{c}}$ \\
\hline Cement & $7(2019)$ & $0.7-1[79,91]$ & $14-33[92]$ & - & $2^{\mathrm{d}}$ \\
\hline Iron and Steel & 7 (2019) & $2-14[78]$ & $4-27[78]$ & 1 & - \\
\hline Oil refining ${ }^{\mathrm{e}}$ & $2(2017)$ & $0.7-2.4[93]$ & $8-20[93]$ & 1 & 4 \\
\hline Chemicals & $4(2019)$ & Various $^{\mathrm{f}}$ & Various ${ }^{\mathrm{f}}$ & 4 & 11 \\
\hline Natural gas processing & $2.5[94]$ & $0.5-9[95]$ & $0-70 \% \mathrm{~g}^{\mathrm{g}}$ & 11 & 2 \\
\hline Hydrogen & $2(2018)$ & $0.15-1.3[86,93,96]$ & $15-60 \% \mathrm{o}^{\mathrm{h}}[86]$ & 3 & 5 \\
\hline Offshore oil and gas operations ${ }^{\mathrm{i}}$ & $1.5[97]$ & $0.3-0.6[98]$ & $3-4[85]$ & - & - \\
\hline
\end{tabular}

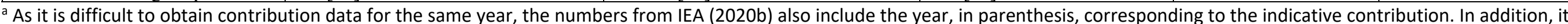

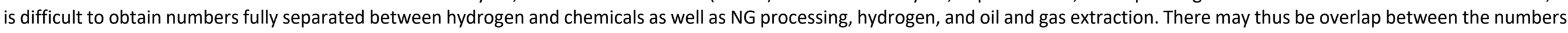
here presented for these industry subsectors.

${ }^{\mathrm{b}}$ When CCS from a cluster of industries exist or is planned, CCS from each of these industries is here reported individually.

c Various level of development are here accounted for (early development, advanced development, completed)

${ }^{\mathrm{d}}$ Note that one of these cement plants correspond to the Norwegian full chain CCS project which may also include $\mathrm{CO}_{2}$ capture from a waste-to-energy plant.

e Hydrogen production units are excluded.

${ }^{f}$ Due to the multitude of industries under this umbrella, it is difficult to provide a meaningful range.

g Considering gas field that reached the production stage.

${ }^{\mathrm{h}}$ Depending on hydrogen production technology (steam-methane reforming or autothermal reforming) and considered capture location.

' Emissions related to heat and power production offshore. 


\subsection{Cost metrics}

Performance metrics and benchmarking are key aspects of technology selection, development, deployment, and improvement. They allow to identify apparent performance gaps and explanatory factors for these as well as best practices that lead to superior performance. Most performance metrics for capture systems are directly derived from power systems. Typical examples are investment costs, incremental products costs, cost of $\mathrm{CO}_{2}$ captured, and cost of $\mathrm{CO}_{2}$ avoided. These costs can be reported in absolute (e.g. Euro) or normalized terms (Euro per tonne $\mathrm{CO}_{2}$ avoided or tonne $\mathrm{CO}_{2}$ captured). Absolute costs are difficult to understand and interpret by non-experts. Without specific background knowledge it is difficult to know whether a given investment value is high or low. Normalized values, i.e., indicators that relate total costs (or investment) to output (e.g., tonne $\mathrm{CO}_{2}$ captured, tonne $\mathrm{CO}_{2}$ avoided, tonne of product(s), increase in production cost of industrial product) or input (e.g. per MJ fuel input) are often used as they allow (under similar assumptions) to compare and/or benchmark technologies.

\subsubsection{Common metrics}

The most common metrics to evaluate $\mathrm{CCS}$ are cost of $\mathrm{CO}_{2}$ captured and $\mathrm{CO}_{2}$ avoidance cost (also referred to as cost of $\mathrm{CO}_{2}$ avoided) [6]. The former provides insights into the investments and operating costs associated with the $\mathrm{CO}_{2}$ capture (or the CCS chain) while the latter gives insights into the performance of the capture unit (or the CCS chain) as a carbon mitigation option. Cost of $\mathrm{CO}_{2}$ captured (Euro per tonne of $\mathrm{CO}_{2}$ captured) relates the costs needed for building and operating the capture and compression units (or the whole CCS chain) to the physical amount of $\mathrm{CO}_{2}$ captured and compressed from a given point source. Note that in most cases, $\mathrm{CO}_{2}$ captured costs do not include the costs of transport and storage. Nor does it consider the $\mathrm{CO}_{2}$ emitted from process energy supply. $\mathrm{CO}_{2}$ avoidance costs (euro per tonne of $\mathrm{CO}_{2}$ avoided) is the most common and meaningful metric used when assessing the costs of CCS as an abatement option as it provides insights into the costs of not emitting one tonne of $\mathrm{CO}_{2}$ to the atmosphere while still producing a unit of useful product ${ }^{11}$. Therefore, it can be used to compare different types of CCS systems when assessing the most effective option to reduce $\mathrm{CO}_{2}$ emissions from a given process. Note that the design of a capture unit, a compression unit, or a pipeline is based on the amount of emissions captured not avoided.

$\mathrm{CO}_{2}$ avoidance cost $(\mathrm{CAC})$ is a relative metric and therefore it requires a reference system (see equation 7). For the result to be rigorous, it is important that the industrial facility produces the same amount of key material output for both systems (with CCS and without CCS). Furthermore, CAC takes into account that operating the capture and compression unit requires energy and materials thereby producing additional indirect $\mathrm{CO}_{2}$ emissions. In general, an industrial plant captures more $\mathrm{CO}_{2}$ than it avoids, and therefore the costs per tonne of $\mathrm{CO}_{2}$ captured are lower than the costs per tonne of $\mathrm{CO}_{2}$ avoided.

Three different calculation methods can be used to evaluate the CAC in the case of CCS from industrial sources: the so-called "exhaustive" method, the "net present value method", the "annualization calculation method" (Roussanaly, 2019). The "exhaustive" method is shown in Equation 7 while the equations used for calculating the $\mathrm{CAC}$ in the other two methods are presented in Appendix G.

$$
C A C=\frac{(L C O K M)_{c C S}-(L C O K M)_{r e f}}{\left(t_{C O 2} / U_{K m}\right)_{r e f}-\left(t_{C O 2} / U_{K m}\right)_{c c s}}
$$

Where, LCOKM is the levelised cost of the key material(s) of the industrial plant with CCS or without in, for example, $€$ per unit of key materials(s) [99], $\left(t_{\mathrm{CO}_{2}} / U_{\mathrm{Km}}\right)$ is the mass amount of $\mathrm{CO}_{2}$ emitted per unit of key material(s) with or without CCS.

\footnotetext{
11 This means in practice that the emissions associating with building and operating the $\mathrm{CO}_{2}$ capture facility (CCS chain) are also taken into account.
} 
While the exhaustive method is always valid, it is worth noting that the two other methods do not require the assessment and evaluation of the considered industrial plant hence requiring significantly less effort and data. However, these two approaches also come with limitations and therefore must be used carefully. A summary of assumptions required to ensure the validity of each $\mathrm{CO}_{2}$ avoidance cost calculation methods is presented in Appendix G.

Note that the CAC is often presented together with a breakdown of cost along the CCS chain, withCO $\mathrm{O}_{2}$ conditioning either lumped together with the capture or transport steps. However, it is recommended here that any cost breakdown of CAC present capture, conditioning, transport, and storage as four individual items.

In power plants, CAC includes the impact of the capture unit on the efficiency of the power plant (as a consequence of using part of the steam and/or generated in the plant to cover the energy needs of the capture unit). In most industrial settings, however, $\mathrm{CAC}$ needs to include the costs and $\mathrm{CO}_{2}$ emissions from additional units e.g., a boiler or a combined heat and power (CHP) system needed to cover the energy requirements of the capture and compression units, or emissions associated to the use of electricity from the electricity grid. Note that not only additional units but also changes in existing units as a consequence of $\mathrm{CO}_{2}$ capture need to be taken into account. For instance, if the capacity of an existing boiler is increased so it can supply steam to the capture unit, the additional fuel (and related emissions) needs to be allocated to the capture unit and accounted for in the CAC calculation. If, however, waste energy is available at location and no extra units or extra capacities in existing units are required then the costs of $\mathrm{CO}_{2}$ avoided could be equal to the cost of $\mathrm{CO}_{2}$ captured (including the cost incurred to utilise the waste heat for $\mathrm{CO}_{2}$ capture). This only holds if the "waste energy" is really so in practice. Many studies use average amounts of waste energy taken from e.g., literature, and therefore tend to overestimate the amount of waste energy available on-site ${ }^{12}$.

CAC is generally used to estimate the minimum $\mathrm{CO}_{2}$ emission penalty (tax or quota price) that would be required for making a point source without $\mathrm{CO}_{2}$ capture as expensive as a point source with $\mathrm{CO}_{2}$ capture (or to estimate the subsidy required to make a point source with $\mathrm{CO}_{2}$ capture as expensive as a similar point source without it). An important aspect to highlight here is the importance of system boundaries in the calculation of CAC. Strictly speaking, because we are interested in the costs of not emitting one tonne of $\mathrm{CO}_{2}$ into the atmosphere all emissions should be included, that is direct emissions (i.e. emissions generated in the plant or so-called gate-to-gate emission) and indirect emissions (i.e. emissions taking place outside the industrial plant, for instance during the extraction and transport of fuels, during the production of electricity, the transport and storage of $\mathrm{CO}_{2}$, or the end life of the product). The wider the system boundaries the lower the avoided emissions and the higher the CAC.

Another indicator that is generally used is incremental production costs per unit of product. It relates the costs of capture to the net output of a facility. In a power plant, the impact of capture in the production of electricity is generally assessed through the energy efficiency penalty induced by the capture unit. In an industrial plant, this is less obvious for two reasons. First, it depends on the origin of the energy used to supply the capture requirements e.g., an extra boiler, waste energy, or existing steam. In the latter case (use of existing steam) the capture system may impact the costs and or performance of the unit where the steam was originally used. This impact needs to be assessed and included in the calculation. Second, a key distinction between the power and industrial sector is that most industrial processes do not produce one product and require allocating the incremental costs to the different products. This is discussed in detail in the next section.

\footnotetext{
${ }^{12}$ At a given site, however, the energy is for instance not wasted but is actually used to for instance pre-heat a flow, raw material pre-treatment, or for district heat. In such cases, the impact of taking this energy taken away (to be used in the capture unit) should be included in the calculations (for instance by including emissions that will be incurred when replacing the heat source).
} 


\subsubsection{The challenge of multi-products in industrial sectors}

A key distinction between the power and industrial sector is that many industrial processes produce multiple outputs in a unit. In an atmospheric distillation unit at a refinery for instance, LPG, Naphtha and Diesel/Kerosene are produced. If $\mathrm{CO}_{2}$ capture would be applied to such a unit, the metric to report the cost of product is not straightforward, as it can be referred to one or more products, including intermediate ones.

This problem is not unique to carbon capture and has been discussed when estimating costs of multiproduct industries. A typical approach is to distinguish between joint costs (i.e. costs of a production process that yields a number of products where a physical relation exists between the products that prevents one from being obtained independently from the others) and separable costs (i.e. cost incurred in processes that produces a single product) [100]. To separate joint costs from separable costs, a split-off point is required as indicated in Figure 2-1. Separable costs are therefore all the costs incurred beyond the split-off point (for instance, cost required for purifying a given product). The costs related to the production of a product (for instance product A) are therefore composed of part of the joint costs plus the separable costs of the product. In the simplest joint processes, the joint products are sold at the split-off point (no further processing is required such as for by-product BPc in the figure) and the separable costs are zero.

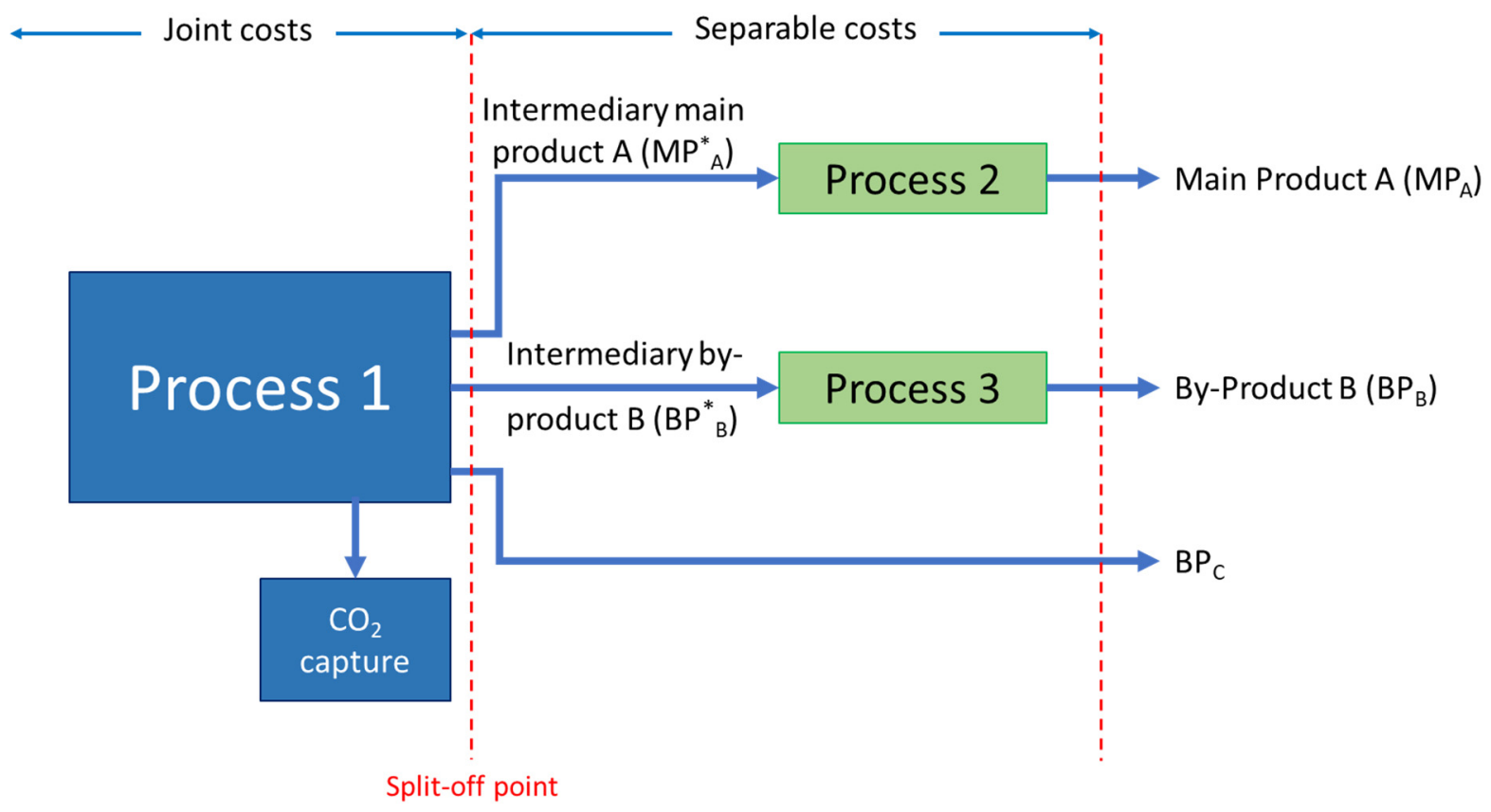

Figure 2-1. Illustration of joint vs separable costs.

There is, however, no standardized methodology currently available to determine the contribution of the different production factors (energy, water, labour) used in the production of each of the joint products at the split-off point. Because in the case of joint costs, one product cannot be produced without the other, it is not physically possible to measure the costs of production factors used in the manufacture of each of the joint products. Companies use different methods to allocate the costs to the joint products. In general, all production costs need to be allocated to all products and to do so, companies distinguish between main product(s) and by-products. This distinction is generally made based on the portion of their sales in the total sales of the company. A main product is a product (or products) with significantly higher total sales values compared to the total sales values of other products while by-products are products of a joint process that have low or no total sale value compared with the total sale value of the main product(s). The classification of products (main product or by-product) changes over time and among companies. 
The costs allocated to the main products are generally estimated by either:

- Allocating the costs according to the amount of product produced defined by physical measures such as the share of mass content (the ratio between the total annual production of each product and the total annual refined oil products). This method requires that all the products be measured with the same underlying physical measure (weight, volume, quantity, etc) and is recommended when the different products are homogeneous and their sale prices are relatively close to each other.

- Allocating the costs according to market-based information such as the sale prices of the products. It is based on the assumption that the market price is a proxy for the production costs. In this method, joint costs are, for instance, allocated to the main products proportionally to the sum of the final sales value of the output. Note that if one of the main products cannot be sold as it is (for instance an intermediate product for which there is not known market price and therefore further processing is needed before a main product can be sold in the market), the price incurred to finish the product (separable costs) is subtracted from the sales value of the product. This is called net realizable value and is a hypothetical market value at intermediate stage of production which assumes that all the profit margin is attributable to the joint process and not to the separable costs. Assuming that the capture unit was applied before the split-off point (i.e. part of the joint cost as shown in Figure 2-1), it is worth noting that the larger the separable costs are, the lower is the contribution of the $\mathrm{CO}_{2}$ capture unit to the total product costs and vice versa.

The costs of by-products can be estimated by:

$\circ$ Assigning them no value (costs are therefore only allocated among main products). This method is also known as the Miscellaneous income method. Note that this approach used to be the default approach used to the $\mathrm{CO}_{2}$ from the capture unit in which no costs were allocated to the $\mathrm{CO}_{2}$ and all costs were allocated to the main product (e.g., steel or electricity). However, in cases where new options emerge that provide economic value to the $\mathrm{CO}_{2}\left(\mathrm{CO}_{2}\right.$ utilization), an appropriate (case-specific) non-zero value should be assigned. Consequently, even using the same methodology, the estimated costs might vary as these are a function of the final use of the $\mathrm{CO}_{2}$ and the market for that use. Furthermore, and depending on the $\mathrm{CO}_{2}$ sales price, there may be cases where the $\mathrm{CO}_{2}$ may be considered a main product instead of a by-product. In such cases, one must be careful to distinguish between uses of $\mathrm{CO}_{2}$ that result in permanent (long-term) removal from the atmosphere ${ }^{13}$ - as required for GHG mitigation - versus utilization that soon results in the release of $\mathrm{CO}_{2}$ to the atmosphere and thus does not contribute to GHG mitigation.

- Assigning them a net realizable value. In this case, the value of selling the product is large enough to have a significant effect in the profits of the company but not large enough to be comparable to the profit of the main product. Note that by allocating costs to the by-product, a reduction in the production costs of the main product(s) will be shown (as the total costs of producing the main products and the by-products is constant).

- Assigning them a net realizable value minus a given profit value

$\circ$ Assigning them the expenditure allocated to the acquisition price or the replacement value on the current market (for instance when a by-product is used within the plant to avoid purchasing materials or utilities, for example, the combustion of a waste to provide heat which avoids purchasing natural gas)

Currently, there are no standardized guidelines of best available practices for selecting allocation methods and, in practice, each company has its own internal approach. To be able to compare capture costs that have been allocated to a given product (including $\mathrm{CO}_{2}$ that will be used by a third party for $\mathrm{CCU}$ ) explicit

\footnotetext{
${ }^{13}$ It is worth noting that, in such cases, $\mathrm{CO}_{2}$ utilization could still contribute to reduction of fossil fuel use elsewhere in the overall chemicals manufacturing industrial system. Under specific conditions, this reduction may be considered as contributing to GHG emissions mitigation.
} 
documentation needs to be provided in the number and characteristics (type, amount, concentration, etc) of products and by-products as well as a detailed description of the approach used for allocation. When possible, it is recommended to examine the costs using more than one allocation method as this will provide insights into the impact of the method in the results.

Finally, it is worth noting that similar questions and approaches are considered in life cycle analysis [101]. It is thus important to ensure consistency between techno-economic and life cycle analyses on this point when performing considering cost and emission allocations in multi-products industrial plants [102].

\subsection{Considerations for improved assessment of key cost contributors}

This section discusses three key contributors to the cost of CCS from industry, which are often not studied in adequate detail are examined and exemplified: 1) energy aspects 2) retrofitting costs 3) $\mathrm{CO}_{2}$ transport and storage costs.

\subsubsection{Energy aspects}

$\mathrm{CO}_{2}$ capture from industrial processes is typically energy-intensive and thus a large part of the $\mathrm{CO}_{2}$ capture cost is potentially related to the use of energy. For example, the steam consumption for MEA solvent regeneration in a cement plant typically contributes to nearly $50 \%$ of the $\mathrm{CO}_{2}$ capture cost ${ }^{14}$ [80]. Heat (mainly in the form of steam) and electricity are the two main forms of energy needed by $\mathrm{CO}_{2}$ capture processes. The form of input energy differs per capture technology and facility; for example, oxyfuel and membrane-based systems use electricity while chemical absorption systems require both heat and power. In practice, several key factors determine the cost and $\mathrm{CO}_{2}$ emissions associated with energy consumption: type of energy used (electricity and heat), origin and supply strategy of energy, costs and emissions intensity of the primary energy source, and possibility to export excess energy to third parties. Most of these factors are region- and facility-specific and may not only affect the $\mathrm{CO}_{2}$ avoidance cost but also the comparison of $\mathrm{CO}_{2}$ capture technologies. Therefore, in studies related to $\mathrm{CO}_{2}$ capture in industrial processes, it is recommended to discuss the sensitivity of the $\mathrm{CO}_{2}$ avoidance costs with respect to the choice of facility characteristics, fuel prices, energy supply, and export alternatives. The effect of the choice of energy supply alternatives is discussed below with an example on $\mathrm{CO}_{2}$ capture in a cement plant.

\subsubsection{Energy supply strategies}

The origin and production/supply strategy of steam and electricity have a significant impact on their production costs and associated $\mathrm{CO}_{2}$ emissions, and may thus significantly impact the $\mathrm{CO}_{2}$ avoidance cost. While a given heat and power supply strategy is often implicitly adopted in the evaluation of $\mathrm{CO}_{2}$ capture technologies, it is important to realise that this implicit assumption may impact significantly the $\mathrm{CO}_{2}$ avoidance cost of a capture technology as well as the comparison of capture technologies.

Figure 2-2 illustrates different steam and electricity supply strategies in the case of a cement plant integrated with an MEA-based absorption $\mathrm{CO}_{2}$ capture unit [80], while Table 2-2 presents their associated costs and $\mathrm{CO}_{2}$ emissions intensities. As seen in Table 2-2, extracting steam from a low-pressure turbine or steam originating from waste heat recovery in core industrial processes are cheaper options and have lower $\mathrm{CO}_{2}$ emissions intensity. Thus, integrating excess heat available in the industrial plant or in another facility near the $\mathrm{CO}_{2}$ capture unit is expected to be a cost-effective solution. However, it should be noted that a large amount of good quality waste heat is rarely available in industrial plants and should be expected to be further driven down by energy efficiency improvement efforts. On the other hand, an electric boiler can in general appear as an inefficient way of producing steam for the $\mathrm{CO}_{2}$ capture unit. However, it is important to also realise that the cost and associated emission intensity of a steam strategy depends on the site-specific characteristics, as well as external parameters such as energy prices.

${ }^{14}$ When a natural gas boiler is used to produce steam and the waste heat from the original facility available to invest on the $\mathrm{CO}_{2}$ capture system is limited. 


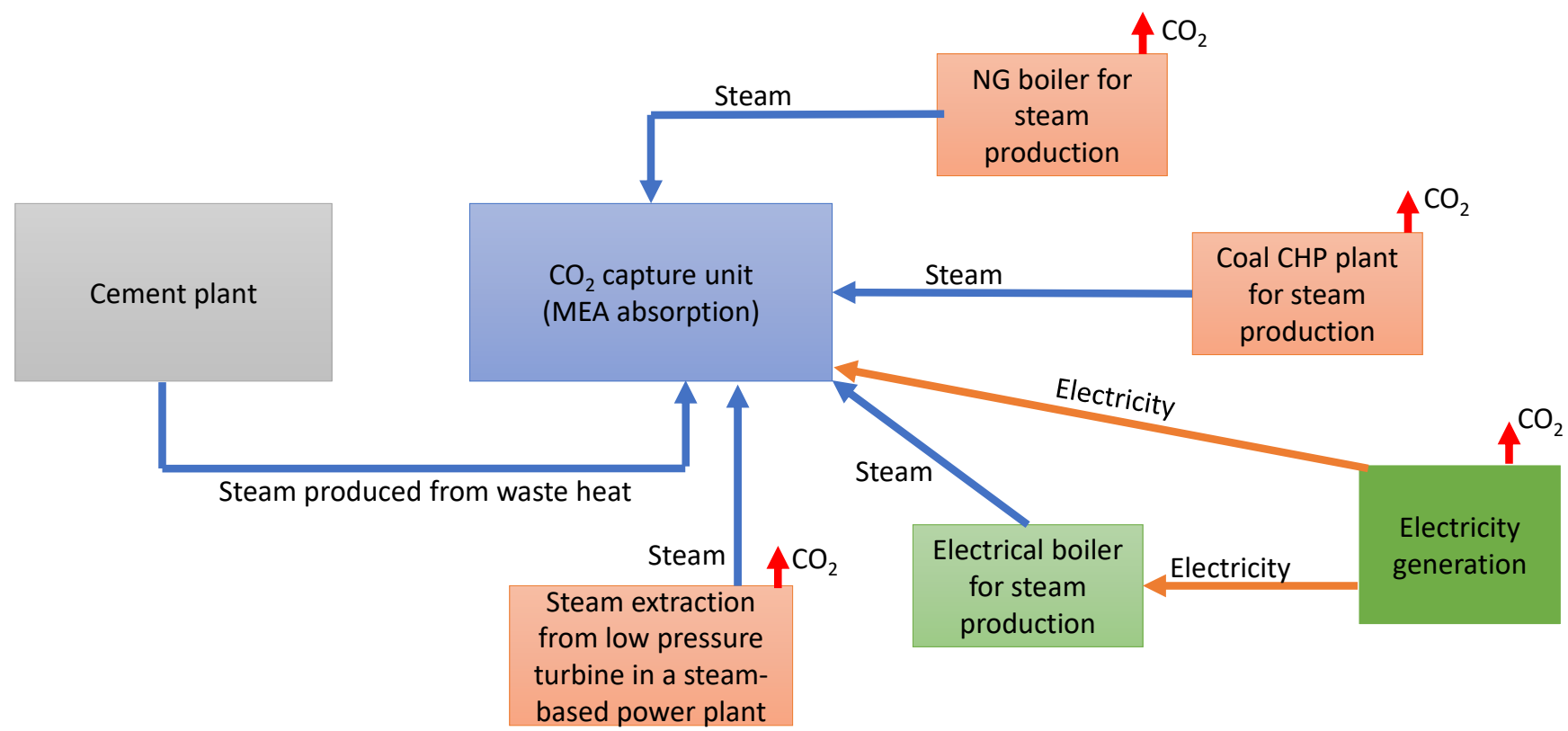

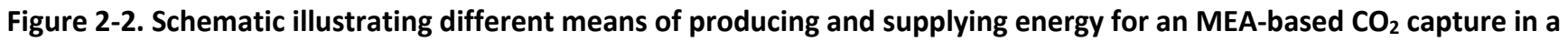
typical cement industry.

Table 2-2: Cost and $\mathrm{CO}_{2}$ emission intensity of different stream supply options

\begin{tabular}{|l|c|c|}
\hline Source & $\begin{array}{c}\text { Emission intensity } \\
{\left[\mathrm{kgCO}_{2} / \mathrm{GJ}\right]}\end{array}$ & $\begin{array}{c}\text { Steam cost } \\
{\left[\epsilon_{2015} / \mathrm{GJ}\right]}\end{array}$ \\
\hline Electric boiler & 87 & 18 \\
\hline Natural gas boiler & 57 & 7.2 \\
\hline Natural gas-CHP plant & 57 & 6.4 \\
\hline Coal CHP plant & 127 & 6.1 \\
\hline Steam extraction from an LP Turbine & 49 & 3.7 \\
\hline Excess heat from industrial core process & 0 & 1.9 \\
\hline
\end{tabular}

Table footnote: These costs are based on the heat supply evaluation performed in the CEMCAP project. [80, 90]. These were established for a generic Netherlands-based application, in the context of $\mathrm{CO}_{2}$ capture from a cement plant, with an NG price of $6 € / G J$, a coal price of $3 € / G J$ and an electricity price of $58 € / \mathrm{MWh}$, and a $\mathrm{CO}_{2}$-intensity associated with electricity consumption of $306 \mathrm{gCO}_{2} / \mathrm{MWh}$. A project duration of 25 years and a real discount rate of $8 \%$ are considered.

\subsubsection{Impact of energy prices}

The cost of the energy supply is directly linked with the price of input energy sources (fuel or electricity). However, the energy source prices can vary significantly based on local market conditions, local environmental policy framework, and their possible future evolutions [103]. It is thus important to also understand the impact of these energy source prices on the steam production cost.

Figure 2-3(a, b, c) shows an example of a sensitivity analysis of the steam cost as a function of the price of coal, natural gas, and electricity respectively. As can be seen from these, the energy prices can significantly impact the selection of an optimal steam production strategy. Overall, steam extracted prior to an LP turbine or generated based on available waste heat remain the cheapest options in most situations. However, if electricity prices are high, gas- or coal-CHP can be very attractive options as these would also result in the production of high-value electricity.

Finally, it is important to realise that some of these energy prices are linked. For example, higher global coal and gas prices can be expected to lead to higher global electricity prices. As a result, a heat and power production unit used for $\mathrm{CO}_{2}$ capture and also selling excess electricity might be less impacted by an increase in fuel prices due to high electricity revenues. Similarly, carbon prices/taxes increase overtime to penalise $\mathrm{CO}_{2}$ emissions can also significantly impact the performance and selection of heat and power 
supply strategies. A possible way to deal with these uncertainties is to make scenarios about future plausible combinations of energy and carbon prices to clearly understand the variety of possible outcomes.

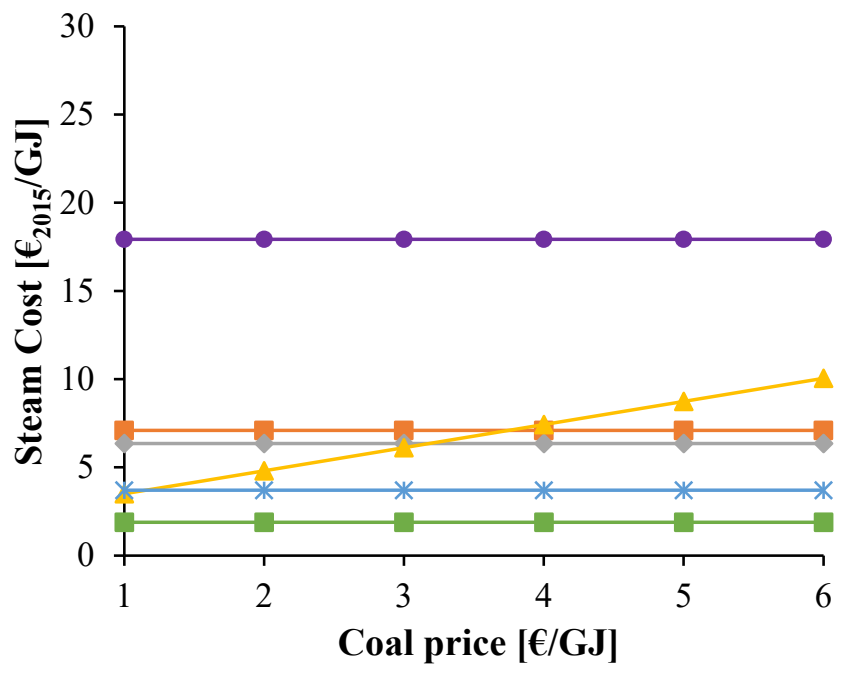

(a) Impact of coal price

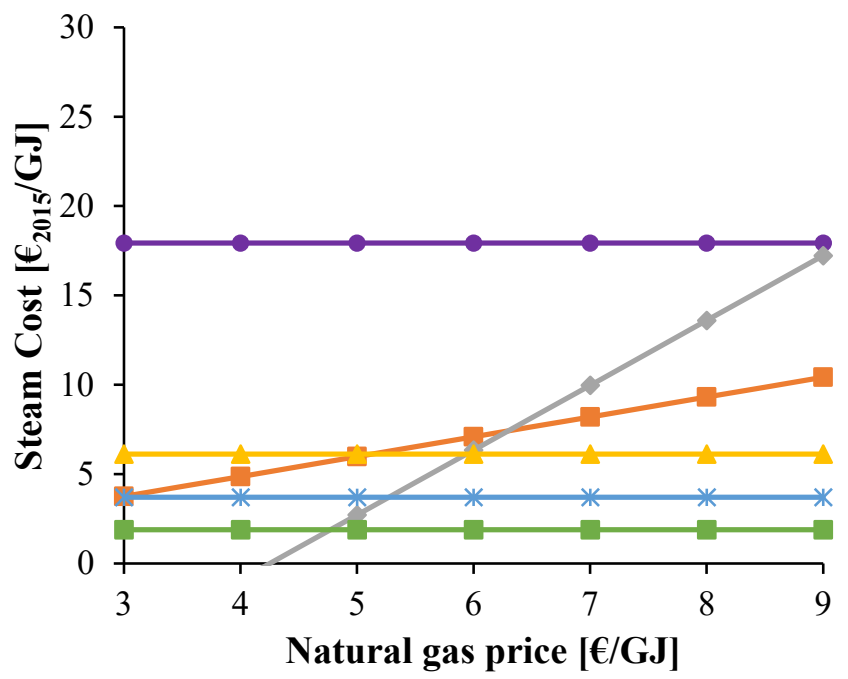

(b) Impact of natural gas price

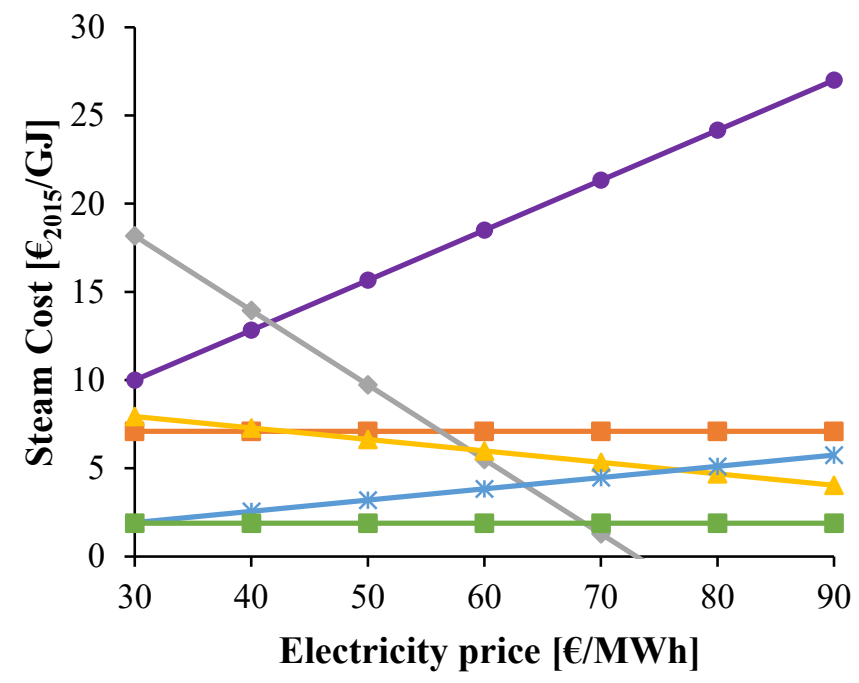

(c) Impact of electricity price

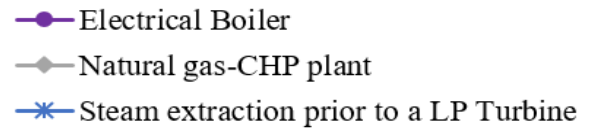

$$
\begin{aligned}
& - \text { - Natural gas boiler } \\
& -- \text { Coal-CHP plant } \\
& - \text { - Waste heat }
\end{aligned}
$$

Figure 2-3. Impact of energy prices on the steam cost for different steam supply strategies: a) coal price b) natural gas price c) electricity price

Figure footnote: Results included in these figures were calculated on the same basis as Table 2-2, while the energy (coal, natural gas, electricity) prices are here varied to understand their impact on the steam cost.

\subsubsection{Credits for import and export of energy}

In some cases, energy is imported or exported from the industrial site due to the implementation of $\mathrm{CO}_{2}$ capture, resulting in a change in energy production and consumption, and related $\mathrm{CO}_{2}$ emissions produced elsewhere in the energy system (indirect emissions). Similarly, the economics of the capture case may be impacted due to the purchase or sale of energy from or to third parties.

One way to account for these effects is to assign credits or penalties to the costs and $\mathrm{CO}_{2}$ emission reductions in the $\mathrm{CO}_{2}$ capture case. Cost and environmental penalties or benefits from the energy import or export will be site- and region-specific, and will also depend on the reference case without $\mathrm{CO}_{2}$ capture [88]. For example, the potential electricity exported to the grid will displace electricity generation 
elsewhere in the broader energy system; this can for example be electricity generated in a coal-fired power plant or renewable electricity, each with a different carbon-intensity. Credits for emission savings elsewhere in the broader energy system will depend on the $\mathrm{CO}_{2}$ emissions factor of the electricity grid, and can therefore vary significantly from one region to another.

The credits can be calculated by multiplying the imported or exported energy with an energy price and $\mathrm{CO}_{2}$ emission factor based on life cycle analysis, which best reflect the reference case. In many cases, this is a fair approach, especially when it concerns the export of excess fuel and steam coming from the core industrial process, which may have changed due to the $\mathrm{CO}_{2}$ capture process. However, this approach is arguably less fair when considering the export of steam or electricity from newly built energy plants. After all, the generation and export of large amounts of electricity and steam could create economic revenues and emissions savings (if it displaces more carbon-intensive energy elsewhere in the broader energy system), and not allocating these credits to the $\mathrm{CO}_{2}$ capture case could distort its techno-economic performance [104]. An alternative accounting method is to exclude revenues from excess electricity and steam generation as well as the costs for the share of the fossil fuel or biomass of the energy plant that corresponds with this excess energy production (which can be determined on an exergy, energy, or economical basis). While there is not an inherently best accounting method, this can have a large impact on the computed avoidance costs and emissions of the capture case. Study practitioners should thus be explicit on their considered accounting method.

\subsubsection{Effect of energy aspects on $\mathrm{CO}_{2}$ avoidance costs and choice of capture technology}

To illustrate the effect of energy supply strategy on the $\mathrm{CO}_{2}$ avoidance costs and comparison of two capture technologies (MEA-based absorption and membrane-assisted liquefaction) in a cement plant [80], an example of 7 scenarios are presented in Figure 2-4 in order to highlight the energy aspects discussed above. In particular, these scenarios combine different steam supply strategies (natural gas boiler, extraction prior to a low-pressure steam turbine, electricity boiler), electricity prices (30 and $80 € / \mathrm{MWh}$ ), and natural gas prices ( 6 to $9 € / \mathrm{GJ}$ ). While none of these scenarios consider $\mathrm{CO}_{2}$ capture from ancillary energy supply unit(s), it is worth noting that this together with switching to bio-based energy are key to reach deep emissions reductions across the industrial process [105]. However, $\mathrm{CO}_{2}$ emitted from these ancillary units should be captured and geologically stored as well to achieve deep levels of decarbonisation, and these costs should be represented in the overall $\mathrm{CO}_{2}$ avoidance cost.

As can be seen in Figure 2-4 the selected steam supply strategy can have a significant impact on the technology comparison. In the heat supply scenarios, MEA-based is the most cost-efficient capture technology in the natural gas boiler and steam extraction prior to a LP turbine scenario (respectively scenarios 1 -also referred as base case- and 2). On the other hand, the membrane-assisted liquefaction is the most cost-efficient capture technology if steam must be supplied through an electric boiler (scenario 3 ). It is also worth noting that the $\mathrm{CO}_{2}$ avoidance cost of the cost-optimal capture technology may or may not be impacted. For example, compared to the natural gas boiler scenario, the steam extraction scenario results in significantly lower $\mathrm{CO}_{2}$ avoidance cost for the optimal capture technologies, while the electric boiler scenario result only in slightly lower costs.

Figure 2-4 also illustrates that energy prices can also have an impact on the $\mathrm{CO}_{2}$ avoidance cost, as well as on the comparison of technologies. Compared to the base case, a higher natural gas price favours the membrane-assisted liquefaction (scenario 4) while a higher electricity price would favour the MEA-based capture (scenario 5). The potential impact of $\mathrm{CO}_{2}$ emissions associated with heat and electricity consumption can also be visualised by comparing the scenarios 3 and 6 (both based on heat supply through an electric boiler). As electricity is assumed to be based on renewable source in this scenario, no $\mathrm{CO}_{2}$ emissions are associated with the consumption of heat and electricity thus resulting in lower $\mathrm{CO}_{2}$ avoidance cost. Finally, scenario 7 combines a reduction in electricity prices and no $\mathrm{CO}_{2}$ emissions associated with power. In this case, steam produced from an electric boiler results in lower $\mathrm{CO}_{2}$ avoidance cost than the base case (scenario 1) as well as nearly on par $\mathrm{CO}_{2}$ avoidance costs between the two $\mathrm{CO}_{2}$ 
capture technologies. Although scenario 7 may seem far-fetched for most locations, it is representative of conditions for $\mathrm{CO}_{2}$ capture in Norwegian industrial plants ${ }^{15}$.

Building on the energy aspects discussed previously, these scenarios further emphasize the importance for TEA practitioners to consider the impact of possible energy supply strategies as well as possible evolution of global energy scenarios.

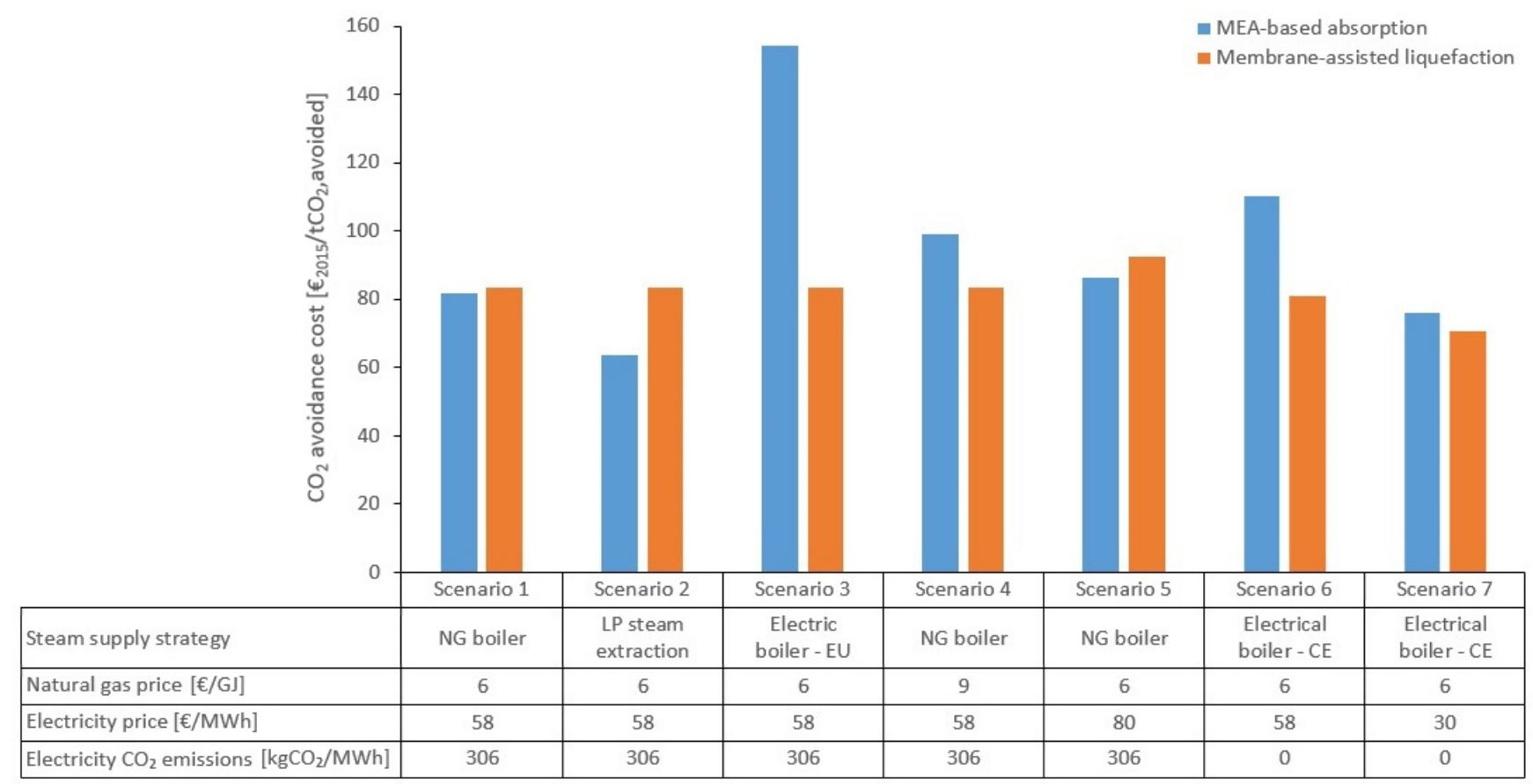

Figure 2-4. Illustration of the impact of different energy scenarios on the $\mathrm{CO}_{2}$ avoidance costs and the comparison of two capture technologies (MEA-based absorption and Membrane-assisted liquefaction)

Figure footnote: The performance of the MEA-based and membrane assisted liquefaction for $\mathrm{CO}_{2}$ capture from a cement plant are extracted from the CEMCAP project [80]. These were established for a generic Netherlands-based application, a project duration of 25 years, and a real discount rate of $8 \%$.

"NG boiler" corresponds to natural gas boiler; "LP steam extraction" corresponds to steam extraction prior to a low-pressure turbine; "Electric boiler - EU" corresponds to electric boiler powered by electricity with the characteristics of the average European Union electricity mix; "Electric boiler - CE" corresponds to electric boiler powered by the clean electricity thus it is assumed to not result in any $\mathrm{CO}_{2}$ emissions.

\subsubsection{Retrofitting costs}

In retrofitting an industrial plant with a $\mathrm{CO}_{2}$ capture process, several plant-specific and technologyspecific characteristics can entail significant costs and considerations for the $\mathrm{CO}_{2}$ capture process that are often overlooked in techno-economic studies of industrial CCS applications. This section aims to highlight some of the most important retrofitting cost aspects and provides several numerical examples for illustrating these.

\subsubsection{Economic impact of plant production stop for $\mathrm{CO}_{2}$ capture retrofit}

Retrofitting of $\mathrm{CO}_{2}$ capture technologies at an industrial plant might involve a temporarily shut down, fully or partially, especially if fundamental modifications to the core process are required. The resulting production losses can have non-negligible economic consequences, depending on the type of process, integration of the $\mathrm{CO}_{2}$ capture system with the original facility, and economies of scale, and will therefore have to be kept to an absolute minimum. For certain industrial applications and $\mathrm{CO}_{2}$ capture technologies, e.g. oxyfuel or pre-combustion technologies in cement or iron and steel applications, a significant downtime might be required to modify the existing industrial plant for deep integration with the $\mathrm{CO}_{2}$

\footnotetext{
${ }^{15}$ Although the $\mathrm{CO}_{2}$ footprint would not be zero if assessed through a full life cycle analysis.
} 
capture plant. For other end-of-pipe technologies that do not require significant integration with the core process, other than re-routing of the flue gas, it could be expected that the retrofit period is aligned as much as possible with a routinely scheduled production stop for maintenance to minimize the economic impact. In some cases, it might be possible to only shut down parts of the core plant for the retrofit and thereby avoiding $100 \%$ production losses, e.g. in a plant with several emission sources where only a single source is retrofitted with $\mathrm{CO}_{2}$ capture or a plant with multiple production lines. This could be the case in a modern steel mill or a multi-product oil refinery where the production process is not necessarily linear. Although not exemplified here, it should be mentioned that the same considerations for plant production stop and economic consequences also apply in retrofitting $\mathrm{CO}_{2}$ capture to power plants. In any case, costs arising from plant production stop should transparently be taken into account in estimating the costs of $\mathrm{CO}_{2}$ capture.

The economic impact of production stops for retrofit is exemplified below for three different industrial plants: a cement plant [80, 106], a pulp mill [82], and an integrated steel mill [78]. Key data for these industrial plants is listed in Table 2-3.

The added cost of $\mathrm{CO}_{2}$ avoided due to a production stop for $\mathrm{CO}_{2}$ capture retrofit for the three industrial plants are exemplified in Figure 2-5, for production stop in the range of 500-4000 hours and under specific assumptions on the loss of profit. During the production stop, fixed running costs and annualized capital $\operatorname{costs}^{16}$ are accounted for together with the loss in profit (for example with a $20 \%$ profit margin on the product cost). From it is evident that a lengthy production stop for retrofitting CCS has a significant economic impact and will add considerably to $\mathrm{CO}_{2}$ avoidance costs, especially if only a small part of the production stop can be aligned with a routinely scheduled production stop for maintenance. This can be exemplified in retrofitting a cement plant with oxyfuel technology, where, under specific assumptions, a 4000-hours production stop for significant modifications to be implemented in the core process would increase the $\mathrm{CO}_{2}$ avoidance cost by over $15 \%$, assuming a $20 \%$ profit margin on the product. In the case of retrofitting a pulp mill's recovery boiler with amine $\mathrm{CO}_{2}$ capture, a shorter production stop of 1000 hours might suffice for the end-of-pipe technology, resulting in around $6 \%$ increase in the $\mathrm{CO}_{2}$ avoidance cost.

In addition to the length of the production stop, the product profit margin will also impact the added cost of $\mathrm{CO}_{2}$ avoided, as exemplified in Figure 2-5, for a production stop of 500, 1000, and $4000 \mathrm{~h}$ in a cement plant, pulp mill, and an integrated steel mill.

Table 2-3: Examples of key data for three different industrial plants.

\begin{tabular}{|l|c|c|c|}
\hline Plant type - product & $\begin{array}{c}\text { Cement plant } \\
(\text { CEMCAP [80]) } \\
- \text { clinker }\end{array}$ & $\begin{array}{c}\text { Pulp mill } \\
\text { (IEAGHG [82]) } \\
- \text { air-dried pulp }\end{array}$ & $\begin{array}{c}\text { Integrated steel mill } \\
\text { (IEAGHG [78]) - } \\
\text { hot rolled coil }\end{array}$ \\
\hline Production rate [tonne of product/hour] & 120 & 95 & 500 \\
\hline Production cost without CCS & $63 €_{2014} / \mathrm{t}_{\text {clinker }}$ & $523 €_{2015} /$ adt $^{\mathrm{a}}$ & $451 €_{2014} / \mathrm{t}_{\mathrm{HRC}} \mathrm{a}^{\mathrm{a}, \mathrm{b}}$ \\
\hline $\mathrm{CAPEX}$ and fixed OPEX of plant & $39 €_{2014} / \mathrm{t}_{\mathrm{clinker}}$ & $248 €_{2015} / \mathrm{adt}^{\mathrm{a}}$ & $204 €_{2014} / \mathrm{t}_{\mathrm{HRC}}$, \\
\hline $\mathrm{CO}_{2}$ emissions in normal operation w/o $\mathrm{CO}_{2}$ capture $\left[\mathrm{tCO}_{2} / \mathrm{h}\right]$ & 103 & 257 & 1047 \\
\hline
\end{tabular}

${ }^{\mathrm{a}}$ adt and hrc stand for air-dried ton and hot-rolled coil, respectively.

${ }^{b}$ Costs converted from $\$ 2010$ to $€ 2014$ using the Chemical Engineering Cost Plant Index (CEPCI) and average currency exchange rates.

\footnotetext{
${ }^{16}$ Repayment of investment and operating costs such as labour, maintenance, etc.
} 


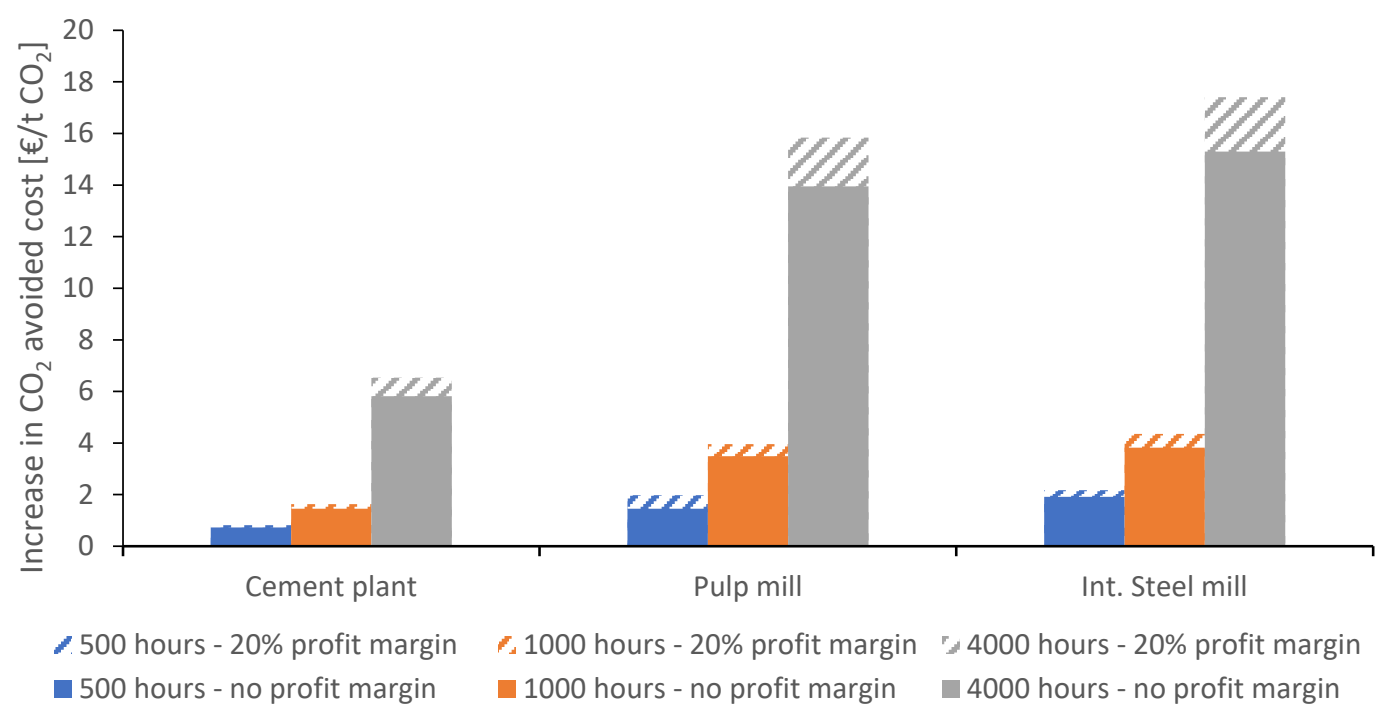

Figure 2-5. Increase in $\mathrm{CO}_{2}$ avoided cost, as a result of a whole-plant production stop, for production stop durations from 500 to $\mathbf{4 0 0 0}$ hours, and with profit margin losses on the product cost during production stop of 0 and $20 \%$.

Figure footnote: During the production stop, fixed running costs and annualized capital costs are accounted for together with the loss in profit. The increase in $\mathrm{CO}_{2}$ avoided cost is calculated by dividing these costs and profit loss during the plant

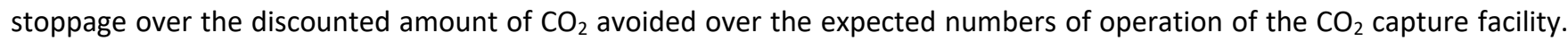
Here, 25 years of operation and a real discount rate of $8 \%$ are considered. It should be noted that for simplification, the amount of $\mathrm{CO}_{2}$ avoided is calculated with a $90 \% \mathrm{CO}_{2}$ capture rate, $90 \%$ capacity factor of the industrial plant, and does not account for emissions originating from potential increase in energy demand of the industrial plant after $\mathrm{CO}_{2} \mathrm{capture}$ is implemented.

\subsubsection{Impacts on the product quality and plant operation}

The integration of $\mathrm{CO}_{2}$ capture technologies can also have an impact on the main output product(s) of the plant. For example, if no other process modifications are implemented, $\mathrm{CO}_{2}$ oxyfuel combustion in a cement plant has a direct impact on the temperature in the cement kiln, which can negatively affect the clinker phase formation and consequently the product quality [107]. Another example is oxyfuel combustion in the blast furnace of an integrated steel mill. In this case, $\mathrm{H}_{2}$ and $\mathrm{CO}$ rich flue gases from the furnace are partly recycled, after the $\mathrm{CO}_{2}$ has been separated from the gases, to regulate the mass and energy balance of the furnace. This drastically different atmosphere in the furnace can affect the product to some extent but will also have a large impact on the energy balance of the whole steel mill which could make this technology difficult to implement in existing plants [108].

Implementation of capture technologies that affect the core plant's main product may result in additional costs for modifications of the original process and equipment, post-treatment to meet existing product standards, or a decrease in product value. In such cases, both should be attributed to the cost of $\mathrm{CO}_{2}$ capture. A lower product value will most likely yield lower revenues. These situations are more likely to occur in retrofit applications, where changes to the primary process may be less feasible than in new plant applications. Conceivably, however, technology innovations and process modifications to accommodate the new carbon constraint may also result in net benefits and cost savings (as occurred, for example, when CFCs were banned from certain industrial processes).

In practice, it can be challenging to assess foregone revenues (or additional profits) since the exact market value (product price) is often unknown. If a sound assessment is not possible, practitioners of costing studies should at least clearly report the assumed cost effect and consider sensitivity analyses. In general, the required product quality is dictated by consumers' demands. For that reason, maintaining the product quality will often have priority for the plant operator. 


\subsubsection{Spatial constraints for $\mathrm{CO}_{2}$ capture equipment in existing industrial plants}

Space restrictions or safety considerations on industrial sites could severely affect the technical and economic feasibility of installing $\mathrm{CO}_{2}$ capture equipment and their supporting utilities on industrial sites. Unlike new-build (greenfield) plants with CCS, existing (brownfield) facilities were not designed to accommodate spacious capture equipment, thus possibly making retrofit applications of CCS more challenging and costly. Although spatial constraints vary considerably on a case-by-case basis, depending on the design and layout of the plant as well as on the capture technology, infrastructural modifications (e.g. flue gas re-routing and sub-optimal unit location) and replacement of existing installations on the plant site, may be required [93, 109].

In most cases, spatial constraints can be solved by placing the $\mathrm{CO}_{2}$ capture unit, or part of it, further away from the emission point source. However, this implies flue gas transport over longer distances, requiring large-diameter and expensive stainless-steel ducting, and possibly modifications to the existing industrial plant. In addition, the transport of the gaseous flue gas through the ducting system might be very energyintensive and thus costly and may even require additional equipment (e.g. blowers). In some cases, alternative capture configurations in which only part of the capture unit is placed near the emission point source can provide a workable solution. For example, in some cases, it could be more cost-efficient to locate only the absorption section of an amine-based capture process near the $\mathrm{CO}_{2}$ emission point and transport the $\mathrm{CO}_{2}$ absorbed in the rich solvent to the regeneration and $\mathrm{CO}_{2}$ compression section located further away [93, 110]. In addition, capture configurations can even span multiple industrial plants whereby capture components, such as solvent regenerators and compressors, may be shared. Such configurations do not only circumvent spatial limitations on individual plant sites, but may also offer the possibility to curtail average capture costs by exploiting economies of scale compared to a set of individual plant chains [111].

Figure 2-6 illustrates six stylised examples of layout alternatives that may be considered depending on potential spatial constraints for a solvent-based $\mathrm{CO}_{2}$ capture process. Case (a) represents the scenario in which there is enough space near the flue gas point source to accommodate the absorber, desorber, and compression sections of the capture process. This case tends to be more cost-effective when considering $\mathrm{CO}_{2}$ capture from a single $\mathrm{CO}_{2}$ point source as it minimises the high cost associated with the flue gas ducting and rich/lean solvent transport. Case (f) represents the other extreme in which both the absorber and desorber sections are placed far away from the flue gas point source due to spatial constraints. This option tends to result in significantly higher cost than case (a) due to the large flue gas ducting required. Meanwhile, cases (b) to (d) represent hybrid configurations. For example, in case (b), there is enough space near the flue gas point source for the absorption section but not for the desorber section. As a result of this, the desorber may be placed further away from the flue gas point source and the $\mathrm{CO}_{2}$ would be transported as a $\mathrm{CO}_{2}$-rich solvent between the two sections of the $\mathrm{CO}_{2}$ capture process. Case (c) is similar to case (b) with the exception that it considers that the spatial constraint would lead to flue gas ducting to reach an area with sufficient space for the absorber section. Finally, cases (d) and (e) illustrate that further complexity may arise when considering $\mathrm{CO}_{2}$ capture from multiple sources, with potentially different $\mathrm{CO}_{2}$ concentrations and impurities, within the plant which may be the case for example for refineries, iron and steel mills, etc. 


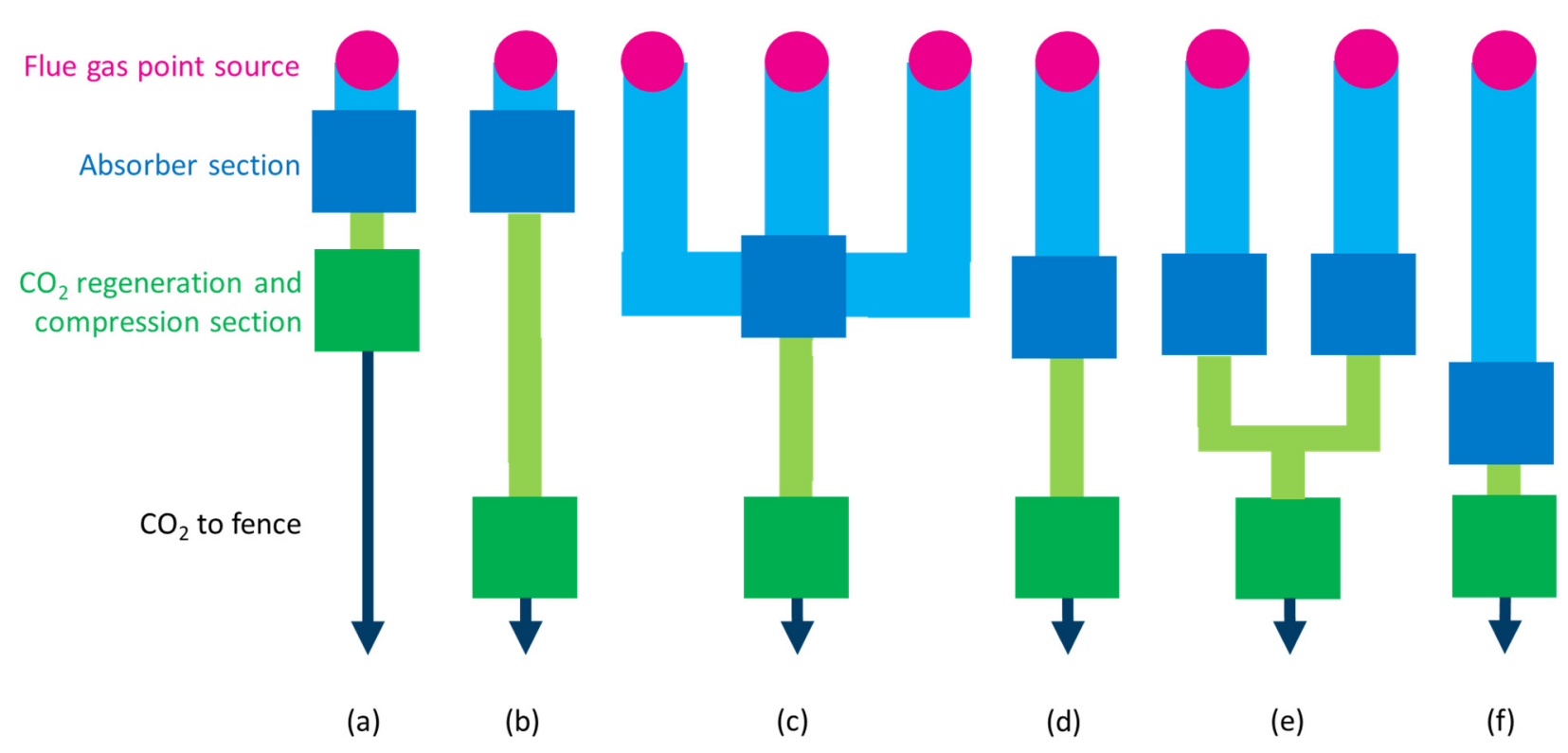

Figure 2-6. Illustration of different layout alternatives that could be considered in space-constraint cases.

In general, the additional costs resulting from spatial constraints are not always considered, outside of detailed engineering studies, when discussing costs of CCS in industry. Although this can be a reasonable assumption when considering greenfield development of the industrial plant with CCS [112,113], in other cases this may not be justified as these costs may increase the cost of $\mathrm{CO}_{2}$ capture and conditioning in a non-negligible way. For example, in the case of $\mathrm{CO}_{2}$ capture retrofit to a refinery, the flue gas and utilities

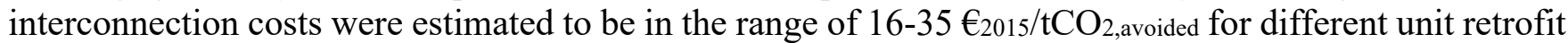
scenarios [93]. Furthermore, as space requirements for the $\mathrm{CO}_{2}$ capture unit is technology-specific, spatial constraints may significantly benefit compact and modular capture technologies, as they could avoid significant flue gas interconnection costs [106], thus impacting the capture technology selection.

Although the impact of spatial constraints and flue gas interconnection costs ought to be considered in techno-economic studies on $\mathrm{CO}_{2}$ capture retrofit from industrial plants, it is important to realise that these costs are very much site and $\mathrm{CO}_{2}$ capture technology-specific. These costs shall thus be based on the evaluation of the layout of the industrial site with the considered $\mathrm{CO}_{2}$ capture technology. In order to better help accounting for these costs, an example of the direct costs associated with the installation of a pipeline rack with a flue gas duct are illustrated in Figure 2-7 as a function of the exhaust flue gas flowrate for different transport distances (d).

Finally, the costs associated with utilities ${ }^{17}$ production and their integration with the $\mathrm{CO}_{2}$ capture unit can also be impacted by space constraints. In some cases, the $\mathrm{CO}_{2}$ capture unit may be located at significant distances from relevant utilities production and treatment facilities. This may happen in cases in which new utilities production and treatment facilities could not be placed close to the $\mathrm{CO}_{2}$ capture unit due to space constraints. However, this may also be the case when existing utilities production and treatment facilities with spare capacity are integrated with the $\mathrm{CO}_{2}$ capture unit in order to reduce costs and/or investment. Although these are rarely included in cost evaluations, these costs can be non-negligible in retrofit cases depending on the overall layout of the industrial plant with the $\mathrm{CO}_{2}$ capture unit and its associated utilities. Thus, these costs must be included to make more realistic cost estimates as well as to better understand the complete impact of certain design decisions such as the use of existing spare capacities.

\footnotetext{
${ }^{17}$ Power, heat, cooling water, process water, etc.
} 


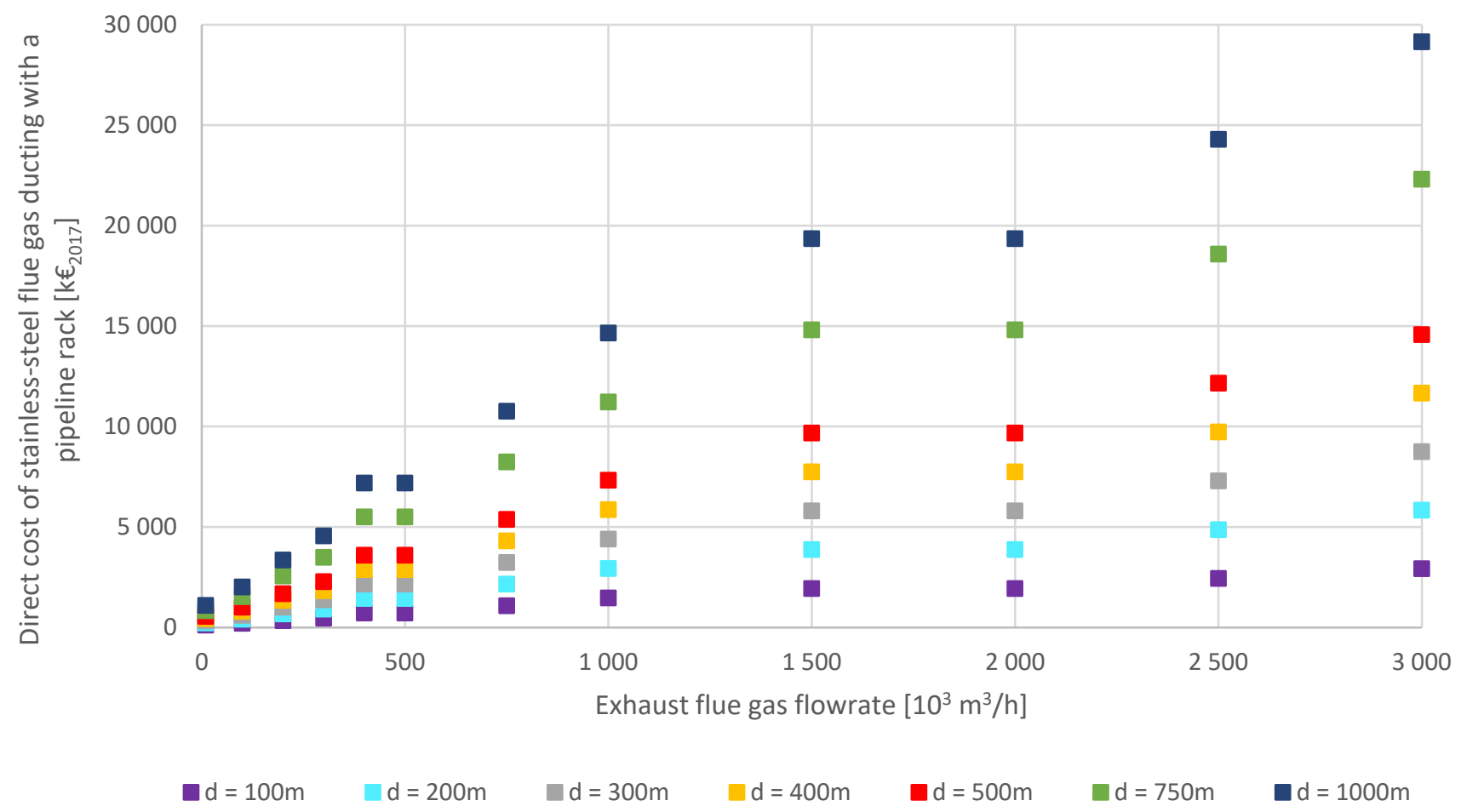

Figure 2-7. Direct cost associated with exhaust flue gas ducting in function of the exhaust flue gas flowrate for different transport distances (d)

Figure footnote: These costs, estimated with Aspen Process Economic Analyzer [114], correspond to direct costs associated with the installation of a pipeline rack with a flue gas duct in stainless steel $304^{18}$ for a Dutch-based location.

\subsubsection{Flue-gas treatment requirements}

Another element which can have a significant impact on the costs of $\mathrm{CO}_{2}$ capture from industrial plant is the presence of impurities in the flue gas to be treated in the $\mathrm{CO}_{2}$ capture process. Indeed, industrial flue gases can contain levels of impurities which may impact the performances and design of the $\mathrm{CO}_{2}$ capture and downstream CCS system. For example, the presence of $\mathrm{SO}_{\mathrm{x}}$ and $\mathrm{NO}_{\mathrm{x}}$ can lead to significant solvent degradation in an amine-based $\mathrm{CO}_{2}$ capture thus resulting in poorer capture levels, higher energy penalties, and a more costly process. Similarly, certain membranes can be very sensitive to the presence of water while others are not. Depending on type and level of impurities of the plant flue gas and the technologies considered along the CCS chain, pre-treatment might thus be required prior or downstream of the $\mathrm{CO}_{2}$ capture process to reach satisfactory levels of impurities. This could for example mean desulphurisation (DeSOx), NOx removal, oxygen removal, dehydration, dust removal, etc. The cost associated with this or these potential treatment(s) may have a significant impact on capture costs, the comparison of capture technologies [115], as well on the performances and technology selection in the transport and storage part of the chain $[116,117]$. Cost studies that ignore these additional costs or fail to attribute them to the cost of CCS might understate the real costs of CCS.

Typical ranges of flue gas $\mathrm{CO}_{2}$ content and levels of $\mathrm{SO}, \mathrm{NO}$, and dust in different industry flue gases are summarised in Table 2-4.

\footnotetext{
${ }^{18}$ Stainless steel 304 is here considered due to the expected presence of water in the flue gas. It is worth noting that the material selection may differ depending on water level, as well type and level of other corrosive impurities.
} 
Table 2-4: Typical $\mathrm{CO}_{2}$ concentrations and impurities in flue gas prior to $\mathrm{CO}_{2}$ capture in various energy-intensive industries. Note that other impurities than listed here might be present as well.

\begin{tabular}{|c|c|c|c|c|c|}
\hline Industry & $\begin{array}{l}\mathrm{CO}_{2} \text { source } \\
\text { within facility }\end{array}$ & $\begin{array}{l}\mathrm{CO}_{2} \text { concentration } \\
(\% \mathrm{vol})\end{array}$ & SOx & NOx & $\begin{array}{l}\text { Particulate } \\
\text { matter }\end{array}$ \\
\hline $\begin{array}{l}\text { Cement }[79,106, \\
115]\end{array}$ & Cement kiln stack & $18-22$ & $\begin{array}{l}10-3500 \\
\mathrm{mg} / \mathrm{Nm}^{3}\end{array}$ & $\begin{array}{l}200-3000 \\
\mathrm{mg} / \mathrm{Nm}^{3}\end{array}$ & $5-200 \mathrm{mg} / \mathrm{Nm}^{3}$ \\
\hline $\begin{array}{l}\text { Iron and steel [78, } \\
108,118]\end{array}$ & $\begin{array}{l}\text { Power station } \\
\text { Blast furnace } \\
\text { Other stacks }\end{array}$ & $\begin{array}{l}25-30 \\
25 \\
14-25 \\
\end{array}$ & $\begin{array}{l}10-20 \mathrm{mg} / \mathrm{Nm}^{3} \\
10 \mathrm{mg} / \mathrm{Nm}^{3} \\
10-300 \mathrm{mg} / \mathrm{Nm}^{3}\end{array}$ & $\begin{array}{l}50-60 \mathrm{mg} / \mathrm{Nm}^{3} \\
60 \mathrm{mg} / \mathrm{Nm}^{3} \\
30-500 \mathrm{mg} / \mathrm{Nm}^{3}\end{array}$ & $\begin{array}{l}<5 \mathrm{mg} / \mathrm{Nm}^{3} \\
<5 \mathrm{mg} / \mathrm{Nm}^{3} \\
<5 \mathrm{mg} / \mathrm{Nm}^{3}\end{array}$ \\
\hline $\begin{array}{l}\text { Oil refining } \quad[86, \\
93,119]\end{array}$ & $\begin{array}{ll}\text { Fluid } & \text { Catalytic } \\
\text { Cracker } & \\
\text { Process } & \text { heaters } \\
\text { stacks* } & \end{array}$ & $\begin{array}{l}14-17 \\
8-14\end{array}$ & $\begin{array}{l}700-800 \mathrm{mg} / \mathrm{Nm}^{3} \\
5-350 \mathrm{mg} / \mathrm{Nm}^{3}\end{array}$ & $80-350 \mathrm{mg} / \mathrm{Nm}^{3}$ & $\begin{array}{l}- \\
- \\
-\end{array}$ \\
\hline $\begin{array}{l}\mathrm{H}_{2} \text { production [86, } \\
93]\end{array}$ & $\begin{array}{l}\text { Steam methane } \\
\text { reformer }\end{array}$ & $20-25$ & $60 \mathrm{mg} / \mathrm{Nm}^{3}$ & $120-150 \mathrm{mg} / \mathrm{Nm}^{3}$ & - \\
\hline Pulp and paper [82] & Recovery boiler & 13 & $1-100 \mathrm{mg} / \mathrm{Nm}^{3}$ & $120-250 \mathrm{mg} / \mathrm{Nm}^{3}$ & $5-190 \mathrm{mg} / \mathrm{Nm}^{3}$ \\
\hline
\end{tabular}

* Flue gases from different process heaters vented through the same stack

To illustrate the impact additional flue gas treatment can have on capture cost, additional costs for DeSOx pre-treatment are exemplified in Figure 2-8, for different cement and oil refinery flue gases [80, 93, 106]. The presence of DeSOx treatment in industrial plants is indeed highly industry- and site-specific and depends on the nature of the core production process, the characteristics of fuels used in the process as well as local environmental legislations. Industrial plants might fulfil SOx emission standards without a DeSOx system, but when it comes to implementing $\mathrm{CO}_{2}$ capture, some post-combustion systems might require reduction of SOx to lower levels to minimize detrimental effects on the capture process performance. In that sense, there is an economic trade-off between the extent of additional flue gas purification and less deteriorating effects on capture process performance.

Considering treatment requirements downstream of the $\mathrm{CO}_{2}$ capture unit, the high purity $\mathrm{CO}_{2}$ stream from the $\mathrm{CO}_{2}$ capture system needs to reach the quality requirements of the transportation system or the $\mathrm{CO}_{2}$ utilisation process. Similarly, the cost linked to that post-treatment needs to be considered in the whole CCUS system evaluation.

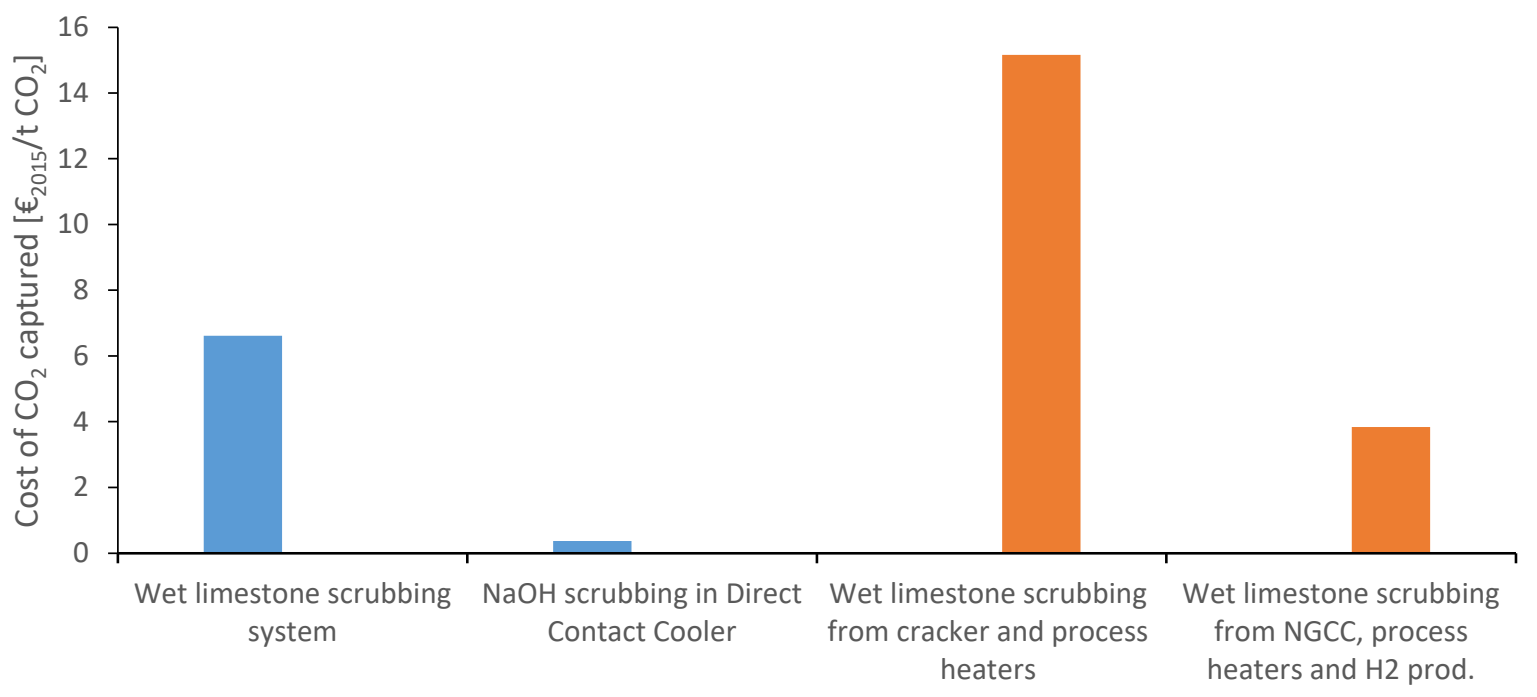

DeSOX for MEA absorption in cement plant a DeSOX for MEA absorption from refinery flue gases

Figure 2-8. Cost of flue gas desulphurisation $\left[€_{2015} / \mathrm{t} \mathrm{CO}\right.$ captured], exemplified for MEA absorption applied on cement plant and refinery flue gases.

Figure footnote: Based on data extracted from the studies on $\mathrm{CO}_{2}$ capture from a cement production facility [80] two flue gases from a refinery [93]. 


\subsection{3 $\mathrm{CO}_{2}$ transport and storage costs}

Several studies have discussed the costs of $\mathrm{CO}_{2}$ transport and storage in detail [120-122]. In practice, it is important to note that these costs are influenced by a variety of factors: transport mode (e.g. pipeline, ship), flow rate, transport distance, spatial configuration of transport system, type of storage (e.g. saline aquifer, depleted gas field), and characteristics of storage site (e.g. storage capacity, permeability, porosity).

This section discusses key cost aspects related to transport and storage of $\mathrm{CO}_{2}$ captured from industrial point sources. It is worth noting that estimates presented in the section exclude the $\mathrm{CO}_{2}$ conditioning (purification and compression) costs at the capture facilities unless otherwise indicated.

\subsubsection{Impact of the amount of $\mathrm{CO}_{2}$ captured on transport and storage cost}

Industrial plants vary considerably in terms of annual $\mathrm{CO}_{2}$ emissions, typically ranging from 0.15 $\mathrm{MtCO}_{2} / \mathrm{y}$ to $14 \mathrm{MtCO}_{2} / \mathrm{y}$ depending on size and type of the individual plant (see Table 2-1). Consequently, the amount of $\mathrm{CO}_{2}$ captured, and thus transported and stored, can vary significantly. In addition, plant operators may decide to capture only a share of the plant's $\mathrm{CO}_{2}$ emissions, either because it is physically impossible to capture all $\mathrm{CO}_{2}$ emissions due to spatial constraints (see Section 2.3.2.3), or because of economic reasons. Many industrial plants have multiple point sources with different characteristics in terms of waste heat availability, spatial constraints, and $\mathrm{CO}_{2}$ volume and concentration, resulting in varying $\mathrm{CO}_{2}$ capture costs. As a result, plant operators may only capture $\mathrm{CO}_{2}$ from the point sources with the lowest cost, depending on the policy framework $[93,123]$.

The cost of $\mathrm{CO}_{2}$ transport and storage is strongly linked to the $\mathrm{CO}_{2}$ flow rate, which in turn is a function of the $\mathrm{CO}_{2}$ capture rate at the industrial plant. As a result, transport and storage costs may differ significantly from one industrial plant to another and make up a considerably larger share in the total CCUS chain cost for smaller emitters. Nevertheless, many literature studies assume a fixed cost for $\mathrm{CO}_{2}$ transport and storage (often $10 € / \mathrm{tCO}_{2}$ ) regardless of their considered $\mathrm{CO}_{2}$ flow rate [21].

Figure 2-9 to Figure 2-11 illustrate costs of transport and storage per tonne of $\mathrm{CO}_{2}$ for the Northwest European region. These estimates illustrate how costs of $\mathrm{CO}_{2}$ transport and storages can sharply decrease with higher $\mathrm{CO}_{2}$ flow rates due to economies of scale, and hence why an assumed fixed $\mathrm{CO}_{2}$ transport and storage cost independent of the considered flow, distance to storage and storage characteristics may not be appropriate. While the estimates presented in Figure 2-9 to Figure 2-11 could already support better CCS estimates more representative transport and storage costs, case-specific evaluations also reflecting cost specific to the considered region are recommended.

It is also important to note that pooling demand for transport and storage capacity by sharing pipeline and storage infrastructures can significantly reduce the average unitary cost, which might be particularly beneficial for small emitters. For example, for a transport distance of $250 \mathrm{~km}$ via onshore pipeline, increasing the annual transport flow rate from 0.5 to $5 \mathrm{MtCO}_{2} / \mathrm{y}$ would reduce average transport cost more than three times, from over $20 €_{2017} / \mathrm{tCO}_{2}$ to around $6 €_{2017} / \mathrm{tCO}_{2}$ (see Figure 2-9). While a shared infrastructure may have lower total system costs than a set of stand-alone solutions, it does involve several challenges, including higher upfront investments as well as coordination and cost allocation among the different users. 


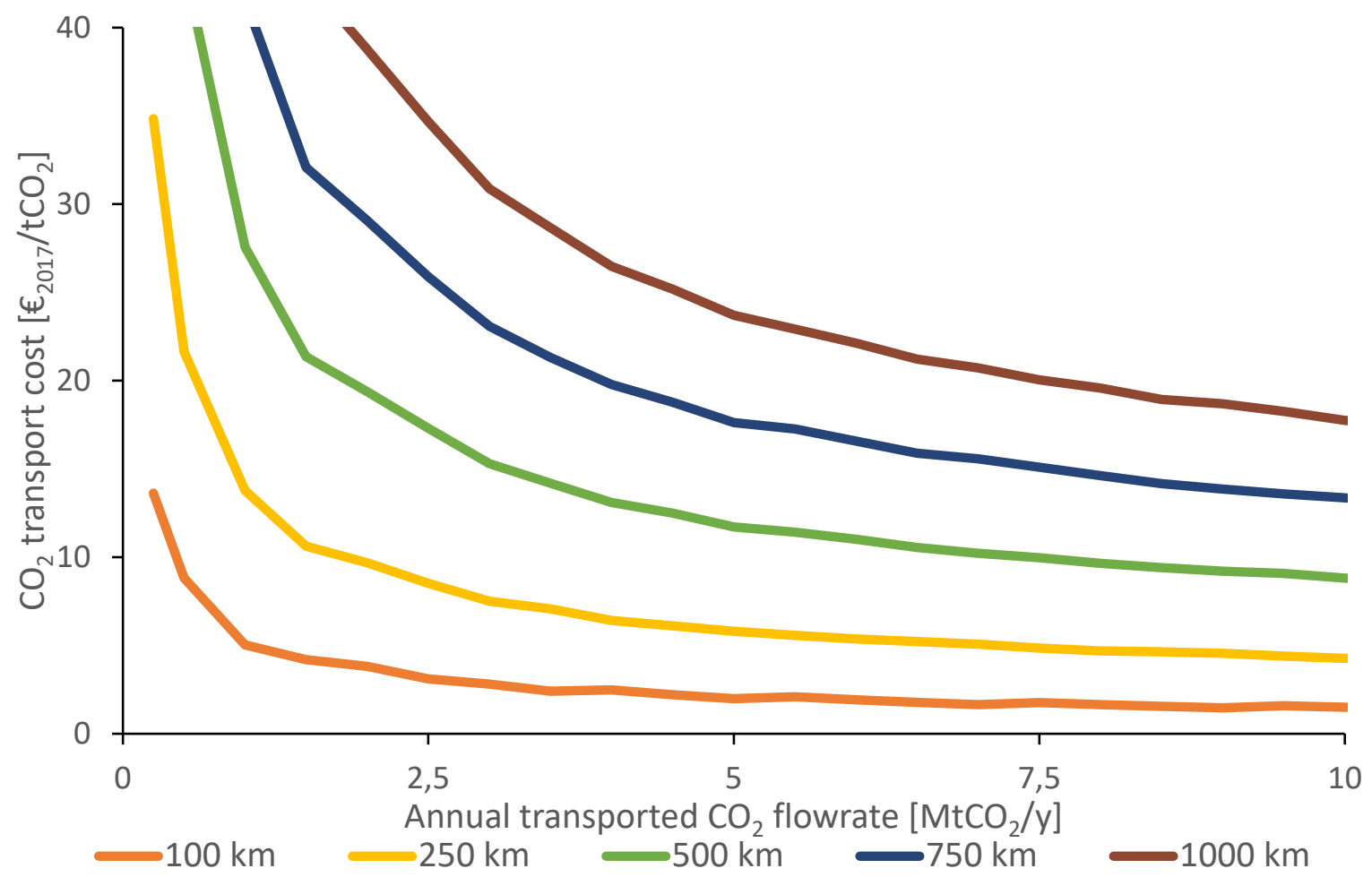

Figure 2-9. $\mathrm{CO}_{2}$ transport cost via onshore pipeline in function of the annual transported $\mathrm{CO}_{2}$ flowrate for different transport distances

Figure footnote: Established using the iCCS tool [124, 125] and considering the pipeline cost model from Knoope et al. [126]. While the $\mathrm{CO}_{2}$ is assumed to be available at 110 bar after $\mathrm{CO}_{2}$ conditioning, the pipeline diameter and number of reboosting station(s) is optimised for each combination of transport flowrate and distance. These estimates are based on a project duration of 25 years and a real discount rate of $8 \%$.

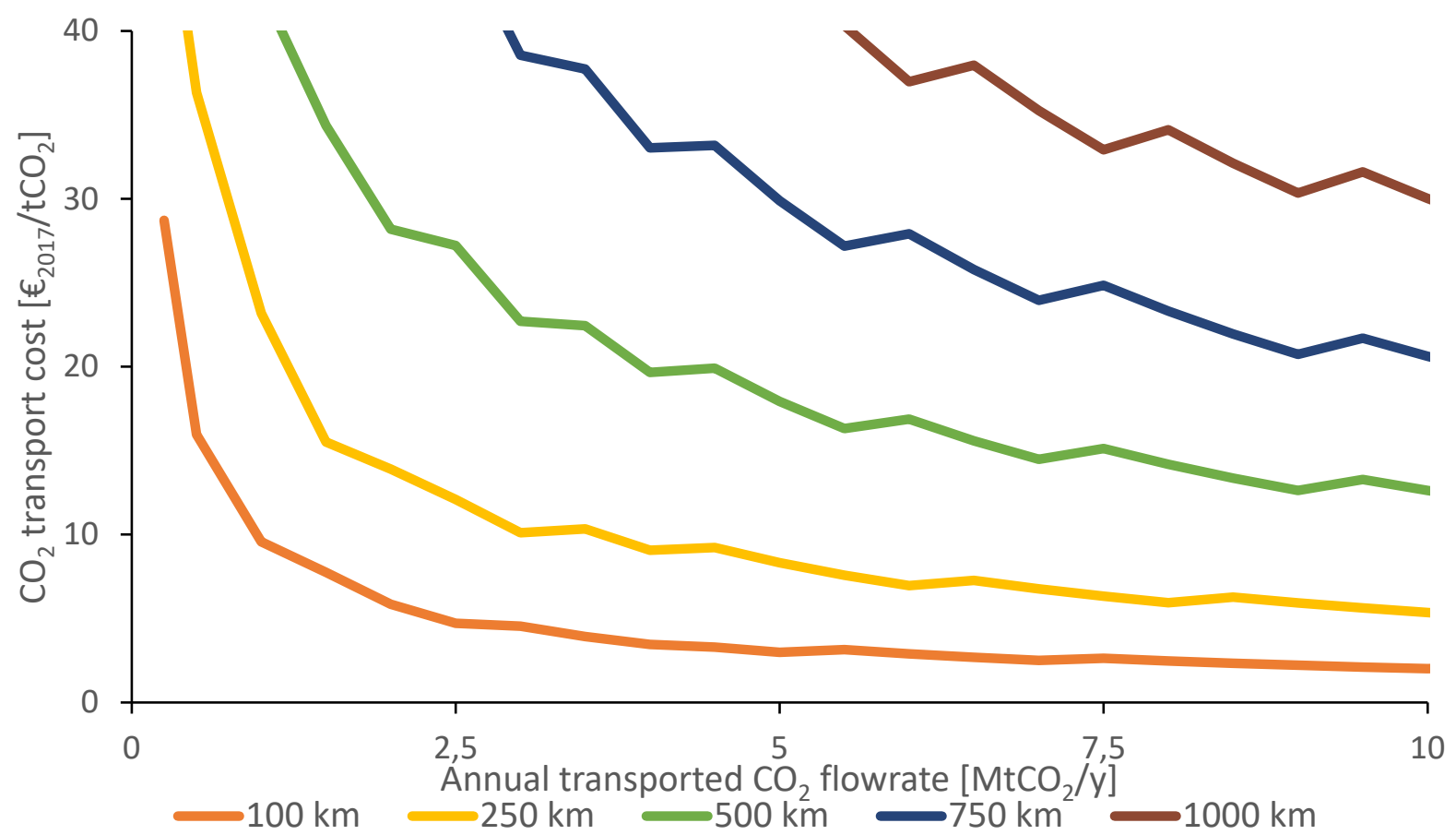

Figure 2-10. $\mathrm{CO}_{2}$ transport cost via offshore pipeline in function of the annual transported $\mathrm{CO}_{2}$ flowrate for different transport distances

Figure footnote: Established on the same basis as Figure 2-9 although no offshore reboosting station is considered due to prohibitive cost. 


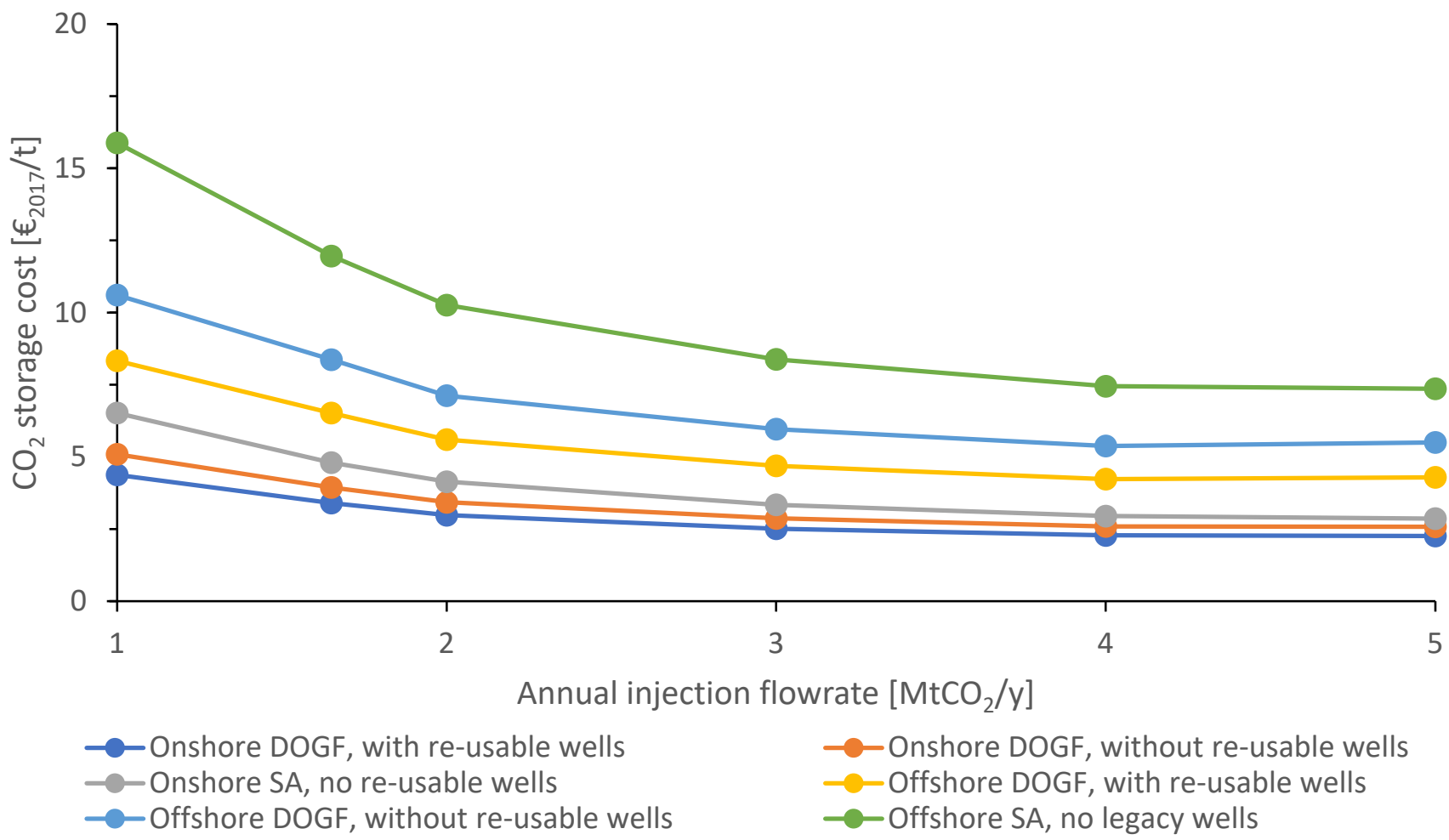

Figure 2-11. $\mathrm{CO}_{2}$ storage cost in function of the annual injection flowrate for different types of storage scenario (DOGF: Depleted Oil and Gas Field, SA: Saline Aquifer)

Figure footnote: Established using the iCCS tool $[91,127]$ based on the $\mathrm{CO}_{2}$ storage cost methodology established by the ZEP [121]. The cost of an onshore new well is assumed to be 7,3 $\mathrm{M}_{2017}$, while other costs were updated using the IHS Upstream Costs Index [128]. These estimates are based on a project duration of 40 years and a real discount rate of $8 \%$.

In addition to evaluation of CCS from industry based on pipeline transport, it is worth noting that transport of $\mathrm{CO}_{2}$ via ship, and more generally tanked-based solutions (ship, barge, train, truck) is more and more considered for CCS from industry, especially in Europe [129]. Indeed, while pipeline transport has traditionally been the default option considered in CCS-based evaluations, ship-based transport of $\mathrm{CO}_{2}$ can be an attractive option for industrial emitters in some cases, due to its cost efficiency for small $\mathrm{CO}_{2}$ volumes and transport over long distances [125, 130]. Furthermore, shipping typically involves lower upfront investments, shorter construction time, offers more flexibility, could be easier in terms of environmental permitting, and may present opportunities for co-utilisation of infrastructures [131].

Figure 2-12 and Figure 2-13 illustrate the cost of $\mathrm{CO}_{2}$ transport by ship together with pipeline transport costs to indicate when shipping is cheaper than pipeline-based transport. To ensure a fair comparison between pipeline and ship means of transportation, the cost estimates for ship-based $\mathrm{CO}_{2}$ transport also include the increase in conditioning cost compared to a pipeline-based transport. As illustrated, it is worth noting that the cost of ship-based transport is less affected by the annual flowrate and transport distances than pipeline transport. Shipping transport can be the preferred means of transport for a wide range of transport distances especially for small annual flowrates. For example, shipping between harbours would be the cost-optimal option for distances above $250 \mathrm{~km}$ when transporting an annual flowrate of $1 \mathrm{MtCO}_{2} / \mathrm{y}$, while for higher annual flow rates pipeline transport is more cost-efficient for a wider range of transport distances. While the estimates presented in Figure 2-12 and Figure 2-13 can be used to support better CCS estimates of ship-based chains, more details on when pipeline and shipping transport are most efficient can be found in literature $[125,130]$. 


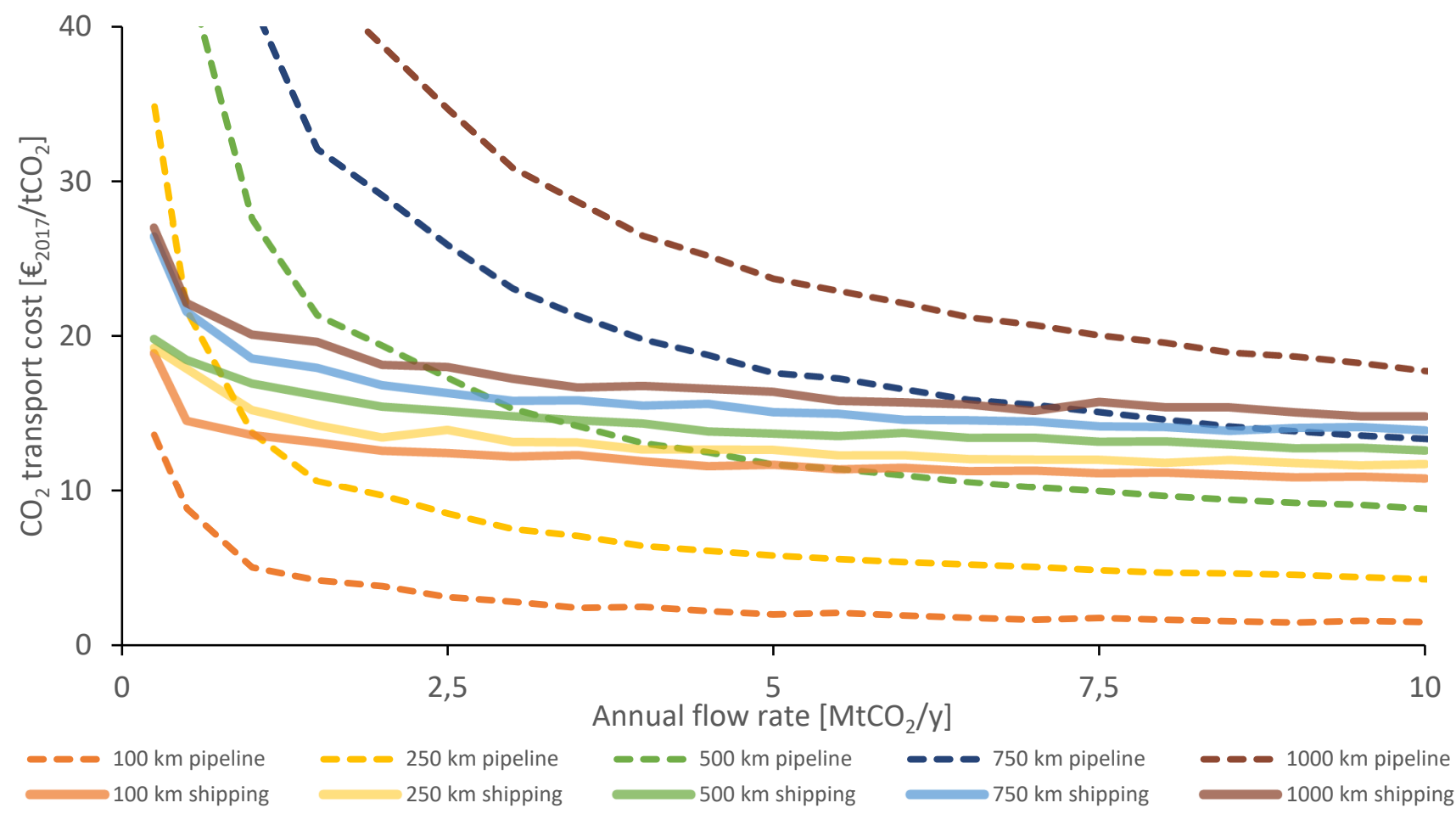

Figure 2-12. $\mathrm{CO}_{2}$ conditioning and transport cost via shipping between harbours in function of the annual transported $\mathrm{CO}_{2}$ flowrate for different transport distances

Figure footnote: Established using the iCCS tool $[124,125]$ and considering ship costs from Durusut and Joos $[132]$. The $\mathrm{CO}_{2}$ is considered to be transported at $7 \mathrm{barg}$, while the optimal number of ships and ship capacity are optimised for each combination of transport flowrate and distance. While the whole cost of conditioning is not included, the increase in conditioning cost compared to pipeline transport is included to ensure a fair comparison between shipping and pipeline transport costs. These estimates are based on a project duration of 25 years and a real discount rate of $8 \%$.

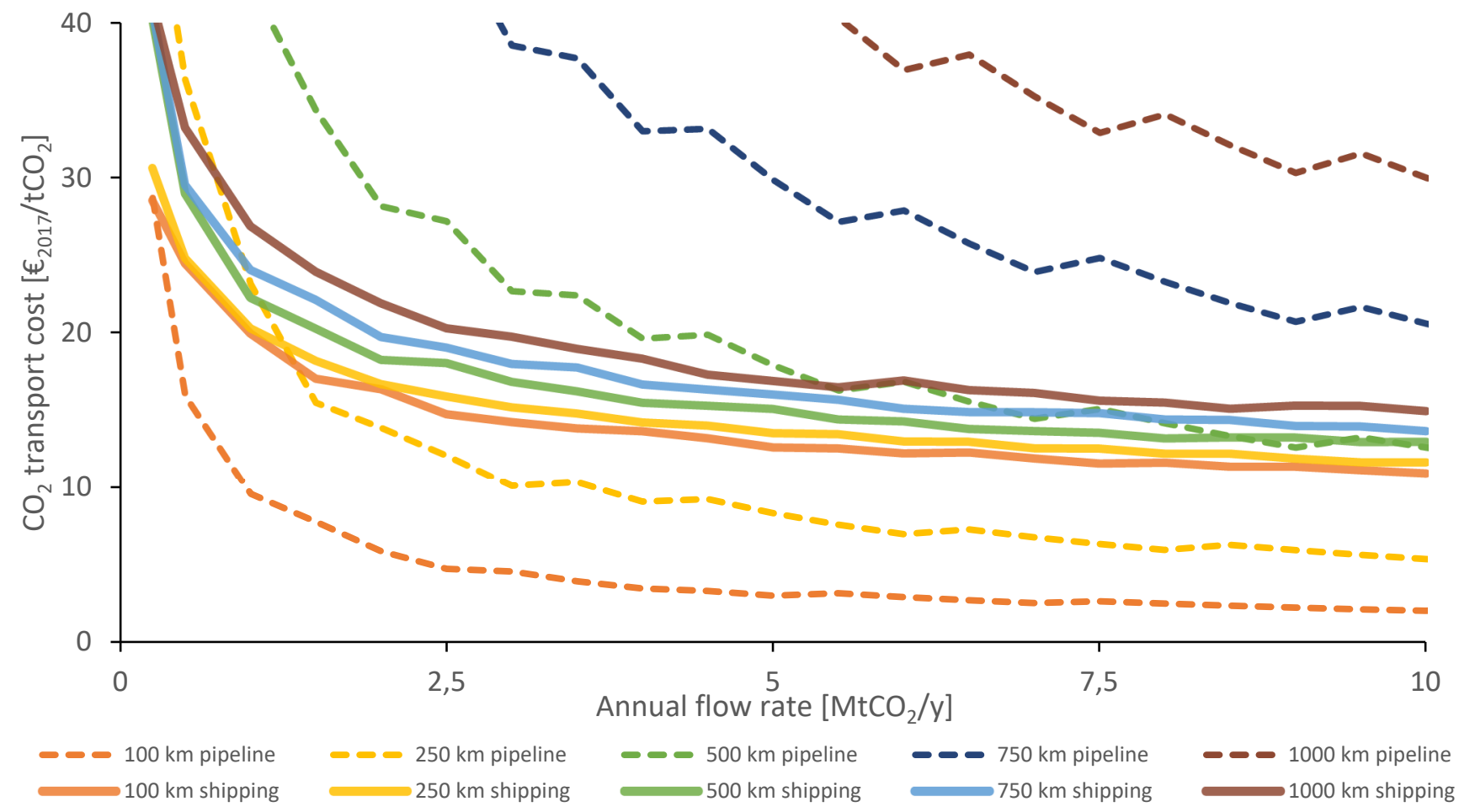

Figure 2-13. $\mathrm{CO}_{2}$ conditioning and transport cost via shipping to an offshore site in function of the annual transported $\mathrm{CO}_{2}$ flowrate for different transport distances

Figure footnote: Established on the same basis as Figure 2-12. 


\subsubsection{Other elements of potential importance}

In addition to the issues discussed before, it is worth paying attention to the following elements when assessing the cost of $\mathrm{CO}_{2}$ transport and storage from industrial emitters:

- Installing pipelines in cramped industrial areas can be costly and time-consuming to construct. In dense industrial sites, excavation work may have to be carried out manually to reduce the risk of damaging other pipelines. Similarly, studies have shown that limited space availability in underground communal pipeline corridors could results in several detours and/or higher operating pressures necessary, which increase local transport costs and possibly prolong license procedures [133].

- Even though high purity $\mathrm{CO}_{2}(>95 \%)$ is normally targeted after $\mathrm{CO}_{2}$ capture, different types and levels of impurities may be present in the $\mathrm{CO}_{2}$ to be transported and stored. Although these impurities may only be present in small amounts, several studies have shown that the potential associated impurities can have a significant impact on design and cost of $\mathrm{CO}_{2}$ conditioning, transport, and storage $[116,117,134,135]$. However, this impact will however depend on the types and levels of impurities present in the $\mathrm{CO}_{2}$ stream and thus the combination of industrial plant, $\mathrm{CO}_{2}$ capture technology considered and targeted $\mathrm{CO}_{2}$ specifications for storage or use [136]. Finally, it is worth noting that there are still some knowledge gaps in term of impact of multicomponent impurities on underlying aspects influencing costs of such systems: thermodynamic behaviour, physical properties, corrosion, etc.

- The cost for $\mathrm{CO}_{2}$ transport and storage can vary significantly by country and region, depending on local costs for rights-of-way, labour, materials and other inputs [137]. Study authors should ensure that cost models representative of the considered geographical region are used. Such models for onshore pipeline transport have been developed by, for example, McCoy [137], Knoope et al. [126], and Wei et al. [138] for the U.S., Northwest Europe and China.

- It is also worth noting that external political and social aspects may also have an impact on transport and storage costs. For example, onshore $\mathrm{CO}_{2}$ storage has been prohibited in several European countries thus leading in practice to more expensive transport routes and offshore storage [139]. Furthermore, potential social acceptance issues may also impact the routing of the $\mathrm{CO}_{2}$ transport thus resulting in higher costs. While CCS evaluations shall aim to represent expected conditions taking also these aspects into account, political and social aspects can result in uncertainties which may impact both design and costs.

- Finally, it is worth noting that there could be potential trade-offs between transporting $\mathrm{CO}_{2}$ and transporting products and raw materials of an industrial plant. In some cases, it could be that $\mathrm{CO}_{2}$ management costs would change the preferred location of a new industrial plant $[85,140]$.

\subsection{Transferability of experience and technology maturity from power to industry sectors}

Technology maturity is an important factor in cost estimates, usually accounted for through process contingency costs as illustrated in Chapter 1 and previously by other organisations like AACE and EPRI $[141,142]$. Maturing and operational experience of $\mathrm{CO}_{2}$ capture technologies has to date been gained primarily through decades of industrial applications in the chemical and petrochemical industries, for processes such as natural gas processing, as well as in small-scale applications at power plants to produce commodity $\mathrm{CO}_{2}$ for food processing and other industries [143]. Over the past two decades, however, applications of CCS to fossil fuel power plants have been the primary focus of R\&D programs and demonstration projects worldwide, resulting in additional experience in that sector. Today, with an increasing focus on $\mathrm{CO}_{2}$ capture for a broader array of industrial applications, it is important to reflect on experiences from the power sector focus and the extent to which that is transferrable to $\mathrm{CO}_{2}$ capture from the industrial sector. 
The nine-point Technology Readiness Level (TRL) scale, first created by NASA for spacecraft applications, has become one of the most widely-used metrics of maturity of a technology in use by industry [144]. The original NASA descriptions of each level have been modified into a number of genera ${ }^{19}$ TRL definitions published by organisations such as the European Commission, Electric Power Research Institute (EPRI), DOE, and IEA (see Appendix H). Those TRL definitions have been transferred over sectors and have also been used to measure the development of $\mathrm{CO}_{2}$ capture technologies. In particular, non-sector specific TRL definitions for $\mathrm{CCS}$ or $\mathrm{CO}_{2}$ capture technologies have been published by the Zero Emission Platform (ZEP) and the EPRI [145, 146], summarized in the first two columns in Table 2-5. Generally, the TRL definitions are based on a scale 1-9, where levels 1-4 consider concept and lab scale, 5-6 consider pilot-scale systems and 7-9 consider larger demonstrations up to full commercial operation. Based on the TRL definitions set out by aforementioned institutes, several terms might lead to confusion, such as the definitions of "relevant", "system", "sub-system", and "component", or the scales, especially in the case of CCS from industry.

The direct transferability of TRLs and experience of $\mathrm{CO}_{2}$ capture technologies from one sector to another has frequently been implicitly assumed for post-combustion technologies as those are add-on systems downstream the production process. However, the definition of TRLs requires an assessment of the overall system into which a new technology is placed. Thus, the TRL of a capture technology must be defined and evaluated in the context of a specific application, with new applications having lower TRLs. For instance, while chemical absorption with MEA and proprietary solvents have been tested at commercial scale, emerging configurations and/or solvents are -by definition- of lower maturity. The same holds for oxyfuel and pre-combustion capture technologies, as these may require further modifications and integration specific to the industrial process considered.

Essentially, therefore, the transferability of experience or TRL may be limited to the particular $\mathrm{CO}_{2}$ capture system and application (as described in the TRL definition as "relevant conditions" in Table 2-5). In addition, the concept of System Readiness Level (SRL) in advanced fossil energy applications [147] has been introduced to emphasize that the maturity of new systems depends not only on the stand-alone technology but also its maturity in the specific environment. In the context of $\mathrm{CO}_{2}$ capture, the concept of SRL allows to reflect that the maturity of a specific capture system is linked to the level of technology development in conditions representative of the targeted industrial application.

While TRLs of different $\mathrm{CO}_{2}$ capture technologies for the power application have already been assessed [148], these TRLs cannot be directly used on different industrial applications due to the wide range of production processes and their associated characteristics, and the integration of the $\mathrm{CO}_{2}$ capture system with the industrial core process. Once again, an example of interest is the benchmark solvent, MEA-based chemical absorption process, and improved conventional solvents. These are and will be used, and thus demonstrated, at large-scale for several types of industrial applications in the coming few years. This is, for example, the case in the Abu Dhabi Al Reyadah/Emirates Steel CCS and Longship projects, in the steel and cement industries respectively, amongst others at FEED study stage (BEIS, 2018). In addition, the calcium looping and sorption-enhanced water-gas shift (SEWGS) processes, which are at TRL 4-6 in the power sector, are expected to make significant progress on the TRL scale for industrial applications over the next few years. Specifically, calcium looping is planned to reach TRL 7 for cement production through the CLEANKER project [149], while SEWGS should reach TRL 7 for iron and steel manufacturing through the STEPWISE project by 2021 and 2020 respectively [150]. Experience on the CALIX $\mathrm{CO}_{2}$ separation technology has been transferred from the magnesium production industry (TRL 9) to the cement production and shall reach TRL 7 through the LEILAC project [151].

\footnotetext{
19 The term general here is meant as non-CCS specific.
} 
Table 2-5. Relevant TRL definitions of $\mathrm{CO}_{2}$ capture systems in existing literature.

\begin{tabular}{|c|c|c|c|c|c|c|c|}
\hline \multirow[b]{2}{*}{ TRL } & \multirow{2}{*}{$\begin{array}{l}\text { Generic, non-sector } \\
\text { specific definition } \\
\text { European } \\
\text { Commission, } \\
\text { Horizon2020 }\end{array}$} & \multicolumn{4}{|c|}{ Non-sector specific definitions applied to CCS } & \multicolumn{2}{|c|}{ Definition applied to CCS in industrial sectors } \\
\hline & & $\begin{array}{l}\text { Zero emissions platform [145] } \\
\text { TRL definition based on NETL and } \\
\mathrm{H} 2020 \text { definition but adapted to expand } \\
\mathrm{CO}_{2} \text { capture and storage. }\end{array}$ & & $\begin{array}{l}\text { Electric Power Research Institute } \\
\text { [142] }\end{array}$ & & $\begin{array}{l}\text { Industrial process with } \mathrm{CO}_{2} \text { capture } \\
\text { based on } \mathrm{H} 2020 \text { definition but adapted to } \mathrm{CCS} \text { in } \\
\text { industrial sectors }\end{array}$ & \\
\hline 9 & $\begin{array}{l}\text { Basic principles } \\
\text { observed }\end{array}$ & $\begin{array}{l}\text { Actual system proven in operational } \\
\text { environment (competitive manufacturing } \\
\text { of full system, at scales of several } 100 \mathrm{~s} \\
\text { of } \mathrm{MWth} \text { or around } 1 \mathrm{MtCO} / \mathrm{y} \text { stored) }\end{array}$ & $\begin{array}{l}\text { Full } \\
\text { Commercial } \\
\text { Application }\end{array}$ & Normal commercial service & Demonstration & $\begin{array}{l}\text { Actual system proven in operational process } \\
\text { (with product quality maintained), and } \\
\text { competitive manufacturing of full system. } \\
\text { Technology is commercially available for the } \\
\text { specific industry. }\end{array}$ & $\begin{array}{l}\text { Full commercial } \\
\text { application }\end{array}$ \\
\hline 8 & $\begin{array}{l}\text { Technology concept } \\
\text { formulated }\end{array}$ & $\begin{array}{l}\text { System complete and demonstrated at } \\
\text { industrial scales of } 10 \mathrm{~s} \text { of } \mathrm{MW}_{\text {th }} \text { or } 0.1 \text { to } \\
1 \mathrm{MtCO}_{2} / \mathrm{y} \text { stored }\end{array}$ & Demonstration & $\begin{array}{l}\text { Commercial demonstration, full } \\
\text { scale deployment in final form }\end{array}$ & & $\begin{array}{l}\text { System complete and qualified in operational } \\
\text { process (with product quality maintained). First } \\
\text { of a kind commercial system is installed and } \\
\text { works. }\end{array}$ & Demonstration \\
\hline 7 & $\begin{array}{l}\text { Experimental proof of } \\
\text { concept }\end{array}$ & $\begin{array}{l}\text { System prototype demonstrated in } \\
\text { operational environment (industrial pilots } \\
\text { operating at } 10 \text { s of } \mathrm{MWth} \text { and/or } \\
\text { separating } 10 \mathrm{~s} \text { of } \mathrm{kt} \mathrm{CO}_{2} / \mathrm{y} \text { ) }\end{array}$ & Pilot & $\begin{array}{l}\text { Sub-scale demonstration, fully } \\
\text { functional prototype }\end{array}$ & & $\begin{array}{l}\text { System prototype demonstration in operational } \\
\text { environment (with product quality maintained). } \\
\text { For end-of-pipe technologies this means a full } \\
\text { prototype operated with flue gas from } \\
\text { operational process. } \\
\text { For technologies that are highly integrated with } \\
\text { the core process, this means a prototype where } \\
\text { all critical sub-systems are fully integrated. }\end{array}$ & Pilot \\
\hline 6 & $\begin{array}{l}\text { Technology validated } \\
\text { in the lab }\end{array}$ & $\begin{array}{l}\text { Technology demonstrated in relevant } \\
\text { environment (steady states at industrially } \\
\text { relevant environments: pilots in the } \mathrm{MWth} \\
\text { range and/or separating } 1 \text { to } 10 \mathrm{kt} \mathrm{CO}_{2} / \mathrm{y} \text { ) }\end{array}$ & & $\begin{array}{l}\text { Fully integrated pilot tested in a } \\
\text { relevant environment }\end{array}$ & Development & $\begin{array}{l}\text { Technology demonstrated in environment } \\
\text { relevant to operation in the specific industry } \\
\text { process (conditions replicating industrial } \\
\text { operation) with product quality maintained. } \\
\text { Trace elements should be included in flue gas if } \\
\text { relevant. } \\
\text { Demonstration of the sub-systems affected by } \\
\text { the product conditions may be sufficient if the } \\
\text { full system is demonstrated at TRL } 6 \text { or higher } \\
\text { for other applications (e.g. power plants). }\end{array}$ & \\
\hline 5 & $\begin{array}{l}\text { Technology validated } \\
\text { in relevant } \\
\text { environment }\end{array}$ & $\begin{array}{l}\text { Technology validated in relevant } \\
\text { environment (pilots operated at } \\
\text { industrially relevant conditions at } 0.05-1 \\
\text { MWth) and/or less than } 1 \mathrm{ktCO} / \mathrm{y} \\
\text { captured/stored }\end{array}$ & Small Pilot & $\begin{array}{l}\text { Sub-system validation in a relevant } \\
\text { environment }\end{array}$ & & $\begin{array}{l}\text { Technology validated in environment relevant to } \\
\text { operation in the specific industry process } \\
\text { (conditions replicating industrial operation). } \\
\text { Trace elements should be included in flue gas if } \\
\text { relevant. } \\
\text { Validation of critical sub-systems is sufficient. }\end{array}$ & Small pilot \\
\hline 4 & $\begin{array}{l}\text { Technology } \\
\text { demonstrated in } \\
\text { relevant environment }\end{array}$ & $\begin{array}{l}\text { Technology validated in the lab } \\
\text { (continuous operated pilots at lab scale } \\
<50 \mathrm{~kW} \text { th) }\end{array}$ & Lab/Bench & $\begin{array}{l}\text { System validation in a laboratory } \\
\text { environment }\end{array}$ & & $\begin{array}{l}\text { Technology validated in lab (continuously } \\
\text { operated). }\end{array}$ & Lab/bench \\
\hline 3 & $\begin{array}{l}\text { System prototype } \\
\text { demonstration in } \\
\text { operational } \\
\text { environment }\end{array}$ & $\begin{array}{l}\text { Experimental proof of concept (pilot } \\
\text { testing of key components at small bench } \\
\text { scale) }\end{array}$ & & $\begin{array}{l}\text { Proof-of-concept tests, component } \\
\text { level }\end{array}$ & Research & Experimental proof of concept. & \\
\hline 2 & $\begin{array}{l}\text { System complete and } \\
\text { qualified }\end{array}$ & $\begin{array}{l}\text { Technology concept formulated (basic } \\
\text { process design) }\end{array}$ & Concept & Formulation of the application & & Technology concept formulated. & Concept \\
\hline 1 & $\begin{array}{l}\text { Actual system proven } \\
\text { in operational } \\
\text { environment }\end{array}$ & Basic principles observed & & $\begin{array}{l}\text { Basic principles observed, initial } \\
\text { concept }\end{array}$ & & Basic principles observed. & \\
\hline
\end{tabular}

a Based on background work for technology evaluation carried out in CEMCAP [80, 152]. 
For guidance on setting TRLs, definitions for CCS in industrial sectors are included in the last two columns in Table 2-5. These definitions include requirements with regards to impact of CCS implementation on product quality, plant maintenance (including start-up/shut-down and unforeseen disturbances to the industrial plant operation), and operation of the CCS system under the specific conditions of the facility (e.g. flue gas composition, temperature, pressure, and considering integration). While little attention has been paid to these aspects in past cost evaluations of CCS from industry, technology maturity is an important element to consider in selecting contingencies for investment assessments (see Chapter 1). Similarly, technology maturity will also impact uncertainties in technical and cost performances [5]. Finally, further work is needed to link contingency cost assumptions to TRL values more explicitly.

\subsection{Conclusions}

With the increasing interest in $\mathrm{CCS}$ to support $\mathrm{CO}_{2}$ emission reductions from industry, a better understanding of costs is required to support decision-makers and guide research to improve the performance and reduce the cost of promising new options. While extensive studies have investigated the techno-economic performance of CCS applied to industrial sources, wide differences in cost estimates have been observed. While this is due in part to differences in the cases studied and the choice of capture technology, a significant part arises from aspects related to cost assessment methods and assumptions (cost metric definitions, energy costs, retrofitting cost, system boundaries, and other factors). Building on a previous CCS costing guideline papers [6], the present work aimed to contribute to the development of improved guidelines for cost evaluation of CCS from industrial applications. In particular, the following key messages can be extracted:

- Several publicly available, transparent, and detailed techno-economic studies exist for different industrial sectors (e.g. iron and steel, cement, refinery, hydrogen, ammonia/urea and methanol, pulp and board). These studies provide a high level of technical and cost details on the industrial facilities considered, which can be used to strengthen future evaluation of CCS from such facilities. Furthermore, these studies have also performed detailed evaluations of currently available $\mathrm{CO}_{2}$ capture technologies which can be used as a base case in comparative assessments involving new technologies. However, it is worth noting that most of these detailed studies are based on European locations and that some industry sectors are not yet studied in sufficient detail (e.g. waste-to-energy, offshore oil and gas production facilities, petrochemicals...).

- The same basic cost metrics used for CCS from power plant are relevant to industrial processes, although in some cases these may be calculated differently. Furthermore, a key challenge that might arise in the calculation of cost metrics for industrial plants is that many processes result in multiple products. In such cases, the cost of CCS may need to be allocated across these products when reporting costs on a normalized basis (e.g., cost per unit of product). While different allocation bases (flow, energy, market value) exist to distribute these (or other) costs, there is no standardized methodology currently in use. When possible, it is thus recommended to report CCS costs using more than one allocation method as this will provide insights into the impact of different methods on cost performance.

- The origin and production/supply strategy of the steam and electricity required for the $\mathrm{CO}_{2}$ capture process may vary considerably on a case-by-case basis and have a significant impact on its cost and associated $\mathrm{CO}_{2}$ emissions, and thus on the $\mathrm{CO}_{2}$ avoidance cost. It is recommended that transparent scenarios of realistic (future) heat and power supply strategies are included in cost evaluations and that considered methods and assumptions are explicitly reported. The supply strategy, the cost and associated emissions intensity of a heat and power supply can also be site- 
specific and dependent on external parameters such as energy prices, which can change significantly over time. To deal with these uncertainties, analysts are encouraged to develop scenarios for plausible combinations of future energy and carbon prices, so as to clearly understand the impact of possible outcomes.

- Few studies properly account for the cost of retrofitting $\mathrm{CO}_{2}$ capture from existing facilities. As illustrated in the literature, these costs can vary considerably on a case-by-case basis and should thus be properly accounted in studies assessing retrofit applications of CCS. Particular attention should be paid to the following aspects: economic impact of potentially required plant production stoppages, impacts on the main output product quality and plant operation, flue gas treatment requirements, spatial constraints in plant sites, flue gas interconnection and utilities connection costs.

- Costs associated with $\mathrm{CO}_{2}$ transport and storage are often assumed to be a fixed unit cost per tonne of $\mathrm{CO}_{2}$, independent of the expected transport and storage conditions (distance, volume, type of transport and storage). While there is significant room to improve the quality of transport and storage cost estimates, it is recommended that any such estimates be based on at least the applicable $\mathrm{CO}_{2}$ flowrate, type of transport, transport distance and type of storage. Illustrative literature values are provided to support such preliminary estimates when detailed evaluations are not possible. It is worth noting that these considerations hold for both CCS from industry and power.

- Technology maturity is an important factor in cost estimates, usually accounted for through the inclusion of process contingency costs for different levels of maturity and experience. Caution must be taken, however, when considering transferability of technology maturity from power sector applications to various industrial sectors. Impacts to consider include potential effects of CCS on product quality, plant maintenance, and operation of the CCS system under the specific conditions of the industrial facility.

Finally, the authors strongly recommend that future studies of industrial CCS applications make efforts to better document the adopted costing methodology, assumptions, and data sources, and to incorporate sensitivity analyses and scenarios for key assumptions to increase the usefulness and robustness of cost estimates. 


\section{Chapter 3: Toward improved guidelines for uncertainty analysis of carbon capture and storage techno-economic studies ${ }^{20}$}

\subsection{Introduction}

Sound uncertainty analysis is critical to the informed interpretation of carbon capture and storage (CCS) techno-economic analyses (TEA) $[8,153]$. It can provide valuable insight into the impacts of assumptions and underlying model structure, and give an indication of model quality and robustness, as well as of how reliable the outputs of modelling studies are or can be. Without uncertainty analysis, the actual meaning and importance of CCS cost results from any techno-economic study are difficult to judge, especially for the target audience of such studies, who are often not involved in their production.

Over the years, many publications on uncertainty evaluation methods and approaches have found their way to the scientific domain (e.g., [154-156]), as have publications on the application of uncertainty analysis to TEA and CCS costing specifically (e.g., [157-159]). However, it is notable that often when uncertainty analysis methods are applied in TEAs, mostly simplified methods tend to be used (e.g., single parameter sensitivity analysis) whereas other (more complex) methods could provide additional valuable insight. Furthermore, new and promising uncertainty methods appear to remain unknown to (or unapplied by) the CCS TEA community. We believe this is partly due to unawareness of the full space of uncertainty analysis options, as well as a lack of guidance on when and how to use such options. Therefore, a critical review of the different options, complemented with guidelines on uncertainty evaluation can i) raise awareness to this issue, and ii) increase the effective and fit-forpurpose use of uncertainty analysis in CCS techno-economic studies, hopefully leading to improved understanding and communication of their results.

Building on a previous CCS costing guideline paper [6], this work sets out to provide a review of, and guidelines on, uncertainty analysis methods for use in CCS TEA. It combines knowledge and experience acquired in academia, research institutes, and non-governmental organizations (NGOs). The overarching goal of this chapter is to advance the sound and fit-for-purpose use of uncertainty analysis in CCS TEA by providing practitioners and users with a reference document of relevant methods, tools, and approaches, and a guideline of when and how to use them. These guidelines do not intend to provide an exhaustive account of every available method, rather to showcase different methods over a broad spectrum that can act as an illustration of the type of methods available. Naturally, these guidelines are equally applicable to TEAs of $\mathrm{CO}_{2}$ utilisation and negative emission technologies.

\footnotetext{
${ }^{20}$ This chapter was authored by Mijndert van der Spek, Timothy Fout, Monica Garcia, Vishalini Nair Kuncheekanna, Michael Matuszewski, Sean McCoy, Joshua Morgan, Shareq Mohd Nazir, Andrea Ramirez, Simon Roussanaly, and Edward S. Rubin. It corresponds to a post-print version of the paper "Uncertainty analysis in the techno-economic assessment of $\mathrm{CO}_{2}$ capture and storage technologies." Critical review and guidelines for use. International Journal of Greenhouse Gas Control 100, 103113. 2020" published under a Creative Commons license CC BY and is available in open access at https://doi.org/10.1016/j.ijggc.2020.103113. The corresponding author for this chapter is Mijndert van der Spek (e-mail address: $m$.van derspek@,hw.ac.uk).
} 


\subsection{Scope and background}

\subsubsection{Audience for this chapter}

These guidelines target two types of audiences: techno-economic analysis practitioners and the users of techno-economic studies (Table 3-1). The first group is composed of people involved in the development, modelling, costing, and analysis of (new) CCS technologies. They will be found mainly in research and development (R\&D) agencies, academia, and in industrial organisations.

The second group are the users of TEA studies, as earlier described in Rubin et al., [8]. They are mostly technology (R\&D) and policy decision-makers. The purpose for this group is twofold: first to help them gain an understanding of the role of uncertainty and how it may affect assessments of technology performance, and second to provide an overview of available uncertainty analysis methods and their use, so that decision-makers can request TEA practitioners to undertake the specific analysis that may fit their information needs best. A key example of the second group is funding agencies, who on a regular basis need to make informed decisions on funding technology proposals.

Table 3-1. Target audiences for this guideline document

\begin{tabular}{|c|c|c|c|}
\hline Audience & Government & Industry & NGO's \& universities \\
\hline $\begin{array}{l}\text { Practitioners: process } \\
\text { developers/modellers, } \\
\text { cost engineers and } \\
\text { technology analysts in: }\end{array}$ & - R\&D agencies & $\begin{array}{l}\text { - Operators } \\
\text { - Vendors } \\
\text { - A\&E firms } \\
\text { - Venture capital } \\
\text { - R\&D organisations }\end{array}$ & - Academia \\
\hline $\begin{array}{l}\text { Users of CCS techno- } \\
\text { economic studies in: }\end{array}$ & $\begin{array}{l}\text { - Policymakers } \\
\text { - Analysts } \\
\text { - Regulators } \\
\text { - R\&D agencies } \\
\text { - Funding agencies }\end{array}$ & $\begin{array}{l}\text { - Operators } \\
\text { - Vendors } \\
\text { - A\&E firms } \\
\text { - Venture capital } \\
\text { - R\&D organisations }\end{array}$ & $\begin{array}{l}\text { - Environmental } \\
\text { - Media } \\
\text { - Academia } \\
\text { - Foundations }\end{array}$ \\
\hline
\end{tabular}

\subsubsection{Types of techno-economic analyses and candidate parameter categories for uncertainty analysis}

Before venturing into available uncertainty analysis methods, it is worthwhile to provide a rough description of the type of techno-economic models found in today's CCS literature, since different types of studies may require different types of uncertainty analysis. Table 3-2 presents a simplified overview of the types of techno-economic models and parameter categories that can be candidates for uncertainty evaluation (based on an earlier publication [160]). On the one end, there are simplified techno-economic models that, for instance, are used to get a first rough idea of technical and economic feasibility. These models may be based on simple first principles or more black box technology descriptions, and their economics are often derived from future projections of known equipment cost. On the other end of the spectrum are detailed techno-economic studies based on full physical (rigorous) technology models and detailed "bottom-up" cost models, such as the engineeringeconomic models often used in studies by e.g., the US Department of Energy's National Energy Technology Laboratory (DOE/NETL), the International Energy Agency Greenhouse Gas Programme (IEAGHG), and the Electric Power Research Institute (EPRI). In between, there are more or less detailed technical and economic modelling studies, here called intermediate complexity models, often using, e.g., shortcut models for technology description, and/or partial process design and equipment 
lists for costing. The choice for a certain model type may depend on the goal of the study, the availability of data and physico-chemical models, and the technology readiness level (TRL).

Table 3-2 is not meant as an exhaustive list of all parameters that need to be scrutinised, but rather provides an illustration of complexity levels. As the table clearly shows, the more detailed and complex the TEA, the more input parameters and models used, and therefore the more parameters that can, or should, be included in the uncertainty analysis. Also, especially for rigorous process models, it is good practice to investigate the effect of model structure. This, for example, could be reflected in the choice of models for mass transfer, as it may have a large effect on the technical process, and therefore its performance and cost (see e.g. [161, 162] for a discussion on how the choice of mass transfer models is relevant in solvent-based $\mathrm{CO}_{2}$ capture systems, but it equally applies to other types of capture technology).

Table 3-2. Indicative technical and economic model types and candidate parameter (categories) for uncertainty scrutinization, based on [160].

\begin{tabular}{|c|c|c|c|c|}
\hline & \multicolumn{2}{|c|}{ Technical models } & \multicolumn{2}{|c|}{$\begin{array}{l}\text { Economic models } \\
\end{array}$} \\
\hline $\begin{array}{c}\text { Model } \\
\text { complexity } \\
\text { level }\end{array}$ & Description & $\begin{array}{c}\text { Potential parameter } \\
\text { categories to } \\
\text { scrutinise }\end{array}$ & Description & $\begin{array}{c}\text { Potential parameter } \\
\text { categories to } \\
\text { scrutinise }\end{array}$ \\
\hline Rigorous & $\begin{array}{l}\text { Typically, full } \\
\text { physical models based } \\
\text { on first principles, } \\
\text { including detailed } \\
\text { flowsheets, mass and } \\
\text { heat transfer, detailed } \\
\text { kinetics, recycles, } \\
\text { etcetera. }\end{array}$ & $\begin{array}{l}\text { Below parameter } \\
\text { categories as well as } \\
\text { mass \& heat transfer } \\
\text { models, chemical } \\
\text { kinetics models }\end{array}$ & $\begin{array}{l}\text { Typically, detailed } \\
\text { economic estimates, } \\
\text { based on a detailed } \\
\text { equipment list, using } \\
\text { individual escalation } \\
\text { and/or scaling factors } \\
\text { and including all } \\
\text { capital and } \\
\text { operational costs }\end{array}$ & $\begin{array}{l}\text { All of the below plus } \\
\text { individual escalation } \\
\text { and/or scaling factors, } \\
\text { detailed capital and } \\
\text { operational cost } \\
\text { factors }\end{array}$ \\
\hline Intermediate & $\begin{array}{l}\text { E.g., short-cut models, } \\
\text { excluding part of the } \\
\text { physical description } \\
\text { (often mass transfer, } \\
\text { heat transfer, chemical } \\
\text { kinetics) }\end{array}$ & $\begin{array}{l}\text { Below parameter } \\
\text { categories as well as, } \\
\text { amongst others, } \\
\text { chemical equilibrium } \\
\text { models and state } \\
\text { parameters }(\mathrm{P}, \mathrm{T})\end{array}$ & $\begin{array}{l}\text { E.g., combinations of } \\
\text { bottom up and top- } \\
\text { down methods, using } \\
\text { partial equipment lists } \\
\text { and Lang/Hand type } \\
\text { escalation factors }\end{array}$ & $\begin{array}{l}\text { All of the below plus } \\
\text { equipment sizes, } \\
\text { purchased equipment } \\
\text { costs, escalation } \\
\text { factors }\end{array}$ \\
\hline Simplified & $\begin{array}{l}\text { Often mass and } \\
\text { energy balance } \\
\text { models based on } \\
\text { literature or } \\
\text { experimental results }\end{array}$ & $\begin{array}{l}\text { Mass and energy } \\
\text { input and output flows }\end{array}$ & $\begin{array}{l}\text { Typically, top-down } \\
\text { cost estimates, e.g., } \\
\text { exponent models, } \\
\text { using cost estimates } \\
\text { from earlier studies }\end{array}$ & $\begin{array}{l}\text { Financial parameters } \\
\text { (lifetime, discount } \\
\text { rate, etcetera); fuel \& } \\
\text { consumables cost; } \\
\text { scaling exponents }\end{array}$ \\
\hline
\end{tabular}

\subsubsection{What do the existing TEA guidelines say about uncertainty assessment?}

As discussed in the introduction, a comprehensive set of guidelines for uncertainty analysis of technoeconomic studies of CCS technologies is currently lacking. Two widely used guideline documents for CCS TEA in general, The DOE/NETL Quality Guidelines for Energy System Studies (QGESS [163]) and the European Best Practice Guidelines for Assessment of $\mathrm{CO}_{2}$ Capture Technologies [164], provide a note on uncertainty analysis. Both recommend the use of sensitivity analysis to help generate an understanding of uncertainty in input data, financial assumptions, and state of technology development. They, however, limit themselves to the most simple of sensitivity analyses (one-at-atime sensitivity analysis, see 3.3.3.1), without discussing alternative methods and without providing guidance on how to undertake uncertainty analysis in a methodologically sound way. 
The techno-economic studies by organisations such as the IEAGHG or the Zero Emissions Platform (ZEP), are also often used as guidelines for good TEA practice. The IEAGHG studies generally use the standardised IEAGHG techno-economic and financial parameters for their studies (e.g., [37]). The IEAGHG studies generally include sensitivity analyses, aiming to offer a picture of how changes to the standard IEAGHG assumptions could impact CCS costs. The key parameters generally investigated are fuel prices, discount rate, discount rate after plant closure, plant lifetime and $\mathrm{CO}_{2}$ transport and storage costs, subject to the objective of the study [84, 86, 165]. Recent studies [88] also included assessments of potential techno-economic scenarios, where the authors a) explored technical parameters that can have a significant impact on the $\mathrm{CO}_{2}$ capture costs; $b$ ) provided overviews of the TRLs of the $\mathrm{CO}_{2}$ capture technologies and their impact on costs, c) highlighted technical differences in the literature which make cost-reviews more challenging and homogenised costs under one unique framework, and d) provided an analysis on the energy assumptions and integration of the $\mathrm{CO}_{2}$ capture system with the original facility, and its impact on costs. The TEA studies by ZEP only investigate sensitivity to plant efficiency and capital costs [166] for $\mathrm{CO}_{2}$ capture plants and extends that with operational costs, distance, and utilisation level for $\mathrm{CO}_{2}$ transport [120]. Only in their $\mathrm{CO}_{2}$ storage costing study, ZEP also included sensitivities on high-level technical parameters like field and well capacity [121].

The abovementioned guideline documents and techno-economic studies use, what are often called, local sensitivity analysis methods for uncertainty analysis, especially the one-at-a-time, and one-way type of sensitivity analysis (see section 3.3.3.1). Another observation is that these studies mostly focus their sensitivity analyses on economic input parameters (although there are exceptions). When addressing technical parameters, they do so mostly at high level, aggregated, parameters such as power plant efficiency, rather than the underlying technical input parameters that might exist in a firstprinciple process model.

\subsection{Uncertainty analysis}

\subsubsection{Definitions of uncertainty analysis}

In the CCS TEA literature, Rubin et al. [8], make a distinction between uncertainty, variability and bias. Therein, the author defined uncertainty to reflect "a lack of knowledge about the precise value of one or more parameters affecting CCS costs" ([8]:187). In this definition, uncertainty exists solely in the value of parameters. Variability was defined to refer "to the different value a given parameter may take on (for example, across a collection of facilities, or at different points in time at a given facility). In this case, the values of the parameters are assumed to be known (or knowable), and thus subject to quantitative data analysis" ([8]:187). This means that the variability of a given parameter can be measured and can thus be quantified by, e.g., a probability density function (PDF). This would be much more difficult (or arbitrary) in the case of uncertainty, where the lack of knowledge (true uncertainty) would hamper defining a precise $\mathrm{PDF}^{21}$. Furthermore, the author defined bias to refer "to assumptions that skew an analysis in a particular direction while ignoring other valid alternatives,

\footnotetext{
21 This differentiation between uncertainty and variability relates closely to what is called epistemic and aleatoric uncertainty in the uncertainty quantification (UQ) literature [167]. The UQ community thus refers to both as uncertainty, but of a different nature. Epistemic uncertainty is also called reducible uncertainty, ignorance uncertainty or subjective uncertainty [168]. Aleatoric uncertainty, on the other hand, refers to inherent variability of a quantity of interest or unpredictability due to stochasticity [167], indeed equivalent to Rubin's definition of variability [8].
} 
factors or data" ([8]:188), meaning that the outcomes of a study may change when favouring one input parameter value over another.

Other scholars use a wider definition of uncertainty by focusing on all kinds of knowledge or information, rather than only on parameters. For instance, "incomplete information about a particular subject" ([169]:387), "lack of confidence in knowledge related to a specific question" ([170]: 504), and "any deviation from the unachievable ideal of completely deterministic knowledge of the relevant system" ([171]:5). These three definitions all focus on the lack of knowable knowledge, values, or information, and in that way, they are closest to Rubin's definition of uncertainty.

We here adopt a wide definition of uncertainty, that includes both parameter [8, 160, 172] and nonparameter uncertainty (i.e., knowledge uncertainty [173] and uncertainty in the model structure and boundaries: methodological, model structure, and contextual uncertainty $[160,172])$. At the same time, we embrace the difference between uncertainty and variability (or epistemic and aleatoric uncertainty), because they play an important role in the selection of uncertainty analysis methods, as we will discuss later.

\subsubsection{Purposes of uncertainty analysis}

Before outlining different uncertainty analysis methods that are commonly used in CCS technoeconomic analysis, we first discuss the different purposes uncertainty analysis may have. The first, straightforward, purpose is to provide insight into potentially different outputs as a result of different input assumptions. This is a way to answer "what if", or, diagnostic, type of questions [3, 174]. A more sophisticated purpose can be to provide an estimate of a certain output happening. This relates more to "what will", or prognostic questions [3, 174], because the model specifies a certain outcome of happening with a certain probability. Related to this, uncertainty analysis can help understand which input parameters influence the model outputs most and should therefore be scrutinised and/or parametrised most thoroughly (for instance, through Factor Prioritisation, FP, [174]). This can help answer the question of "where to put most effort" when quantifying model input values. Conversely, uncertainty analysis may provide insight into the parts of the model(s) that have less influence on the outputs, thereby answering "which parts of the model can we simplify" and providing a basis for model reduction (Factor Fixing, FF [174]). An advanced use of uncertainty analysis is model testing, i.e., testing how a model behaves when fed with extreme parameter values or scenarios. If the model behaves as expected (i.e., provides the expected outputs), it increases confidence that the model structure is correct. If unexpected outputs occur, this may indicate that model equations are incorrect, or incorrectly implemented. Finally, uncertainty analysis can also generate insight into the strength of models and/or the input data fed to them (further discussed in 3.3.4.1), which is of great importance when models are used for policy and decision making.

\subsubsection{Established uncertainty analysis methods}

This section describes established uncertainty analysis methods, i.e., methods that have wellestablished principles and that have been commonly used in techno-economic analysis for decades. We start out with a general description of sensitivity analysis and local sensitivity analysis methods and then continue with a description of global (e.g., Monte Carlo based) uncertainty analysis. This section ends with describing pitfalls and good practices for the established methods.

To allow explanation of the different methods, a very simple and generic mathematical representation of uncertainty analysis is introduced in the main text:

$$
\mathbf{y}=g(\mathbf{x})
$$


Where, $g$ is a techno-economic model (i.e., a mathematical function). Despite the simple representation here, $g$ can be a very complex system of equations solved analytically or numerically. $\mathbf{x}$ is an array of $n$ model inputs $\left\{\mathrm{x}_{1}, \mathrm{x}_{2}, \ldots, \mathrm{x}_{\mathrm{n}}\right\}$ and $\mathbf{y}$ is an array of m model outputs $\left\{\mathrm{y}_{1}, \mathrm{y}_{2}, \ldots, \mathrm{y}_{\mathrm{n}}\right\}$. We call $k$ the number of parameters out of the set $n(k \leq n)$ that are actually varied in a sensitivity analysis. This will suffice the explanation of the methods below. Slightly more comprehensive mathematical representations can be found in Appendix I.

\subsubsection{Sensitivity analysis}

Sensitivity analysis (SA) studies how uncertainty in the output of a model is apportioned to different sources of uncertainty in the input of a model $[175,176]$. Its main purpose is thereby to identify which inputs most significantly impact the model, helping to prioritise the effort of a modeller on further quantifying the model inputs, while making the model more robust. The model inputs to which the output is most sensitive deserve the most rigorous quantification; the inputs to which the model is least, or not sensitive, can be allowed less stringent quantification, or can be set to fixed (sometimes even random) value. Below we describe commonly used sensitivity analysis methods used in CCS costing, distinguishing between local sensitivity analysis and global sensitivity analysis. The descriptions are made around three main aspects: definition (what), method (how), and their applicability (when).

\subsection{Local sensitivity analysis}

By far the most commonly used sensitivity analysis methods are local, meaning that one or more variables are varied around selected base, or nominal, values. This also implies that local methods do not include the uncertainties in the whole solutions space (set of potential outputs) of the model) [28]. The reason for the wide use of local methods is their ease of use and of interpretation of their results. What: Local sensitivity analysis can roughly be divided in three types: one-at-a-time sensitivity analysis, one-way sensitivity analysis, and n-ways sensitivity analysis (scenario analysis, sometimes called N-at-the-time -NAT- sensitivity analysis was excluded from below description, because it is a very special class of uncertainty analysis). The simplest local method is one-at-a-time (OAT) sensitivity analysis $[154,155]$, where the model output is only evaluated against a minimum and a maximum value of a given input parameter. This is most commonly known as the plus/minus $10 \%$ way of varying an input parameter (example in Figure 3-1). An extension to OAT sensitivity is oneway sensitivity analysis [155]. Here, one parameter at a time is varied but over its entire predetermined range, sampling multiple points. The added value is that a response to an input becomes visible between its extremes, allowing to also identify potential non-linearities between input and output (see, e.g., the IEA figure [177] in Figure 3-2). One-way sensitivity analysis can be further extended to multiple ways sensitivity analysis (or n-ways sensitivity analysis) [155]. This method varies multiple parameters at a time, and uses for each parameter, a set of different values from its entire range. It has the ability to investigate the model output space as function of many parameters with less runs than would be required when only using one-way-sensitivity. In addition, it is able to show interdependencies between input parameters (Example provided in Figure 3-3).

How: The main methodological step in sensitivity analyses is to choose the perturbations to the nominal value of the parameters under investigation. This is only trivial when input-output relations are linear and there is no interaction between input parameters. For OAT analysis for instance, it is common practice to vary each input parameter with a fixed percentage, e.g. $+/-10 \%$, or $+/-50 \%$. Note however, that in case of non-linear relations a variation of $10 \%, 50 \%$, or other, may lead to a different ranking of sensitivity to inputs. Alternatively, each parameter can be given a different minimum and 
maximum, for instance based on expert knowledge of the minimum and maximum values a parameter can have. From a purist perspective, the former approach (using the same plus and minus) is more appropriate, since the local nature of OAT analysis strictly does not allow a statement of the total variance of output $\mathbf{y}$ as a function of input $\mathbf{x}$. Rather, OAT analysis merely produces a ranking of sensitivity to input parameters when varied close to their nominal value. However, from a pragmatic perspective, it seems illogical to vary all parameters with the same percentage around their nominal value if it is known that their real ranges differ substantially, and the practitioner would like to get a first impression of the maximum range of the output parameters. The methodologically correct use of strict OAT analysis is limited, and there may be good reason to choose another method.

One-way sensitivity analysis in this perspective is more versatile than OAT sensitivity analysis, because it allows to sample multiple perturbations from the input parameter's nominal value. Here, it is advised to choose the minimum and maximum values more widely, and preferably according to known, or realistic, limits. The main choice here is rather how many points to include, and where to sample them. For (or when suspecting) non-linear relations, it is advisable to add more points in those sections of the curve that are likely to show the highest non-linearities. For inputs with a linear relation to output, it would suffice to only use one minimum and maximum values without sampling points in between. These considerations also apply to n-way sensitivity analysis.

An important consideration for the choice of method is its computational cost (i.e., the amount of time that it takes to run an analysis). It also depends on the computing hardware available: the computational cost of running an analysis is much higher for any model on a two-core laptop than on a supercomputing cluster where multiple dozens of cores work in parallel. For OAT, the computational cost can be estimated as $C=2 k+1$ [155], where $k$ is the number of inputs that is varied. The computational cost of one-way sensitivity analysis is obviously higher than OAT sensitivity analysis: $C=(2+a) k+1$, where $a$ is the amount of sampled points in addition to its extremes and the base case. Because n-ways sensitivity analysis varies input parameters simultaneously, its computational cost is typically lower than that of one-way sensitivity analysis. As for one-way sensitivity analysis, $C$ depends on the number of sampled values of each input $x_{i}$. In addition, it is more difficult (and thus requires more computational effort) to determine the influence of individual parameters when using n-ways analysis. For example, $C=2 k$ to calculate all order sensitivities for generalised $\mathrm{min} / \mathrm{max}$ full factorial designs, i.e., when only two values (a minimum and a maximum) for each input $x_{i}$ are sampled; $C=2 k+2$ to calculate first order (individual), interaction and total order sensitivity indices for $\min / \max$ full factorial designs $[155,174]$. Similarly, when a range of values for each $x_{i}$ is sampled, the computational cost increases to $C=(2+a) k$, with $a$ being the amount of sample points in addition to its extremes.

When: Local sensitivity analysis is best used to answer diagnostic, or "what if" type of questions, where it has less use to prognostic studies due to its local nature. It can be used very well for a first and quick screening of influential variables, both in process modelling and economic analysis. In this case it is less relevant if the nature of the input parameter variance is pure uncertainty or rather variability (see above), because we are more interested in a ranking of parameters, than in the actual predicted output of the model. Though being simple, local sensitivity analysis shows some relevant disadvantages. Its main drawback is arguably the inability to estimate the influence of variable interactions (OAT and one-way sensitivity analysis) and non-linearities (OAT sensitivity analysis) [174]. Especially in complex and highly non-linear mathematical models this can lead to a false ranking of importance [154], or to the discarding of inputs as relevant based on their single variable sensitivity, while they may actually be relevant in combination with other parameters. This means 
that local sensitivity analysis is more suited to simple models (like simple factorial cost estimates) than to complex models (like full physical process models). Also, local sensitivity analysis can be inefficient if $k$ is large and only some input parameters are influential. This is, again, especially the case for computationally intensive models.

In addition to diagnostic questions, local sensitivity analysis can also be very useful to test the model structure, by running extreme cases. If for instance, in an economic model, the discount rate is set to a very high value, the levelised cost of product should also become very high. If this is not the case, the modeller knows there may be a mistake in the model formulation.

\subsection{Common pitfalls in (local) sensitivity analysis}

The following list provides some common pitfalls, to prevent mistakes in the use of sensitivity analysis for CCS techno-economic analysis and elsewhere:

- Local sensitivity methods are perceived as sufficient, where in reality they may not fulfil the purpose of the study. This, in turn, leads to spending time applying methods that may not generate the answers that are needed, or that are less suitable given a certain scope of the problem/question.

- Showing the sensitivity of (too) many model outputs to the model inputs, thereby confusing the audience of the study [174]. Often only a few output parameters are really interesting and is better practice to show only those, while being transparent on the full number of parameters studied.

- Performing piecewise sensitivity analysis where it is not justified. Piecewise sensitivity analysis examines the model in parts or compartments. Often, uncertainty is propagated through the model compartments in non-linear and interactive ways, and piecewise analysis could therefore provide false insights. Examples could include the piecewise examination of uncertainty in a process model and a cost model, where it might be more justified to investigate the uncertainty of the integrated techno-economic model.

- Using the wrong representation of results for a chosen method. For instance, representing the results of an OAT sensitivity analysis in a spider plot. This may happen if modellers are not familiar with the full range of possible approaches and good practices of graphic representation. 
Sensitivity of LCOE ( $€ / \mathrm{MWh}$, change from a base value of 102,56)

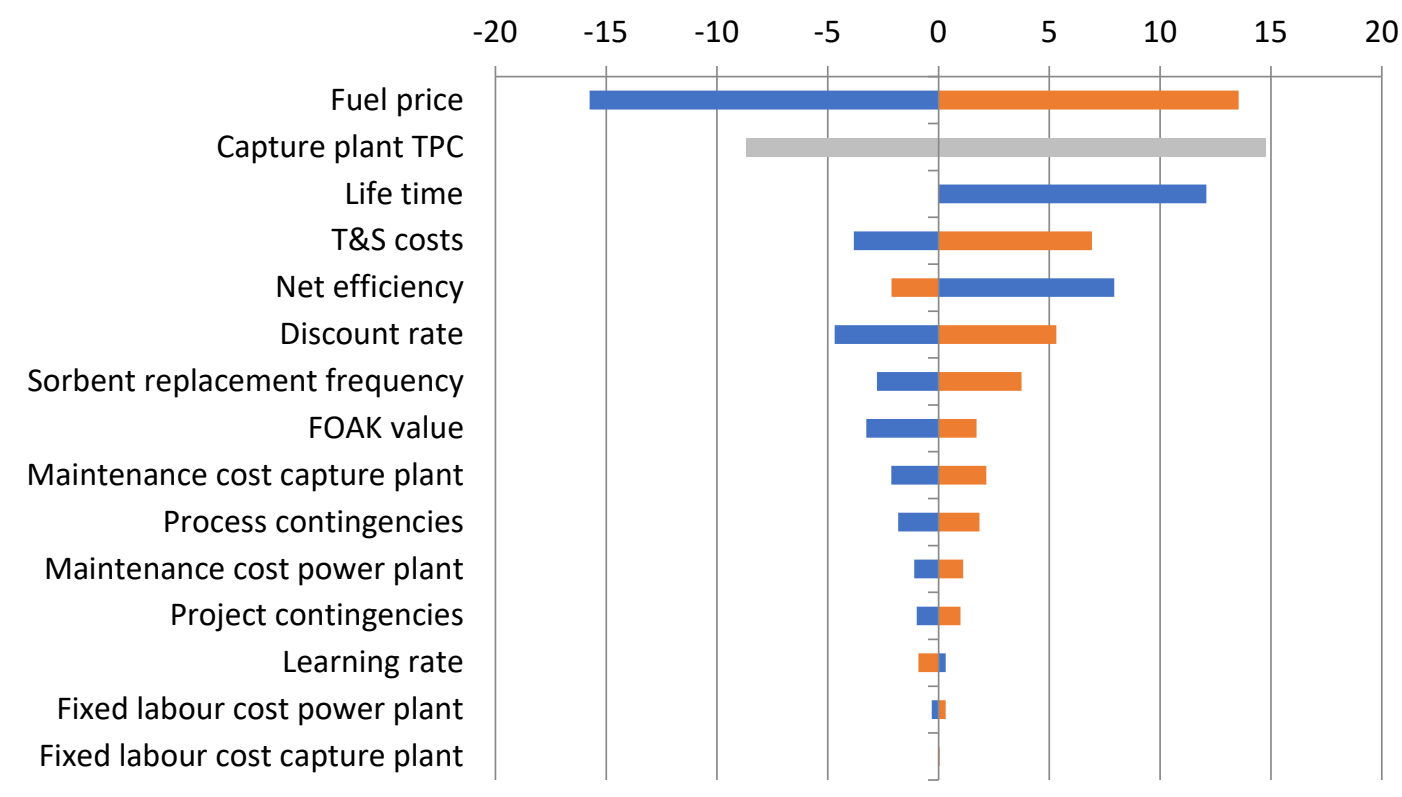

Figure 3-1. Illustrative example of OAT local sensitivity analysis: Tornado diagram representing the OAT sensitivity of the levelised cost of electricity to economic input parameters.

Figure footnote: The case study represents an NGCC plant equipped with post-combustion capture with solid sorbents using an electric swing adsorption cycle [57]. Capture plant TPC ranges (grey colour) include simultaneous variation of the engineering, procurement and construction (EPC) costs, process, and project contingencies, FOAK value, and learning rate, proving a lumped contribution of these capital cost elements.

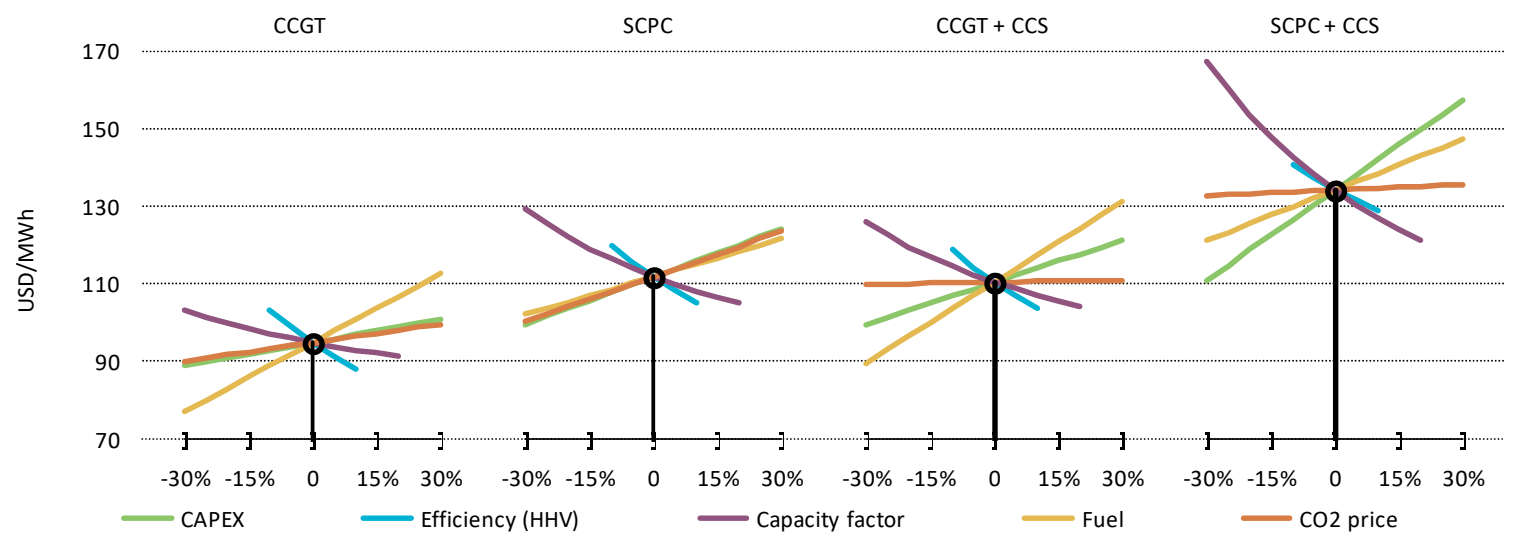

Figure 3-2. Illustrative example of one-way local sensitivity analysis showing the effect of CAPEX, efficiency, capacity factor, fuel costs and $\mathrm{CO}_{2}$ price on the LCOE of power plant with and without CCS: Figure 5.8 from the IEA Energy Technology Perspectives 2014 [177].

Figure footnote: The parameters were varied independently, leading to the typical spider webs of lines. Note the nonlinearity of some parameters, e.g., capacity factor, making one-way analysis more suitable for this parameter than OAT analysis. 


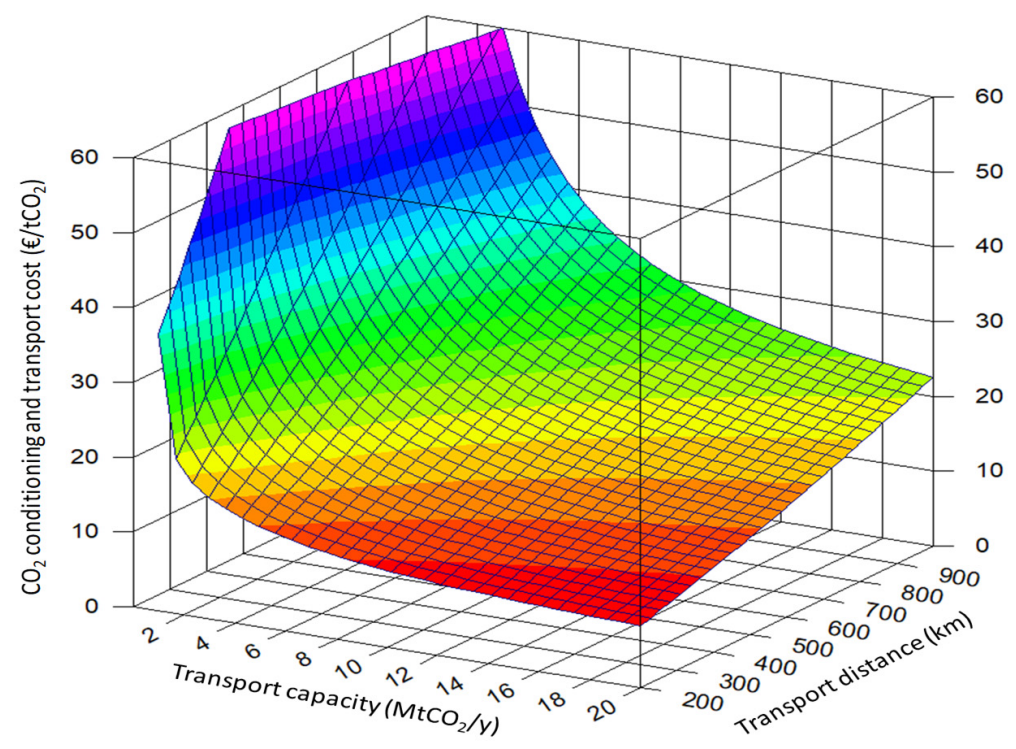

Figure 3-3. Illustrative example of $\mathrm{N}$-ways (here: 2-ways) local sensitivity analysis, based on [125], showing the impact of transport capacity and distance on the cost of $\mathrm{CO}_{2}$ conditioning and transport for an offshore pipeline infrastructure.

Figure footnote: This figure is based on the following considerations: 1) $\mathrm{CO}_{2}$ comes from an MEA-based $\mathrm{CO}_{2}$ capture unit; 2) Pipeline costs are calculated according the $\mathrm{CO}_{2}$ Europipe cost model [178] and an electricity cost of $55.5 € / \mathrm{MWh}$ is assumed; 3) Pipeline diameter is optimised for each combination of transport capacity and distance; 4) A constant utilisation rate of $85 \%$, a project duration of 25 years with an $8 \%$ discount rate are considered in the cost calculation.

\subsubsection{Probabilistic uncertainty analysis}

What: Probabilistic (global) uncertainty analysis is a step up from simple one-way or n-ways sensitivity analysis because it assigns a probability to the range of values that a parameter can have $[154,174,179]$. It not only tells something about which values the model outputs $\mathbf{y}$ may take on as a function of changes in model inputs $\mathbf{x}$, but also how likely a certain output may be (for e.g. see Figure 3-4). Global uncertainty analysis can also be used to find the global sensitivity of the outputs $\mathbf{y}$ to the input vector $\mathbf{x}$. This is different from local sensitivity in the way that the sensitivity indicators are estimated for the whole range of possible inputs and outputs (thus global), while the local sensitivity indicators only apply around the selected base value. Apart from the advantage of understanding the model input-output relationship over the whole range of parameter space, global uncertainty analysis also allows to identify the synergistic effects between model inputs.

How: Probabilistic uncertainty analysis requires assigning to each or some of the input parameters a probability density function. These functions are typically fitted on measured (but often assumed) data. Mathematically, $\mathbb{P}_{\mathbf{X}}$ are the probability distribution functions of the random vector of input parameters $\mathbf{X}=\left(X_{1}, \ldots, X_{n}\right)$ and $\mathbb{P}_{\mathbf{Y}}$ the PDFs of the random vector of output parameters $\mathbf{Y}=$ $\left(Y_{1}, \ldots, Y_{m}\right)=g(\mathbf{X})$. This results in a cumulative distribution function $F_{Y}(y)=\int f_{Y}(y)$ over the range of output realisations. Preferably, $F_{Y}(y)$ and $f_{Y}(y)$ are evaluated analytically, because of low computational cost. Practically, this is often impossible, because the mapping between $\mathbf{X}$ and $\mathbf{Y}$, denominated $g(\cdot)$, is unavailable (i.e., there is no analytical solution for $g(\mathbf{X})$ ). In such cases, the probability of $\mathbf{Y}$ can be evaluated by repeated sampling using Monte Carlo methods. 
From the PDFs of inputs and outputs, global sensitivity measures can be calculated (i.e., the measures that rank the sensitivity of model outputs to each model input), which is now also often done in CCS research. There are different methods to do this and the calculation of these measures is a separate research field (the underlying mathematics can be quite complex and sometimes not transparent). It is beyond the scope of this work to address this in detail, but there are good standard works on calculation of global sensitivity indicators in literature, e.g. [174, 175].

The characterization or quantification of input parameters with PDFs is arguably the most challenging part of global uncertainty analysis and therefore we elaborate a little further on this, without the intention to provide an exhaustive overview of PDF selection. We identify three possible approaches:

1. Hawer et al., [180] developed a guideline based on a flowchart that contains questions to be answered by the user with "yes" or "no", as shown in Appendix J. The flowchart leads the user to a recommendation for a representative uncertainty characterization method for each individual case. The guideline aimed at an audience with little to no knowledge on the terminology and or modelling in the field of uncertainty quantification.

2. In another publication [181], the principle of maximum (information) entropy was suggested. The principle of maximum entropy seeks to choose a PDF that maximizes the amount of information that a distribution can provide, subject to known constraints, as summarized in Table 3-3. This means, for instance, that when only an upper and a lower bound of a range are known, the uniform distribution is the PDF that provides the most information.

3. A third technique for obtaining a PDF is to use a 'subjective' probability distribution, that is a PDF that is based on knowledge of the process/parameter rather than data. Generally, this is accomplished by the application of formal expert elicitation following a systematic procedure. Examples of formal protocols for expert elicitation can be found in Morgan and Henrion [61], developed for the field of quantitative risk and policy analysis and the IPCC tool linking probability with linguistic descriptions of uncertainty [181].

Table 3-3: Choosing a distributions function using the principle of maximum entropy. Adapted from [181].

\begin{tabular}{|l|l|}
\hline Available information & Assigned PDF \\
\hline Upper bound, lower bound & Uniform \\
\hline Minimum, maximum, mode & Triangular \\
\hline Mean, standard deviation & $\begin{array}{l}\text { Normal or lognormal if the value physically cannot be below } \\
\text { zero }\end{array}$ \\
\hline Range, mean, standard deviation & Beta \\
\hline Mean occurrence rate & Poisson \\
\hline
\end{tabular}

When: Because of its stochastic nature, probabilistic uncertainty analysis is very capable to answer prognostic, or "what will" type of questions, as well as provide the audience with an answer on how likely such an outcome could be (see also Figure 3-4 ). It is therefore helpful to policy and decisionmakers for strategic decisions and can come to good use in techno-economic analysis of CCS. There are however two large drawbacks that limit the use of probabilistic methods as uncertainty analysis tools. The first relates to the need to describe the variability of the input parameters in probabilistic terms. It requires as a minimum a description of minimum and maximum, but preferably a full PDF. The problem, however, is that the exact values of parameters are often not known (recall the difference between uncertainty and variability, where in the latter case we often do have a good grasp of the variation of values). Randomly assigning probability distributions to inputs can result in misleading outputs. As a result, this would therefore not only not reduce uncertainty, but possibly generate a false sense of certainty and thereby lose its value for answering prognostic questions $[155,160]$. It could 
however still be useful for answering diagnostic questions or for calculating global sensitivity indicators. Given this consideration, we argue here that probabilistic uncertainty analysis is better suited to deal with variability, than with true uncertainty.

A second large drawback of, especially, Monte Carlo simulation is that it needs a large amount of runs to become a) statistically significant, and b) to estimate sensitivity indices ${ }^{22}$, see e.g. $[154,182]$. Such large amounts of runs could be feasible for simple and/or analytical models - like most economic models used in techno-economic analysis - but are a large obstacle for highly non-linear numerically solved models - like rigorous process models.

\subsection{Common pitfalls in probabilistic uncertainty analysis}

For probabilistic uncertainty analysis, the following additional pitfalls apply:

- Basic Monte Carlo simulation methods assume that input variables are independent of each other, which is often not the case in reality. Ignoring the correlation between dependent variables may lead to a false ranking of influential parameters. To address this issue, a dependence structure (known as a copula) can be created prior to sampling individual uncertain variables [174]. Alternatively, this problem can sometimes be avoided by explicitly modelling the relationship between two variables (e.g., depth and pressure or temperature in a subsurface reservoir model).

- The PDFs of input variables are poorly defined, or are insufficiently definable $[155,160]$. Monte Carlo simulation will compile seemingly reliable PDFs of model output regardless the quality of the input distributions. It may therefore create a false sense of certainty for the user of the sensitivity study, that could lead to wrong decisions being taken.

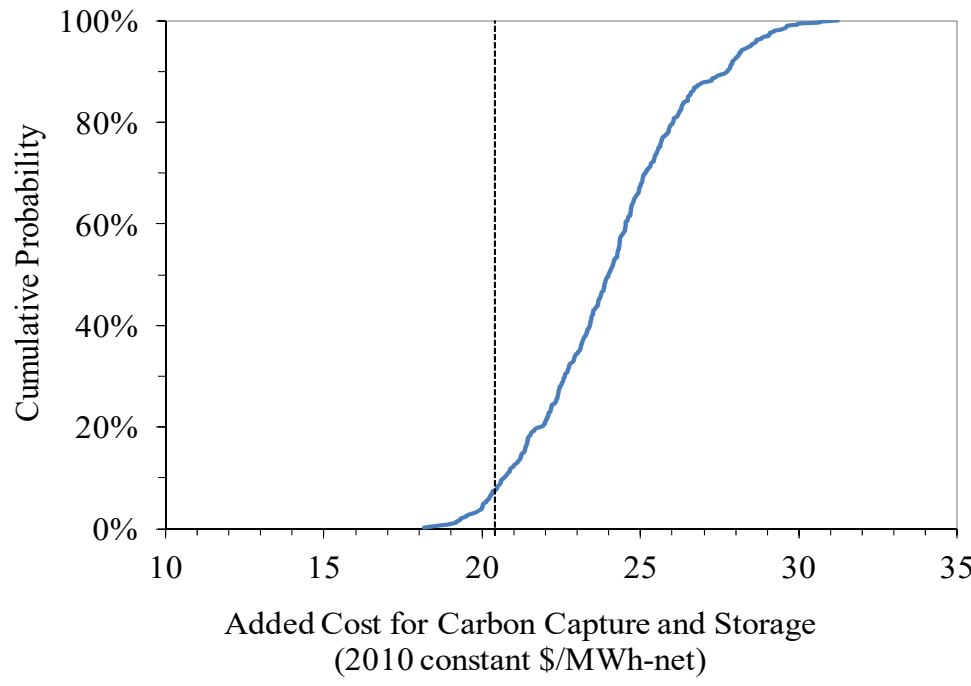

\footnotetext{
${ }^{22}$ For instance, when using a brute force method, the number of model runs $N$ required to calculate variance based sensitivity indicators (like the conditional mean) $E\left(Y \mid X_{i}\right)=1000$, then $N^{2}=1000^{2}$ runs would be required to calculate the sensitivity indices. Even with smart computational methods like Saltelli's [174], the amount of runs required is still $N(k+2)$ where $k$ is the number of variables to be varied. Linear regression on the Monte Carlo mapping generates sensitivity indicators at lower $\operatorname{cost} C=N$, but only calculates univariate (first order) sensitivity indicators (there are other post-processing methods that have this low cost but they have the same drawback, see e.g. [155]).
} 
Figure 3-4. Illustrative example of Monte Carlo simulation. Output of a Monte Carlo uncertainty analysis from [179].

Figure footnote: The figure shows both the deterministic (vertical dashed line, no uncertainty) and the probabilistic (solid line) values of the added cost of $\mathrm{CO}_{2}$ capture for a supercritical coal power plant under the constraint of a $1000 \mathrm{lb}$ of $\mathrm{CO}_{2} / \mathrm{MWh}$ gross emission (US) performance standard. For the assumptions of this study there is less than a $10 \%$ likelihood of realising the nominal (deterministic) value of added cost. The most likely cost (50\% probability) is $\$ 24.5 / \mathrm{MWh}$ (about $4 \$ / \mathrm{MWh}$ more than the deterministic value).

\subsubsection{Recommended practices for sensitivity analysis and probabilistic uncertainty analysis}

As the above sections have shown, sensitivity and probabilistic uncertainty analysis require the rational selection of parameters to vary, how to treat them, and which values, ranges, or PDFs to apply, to perform the uncertainty analysis in a meaningful and efficient manner.

A first good practice then, is to rationally choose the parameters to vary. Initial screening of parameter sensitivity can help reducing the number of varied parameters $k$ and makes the sensitivity analysis more efficient. Alternatively, one can find influential parameters for similar problems in academic or grey literature. When planning to run a Monte Carlo simulation for instance, local SA can be used to discard parameters that are not influential, reducing the required number of Monte Carlo runs. Care should be taken though to also test the sensitivity of parameters when interacting with others, before discarding them altogether. It is good practice to find a balance between the number of parameters to vary and their range. If the range is too wide and/or too many parameters are varied, the results may be less useful and relevant observations may get lost in the mass of output variance, apart from putting too much effort to answering the research question. Especially for probabilistic uncertainty analysis, when the chosen range is too small and/or too few parameters are varied, the results may not be global, thus inconclusive [174].

A second good practice is to explicitly distinguish between different types of input data (e.g. physical properties versus operational/design conditions/choices, measured data versus expert opinions, economic data versus technical data) or how to cluster them (e.g. a measured value times its weight) [174].

Finally, defining a good "sampling scheme" upfront can save a lot of hassle and unnecessary repetition. Sampling means the amount and values (or positions in a range) of the input data points that are chosen. Sampling can for instance be done by picking random values from a range of possible values, but this may lead to clusters of points and gaps. The modeller can also use random values within subintervals (called stratified sampling) or using particular values within subintervals (also stratified sampling) [174]. Especially for multiparameter sensitivity analysis, several sampling schemes have been developed, focussing on reducing the number of points that need to be evaluated, while remaining a required level of predictive power of the sensitivity analysis. Such sampling schemes are addressed elsewhere, e.g., [174] and include full factorial (only two levels of values: 1/1 or $\min / \max$ ); fractional factorial (FF, only two levels of values: $-1 / 1)$; Latin Hypercube (LH: multiple levels of values, stratified, needs number of simulations to be larger than the number of varied parameters ), or multivariate stratified sampling.

\subsubsection{Emerging uncertainty methods}

\subsubsection{Complementary analysis of qualitative uncertainties with pedigree analysis}

What: Pedigree analysis is a systematic and harmonised approach to identify and assess knowledge strength in order to minimise subjectivity and increase transparency. It is part of the NUSAP system (Numeral, Unit, Spread, Assessment, Pedigree) for uncertainty assessment and communication 
proposed by Ravetz \& Funtowicz [183]. Generally speaking, pedigree analysis provides an evaluation of the production process of information (how was it measured, derived, theorised?), and investigates the different aspects of the underpinning of the numbers and scientific status of the knowledge used [173], further explained below. Pedigree analysis has key advantages. For instance, it identifies the different sorts of uncertainty in quantitative information and enables them to be displayed in a standardized and self-explanatory way. It also allows to assess the quality of models by increasing transparency on the assumptions and choices and assessing uncertainties in the underlying knowledge base used for building up a process or cost model. Pedigree analysis is flexible in its use and can be used on different levels of comprehensiveness: from a rough sketch to a sophisticated procedure involving structured informed in-depth group discussions on a parameter-by-parameter format. Quite often results are used to develop so-called diagnostic diagrams, which are a convenient way to view each of the key parameters in terms of two crucial attributes: relative contribution to the sensitivity of the output and their strength. Finally, and possibly one of the key advantages, it fosters an enhanced appreciation of the issue of uncertainty in information.

How: Pedigree is expressed using a set of problem-specific criteria that serve to assess different aspects of knowledge strength. It is basically a systematic multi-criteria evaluation of the production process of knowledge, therefore looking not only at parameter data but also to knowledge available when building up a model. For example, commonly used criteria are proxy (is something measured directly, or is it estimated using an indirect indicator?) and theoretical understanding (is the data or model based on a well-established scientific theory, or crude speculation?). The type and number of criteria should be tailored to the specific situation.

Assessment of pedigree requires qualitative expert judgment. Expert elicitation systematically makes explicit use of unwritten insights 'in the heads of experts' (e.g., the modeler, or a group of experts), focusing on limitations, strengths, and weaknesses of the available knowledge base. To minimise subjectivity and arbitrariness in the evaluation, a pedigree matrix is used to systematically and transparently transform expert judgment into a numerical scale. A pedigree matrix (Table 3-4) is basically a table with the criteria as columns, and strength scores (typically 0 (weak) to 4 (strong)) as the rows (note that also other scales can be used). In each cell of the table, a linguistic description is provided with the requirements that should be met to receive a particular score. These descriptions therefore serve as yardsticks. Note that these linguistic descriptions are mainly meant to provide guidance in attributing scores to each of the criteria for a given parameter, and as such the descriptions should be tailored to the focus of the study. How clear, explicit and tailormade to the problem the linguistic definitions are, is a key component of the proper and successful application of pedigree matrices. A total score can be then produced for a given parameter or (sub)model (see Figure 3-6). This, however, requires that the modeler (or the experts) weighs the different criteria of the pedigree matrix, for instance by deciding that all criteria are equally important or that some criteria are more important than others. Furthermore, when a group of experts is scoring, total scores of each expert can be aggregated using the median of the expert respondents' scores. Interquartile ranges can then be used as an indicator of inter-expert heterogeneity (degree of consensus/disagreement in the strength of the knowledge base for a given parameter/model). Table 3-4 provides an example of a typical pedigree matrix used to assess the strength of data in a cost assessment of $\mathrm{CO}_{2}$ capture technologies. 
Table 3-4. Example of a pedigree matrix used in the EDDiCCUT project to assess the strength of data input for cost assessment [184].

\begin{tabular}{|c|c|c|c|c|c|}
\hline SCORE & Proxy & Reliability of source & $\begin{array}{l}\text { Completeness (only for } \\
\text { equipment list) }\end{array}$ & $\begin{array}{l}\text { Completeness (all other } \\
\text { parameters) }\end{array}$ & Validation process \\
\hline 4 & $\begin{array}{l}\text { A direct measure } \\
\text { of the desired } \\
\text { quality }\end{array}$ & $\begin{array}{l}\text { Measured/official } \\
\text { industrial, vendor, } \\
\text { and/or supplier data } \text { da }^{23}\end{array}$ & $\begin{array}{l}\text { Representative data for } \\
\text { all line items (processes, } \\
\text { instruments, electro, } \\
\text { civil, mechanical, etc.) }\end{array}$ & $\begin{array}{l}\text { Complete data from a large } \\
\text { number of samples over a } \\
\text { representative period }\end{array}$ & $\begin{array}{lr}\text { Compared } & \text { with } \\
\text { independent data from } \\
\text { similar systems that } \\
\text { have been built }\end{array}$ \\
\hline 3 & $\begin{array}{l}\text { Good fit to } \\
\text { measure }\end{array}$ & $\begin{array}{l}\text { Qualified estimate by } \\
\text { industrial } r \text { expert } \\
\text { supported by industry } \\
\text { data }\end{array}$ & $\begin{array}{l}\text { Representative data for } \\
\text { all process equipment } \\
\text { (equipment list, heat and } \\
\text { mass balance, PFD) }\end{array}$ & $\begin{array}{l}\text { Complete data from a large } \\
\text { number of samples but for } \\
\text { unrepresentative periods or } \\
\text { from representative periods but } \\
\text { for a small number of samples }\end{array}$ & $\begin{array}{l}\text { Compared with } \\
\text { independent data of } \\
\text { similar systems that } \\
\text { have not been built }\end{array}$ \\
\hline 2 & $\begin{array}{l}\text { Correlated but } \\
\text { does not measure } \\
\text { the same thing }\end{array}$ & $\begin{array}{l}\text { Reviewed data derived } \\
\text { from independent open } \\
\text { literature }\end{array}$ & $\begin{array}{l}\text { Representative data for } \\
\text { most important process } \\
\text { equipment (equipment } \\
\text { list, heat and mass } \\
\text { balance, PFD) }\end{array}$ & $\begin{array}{l}\text { Almost complete data but from } \\
\text { a small number of samples or for } \\
\text { unrepresentative periods or } \\
\text { incomplete data from adequate } \\
\text { number of samples and periods }\end{array}$ & $\begin{array}{l}\text { Validation } \\
\text { measurements are not } \\
\text { independent, include } \\
\text { proxy variables or have } \\
\text { a limited domain }\end{array}$ \\
\hline 1 & $\begin{array}{l}\text { Weak correlation } \\
\text { but commonalities } \\
\text { in measure }\end{array}$ & $\begin{array}{l}\text { Non-reviewed data } \\
\text { from open literature }\end{array}$ & $\begin{array}{l}\text { Data from an adequate } \\
\text { number of process } \\
\text { parameters eat and mass } \\
\text { balance, PFD) }\end{array}$ & $\begin{array}{l}\text { Almost complete data but from } \\
\text { a small number of samples and } \\
\text { unrepresentative periods }\end{array}$ & $\begin{array}{l}\text { Weak and indirect } \\
\text { validation }\end{array}$ \\
\hline $\mathbf{0}$ & $\begin{array}{l}\text { Not correlated and } \\
\text { not clearly related }\end{array}$ & $\begin{array}{l}\text { Non-qualified estimate } \\
\text { or unknown origin }\end{array}$ & $\begin{array}{l}\text { Only high level or } \\
\text { incomplete } \\
\text { available }\end{array}$ & $\begin{array}{l}\text { Incomplete data from a small } \\
\text { number of samples for an } \\
\text { unrepresentative period }\end{array}$ & $\begin{array}{l}\text { No } \\
\text { performed }\end{array}$ \\
\hline
\end{tabular}

Proxy: refers to how good or close a measure of the quantity that is modelled is to the actual quantity one wants to score. Reliability of source: evaluates the origin of the collected data. Completeness: this criterion assesses the coverage of the data, taking into account the information reported for process inputs, outputs, and associated stressors. It considers not only the amount but also the specific relevance of the presented data. Validation: refers to the degree to which data and assumptions used to produce the numeral of the parameter has been cross-checked against independent sources.

Results of the pedigree analysis can be combined with those of a sensitivity analysis in a diagnostic diagram. The diagnostic diagram (Figure 3-5) is based on the notion that neither spread alone (as obtained from a sensitivity analysis) nor strength alone (as obtained from the pedigree score) is a sufficient measure for assessing the quality of a model output. Robustness of model output to parameter strength could be good even if parameter strength is low because the importance of that parameter for the final results may be minor. Alternatively, robust conclusions can be derived for parameters that have large impact on output spread and high parameter strength. Mapping components of the knowledge base in a diagnostic diagram thus reveals the weakest spots and helps in the setting of priorities for improvement.

\footnotetext{
${ }^{23}$ Note that vendor or supplier data was in this example score the highest regarding reliability. This of course does not need to be the case, and while carrying out the scoring the modeler (or expert) should take into account whether the data comes from an offer or from a performance guarantee in a contract, the letter being much more reliable.
} 


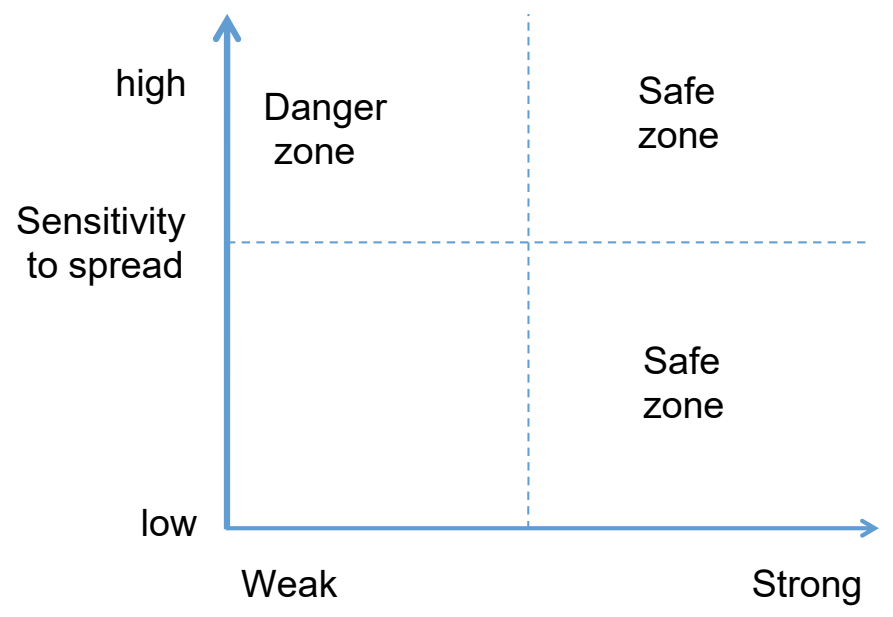

\section{Pedigree \\ Figure 3-5. Example of a diagnostic diagram for the evaluation of the robustness of the model and model parameters.}

Figure footnote: The so-called danger zone is the quadrant containing parameters to which the model output is highly sensitive, but which have a weak pedigree, i.e., the results are dependent on a parameter that is characterized with a weak knowledge base. The safe zones are the two quadrants containing parameters that have a high pedigree. Parameters in the zone characterized with weak pedigree-low sensitivity should be further examined as the low sensitivity to spread could be caused by the assumptions in the knowledge driven by a weak knowledge base.

When: Pedigree analysis can be used for any process or cost evaluation as it increases transparency in the reporting of quality of the data and models used in a given assessment. Such information is important for understanding the robustness of model outcomes as well as to facilitate discussion in expert groups. Furthermore, the use of, e.g., diagnostic diagrams allows for easier representation of the quality and importance of uncertainties. The added value of the approach is especially significant for assessments where there is a weak knowledge base. For instance, technology and costs assessments of technologies that are currently at low TRL level, for which only partial information is available, and significant number of assumptions need to be made regarding performance and scaling.

\begin{tabular}{|c|c|c|c|c|c|}
\hline $\begin{array}{l}\text { Pedigree scores for technical } \\
\text { input data }\end{array}$ & Proxy & $\begin{array}{l}\text { Empirical } \\
\text { basis }\end{array}$ & $\begin{array}{c}\text { Theoretical } \\
\text { understanding }\end{array}$ & $\begin{array}{c}\text { Methodological } \\
\text { rigour }\end{array}$ & $\begin{array}{c}\text { Validation } \\
\text { process }\end{array}$ \\
\hline \multicolumn{6}{|l|}{ SA 2a: $\mathrm{H}_{2}$ unit without $\mathrm{CO}_{2}$ capture } \\
\hline Feed streams & 4 & 3 & 4 & 3 & - \\
\hline Design parameters & 3 & 3 & 4 & 4 & 3 \\
\hline Boundary conditions & 4 & 2 & 4 & 3 & 3 \\
\hline Other physical properties & 4 & 4 & 4 & 4 & 3 \\
\hline \multicolumn{6}{|l|}{ SA 2b: $\mathrm{CO}_{2}$ capture unit } \\
\hline Feed streams & 4 & 3 & 4 & 3 & 3 \\
\hline Design parameters & 3 & 2 & 3 & 3 & 2 \\
\hline Boundary conditions & 4 & 2 & 4 & 3 & 3 \\
\hline Other physical properties & 4 & 4 & 4 & 4 & 3 \\
\hline \multicolumn{6}{|l|}{ SA 4a: Conventional polyol } \\
\hline Feed streams & 4 & 3 & 4 & 3 & - \\
\hline Design parameters & 3 & 3 & 3 & 2 & 1 \\
\hline Boundary conditions & 4 & 2 & 4 & 3 & 3 \\
\hline Other physical properties & 4 & 3 & 4 & 3 & 1 \\
\hline \multicolumn{6}{|l|}{ SA 4b: $\mathrm{CO}_{2}$-based polyol } \\
\hline Feed streams & 4 & 3 & 4 & 2 & - \\
\hline Design parameters & 3 & 2 & 3 & 2 & 1 \\
\hline Boundary conditions & 4 & 2 & 4 & 3 & 3 \\
\hline Other physical properties & 4 & 2 & 4 & 2 & 0 \\
\hline
\end{tabular}




\begin{tabular}{|c|c|c|c|c|c|}
\hline $\begin{array}{l}\text { Pedigree scores } \\
\text { for economic } \\
\text { input data }\end{array}$ & Proxy & Reliability of source & $\begin{array}{l}\text { Completeness (only } \\
\text { equipment) }\end{array}$ & $\begin{array}{l}\text { Completeness (other } \\
\text { parameter) }\end{array}$ & Validation process \\
\hline \multicolumn{6}{|c|}{ SA 1: Naphtha production and transport } \\
\hline CAPEX & - & - & - & - & - \\
\hline OPEX & 4 & 4 & - & 3 & 0 \\
\hline \multicolumn{6}{|c|}{ SA 2a: $\mathrm{H}_{2}$ unit without $\mathrm{CO}_{2}$ capture } \\
\hline CAPEX & 4 & 2 & 2 & 3 & 3 \\
\hline OPEX & 4 & 3 & - & 2 & 0 \\
\hline \multicolumn{6}{|c|}{ SA 2b: $\mathrm{CO}_{2}$ capture unit } \\
\hline CAPEX & 4 & 2 & 2 & 3 & 2 \\
\hline OPEX & 4 & 3 & - & 2 & 0 \\
\hline \multicolumn{6}{|c|}{$\mathrm{SA}$ 3: $\mathrm{CO}_{2}$ transport and storage } \\
\hline CAPEX & 4 & 2 & 1 & 3 & 1 \\
\hline OPEX & 4 & 2 & - & 2 & 1 \\
\hline \multicolumn{6}{|c|}{ SA 4a: Conventional polyol } \\
\hline CAPEX & 4 & 2 & 1 & 3 & 1 \\
\hline OPEX & 4 & 3 & - & 2 & 0 \\
\hline \multicolumn{6}{|c|}{ SA 4b: $\mathrm{CO}_{2}$-based polyol } \\
\hline CAPEX & 4 & 2 & 1 & 3 & 1 \\
\hline OPEX & 4 & 3 & - & 2 & 0 \\
\hline \multicolumn{6}{|l|}{ SA 5: Chemicals } \\
\hline CAPEX & - & - & - & - & - \\
\hline OPEX & 3 & 3 & - & 2 & 0 \\
\hline
\end{tabular}

Figure 3-6. Illustrative example of the use of pedigree analysis.

Figure footnote: In [184], pedigree matrices were used in the evaluation of the techno-economic and environmental performance of carbon capture at a hydrogen unit in a refinery combined with $\mathrm{CO}_{2}$ conversion into polyol synthesis. As illustrated, the figures above show pedigree matrices for input data (for the technical model and the cost assessment). The information is provided at the aggregated level of a System Area (each representing group of processes that are part of the value chain under study). Note that the criteria selected for each matrix has been tailored to each type of assessment, for instance, CAPEX and OPEX when assessing data used for costing, and feed streams, design parameters, boundary conditions, and other physical properties for technical input data. Together with the matrix, the article describes the reasoning behind the choice of scores (which was done by the authors).

\subsubsection{Pseudo statistical approach}

What: This approach has been developed in the realm of Life Cycle Assessment (including life cycle costing, see e.g., [185]) but it can be applied to other fields including TEA. The methodology aims to enable the use of Monte Carlo analysis to assess the propagation of uncertainty and/or variability introduced by both uncertainties in data and methodological choices into the final results. An example of a methodological choice relevant to this guideline is the allocation of the costs of capture when more than one valuable product is produced in a given system (e.g., polygeneration systems). The key feature of this method (compared to others) is that it explicitly acknowledges large choice related uncertainties on top of parameter uncertainties.

Compared to the natural variability of data, which could be represented through a probability distribution, there is not natural variability in a discrete choice (such as allocation). Although the methodology treats both data and methodological choices in a similar way, the use of the term "pseudo statistical" has the goal of tacitly acknowledging that the use of terminology (e.g. probability, statistical) is not entirely suitable for all cases. 
How: To be able to introduce pseudo-statistical propagation of a methodological choice, a variable called methodological preference (p) (as percentage) is introduced. If there is only one methodological choice, $\mathrm{p}$ equals $100 \%$, but if there are different choices (for instance allocating the capture costs based on energy (method 1), mass (method 2), price of the products (method 3)) then each choice is assigned different $p$ values (each between 0 and 100\%), with the condition that the sum of all $\mathrm{p}$ values equals $100 \%$. In this respect, the pseudo statistical approach is very similar to assigning weights in multi-criteria analysis. As the p values reflect preferences, they are inherently subjective. The methodology therefore allows to explicitly include values (preferences) of stakeholders or to include values that represent trends found in e.g., literature. Compared to other methods, the main advantage is on selecting $\mathrm{p}$ values that reflect stakeholder preference or preferences. If literature is used instead, other methods could be used (e.g., analysing discrete cases). The methodological values provided by the experts define the ranges of methodological preferences, such that for each range one methodological choice takes place. The value of a random number from a uniform distribution between 0 and 100 is then generated and evaluated for the ranges of preferences. In mathematical terms, this can be represented as follows:

$$
\text { Methodological choice }=\left\{\begin{array}{r}
\text { method } 1 \text { if } x \in\left[0, p_{1}\right] \\
\text { method } 2 \text { if } x \in\left[p_{1}, p_{1}+p_{2}\right] \\
\text { method } 3 \text { if } x \in\left[p_{1}+p_{2}, p_{1}+p_{2}+p_{3}\right] \\
\vdots \\
\text { method } n \text { if } x \in\left[p_{1}+p_{2}+p_{3}+\cdots+p_{n-1}, 100\right] \\
x \sim U(0,100)
\end{array}\right.
$$

After the parameters are defined, Monte Carlo analysis can then be used for the random sampling to propagate the uncertainty. In the results, the uncertainty introduced by different combinations of methodological choices can then be included. This approach accounts in a pseudo-statistical manner for a representative sample of combinations of methodological choices.

When: The method is particularly recommended when a large number of methodological choices are required (for instance in process modelling and/or costs assessment) thereby avoiding the need for developing one-at the time scenario modelling for choice-related uncertainties, which depending on the number of methodological choices in a given analysis, can become easily very time and resourceintensive.

\subsubsection{Reduced order models for global uncertainty analysis}

What: As mentioned above in section 3.3.3.2, probabilistic uncertainty and sensitivity analysis comes at the expense of many thousands (or more) of model realisations. This may severely hamper their use for computationally heavy models, such as most first principles process models, especially when high-performance computing is unavailable. Therefore, the discipline of global uncertainty analysis has moved towards the use of reduced order models $\hat{g}$ (ROMs, also meta-models, or surrogate models) as a representation of heavy numerical models:

$$
\mathbf{y}=g(\mathbf{x}) \approx \hat{g}(\mathbf{x})=\hat{g}(\mathbf{v}, \boldsymbol{\theta})
$$

Where, $\mathbf{v}$ is a vector of deterministic input parameters and $\boldsymbol{\theta}$ is a set of stochastic input parameters. Different methods of model reduction have been studied and some have found their way to the CCS field of research: Hanak and others have used Artificial Neural Network (ANN) models as surrogates for full physical calcium looping models, and have used these to perform integrated global uncertainty 
analysis of the economics of calcium looping systems [159, 186]. Sun et al. [187] used Polynomial Chaos Expansion (PCE) to produce surrogate models of physical models for $\mathrm{CO}_{2}$ storage well leakage and combined those with risk and cost analysis tools to retrieve stochastic integrated leakage risk and cost information.

How: The reduced order model types for global uncertainty analysis vary, but they all rely on the same approach:

1. Run a limited number (typically several hundreds) of realisations of the full physical model, with uncertainty and/or variability assigned to its inputs (i.e. running different realisations for different combinations of model inputs from predefined parameter ranges).

2. Fit an analytical (e.g. polynomial, ANN, linear regression) reduced order model based on this limited set of model runs.

3. Perform a full Monte Carlo simulation on the ROM. Given that the ROM is analytical, this should take a fraction of the time of a full physical model.

4. Calculate the sensitivity indicators, either directly/analytically when possible or based on the Monte Carlo runs.

The methods to produce some reduced order models and to calculate sensitivity indicators from their probabilistic uncertainty analysis can be quite mathematical and therefore can easily seem daunting to the general techno-economic analysis practitioner. But since the basis of these methods is now well established, also more accessible, ready to use kind of tools are coming available (see section 3.5), which should open up these methods to a wider audience.

When: Previous studies have shown that the used reduced order models are very accurate in describing the input-output combinations of the original physical model within the specified ranges of application [154]. They are therefore very useful when reliable representations of uncertainty and variability are necessary to answer what will kind of questions using Monte Carlo simulation, while the original model would be too heavy (computationally intensive) to allow this. They are especially helpful to perform global sensitivity analysis of heavy models, and to aid in factor prioritisation and factor fixing. Like with any probabilistic method however, they are best suited for the variability of input parameters, and less for real uncertainty, given that there may not be a reasonable basis for probability density functions of truly uncertain parameters.

Table 3-5 and Table 3-6 and Figure 3-7 exemplify the use of reduced order models for global sensitivity analysis and the calculation of Variance based (Sobol) sensitivity indicators. The example is based on a series of papers [188-190] in which surrogate models of an MEA capture plant were built based on a full process model and compared with process data from the National Carbon Capture Center (NCCC) in Alabama, USA [191]. Table 3-5 shows the names of the varied parameters along with sources that contain more details of the sub-model development and uncertainty quantification. 
Table 3-5. List of parameters for MEA model considered in uncertainty analysis.

\begin{tabular}{cc}
\hline Parameter Number & Parameter Name \\
\hline 1 & Thermodynamic Model (Aspen Plus names in brackets) [189] \\
2 & Gibbs energy of formation at infinite dilution (DGAQFM) for MEA+ \\
3 & Gibbs energy of form. at infinite dilution (DGAQFM) for MEACOO- \\
4 & Enthalpy of formation at infinite dilution (DHAQFM) for MEA+ \\
5 & Enthalpy of formation at infinite dilution (DHAQFM) for MEACOO- \\
6 & Henry parameter A (HENRY/1) for MEA- $\mathrm{H}_{2} \mathrm{O}$ \\
7 & Henry parameter B (HENRY/2) for MEA- $\mathrm{H}_{2} \mathrm{O}$ \\
8 & NRTL binary interaction parameter A (NRTL/1) for MEA-H $\mathrm{H}_{2} \mathrm{O}$ \\
9 & NRTL binary interaction parameter A (NRTL/1) for $\mathrm{H}_{2} \mathrm{O}-\mathrm{MEA}$ \\
& NRTL binary interaction parameter B (NRTL/2) for $\mathrm{H}_{2} \mathrm{O}-\mathrm{MEA}$ \\
\hline 10 & Holdup Model [190] \\
\hline 11 & $\mathrm{H}_{\mathrm{L} 1}$ (liquid holdup) \\
\hline 12 & $\mathrm{H}_{\mathrm{L} 2}$ (liquid holdup) \\
\hline 13 & $\mathrm{~A}_{1}$ (interfacial area) \\
\hline
\end{tabular}

A total of 23 steady-state data sets were generated during the test campaign at NCCC; the propagation of uncertainty was demonstrated for three cases in the previous work [191] and for two additional cases in this paper. Table 3-6 shows the key absorber operating variables for the two cases; the case labels are consistent with the previous work.

Table 3-6. Key operating variables for absorber simulation for two cases

\begin{tabular}{lcc}
\hline \multicolumn{1}{c}{ Variable } & Case K2 & Case K7 \\
\hline Lean Solvent Mass Flowrate $(\mathrm{kg} / \mathrm{hr})$ & 11794 & 11791 \\
Flue Gas Flowrate $(\mathrm{kg} / \mathrm{hr})$ & 2243 & 2233 \\
Lean Solvent Loading (mol CO$/ \mathrm{mol} \mathrm{MEA)}$ & 0.247 & 0.399 \\
Lean Solvent MEA Weight Fraction (g MEA/g [MEA+ $\left.\left.\mathrm{H}_{2} \mathrm{O}\right]\right)$ & 0.312 & 0.288 \\
$\mathrm{CO}_{2}$ Mole Percent in Flue Gas & 11.40 & 9.18 \\
Percent Capture of $\mathrm{CO}_{2}$ & 99.49 & 54.76 \\
\hline
\end{tabular}

The main advantage of developing surrogate models, is the lower computational effort, and therefore the ability to calculate variance-based sensitivity indicators. In this example, this was done in the software platform PSUADE ([192], more information in section 3.5), which allows to calculate Sobol indices [193], which represent a decomposition of variance technique for determining the relative influence of each parameter on the model output. The development of the surrogated model required 1000 runs in Aspen Plus, taking 2-3 hours on a normal desktop machine. Based on these full model realisations, the reduced order model was fitted with Multivariate Adaptive Regression Splines (MARS) [194]. This reduced order model was then run to calculate the normalised Sobol indices in Figure 3-7 for the contributions of the parameters listed in Table 3-5. 

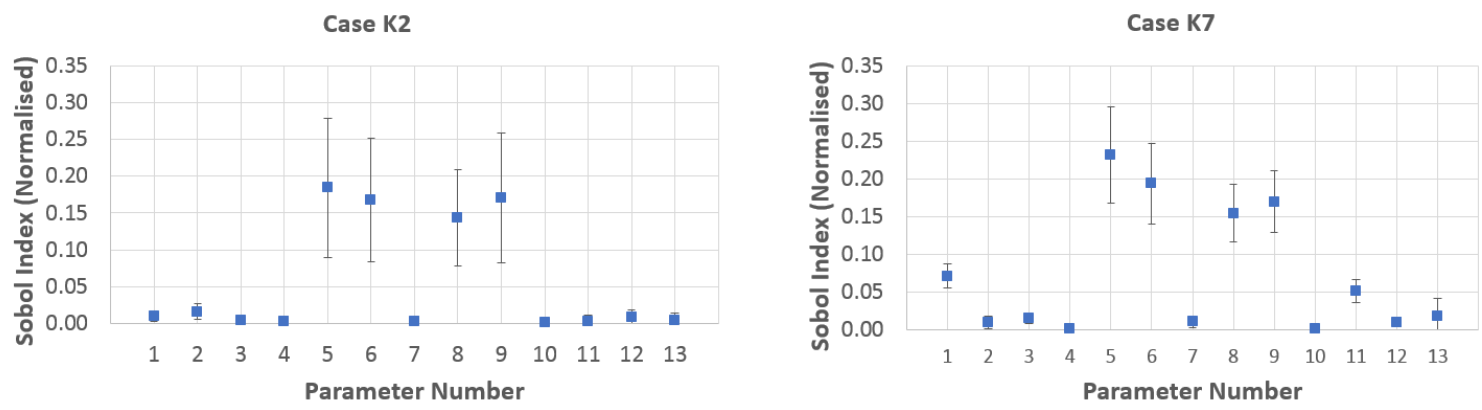

Figure 3-7. Normalised Sobol indices for contributions of individual parameters for two model simulation cases. Points represent mean values and error bars \pm 1 standard deviation $(n=100$ replications performed). Parameter numbers are consistent with those given in Table 3-1

Although the results demonstrate some spread in the values of Sobol indices that results from replicating the calculation, the relative importance is shown to be predicted consistently; for both cases parameters 5-6 and 8-9 are shown to have the highest effect on the $\mathrm{CO}_{2}$ capture prediction. For Case K2, the packing-related parameters (No. 10-13) are shown to have very minimal effect on the prediction of $\mathrm{CO}_{2}$ capture. For Case $\mathrm{K} 7$, this is not so due to the much higher loading of $\mathrm{CO}_{2}$ in the inlet solvent, which results in inefficient absorber operation. Although some of the thermodynamic model parameters still have the highest Sobol indices, some of the packing dependent parameters have higher values in comparison to Case $\mathrm{K} 2$. As a result, the calculated value of $\mathrm{CO}_{2}$ capture percentage is not insensitive to the parametrisation of the packing models (mass transfer, interfacial area, hydraulics).

\subsubsection{Strengths, weaknesses, and applicability of uncertainty methods to TEA}

The previous sections discussed a selection of uncertainty analysis methods available to the CCS techno-economic practitioner aiming to provide basic information on their use. Here, we synthesise this discussion into guidelines on choosing from these methods.

The choice for an uncertainty method depends first and foremost on the purpose of the uncertainty analysis. Recall that we divided these into diagnostic, prognostic, or factor prioritisation/factor fixing and model testing. Additionally, the choice will depend on such criteria including:

- The computational cost of running the model and uncertainty analysis.

- The number and type of input factors that need varying.

- The context of the analysis including the audience.

Note that selection criteria will differ for each user, and also depend on the purpose of the uncertainty analysis, as well as on their experience. Table 3-7 summarises the uncertainty analysis methods in terms of possible selection criteria and suggests TEA areas to which a method may be more or less applicable, while Figure 3-8 provides a general workflow for selecting uncertainty analysis methods. For diagnostic, or "what if" type of questions, local sensitivity analyses often suffice. They present a simple and clear picture of how an output might change as a result of varying one, or multiple parameters, e.g. how the performance and cost may vary as a result of changes in ambient conditions. We recommend the use of one-way or N-way sensitivity analysis, because they provide more information (on non-linear responses) than a standard OAT analysis.

For prognostic, or "what will" type of questions, we strongly recommend global uncertainty methods, provided that the input parameter probability can be quantified satisfactorily. If this is not the case, it 
is recommended to avoid prognostic analysis altogether, because there is a danger of creating a false sense of certainty (i.e., the receiver of the model output will see a carefully compiled probability density function, adding to the perception of certainty). However, if the probabilistic output is based on a poor description of input uncertainties, the result is still very weak and may be misleading. The requirement of quantifiable input PDF's implies that what will type of questions should preferably only be answered for CCS technologies and systems that have reached a certain level of advancement, arguably beyond the large pilot plant stage.

For both diagnostic and prognostic questions, we recommend complementing the quantitative uncertainty analysis with a qualitative one, like pedigree analysis. This is especially the case when the model and its results are used for policy and/or decision making, because it provides decision makers with information on how robust the model results are, which is indispensable for making informed decisions.

In case of factor prioritisation or factor fixing, sensitivity analysis is required. In the case of very simple (mostly linear) models without many parameter interactions, a local sensitivity method could suffice, where it is arguably advisable to go beyond OAT. However, factor prioritisation and fixing are often most needed in complex models, in which case a global (probabilistic) sensitivity analysis is essential, to also account for second or higher order effects of an input parameter on model output. If the purpose of the uncertainty analysis is model testing, we recommend using single (OAT) or multiple (NAT) parameter local uncertainty analysis. Preferably, the model is subjected to (combinations of) an extreme value of input parameter(s), that should trigger an extreme output (think e.g., of an extremely high cost of energy, which should lead to a very high cost of $\mathrm{CO}_{2}$ avoided).

Finally, for a non-specialist audience and sometimes even for a specialized audience, it is arguably a poor idea to show results of uncertainty analysis in very advanced (complex) graphs. To best convey the results, simple plots like tornado or spider plots may be more useful than more complex and less commonly used visualizations, e.g., scatter clouds. 
Table 3-7. Uncertainty analysis selection guide.

\begin{tabular}{|c|c|c|c|c|c|c|c|}
\hline $\begin{array}{l}\text { Possible selection } \\
\text { criteria }\end{array}$ & $\begin{array}{l}\text { OAT sensitivity } \\
\text { analysis }\end{array}$ & $\begin{array}{l}\text { One-way } \\
\text { sensitivity } \\
\text { analysis }\end{array}$ & $\mathrm{N}$-ways sensitivity analysis & $\begin{array}{l}\text { Global uncertainty } \\
\text { analysis (incl. Monte } \\
\text { Carlo) }\end{array}$ & Pedigree analysis & $\begin{array}{l}\text { Pseudo statistical } \\
\text { approach }\end{array}$ & $\begin{array}{l}\text { Reduced order global } \\
\text { uncertainty analysis }\end{array}$ \\
\hline $\begin{array}{l}\text { Purpose of } \\
\text { uncertainty analysis }\end{array}$ & $\begin{array}{l}\text { Understand first } \\
\text { order relative } \\
\text { ranking/importance } \\
\text { of parameters, } \\
\text { understand } \\
\text { response to } \\
\text { extreme input } \\
\text { values for single } \\
\text { parameters (model } \\
\text { testing) }\end{array}$ & $\begin{array}{l}\text { All of OAT } \\
\text { plus } \\
\text { understand } \\
\text { break points, } \\
\text { non- } \\
\text { linearities }\end{array}$ & $\begin{array}{l}\text { All previous plus } \\
\text { investigates interactions } \\
\text { and response to extremes } \\
\text { of multiple parameters }\end{array}$ & $\begin{array}{l}\text { To quantify the } \\
\text { probabilistic } \\
\text { variability/uncertainty } \\
\text { in the knowledge base } \\
\text { and how that impacts } \\
\text { an outcome. To foster } \\
\text { an understanding of the } \\
\text { distribution of } \\
\text { uncertainty and/or } \\
\text { variability. To quantify } \\
\text { the chance of a certain } \\
\text { event of happening. To } \\
\text { quantify the global } \\
\text { sensitivity to input } \\
\text { parameters }\end{array}$ & $\begin{array}{l}\text { To foster an } \\
\text { understanding of the } \\
\text { strength of the } \\
\text { knowledge base } \\
\text { underlying data or a } \\
\text { model }\end{array}$ & $\begin{array}{l}\text { To understand the } \\
\text { significance of } \\
\text { value-laden choices } \\
\text { compared to } \\
\text { parameter variation } \\
\text { on the model } \\
\text { outputs. }\end{array}$ & $\begin{array}{l}\text { To quantify the } \\
\text { probabilistic } \\
\text { variability/uncertainty of } \\
\text { computationally heavy } \\
\text { models in our knowledge } \\
\text { and how that impacts an } \\
\text { outcome. To foster an } \\
\text { understanding of the } \\
\text { distribution of uncertainty } \\
\text { and/or variability. To } \\
\text { quantify the chance of a } \\
\text { certain event of } \\
\text { happening. To quantify } \\
\text { the global sensitivity to } \\
\text { input parameters }\end{array}$ \\
\hline $\begin{array}{l}\text { Relative } \\
\text { computational } \\
\text { cost/time }\end{array}$ & Low & Low & Medium & Medium - Very High & $\begin{array}{l}\text { Not applicable, but } \\
\text { requires substantial } \\
\text { time for expert } \\
\text { elicitation }\end{array}$ & Medium - Very High & Medium \\
\hline Pre-processing data & low & Low & Low & high & medium & High & High \\
\hline $\begin{array}{l}\text { Type of uncertainty } \\
\text { method }\end{array}$ & quantitative & Quantitative & Quantitative & quantitative & qualitative & $\begin{array}{l}\text { qualitative \& } \\
\text { quantitative }\end{array}$ & quantitative \\
\hline $\begin{array}{l}\text { Able to cope with } \\
\text { interactions }\end{array}$ & No & No & Yes & Yes & Not applicable & Yes & Yes \\
\hline $\begin{array}{l}\text { Able to cope with } \\
\text { non-linearity }\end{array}$ & No & Yes & Yes & Yes & Not applicable & Yes & Yes \\
\hline Target user/ reader & General public & $\begin{array}{l}\text { General } \\
\text { public }\end{array}$ & $\begin{array}{l}\text { Depends on number of } \\
\text { investigated parameters } \mathrm{N} \\
\text { (the larger the number } \\
\text { the more specialized } \\
\text { knowledge is required to } \\
\text { interpret the results) }\end{array}$ & Specialist & General public & Specialist & Specialist \\
\hline
\end{tabular}




\begin{tabular}{|c|c|c|c|c|c|c|c|}
\hline \multicolumn{8}{|c|}{ Table 3-7. (cont'd) } \\
\hline $\begin{array}{l}\text { Prior knowledge } \\
\text { required on } \\
\text { parameters to } \\
\text { conduct the } \\
\text { assessment }\end{array}$ & Low & Medium & Medium & High & $\begin{array}{l}\text { Understanding the } \\
\text { knowledge base is the } \\
\text { goal of this type of } \\
\text { assessment }\end{array}$ & High & High \\
\hline $\begin{array}{l}\text { Example TEA areas } \\
\text { applicable to }\end{array}$ & $\begin{array}{l}\text { Linear cost models, } \\
\text { e.g., capital cost } \\
\text { factor models }\end{array}$ & $\begin{array}{l}\text { Simple } \\
\text { technical and } \\
\text { cost } \\
\text { performance } \\
\text { models } \\
\text { without } \\
\text { strong } \\
\text { interactions } \\
\text { between } \\
\text { parameters, } \\
\text { e.g., } \\
\text { exponent } \\
\text { models for } \\
\text { capital costs }\end{array}$ & $\begin{array}{l}\text { More complex technical } \\
\text { and cost models where } \\
\text { there are known } \\
\text { parameter interactions } \\
\text { and non-linearities, e.g. } \\
\text { rigorous/first principle } \\
\text { process models }\end{array}$ & $\begin{array}{l}\text { More complex technical } \\
\text { and cost models where } \\
\text { there is enough data to } \\
\text { make/select PDF's, e.g., } \\
\text { NPV and LCOE models }\end{array}$ & $\begin{array}{l}\text { Any, particularly TEA } \\
\text { studies used for policy } \\
\text { and decision making } \\
\text { and where knowledge } \\
\text { is contested or } \\
\text { inherently limited (low } \\
\text { TRL) }\end{array}$ & $\begin{array}{l}\text { Situations where } \\
\text { contextual choices } \\
\text { are expected to } \\
\text { have an impact. }\end{array}$ & $\begin{array}{l}\text { Highly complex and non- } \\
\text { linear process models that } \\
\text { are to be directly linked } \\
\text { with economic estimates }\end{array}$ \\
\hline $\begin{array}{l}\text { Limitations } \\
\text { (preferably not to } \\
\text { be used for) }\end{array}$ & $\begin{array}{l}\text { When strong non- } \\
\text { linearities and } \\
\text { interactions are } \\
\text { expected }\end{array}$ & $\begin{array}{l}\text { When strong } \\
\text { interactions } \\
\text { between } \\
\text { parameters } \\
\text { are expected }\end{array}$ & $\begin{array}{l}\text { When the process models } \\
\text { are computationally very } \\
\text { heavy }\end{array}$ & $\begin{array}{l}\text { When there is too little } \\
\text { information on the } \\
\text { shape and potential } \\
\text { values of PDF's. } \\
\text { When the model is } \\
\text { computationally heavy }\end{array}$ & $\begin{array}{l}\text { When a large set of } \\
\text { input data needs to be } \\
\text { analysed }\end{array}$ & $\begin{array}{l}\text { When there is too } \\
\text { little information on } \\
\text { the shape and } \\
\text { potential values of } \\
\text { PDF's. When the } \\
\text { model is } \\
\text { computationally } \\
\text { heavy. When there } \\
\text { is a limited (or non- } \\
\text { existing) number of } \\
\text { value-laden } \\
\text { choices. }\end{array}$ & $\begin{array}{l}\text { Models that do not need } \\
\text { reduction to become } \\
\text { computationally feasible }\end{array}$ \\
\hline
\end{tabular}




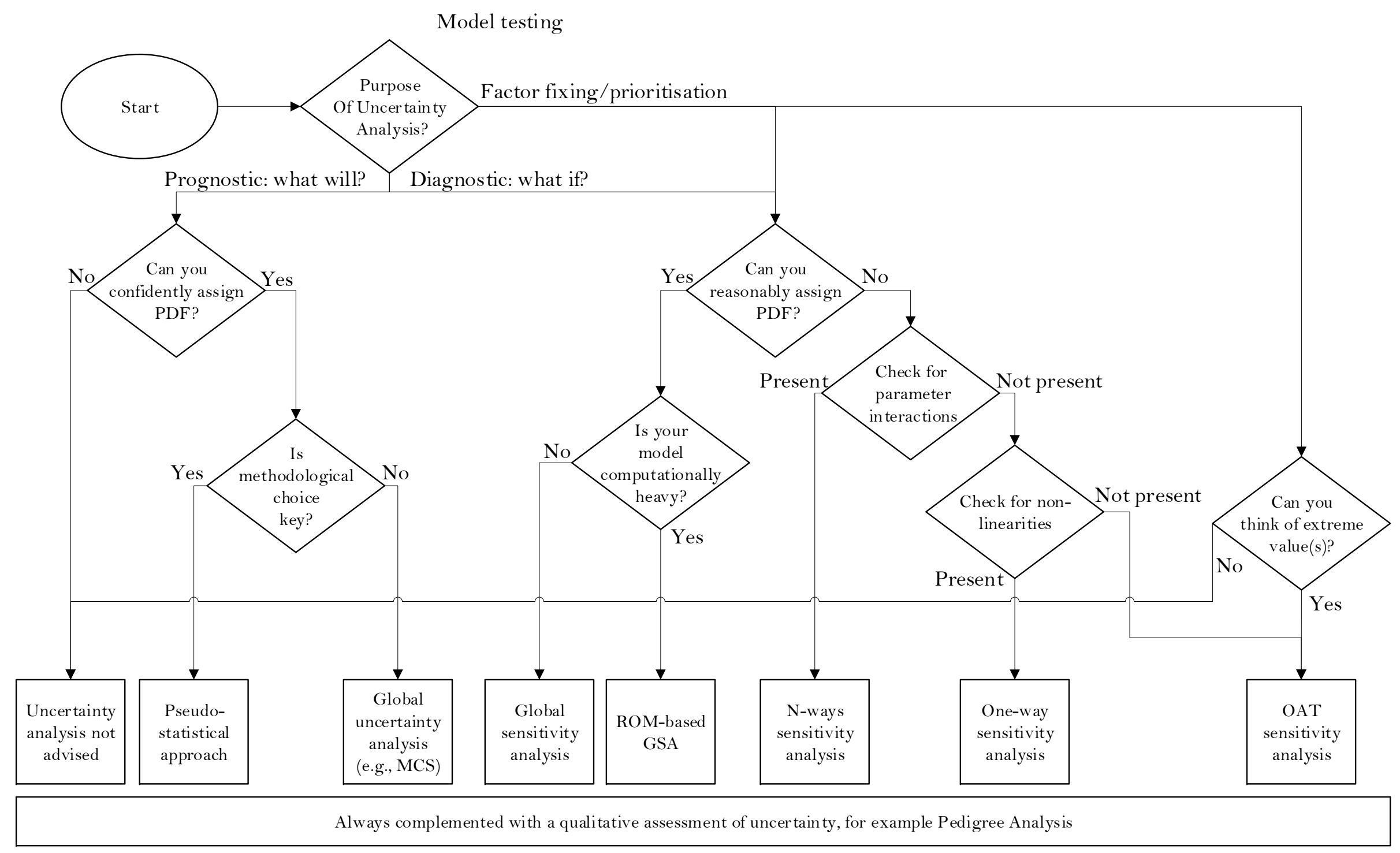

Figure 3-8. Scheme for initial uncertainty analysis selection guidance. 


\subsection{Advanced uses of uncertainty analysis in CCS TEA}

The previous sections discussed guidelines to select and apply methods for sound uncertainty analysis. These methods not only aid the critical analysis of the techno-economic potential of a CCS technology but also points towards specific areas that are candidates for further investigation. There are also, what can be called, advanced uses of uncertainty analysis, for instance in multiscale modelling for material and system design, design of experimental campaigns in pilot plants that are very expensive, design of CCS supply chains, CCS risk and safety analysis, and use of existing infrastructure in CCS value chain. Two such examples will be discussed here.

\subsubsection{Using uncertainty analysis for design of experiments}

Bayesian inference is the theoretical foundation upon which intelligent experimental design can be leveraged to inform the models used to characterize a process [195]. When model precision is considered unacceptably low for a process that needs to be further understood, measures should be undertaken to improve understanding of the process [196]. The technique of Bayesian inference, coupled with collection of experimental data, provides a means for reduction of model uncertainty, and thus refinement in understanding for a process systems application of interest. Sequential Design of Experiments (SDoE) is a framework that incorporates uncertainty-based criteria for selection of operating conditions for data collection and the use of the data for refining a stochastic process model in a cyclical manner. The SDoE procedure was previously summarized in Soepyan et al. [197], and demonstrated at pilot scale for a solvent-based $\mathrm{CO}_{2}$ capture system in Morgan et al. [198]. The SDoE process is represented schematically in Figure 3-9.

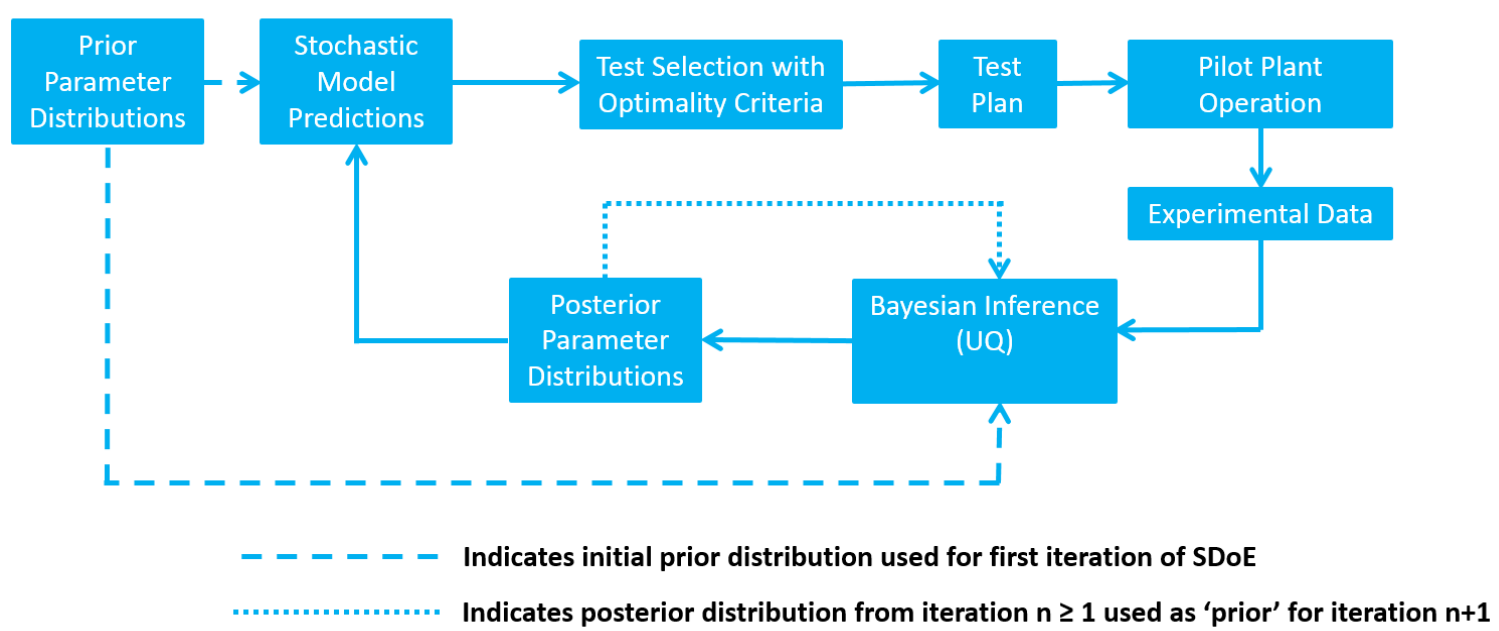

Figure 3-9. Schematic of Bayesian SDoE implemented for pilot plant campaign.

The SDoE methodology requires an initial process model, or a reduced-order surrogate model of the process, in which some of the parameters are characterized by probability density functions (PDFs), representing the parametric uncertainty. For the example of a solvent-based carbon capture system, uncertain parameters may include those related to the physical properties the system, reaction kinetics, and mass transfer and hydraulic models for the packing used in absorption and stripping columns [188-190]. Accurate characterization of mass transfer, interfacial area, and hydraulics, with quantified uncertainty, is necessary for representing the rate-based column models with a specific packing type. For chemical solvent systems, including the 
traditional amine-based systems, characterization of reaction kinetics is also essential. Physical properties such as viscosity, density, and surface tension are independent of packing type, although their uncertainties propagate through the mass transfer and interfacial area models, which are dependent upon the property models. However, previous work on physical properties uncertainty quantification for the MEA system for the aqueous monoethanolamine (MEA) system [188], Morgan et al. [189] has demonstrated that the most influential source of property model uncertainty is the thermodynamic framework. Accuracy in the thermodynamic framework is necessary for characterising the vapor-liquid equilibrium in column packing, heat of absorption, heat capacity, and reaction equilibrium constants for chemical systems.

The prior distributions on these uncertain parameters represent initial beliefs about the parameters before collection of data. At various process conditions covering the full operating space of interest, the process model is evaluated stochastically by sampling from the prior distribution of the parameters and calculating the model output for each point in the sample. At each process condition, the model output calculated from all samples may be used to estimate statistics (e.g. mean, variance, prediction intervals) for the model output. For the solvent-based $\mathrm{CO}_{2}$ capture system example, the operating space of interest represents feasible combinations of input variables including but not limited to solvent flowrate, flue gas flowrate, $\mathrm{CO}_{2}$ loading in solvent, and $\mathrm{CO}_{2}$ concentration in the flue gas. Some output variables of interest may include $\mathrm{CO}_{2}$ capture efficiency in the absorber and reboiler duty requirement in the stripper [191].

Along with the model prediction of uncertainty as a function of the process operating conditions, some optimality criteria [199], which are chosen based on specific experimental goals, are used to select a subset of the candidate set to be included in a test plan for data collection. For example, If a goal of a test campaign is to refine a model by reducing the parametric uncertainty, it may be desirable to collect data in operation conditions where the initial predicted uncertainty is relatively high. Experimental data are collected according to the test plan, and Bayesian inference is used to estimate posterior distributions of the parameters, which represent refined distributions of the parameters conditioned on the data observations. The model prediction of uncertainty is updated (generally reduced) as a result of refinement in the parameter estimates, and this information is used to determine a new test plan for further data collection. This results in the sequential nature of the SDoE process, in which the model is refined over multiple iterations. The decisions regarding the amount of data to collect in each iteration and the criteria for terminating the SDoE loop are left to the experimenter. In practice, however, these decisions may be dictated by limitations in time and money available for pilot-scale test campaigns.

A recent example [200] of SDoE execution for a large pilot-scale test campaign at Norway's Technology Centre Mongstad (TCM) is summarized in the following. In the example here, a Bayesian DoE was applied to an MEA baseline test campaign at Technology Centre Mongstad (TCM). Solvent flowrate, gas flowrate, and lean loading were variables in the test plan, serving as inputs to pre-existing fundamental models which are targeted for refinement using the experimental results. The objective of this experimental campaign was to minimize the maximum uncertainty in the operational space under consideration.

Figure 3-10 below illustrates the results of one DoE iteration for parametric refinement, which results in the following computationally projected improvement in the $95 \%$ confidence interval width of the capture percentage projection. Parameters targeted for refinement in this design of experiments related to, among other things, mass transfer characterization which directly affects 
equipment size and thus cost. Therefore, improving our understanding of these parameters also improves cost projections.

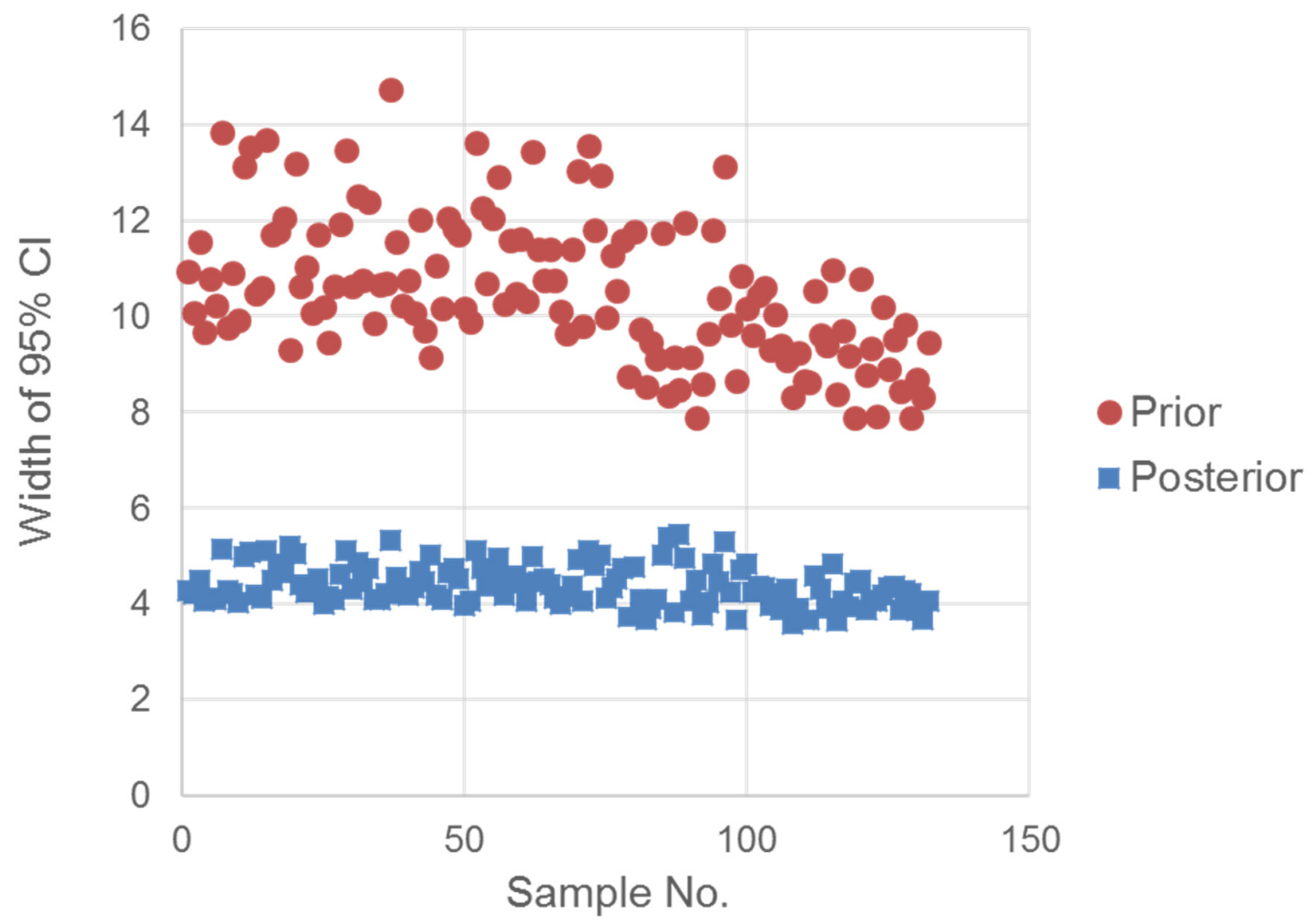

Figure 3-10. Example of results from Bayesian SDoE implemented for an MEA pilot plant campaign at Technology Centre Mongstad (TCM). The reduction in uncertainty (i.e. width of the $95 \%$ confidence interval) before and after the campaign is clearly visible.

\subsubsection{Design of CCS supply chains under uncertainty}

Even though uncertainties can be reduced through technology testing, uncertainties remain an inherent and important element of novel systems with limited large-scale industrial experience and must be taken into account to enable the design of cost-efficient energy systems [8]. Advanced uncertainty quantification approaches, often referred to as 'design under uncertainty' in the engineering field $[201,202]$, have been developed to account for uncertainty already during the design step to achieve better and more robust designs. In practise, an optimisation layer is added to an uncertainty propagation approach, like Monte Carlo simulation, to optimise system variables according to one or several targeted objective(s) related to the output distribution of key performance indicators (KPIs) as shown in Figure 3-11. The targeted objective(s) can be of different nature. One may want to minimize or maximize the mean value of the probability distribution of a given key performance indicator (KPI). Alternatively, one may aim to minimize the uncertainty range of a KPI distribution or to limit extreme values to reduce associated risks.

While design under uncertainty is being more and more considered in engineering approaches [202-204], only few studies have considered such approaches for design of CCS chains or its components. Cerillo-Briones and Ricardez-Sandoval [205] investigated the robust design of an absorber column under process uncertainties such as flue gas flow and temperature, as well as 
solvent characteristics. Bjerketvedt et al. [206], investigated optimal ship-based $\mathrm{CO}_{2}$ transport considering uncertainties in sailing time due to weather conditions, seasonal variations, future fuel cost, and risk of ship breakdown. Similarly, Knoope et al. [207], investigated the impact of price uncertainties on the decision to differentiate or expand investment in a $\mathrm{CO}_{2}$ infrastructure network using real option analysis. Meanwhile, Roussanaly et al. [208] performed an extensive study on the impact of technical, economic, and system uncertainties on the cost and design of CCS from a waste-to-energy plant.

In the work by Roussanaly et al. [208], one of the considered cases investigated the impact of uncertainties on the design and cost of a CCS chain based on solvent $\mathrm{CO}_{2}$ capture and a transport and storage infrastructure shared with nearby industries. An optimal design of this chain was developed considering two uncertainty scenarios: 1) "internal" uncertainties such as uncertainties in investment costs, steam, and electricity consumptions; and 2) internal uncertainties combined with uncertainties in the amount of $\mathrm{CO}_{2}$ captured from the other nearby industries (referred as "external" uncertainties). The evaluation showed that a more robust design could be achieved in the second scenario by optimising the design under uncertainties. A larger pipeline diameter is optimal in the second scenario although it results in a $\mathrm{CO}_{2}$ avoidance cost $5 € / \mathrm{tCO}_{2}$ avoided higher than in the first uncertainty scenario. Indeed, the optimal design of the first uncertainty scenario has a probability of $35 \%$ that it cannot accommodate all the $\mathrm{CO}_{2}$ captured from nearby industries. One of the main drawbacks of design under uncertainty is that it requires significantly more computational time than deterministic approaches, due to the combination of extensive Monte Carlo simulations and the optimisation of multiple variables. Furthermore, a robust modelling of the considered system, as well as a good understanding of the potential underlying uncertainties are required if meaningful results are to be achieved. Despite these challenges, design under uncertainty has shown to provide significant advantages compared to deterministic approaches: enabling cheaper designs or designs that can foster a higher value creation, enabling more robust designs, or limiting risks associated with expected uncertainties. It is therefore important to note that while design under uncertainty modelling approaches can be self-developed, several academic and commercial tools such as UQ lab and Oracle Crystal ball also offer this functionality (see also section 3.5). 


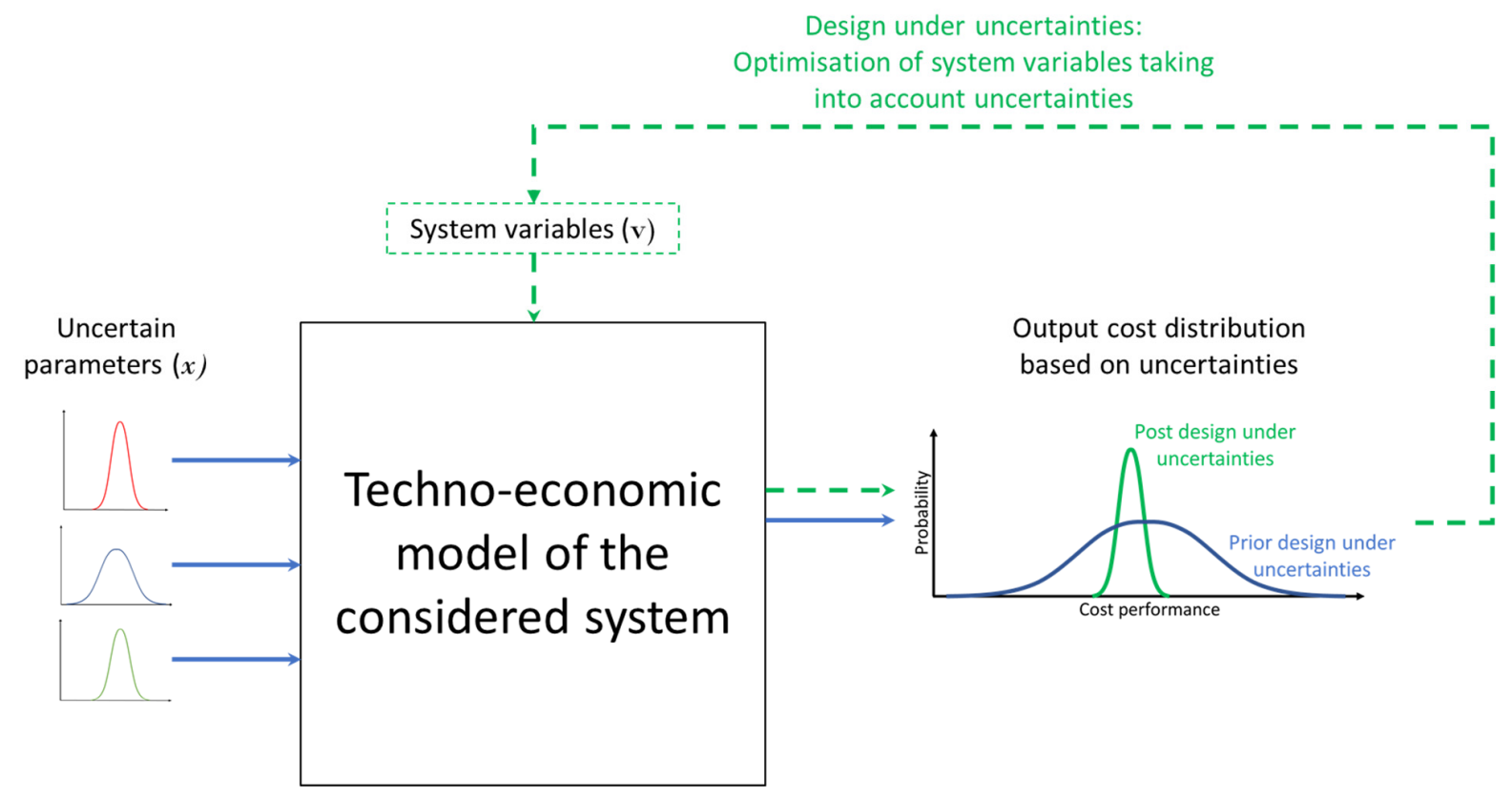

Figure 3-11. Schematic representation of design under uncertainties. The "classical" Monte Carlo simulation process is shown in blue while the additional design element is illustrated in green.

\subsection{Available software for uncertainty and sensitivity analysis}

With recent interest in uncertainty analysis from many researchers, engineers, and academic institutions, various software packages have been developed to perform uncertainty analysis in a structured manner. The aim of these tools is to provide more accessible, easy, and ready to use software and to facilitate the use of uncertainty analysis to a wider audience. However, selecting a suitable analysis tool for a specific application is not always straightforward: it requires to understand the options' relative merits, features, and performance level. We here try to provide a brief review of the current uncertainty analysis tools can be employed in (CCS) TEAs.

Commercially available tools for uncertainty propagation and sensitivity analysis include Crystal Ball, Pallisade's @RISK [209] and RISK AMP [210]. These three tools are Excel add-in's, which makes them easy to use also to practitioners less versed in programming languages. According to a review by Sugiyama [209], Crystal Ball is the easiest to use due to an excellent user guide and reference manuals, and the provision of a number of illustrative models [211]. All three packages include local and global sensitivity analysis methodologies using post-processing of the Monte Carlo simulations. A limitation of using excel based software for CCS TEA's, however, could be the integration with external software such as MATLAB, Python, or Aspen, which are often used for the process modelling of CCS technologies (this may especially be a limitation for starting TEA practitioners). It should be noted, however, that Aspen includes the capability to perform local SA for process modelling and techno economics, in addition it can be controlled from Excel using the Aspen Simulation Workbook add-in, which then allows the possibility to use these excel based UQ software packages.

In addition to the Excel-based packages, there is a number of recently established tools that have advanced capabilities in performing probabilistic uncertainty analysis. They include, e.g., surrogate-modelling methods such as Polynomial Chaos Expansion (PCE) or Bayesian inference 
with the Markov Chain Monte Carlo (MCMC) method ${ }^{24}$. They also include the calculation of local and global sensitivity indicators such as Perturbation, Morris, and Sobol indices and facilitate design optimisation under uncertainty. As a starting point, we name some here that are widely used: the Dakota toolkit is a $\mathrm{C} / \mathrm{C}++$ based tool developed by Sandia National Laboratories [212]; UQLab is a MATLAB based framework developed by the Chair of Risk, Safety and Uncertainty Quantification of ETH Zürich [213]; and FOQUS (the Framework for Optimization and Quantification of Uncertainty and Sensitivity), developed by the United States Department of Energy's Carbon Capture Simulation [214], is a Python-based framework that connects to several flowsheeting software using a graphic user interface the United States Department of Energy's Carbon Capture Simulation [192].

Among the discussed software above, the Dakota toolkit provides the most extensive range of UQ methodologies. It has more functionalities than the other two, including for instance methods for quantifying epistemic kinds of uncertainties. It is, however, easy to get lost in all the options it provides and may not be the best software for starting TEA practitioners. The fact that it is written in $\mathrm{C}++$ may also make it difficult to integrate with TEAs. Although all these tools provide extensive manuals and documentation, their use can seem quite daunting, especially if one does not have an advanced programming knowledge. In general, the choice of tool can be based on the programming language preference and most of the tools provide the advantage of being opensource type which allows new methods to be added and integrated. For this reason, it is recommended to start with a tool that provides the best technical guidance, e.g., through the inclusion of interactive procedures or step-by-step checklists. In addition, it is recommended to choose a tool that offers active discussion forums among the user community to help the user with methodological or model implementation issues. Examples include UQLab and FOQUS. Further guidance on the selection of uncertainty analysis tools can be sought for instance through online databases like swMATH. Table 3-8 summarizes the above considerations.

Table 3-8. Comparison of available software packages for uncertainty evaluation of (but not specific to) CCS techno-economic models.

\begin{tabular}{|l|c|c|c|c|c|c|}
\hline Software name & Crystal Ball & Palisade & Risk AMP & Dakota & UQLab & FOQUS \\
\hline Type & Commercial & Commercial & Commercial & Open access & Open access & Open access \\
\hline $\begin{array}{l}\text { OAT, One-way and N-ways } \\
\text { sensitivity analysis }\end{array}$ & $\checkmark$ & $\checkmark$ & $\checkmark$ & $\checkmark$ & $\checkmark$ & $\checkmark$ \\
\hline $\begin{array}{l}\text { Global uncertainty and sensitivity } \\
\text { analysis (Monte Carlo) }\end{array}$ & $\checkmark$ & $\checkmark$ & $\checkmark$ & $\checkmark$ & $\checkmark$ & $\checkmark$ \\
\hline $\begin{array}{l}\text { Reduced order global uncertainty } \\
\text { and sensitivity analysis }\end{array}$ & X & X & X & $\checkmark$ & $\checkmark$ & $\checkmark$ \\
\hline $\begin{array}{l}\text { Direct linking with external } \\
\text { software (e.g. MATLAB, Python) }\end{array}$ & X & X & X & $\checkmark$ & $\checkmark$ & $\checkmark$ \\
\hline $\begin{array}{l}\text { Linking with Aspen via Excel } \\
\text { interface }\end{array}$ & $\checkmark$ & $\checkmark$ & $\checkmark$ & $\checkmark$ & $\checkmark$ & $\checkmark$ \\
\hline $\begin{array}{l}\text { Availability of supporting } \\
\text { documentation/manual/training } \\
\text { materials }\end{array}$ & $\checkmark$ & $\checkmark$ & $\checkmark$ & $\checkmark$ & $\checkmark$ & $\checkmark$ \\
\hline $\begin{array}{l}\text { Level of programming skills } \\
\text { required }\end{array}$ & None & None & None & Intermediate & Intermediate & Intermediate \\
\hline
\end{tabular}

\footnotetext{
${ }^{24} \mathrm{~A}$ sampling technique to identify the posterior probability distribution of a parameter once the prior probability distribution has been determined.
} 


\subsection{Conclusions and recommendations}

Proper use of uncertainty analysis in the performance of CCS TEAs can provide more robust understanding of technical and cost performance to modelling practitioners as well as policy and decision-makers. While there is a growing appreciation over the importance of uncertainty evaluation to both development of models and reporting of results, it remains the case that they are not always evaluated and, when they are, they are often not evaluated using the most appropriate methods. This chapter provided a critical review of a selection of existing and emerging uncertainty analysis methods and provided guidelines on their use. It discussed good practices as well as pitfalls, provided guidance on how to select and use methods, and pointed to sources of further information when outside the scope of this work. It aspires to be a starting point for an audience getting acquainted with CCS TEA, or that wishes to improve their (knowledge of) TEAs.

The review showed that many different methods and approaches to uncertainty analysis exist. We emphasised that key to starting any uncertainty analysis is to first thoroughly define its purpose, and then to ensure that the most suitable type of uncertainty analysis for that purpose is selected (also in relation to the choice of techno-economic model itself). In addition, a good understanding of the methods' strengths and limitations is imperative. Finally, the choice also depends on the existing knowledge of the investigated technology and the associated TEA model and its inputs (including potential parameter ranges - and possibly probability distribution).

The simplest method for model diagnostics ("what If" questions), and one that should as a minimum be applied (in the first instance), is one-at-a-time sensitivity analysis, but we recommend one-way or N-ways sensitivity analysis since most TEAs include non-linearities and parameter interactions. OAT has a really very limited use (although it is actually the most used), except in the case of model testing to extreme inputs, and also then it is arguably better to test the model to extreme scenarios (i.e., combinations of extreme inputs, NAT sensitivity analysis). To address prognostic ("what will") questions, probabilistic methods are most appropriate, but caution must be applied in their use as the distribution of results is only as good as the distribution of inputs. If distributions cannot be quantified with good confidence, then probabilistic methods are simply not suitable to answer prognostic questions (but may still be suitable to answer diagnostic questions). Finally, we highly recommend always complementing quantitative uncertainty analysis with qualitative methods, because they provide insights into the kinds of uncertainty that are unquantifiable, especially relevant to policy and decision making.

In addition to the classic uses of uncertainty analysis, advanced uses can come to the advantage of TEA practitioners and CCS developers. We exemplified that iterative uncertainty analysis can aid the design of experimental campaigns by pinpointing regions that need further investigation, allowing the experiments to be more efficient and less costly. Another advanced use is in the development of CCS infrastructure, where integrating design under uncertainty at the planning stage can enable more robust systems.

The (un)availability of suitable software is something that has hampered (and continues to hamper) the use of more advanced uncertainty analysis in CCS TEA. Although possible, it may be difficult for starting practitioners to combine Excel-based (and very user-friendly) uncertainty analysis addins with process simulation software like Aspen (although Aspen does allow local sensitivity analysis on techno-economics within its suite). Further expanding the capabilities of process simulation software to include advanced global uncertainty approaches would be very helpful (the 
gProms software has already included this option). For some flowsheeting software, TEA practitioners can link their simulations to existing (and quite elaborate) uncertainty quantification tools in MATLAB or other programming languages, but this requires substantially more knowledge of, and skill in, programming and the software layers underlying process simulation software to make the connection. Easier ways to connect different software would be highly desirable. Also, further improvement of the user-friendliness of existing UQ toolboxes (e.g., by including graphic user interfaces) would aid in the further adoption of advanced uncertainty analysis methods. Finally, a key challenge is to fully combine detailed technical and cost models, to allow for integrated (instead of piecewise) TEA uncertainty analysis.

CCS remains a technology that has had so far limited large-scale implementation and therefore inherently large uncertainties. Uncertainty analysis has, thus, an important role to play in TEA of CCS technologies and systems and there are many opportunities to bring our use of uncertainty analysis to a level higher than currently often done. Hopefully, this work inspires the use of the available possibilities and the continued development towards robust and meaningful CCS TEAs. 


\section{Appendix A: Screening questions for FOAK cost estimates}

Table A-1. Initial screening questions for design and cost estimates for advanced (pre-commercial) technologies (Based on [47])

\begin{tabular}{|c|c|c|}
\hline No. & Screening question for pre-commercial technologies & How does this affect the FOAK cost estimate? \\
\hline 1 & $\begin{array}{l}\text { Is the patent and/or intellectual property holder for the technology } \\
\text { and/or process involved in this cost estimate? }\end{array}$ & $\begin{array}{l}\text { Without the cooperation of this entity, there can be significant gaps in the cost and design basis } \\
\text { estimates. }\end{array}$ \\
\hline 2 & How long has this technology been in development? & $\begin{array}{l}\text { The longer the technology has been under development and the more pilot-scale and/or bench- } \\
\text { scale operating data that are available, the higher the confidence in the overall cost estimate. }\end{array}$ \\
\hline 3 & $\begin{array}{l}\text { What is the intended scale of the technology relative to the size of } \\
\text { previous/current installations? (e.g., is the process being scaled up by } \\
10 \text { times? } 100 \text { times?) Are there "inflection points" where either the } \\
\text { design and/or materials needed for the process become more or less } \\
\text { expensive? }\end{array}$ & $\begin{array}{l}\text { The ratio of proposed to current scale of the technology factors heavily into the costing method } \\
\text { selected and its accuracy. E.g., a large ratio in a factored cost estimate is typically coupled with a } \\
\text { lower degree of accuracy for the final estimate. }\end{array}$ \\
\hline 4 & $\begin{array}{l}\text { What is the size of the closest analogue to this process and can it be } \\
\text { utilized as a design and/or cost estimating benchmark? }\end{array}$ & This heavily impacts the accuracy and applicability of a factored or weighted cost estimate. \\
\hline 5 & $\begin{array}{l}\text { How would you characterize the current level of maturity of the } \\
\text { technology? For example: } \\
\text { - Does it have a "proof of concept" prototype? } \\
\text { - Has it received Underwriter's Laboratory (UL) certification? } \\
\text { - Have investors been sought or secured for its development? } \\
\text { Is it owned by a parent company and/or governing entity? }\end{array}$ & $\begin{array}{l}\text { The level of maturity weighs heavily on the targeted level of cost estimate detail and the accuracy } \\
\text { bounds on the estimate. } \\
\text { For example, FOAK technology has a lower risk level and easier market entry if it is UL } \\
\text { certified. However, the cost for certification can range from hundreds of thousands to millions of } \\
\text { dollars, depending on technology complexity and testing process results. }\end{array}$ \\
\hline 6 & $\begin{array}{l}\text { What are the types and quantities of mass and energy inputs and } \\
\text { outputs from the facility? }\end{array}$ & $\begin{array}{l}\text { As with mature technologies, without a detailed list of inputs and outputs there is low fidelity in } \\
\text { cost estimates. }\end{array}$ \\
\hline 7 & $\begin{array}{l}\text { Does the pre-commercial technology produce any "hazardous" } \\
\text { products or outputs from the facility, based on applicable codes? } \\
\text { (Examples of hazardous materials that require mitigation measures } \\
\text { include: non-potable water contaminated with heavy metals; spent } \\
\text { catalyst materials; coal combustion bottom ash and fly ash with levels } \\
\text { of mercury, unburned carbon, cadmium, and other heavy metals that } \\
\text { exceed regulatory limits.) }\end{array}$ & $\begin{array}{l}\text { O\&M costs can be affected significantly by the presence of hazardous wastes. Representative } \\
\text { disposal cost is roughly twice that for non-hazardous solid waste (unit disposal rate of } \\
\text { ( } \$ 80.00 / \text { ton for hazardous materials vs. } \$ 38.00 / \text { ton for non-hazardous waste [43]. }\end{array}$ \\
\hline 8 & $\begin{array}{l}\text { What upstream and or downstream dependencies does the facility rely } \\
\text { on to be operational and economically viable? } \\
\text { A commercial power plant will likely have a dozen or more upstream } \\
\text { entities (fuel suppliers, reagent suppliers, equipment suppliers, } \\
\text { contractors, consultants, permitting agencies, etc.) and a similar number } \\
\text { of downstream entities (waste product off-takers, independent system } \\
\text { operator, discharge permitting agencies, etc.) that must be managed. }\end{array}$ & $\begin{array}{l}\text { The greater the number of upstream and downstream entities the greater the economic risk from } \\
\text { exposure to market forces and supplier requirements. } \\
\text { Compared to a mature commercial technology, a FOAK facility can be expected to have } \\
\text { significantly more equipment suppliers, contractors, consultants, and other personnel over an } \\
\text { extended period of time, which could increase the man-hour costs of the project schedule by } 2-3 \\
\text { times that of a commercial facility. }\end{array}$ \\
\hline
\end{tabular}




\section{Table A-1. (cont'd)}

Is the technology using a chemical reagent or other material that must be continuously or periodically supplied? If so, is it a widely available commercial product, a proprietary product, or a newly developed product?

Have the technical specifications for the process been distributed to suppliers or manufacturers for review? If so, do they have the capability and experience to supply the equipment? Are they willing to establish a Master Service Agreement to build the equipment?

How have the risks associated with design, construction and operation been incorporated into the process design and cost estimate? (see Section 1.4.2.2 for descriptions of risk analysis tools.) \begin{tabular}{l|l} 
What cost estimating disciplines apply to the facility (e.g., chemical, \\
civil, electrical, mechanical, and structural engineering; procurement
\end{tabular} specialists; construction management specialists)?

A significantly affect the estimated O\&M cost. For standard materials, the unit cost of ow-volume quantities could be 15-25 percent or more than high-volume quantities typical of a commercial coal power plant. Costs for proprietary or newly-developed products could be substantially higher with greater risk of supply availability.

Review by industry-aligned manufacturers will help support assumptions regarding potential technology suppliers. Indications that technology requirements are aligned with supplier experience base will improve the fidelity of the design basis and cost estimate.

Quantifying these risks is critical to establishing the appropriate direct, indirect, and contingency costs in the estimate. A high-fidelity estimate will require a detailed evaluation of these risks.

This influences several elements of the capital cost estimate and is especially important for a detailed (definitive) cost study of a specific project. Disciplines vary with technology and scope of what is included in the FOAK cost estimate. 


\section{Appendix B: Location cost factors and other contingency cost guidelines for large-scale energy projects}

Table B-1. Geographic location cost factors compared to the Netherlands [37]

\begin{tabular}{|l|c|c|c|c|}
\hline Location & $\begin{array}{l}\text { Materials } \\
\text { Cost Factor }\end{array}$ & $\begin{array}{l}\text { Labour } \\
\text { Productivity } \\
\text { Factor }\end{array}$ & $\begin{array}{l}\text { Labour } \\
\text { Cost Factor }\end{array}$ & $\begin{array}{l}\text { Added CCS } \\
\text { Contingency } \\
\text { factor* }\end{array}$ \\
\hline The Netherlands & 1.00 & 1.00 & 1.00 & $10 \%$ \\
\hline Eastern Europe & 0.92 & 1.28 & 0.40 & $10 \%$ \\
\hline USA (mid-west) & 0.94 & 0.95 & 0.92 & $10 \%$ \\
\hline Canada & 1.07 & 1.12 & 0.96 & $10 \%$ \\
\hline South Africa & 1.03 & 2.24 & 0.70 & $10 \%$ \\
\hline Australia & 1.00 & 1.23 & 1.38 & $10 \%$ \\
\hline India & 0.93 & 2.42 & 0.26 & $15 \%$ \\
\hline China & 0.77 & 2.29 & 0.16 & $15 \%$ \\
\hline Japan & 0.91 & 0.98 & 0.68 & $10 \%$ \\
\hline South-East Asia & 0.92 & 1.78 & 0.24 & $15 \%$ \\
\hline South America & 0.97 & 1.54 & 0.28 & $20 \%$ \\
\hline Middle East & 0.91 & 1.84 & 0.24 & $20 \%$ \\
\hline
\end{tabular}

*This factor refers to additional potential execution risks at a particular location for the implementation of a large CCS project; it must not be confused with project and process contingency costs, which are described in Section 1.3.2 of this report. 
Table B-2. Other contingency cost guidelines dependent on level of technology maturity and phase of project development (data from $[40,215]$ )

\begin{tabular}{|c|c|c|c|c|c|}
\hline \multirow{2}{*}{$\begin{array}{l}\text { Level of Project } \\
\text { Development }\end{array}$} & \multicolumn{5}{|c|}{ Phase of Project Development ${ }^{b}$} \\
\hline & $\begin{array}{l}\text { Concept } \\
\text { study }\end{array}$ & $\begin{array}{l}\text { Scoping } \\
\text { study }\end{array}$ & $\begin{array}{c}\text { Pre- } \\
\text { feasibility }\end{array}$ & $\begin{array}{l}\text { Feasibility } \\
\text { study }\end{array}$ & $\begin{array}{l}\text { Engineering } \\
\text { completed }\end{array}$ \\
\hline First-of-a-kind project & \multicolumn{5}{|c|}{ (All figures are percentages of the project overnight cost) } \\
\hline Process contingency & 50 & 35 & 20 & 15 & 10 \\
\hline Project contingency & 50 & 35 & 20 & 15 & 10 \\
\hline Total contingencies & 100 & 70 & 40 & 30 & 20 \\
\hline Supplementary funds & 50 & 40 & 30 & 20 & 10 \\
\hline \multicolumn{6}{|l|}{ Early-mover project } \\
\hline Process contingency & 25 & 15 & 10 & 5 & 5 \\
\hline Project contingency & 40 & 30 & 20 & 15 & 10 \\
\hline Total contingencies & 65 & 45 & 30 & 20 & 15 \\
\hline Supplementary funds & 30 & 20 & 15 & 10 & 5 \\
\hline \multicolumn{6}{|l|}{$\mathbf{N}^{\text {th }}$-of-a-kind project } \\
\hline Process contingency & 0 & 0 & 0 & 0 & 0 \\
\hline Project contingency & 30 & 25 & 20 & 10 & 5 \\
\hline Total contingencies & 30 & 25 & 20 & 10 & 5 \\
\hline Supplementary funds & 25 & 15 & 10 & 5 & 2.5 \\
\hline
\end{tabular}

a Project level definitions: First-of-a-Kind means $<10$ commercial-scale demonstrations worldwide or first demonstration in the project region; Early Mover means $>10$ and $<20$ commercial-scale demonstrations worldwide or $<3$ demonstrations in the project region; $\mathrm{N}^{\text {th }}$-of-a-Kind means $>20$ demonstrations worldwide and $>3$ in the project region (Greig, et al., 2014).

b Project Phase definitions: Scoping Study $=\sim 1 \%$ of engineering effort w/ accuracy of $-20 /+50 \%$; Pre-feasibility Study $=\sim 10 \%$ of engineering effort $\mathrm{w} /$ accuracy of $-15 /+30 \%$; Feasibility Study $=>40 \%$ of engineering effort $\mathrm{w} /$ accuracy of $-10 /+20 \%$. No similar definitions for Concept Study and Engineering Completed [40].

These guidelines were based in part on a review of cost estimates at different stages of ten recent large-scale energy projects whose cost escalations are shown below in Figure B-1. Further details are available in Greig, et al. [40]. 


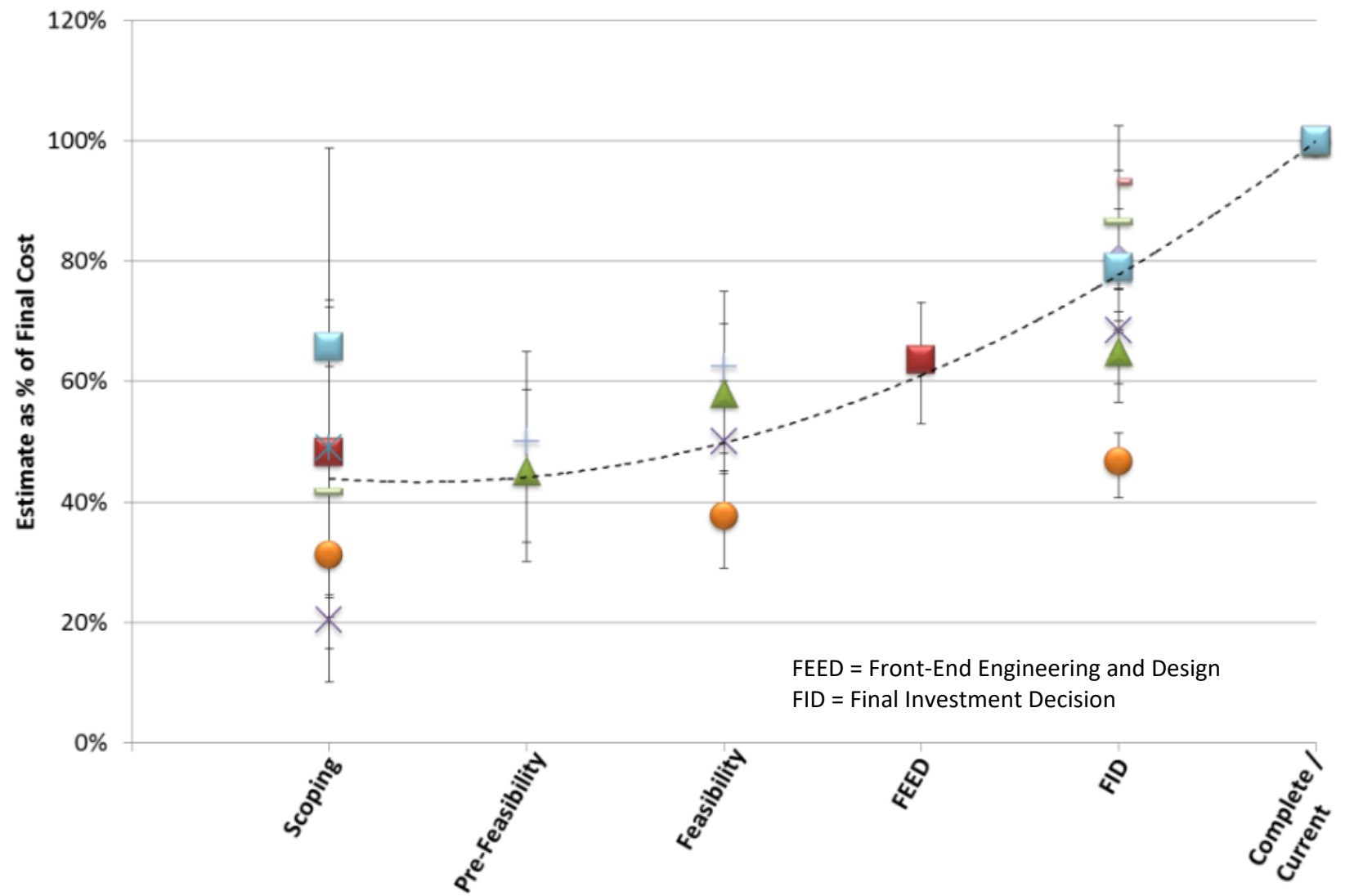

Figure B-1. Illustration of project estimate escalation through the phases of ten greenfield projects untaken during the ten years preceding this study [40] 


\section{Appendix C: Example assumptions and results for cost estimates using experience curves}

To illustrate the use of experience curves for NOAK cost projections, this appendix shows examples of the assumptions and results reported by IEAGHG [56] for four types of power plants (PC, NGCC, IGCC, and oxyfuel) equipped with carbon capture systems. In that study, the Integrated Environmental Control Model (IECM) was first used to estimate the current cost of each plant on a consistent basis for a U.S. facility with a net power output of approximately $500 \mathrm{MW}$, a levelised capacity factor of $75 \%$, and a carbon capture system removing $90 \%$ of the $\mathrm{CO}_{2}$ and compressing it to $13.8 \mathrm{MPa}$. All coal-based plants used a bituminous coal with $2.1 \%$ sulphur. All costs were in constant 2002 US dollars) and exclude the costs of $\mathrm{CO}_{2}$ transport and storage.

These estimates of initial cost were then used to project future costs using technology-specific learning rates, following the methodology outlined in Section 1.5 of this report. Results of an uncertainty analysis also are illustrated in this appendix.

Table C-1. Plant decomposition and sub-section cost allocations for four fossil fuel power plants with carbon capture systems [56]

\begin{tabular}{|c|c|c|c|c|c|c|}
\hline \multirow{3}{*}{ Plant Type \& Technology } & \multicolumn{6}{|c|}{ Total Plant Costs (\$2002) } \\
\hline & \multicolumn{2}{|c|}{ Capital Cost } & \multicolumn{2}{|c|}{ Total O\&M Cost ${ }^{5}$} & \multicolumn{2}{|c|}{ Total $\mathrm{COE}^{6,7}$} \\
\hline & $\$ / \mathrm{kW}$ & $\%$ Total & $\$ / \mathrm{MWh}$ & $\%$ Total & $\$ / \overline{M W h}$ & $\%$ Total \\
\hline NGCC Plant $^{1}$ & 916 & 100 & 38.5 & 100 & 59.1 & 100 \\
\hline GTCC (power block) & 660 & 72 & 2.2 & 6 & 17.1 & 29 \\
\hline $\mathrm{CO}_{2}$ capture (amine system) & 218 & 24 & 2.4 & 6 & 7.3 & 12 \\
\hline $\mathrm{CO}_{2}$ compression & 38 & 4 & 0.2 & 0 & 1.0 & 2 \\
\hline Fuel cost & 0 & 0 & 33.6 & 87 & 33.6 & 57 \\
\hline PC Plant $^{2}$ & 1,962 & 100 & 29.3 & 100 & 73.4 & 100 \\
\hline PC Boiler/turbine-generator area & 1,282 & 65 & 5.7 & 19 & 34.5 & 47 \\
\hline AP controls (SCR, ESP, FGD) & 241 & 12 & 4.1 & 14 & 9.5 & 13 \\
\hline $\mathrm{CO}_{2}$ capture (amine system) & 353 & 18 & 7.2 & 25 & 15.2 & 21 \\
\hline $\mathrm{CO}_{2}$ compression & 86 & 4 & 0.4 & 1 & 2.3 & 3 \\
\hline Fuel cost & 0 & 0 & 11.9 & 41 & 11.9 & 16 \\
\hline IGCC Plant ${ }^{3}$ & 1,831 & 100 & 21.3 & 100 & 62.6 & 100 \\
\hline Air separation unit & 323 & 18 & 1.7 & 8 & 8.9 & 14 \\
\hline Gasifier area & 494 & 27 & 3.7 & 17 & 14.8 & 24 \\
\hline Sulfur removal/recovery & 110 & 6 & 0.6 & 3 & 3.1 & 5 \\
\hline $\mathrm{CO}_{2}$ capture (WGS/Selexol) & 246 & 13 & 1.6 & 7 & 7.1 & 11 \\
\hline $\mathrm{CO}_{2}$ compression & 42 & 2 & 0.3 & 1 & 1.2 & 2 \\
\hline GTCC (power block) & 616 & 34 & 2.0 & 9 & 15.8 & 25 \\
\hline Fuel cost & 0 & 0 & 11.6 & 54 & 11.6 & 19 \\
\hline Oxyfuel Plant ${ }^{4}$ & 2,417 & 100 & 24.4 & 100 & 78.9 & 100 \\
\hline Air separation unit & 779 & 32 & 3.1 & 13 & 20.6 & 26 \\
\hline PC boiler/turbine generator area & 1,280 & 53 & 5.6 & 23 & 34.4 & 44 \\
\hline AP controls (ESP, FGD) & 132 & 5 & 2.7 & 11 & 5.7 & 7 \\
\hline $\mathrm{CO}_{2}$ distillation & 160 & 7 & 1.4 & 6 & 5.0 & 6 \\
\hline $\mathrm{CO}_{2}$ compression & 66 & 3 & 0.5 & 2 & 1.9 & 2 \\
\hline Fuel cost & 0 & 0 & 11.2 & 46 & 11.2 & 14 \\
\hline
\end{tabular}


Table C-2. Assumed learning rates for power plant sub-sections [56]

\begin{tabular}{|c|c|c|c|c|c|c|c|c|c|}
\hline \multirow{3}{*}{ Plant Type \& Technology } & \multirow{3}{*}{$\begin{array}{l}\text { Analogous } \\
\text { Technology }\end{array}$} & \multicolumn{8}{|c|}{ Learning Rates } \\
\hline & & \multicolumn{4}{|c|}{ Capital Costs } & \multicolumn{4}{|c|}{ O\&M Costs } \\
\hline & & Nominal & & an & & Nominal & & an & \\
\hline \multicolumn{10}{|l|}{ NGCC Plant } \\
\hline GTCC (power block) & GTCC & 0.10 & 0.05 & - & 0.15 & 0.06 & 0.00 & - & 0.10 \\
\hline $\mathrm{CO}_{2}$ capture (amine system) & FGD & 0.11 & 0.06 & - & 0.17 & 0.22 & 0.10 & - & 0.30 \\
\hline $\mathrm{CO}_{2}$ compression & (same) & 0.00 & 0.00 & - & 0.10 & 0.00 & 0.00 & - & 0.10 \\
\hline Fuel cost & & & & & & 0.04 & 0.00 & - & 0.05 \\
\hline \multicolumn{10}{|l|}{ PC Plant } \\
\hline PC Boiler/turbine-generator area & PC boiler & 0.06 & 0.03 & - & 0.09 & 0.15 & 0.07 & - & 0.30 \\
\hline AP controls (SCR, ESP, FGD) & FGD/SCR & 0.12 & 0.06 & - & 0.18 & 0.22 & 0.10 & - & 0.30 \\
\hline $\mathrm{CO}_{2}$ capture (amine system) & FGD & 0.11 & 0.06 & - & 0.17 & 0.22 & 0.10 & - & 0.30 \\
\hline $\mathrm{CO}_{2}$ compression & (same) & 0.00 & 0.00 & - & 0.10 & 0.00 & 0.00 & - & 0.10 \\
\hline Fuel cost & & & & & & 0.04 & 0.00 & - & 0.05 \\
\hline \multicolumn{10}{|l|}{ IGCC Plant } \\
\hline Air separation unit & $\mathrm{O}_{2}$ prod & 0.10 & 0.05 & - & 0.15 & 0.05 & 0.00 & - & 0.10 \\
\hline Gasifier area & LNG prod & 0.14 & 0.07 & - & 0.21 & 0.12 & 0.05 & - & 0.20 \\
\hline Sulfur removal/recovery & FGD & 0.11 & 0.06 & - & 0.17 & 0.22 & 0.10 & - & 0.30 \\
\hline $\mathrm{CO}_{2}$ capture (WGS/Selexol) & FGD/SCR & 0.12 & 0.06 & - & 0.18 & 0.22 & 0.10 & - & 0.30 \\
\hline $\mathrm{CO}_{2}$ compression & (same) & 0.00 & 0.00 & - & 0.10 & 0.00 & 0.00 & - & 0.10 \\
\hline GTCC (power block) & GTCC & 0.10 & 0.05 & - & 0.15 & 0.06 & 0.00 & - & 0.10 \\
\hline Fuel cost & & & & & & 0.04 & 0.00 & - & 0.05 \\
\hline \multicolumn{10}{|l|}{ Oxyfuel Plant } \\
\hline Air separation unit & $\mathrm{O}_{2}$ prod & 0.10 & 0.05 & - & 0.15 & 0.05 & 0.00 & - & 0.10 \\
\hline $\mathrm{PC}$ boiler/turbine generator area & PC boiler & 0.06 & 0.03 & - & 0.09 & 0.15 & 0.07 & - & 0.30 \\
\hline AP controls (ESP, FGD) & FGD & 0.11 & 0.06 & - & 0.17 & 0.22 & 0.10 & - & 0.30 \\
\hline $\mathrm{CO}_{2}$ distillation & LNG prod & 0.14 & 0.07 & - & 0.21 & 0.12 & 0.05 & - & 0.20 \\
\hline $\mathrm{CO}_{2}$ compression & (same) & 0.00 & 0.00 & - & 0.10 & 0.00 & 0.00 & - & 0.10 \\
\hline Fuel cost & & & & & & 0.04 & 0.00 & - & 0.05 \\
\hline
\end{tabular}


Table C-3. Estimated current capacity (MW-equivalent) of plant sub-sections [56]

\begin{tabular}{|c|c|}
\hline Plant Type \& Technology & $\begin{array}{c}\text { Current } \\
\text { Capacity } \\
\text { (MW) }\end{array}$ \\
\hline \multicolumn{2}{|l|}{ NGCC Plant } \\
\hline GTCC (power block) & 240,000 \\
\hline $\mathrm{CO}_{2}$ capture (amine system) & 10,000 \\
\hline $\mathrm{CO}_{2}$ compression & 10,000 \\
\hline \multicolumn{2}{|l|}{ PC Plant } \\
\hline PC Boiler/turbine-generator area & 120,000 \\
\hline AP controls (SCR, ESP, FGD) & 230,000 \\
\hline $\mathrm{CO}_{2}$ capture (amine system) & 10,000 \\
\hline $\mathrm{CO}_{2}$ compression & 10,000 \\
\hline \multicolumn{2}{|l|}{ IGCC Plant } \\
\hline Air separation unit & 50,000 \\
\hline Gasifier area & 10,000 \\
\hline Sulfur removal/recovery & 50,000 \\
\hline $\mathrm{CO}_{2}$ capture (WGS/selexol) & 10,000 \\
\hline $\mathrm{CO}_{2}$ compression & 10,000 \\
\hline GTCC (power block) & 240,000 \\
\hline \multicolumn{2}{|l|}{ Oxyfuel Plant } \\
\hline Air separation unit & 50,000 \\
\hline PC boiler/turbine generator area & 120,000 \\
\hline AP controls (ESP, FGD) & 230,000 \\
\hline $\mathrm{CO}_{2}$ distillation & 10,000 \\
\hline $\mathrm{CO}_{2}$ compression & 10,000 \\
\hline
\end{tabular}

Table C-4. Effect of key parameter values on cost projections for a PC plant with $\mathrm{CO}_{2}$ capture [56]

\begin{tabular}{|c|c|c|c|c|c|c|c|c|}
\hline \multirow[b]{2}{*}{ PC Sensitivity Case } & \multicolumn{4}{|c|}{ Capital Cost (\$/kW) } & \multicolumn{4}{|c|}{ COE (\$/MWh) } \\
\hline & $\begin{array}{l}\text { Learning } \\
\text { Rate }\end{array}$ & $\begin{array}{l}\text { Initial } \\
\text { Value }\end{array}$ & $\begin{array}{l}\text { Final } \\
\text { Value }\end{array}$ & $\%$ Change & $\begin{array}{l}\text { Learning } \\
\text { Rate }\end{array}$ & $\begin{array}{l}\text { Initial } \\
\text { Value }\end{array}$ & $\begin{array}{l}\text { Final } \\
\text { Value }\end{array}$ & $\%$ Change \\
\hline Nominal Base Case Assumptions & 0.021 & 1,962 & 1,783 & $9.1 \%$ & 0.035 & 73.4 & 62.8 & $14.4 \%$ \\
\hline Learning Starts with First Plant & 0.013 & 1,962 & 1,764 & $10.1 \%$ & 0.024 & 73.4 & 60.8 & $17.2 \%$ \\
\hline Learning up to $50 \mathrm{GW}$ & 0.018 & 1,962 & 1,846 & $5.9 \%$ & 0.031 & 73.4 & 66.0 & $10.1 \%$ \\
\hline Current Capture Capacity $=0 \mathrm{GW}$ & 0.026 & 1,962 & 1,744 & $11.1 \%$ & 0.042 & 73.4 & 60.9 & $17.1 \%$ \\
\hline Non-CSS Exp. Multipliers = 2.0 & 0.029 & 1,962 & 1,723 & $12.2 \%$ & 0.068 & 73.4 & 60.4 & $17.8 \%$ \\
\hline Coal Price $=\$ 1.5 / \mathrm{GJ}$ & 0.021 & 1,965 & 1,786 & $9.1 \%$ & 0.035 & 79.6 & 68.2 & $14.3 \%$ \\
\hline $\mathrm{FCF}=11 \%, \mathrm{CF}=85 \%$ & 0.021 & 1,963 & 1,785 & $9.1 \%$ & 0.039 & 57.2 & 48.2 & $15.7 \%$ \\
\hline
\end{tabular}




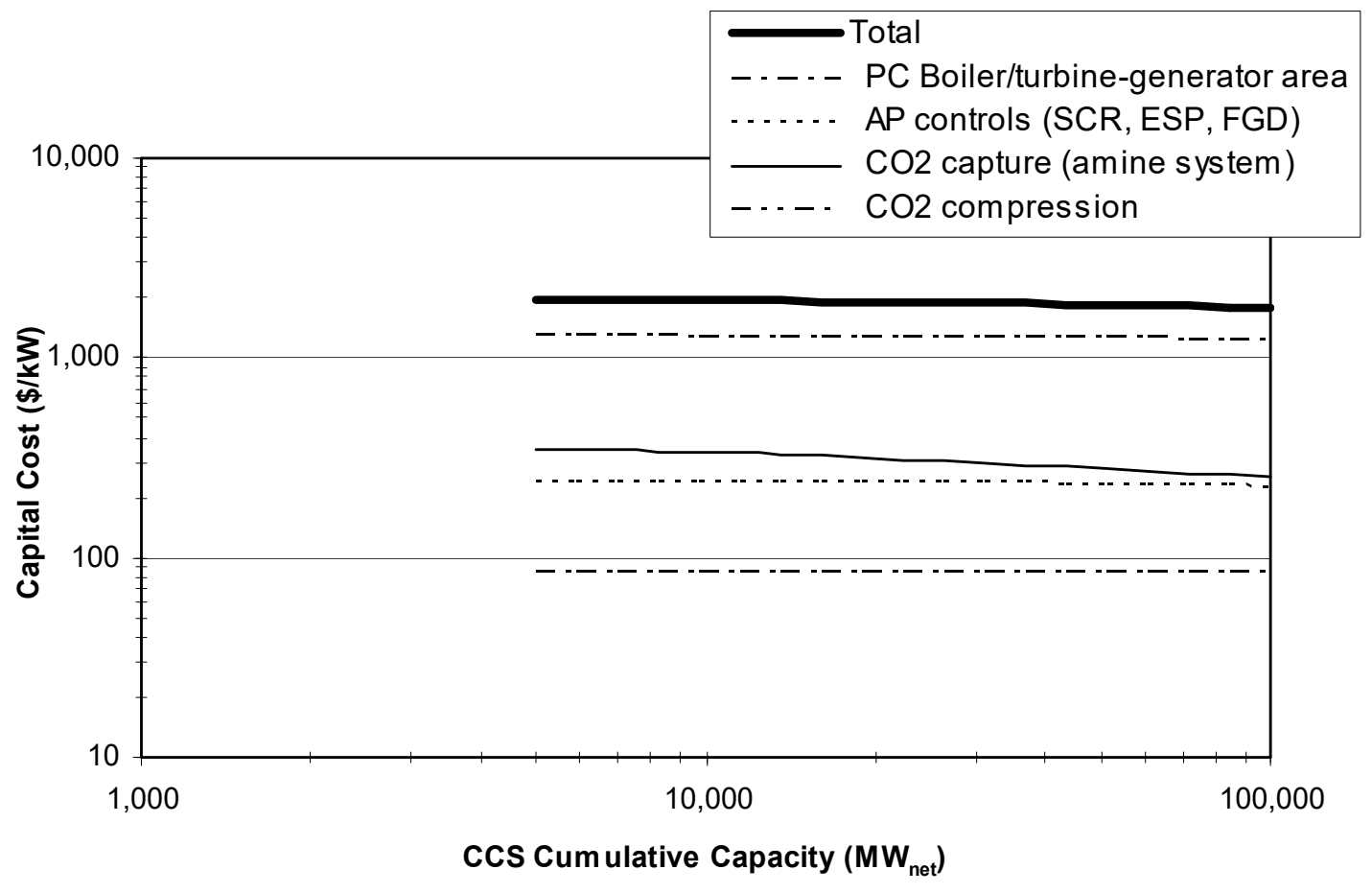

Figure C-1. Illustrative capital cost trends for components of a PC plant with $\mathrm{CO}_{2}$ capture [56]

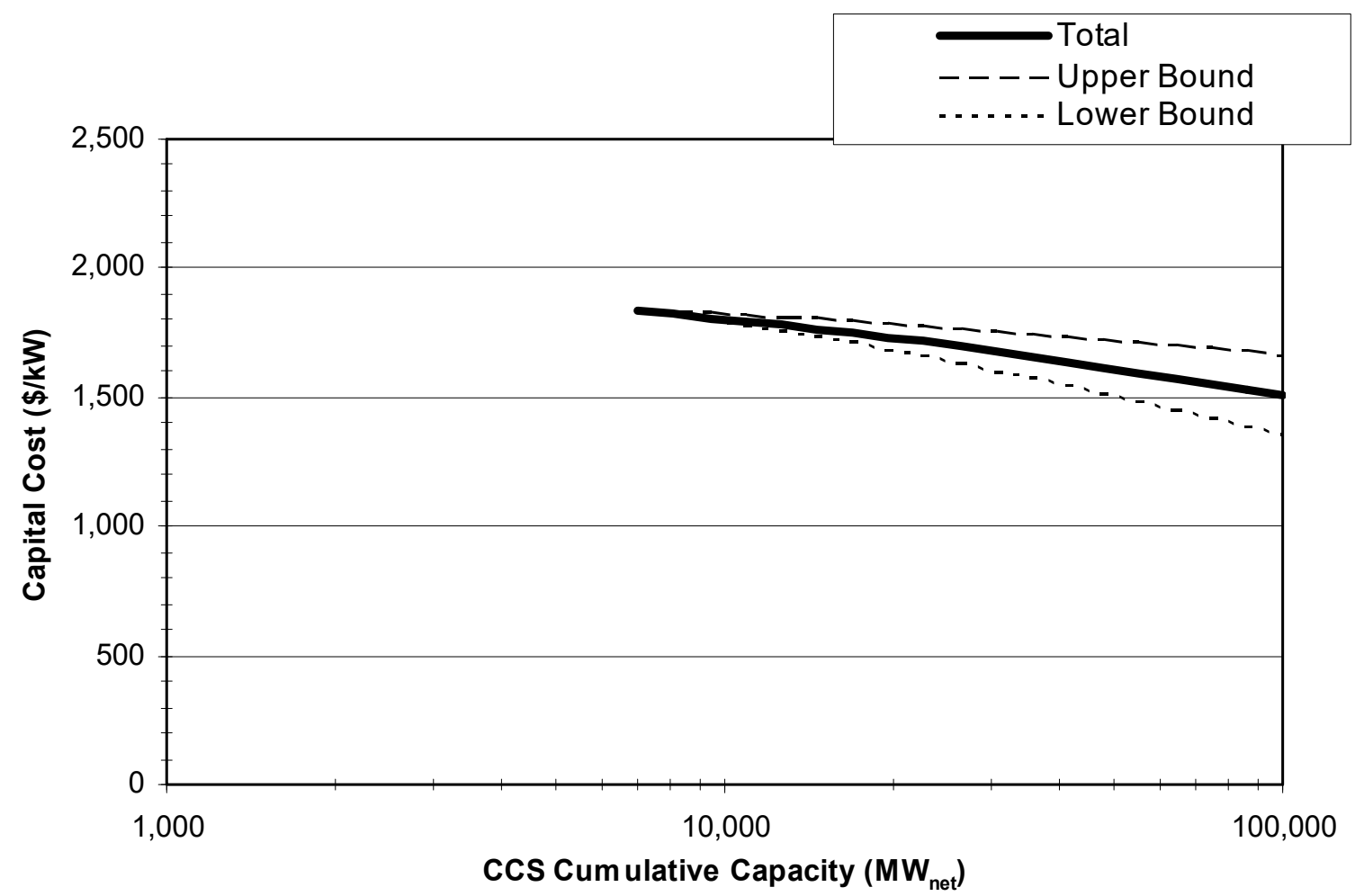

Figure C-2. Effect of learning rate uncertainties on cost projection for an advanced IGCC plant [56] 


\section{Appendix D: Example uncertainty in power plant cost using different analysis methods}

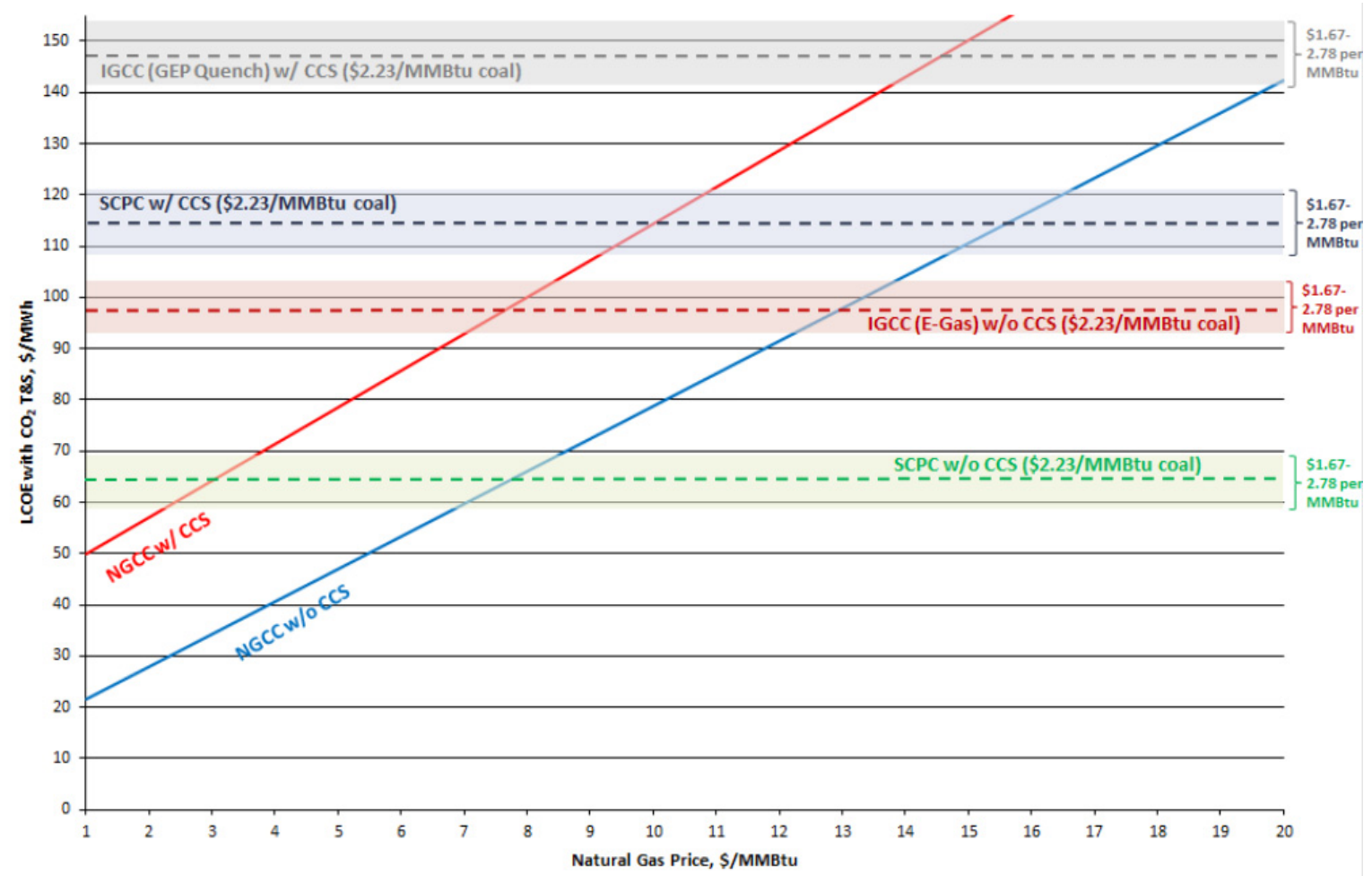

Figure D-1. LCOE sensitivity to fuel costs for NGCC, SCPC, and IGCC plants with and without carbon capture and storage (CCS) [43]

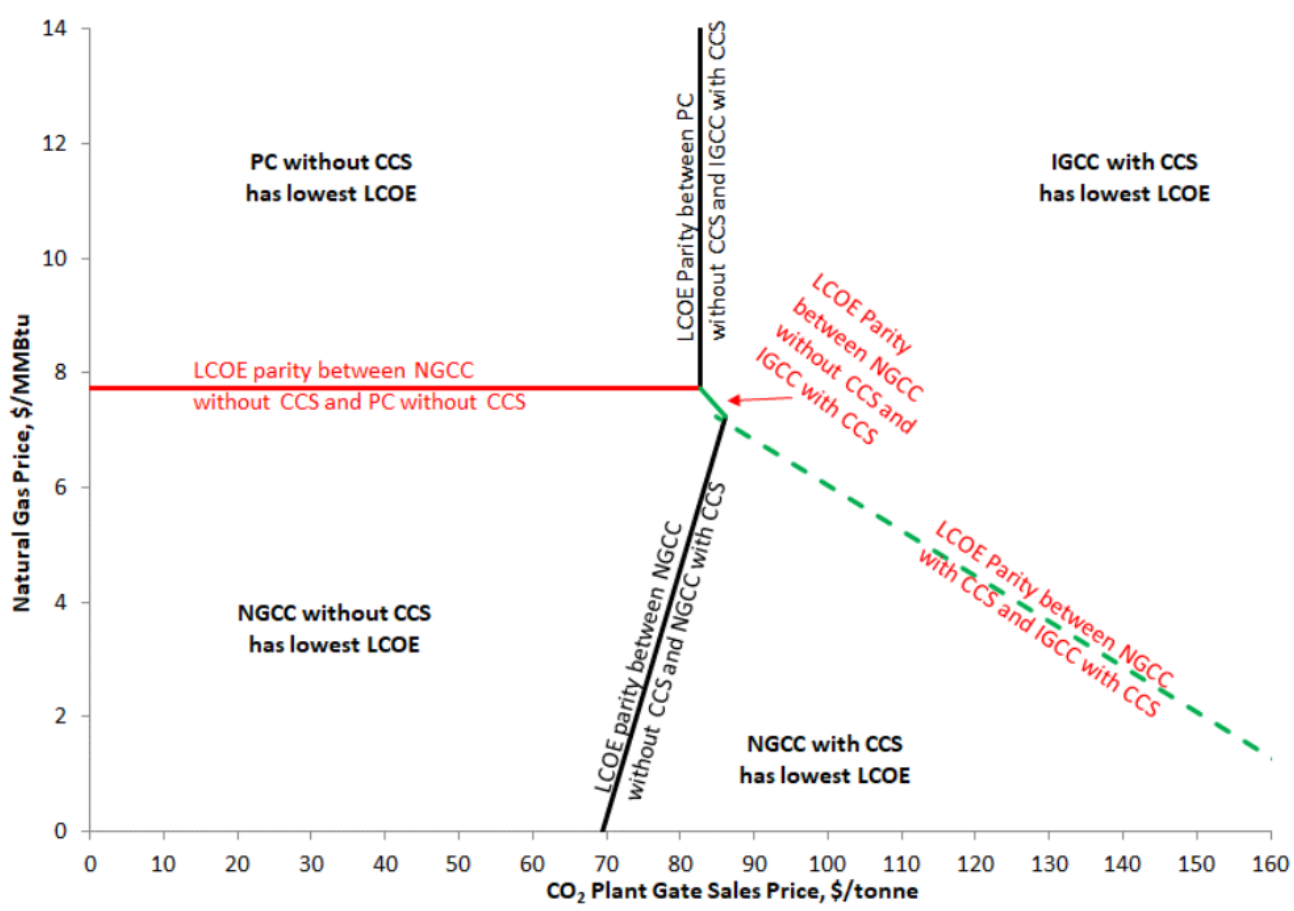

Figure D-2. Phase diagram plot showing the lowest-cost technology options at various natural gas and $\mathrm{CO}_{2} \mathrm{Sales}$ prices for NGCC, PC, and IGCC plants with and without CCS [43] 
Table D-1. Nominal values and uncertainty parameters assessed for the ammonia-based $\mathrm{CO}_{2}$ capture system [63]

\begin{tabular}{|c|c|c|c|c|}
\hline Parameter & Units & $\begin{array}{l}\text { Nominal } \\
\text { (x) }\end{array}$ & Values (or $\sigma$ as $\%$ of $x$ ) & References \\
\hline \multicolumn{5}{|l|}{ Capture System Performance } \\
\hline Chilling Loads Required at $<283^{\circ} \mathrm{K}$ & Tons Refrigeration & 103,000 & $\operatorname{Normal}(\mathrm{x}, 10 \%)$ & Author Estimate \\
\hline Chilling Loads Required at $283^{\circ} \mathrm{K}$ to $302^{\circ} \mathrm{K}$ & Tons Refrigeration & 21,333 & $\operatorname{Normal}(\mathrm{x}, 10 \%)$ & Author Estimate \\
\hline Chiller Electrical Use, $276^{\circ} \mathrm{K}$ Water Product & $\mathrm{kW} /$ Ton Refrigeration & 0.55 & Triangular $(0.47,0.55,0.60)$ & {$[216]$} \\
\hline Chiller Electrical Use, $280^{\circ} \mathrm{K}$ Water Product & kW/Ton Refrigeration & 0.47 & Triangular $(0.47,0.47,0.55)$ & {$[216]$} \\
\hline $\mathrm{CO}_{2}$ Regeneration Heat Requirement & $\mathrm{kJ} / \mathrm{kg} \mathrm{CO} 2$ & 2293 & $\operatorname{Normal}(\mathrm{x}, 10 \%)$ & Author Estimate \\
\hline Pumping Head & $\mathrm{kPa}$ & 207 & Triangular(150,207,250) & {$[217]$} \\
\hline Pump Efficiency & $\%$ & 75 & Uniform $(70,75)$ & {$[217]$} \\
\hline$\Delta \mathrm{P}$ Across $\mathrm{CO}_{2}$ Capture System & $\mathrm{kPa}$ & 20.7 & Triangular(14,26,30) & {$[217]$} \\
\hline Blower Efficiency & $\%$ & 75 & Uniform $(70,75)$ & {$[217]$} \\
\hline $\mathrm{CO}_{2}$ Compression, 27.5 bar to 152.7 bar & $\mathrm{kWh} / \mathrm{kg} \mathrm{CO} 2$ & 0.03 & Triangular $(0.028,0.03,0.032)$ & \\
\hline \multicolumn{5}{|l|}{ Capture System Cost } \\
\hline Reference Chilling Equipment Costs (PFC) & $\begin{array}{c}\text { \$2007/Ton } \\
\text { Refrigeration }\end{array}$ & 441 & Uniform $(0.7 x-1.3 x)$ & Author Estimate \\
\hline Reference IECM Costs (PFC) & $\$ 2007$ & 251.9 & Uniform $(0.7 x-1.3 x)$ & Author Estimate \\
\hline Reference Aspen Icarus $®$ Costs (PFC) & $\$ 2007$ & 59.4 & Uniform $(0.7 x-1.4 x)$ & Author Estimate \\
\hline $\mathrm{CO}_{2}$ Absorber Costs (PFC) & $\$ 2007$ & 105.1 & Uniform $(0.7 x-2.5 x)$ & {$[218]$} \\
\hline General Facilities Capital & $\%$ of PFC & $1.57^{1}$ & Normal(x, 10\%) & [219] \\
\hline Eng. \& Home Office Fees & $\%$ of $\mathrm{PFC}$ & $9.37^{1}$ & Triangular $(0.7 x, 1 x, 1.5 x)$ & {$[219]$} \\
\hline Project Contingency Cost & $\%$ of $\mathrm{PFC}$ & $16.38^{1}$ & Normal(x, 20\%) & {$[219]$} \\
\hline Process Contingency Cost & $\%$ of $\mathrm{PFC}$ & $4.67^{1}$ & Normal(x, 30\%) & {$[219]$} \\
\hline $\mathrm{CO}_{2}$ System Fixed O\&M/Year & \$2007 Million/year & 8.0 & Uniform $(0.7 x-1.3 x)$ & Author Estimate \\
\hline $\mathrm{CO}_{2}$ System Variable O\&M/Year + TS\&M & \$2007 Million/year & 14.0 & Uniform $(0.7 x-1.3 x)$ & Author Estimate \\
\hline \multicolumn{5}{|l|}{ Plant Financing \& Utilisation } \\
\hline Power Plant Fixed Charge Factor & fraction & 0.143 & Uniform $(0.130,0.180)$ & {$[220]$} \\
\hline Power Plant Levelised Capacity Factor & -- & 0.75 & Uniform $(0.65,0.85)$ & {$[220]$} \\
\hline
\end{tabular}




\section{Appendix E: EIA NEMS model assumptions for power plant learning rates}

Table E-1. Learning parameters for new generating technology components in the EIA NEMS model [221]

\begin{tabular}{|c|c|c|c|c|c|c|}
\hline Technology Component & $\begin{array}{l}\text { Period } 1 \\
\text { Learning } \\
\text { Rate }\end{array}$ & $\begin{array}{l}\text { Period } 2 \\
\text { Learning } \\
\text { Rate }\end{array}$ & $\begin{array}{l}\text { Period } 3 \\
\text { Learning } \\
\text { Rate }\end{array}$ & $\begin{array}{l}\text { Period } 1 \\
\text { Doublings }\end{array}$ & $\begin{array}{l}\text { Period } 2 \\
\text { Doublings }\end{array}$ & $\begin{array}{l}\text { Minimum Total } \\
\text { Learning by } 2025\end{array}$ \\
\hline Pulverized Coal & & & $1 \%$ & & & $5 \%$ \\
\hline Combustion Turbine - conventional & & & $1 \%$ & & & $5 \%$ \\
\hline Combustion Turbine - advanced & & $10 \%$ & $1 \%$ & & 5 & $10 \%$ \\
\hline $\mathrm{HRSG}^{1}$ & & & $1 \%$ & & & $5 \%$ \\
\hline Gasifier & & $10 \%$ & $1 \%$ & & 5 & $10 \%$ \\
\hline Carbon Capture/Sequestration & $20 \%$ & $10 \%$ & $1 \%$ & 3 & 5 & $20 \%$ \\
\hline Balance of Plant - IGCC & & & $1 \%$ & & & $5 \%$ \\
\hline Balance of Plant - Turbine & & & $1 \%$ & & & $5 \%$ \\
\hline Balanceof Plant - Combined Cyde & & & $1 \%$ & & & $5 \%$ \\
\hline Fuel Cell & $20 \%$ & $10 \%$ & $1 \%$ & 3 & 5 & $20 \%$ \\
\hline Advanced Nuclear & $5 \%$ & $3 \%$ & $1 \%$ & 3 & 5 & $10 \%$ \\
\hline Fuel prep - Biomass IGCC & $20 \%$ & $10 \%$ & $1 \%$ & 3 & 5 & $20 \%$ \\
\hline Distributed Generation - Base & & $5 \%$ & $1 \%$ & & 5 & $10 \%$ \\
\hline Distributed Generation - Peak & & $5 \%$ & $1 \%$ & & 5 & $10 \%$ \\
\hline Geothermal & & $8 \%$ & $1 \%$ & & 5 & $10 \%$ \\
\hline Municipal Solid Waste & & & $1 \%$ & & & $5 \%$ \\
\hline Hydropower & & & $1 \%$ & & & $5 \%$ \\
\hline Wind & & & $1 \%$ & & & $1 \%$ \\
\hline Wind Offshore & $20 \%$ & $10 \%$ & $1 \%$ & 3 & 5 & $20 \%$ \\
\hline Solar Thermal & $20 \%$ & $10 \%$ & $1 \%$ & 3 & 5 & $20 \%$ \\
\hline Solar PV & $15 \%$ & $8 \%$ & $1 \%$ & 3 & 5 & $20 \%$ \\
\hline
\end{tabular}

The NEMS model assumptions above are not accompanied by supporting data and appear to reflect expert judgments based on a review of the experience curve and learning rate literature. In addition, NEMS uses several other factors to estimate future overnight costs, including a "technological optimism" factor - a multiplier of 1.0 or more, applied to the first four units of a new, unproven design to compensate for the tendency to underestimate the actual cost of a first-of-a-kind technology [59]. 


\section{Appendix F: Overview of openly-available, highly transparent, and detailed techno-economic studies for each industrial sector}

To ensure high-quality techno-economic evaluations, a strong level of detailed technical and cost knowledge of the industrial plant without $\mathrm{CO}_{2}$ capture and with the reference $\mathrm{CO}_{2}$ capture technology is required. Developing such detailed basis can, in practice, be challenging as industrial sectors are very different from one another and that, even within an industrial sector, industrial plants can differ significantly from one to the another.

Several efforts to develop such detailed studies have been undertaken over the past decades. Furthermore, these studies have also performed detailed evaluation of reference industrial plants and reference $\mathrm{CO}_{2}$ capture technologies which can be used as base case in comparative assessment. The following subsections provide an overview and discuss openly-available, highly transparent and detailed techno-economic studies ${ }^{25}$ for each industrial sector. To maximise potential further use of this benchmark basis review, the key characteristics, assumptions, and results of each study are summarised in the Supplementary Information of Roussanaly et al. [4] for all sectors. As $\mathrm{CO}_{2}$ transport and storage costs are very case-specific, as illustrated in section 2.3.3, the $\mathrm{CO}_{2}$ avoidance

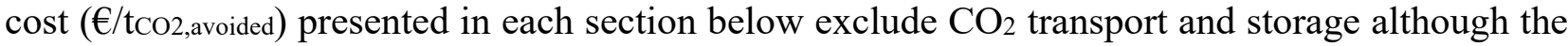
supplementation information includes both $\mathrm{CO}_{2}$ avoidance cost without and with $\mathrm{CO}_{2}$ transport and storage when available in the corresponding study.

Overall, while several detailed studies exist on CCS from industrial sectors, it is worth noting that a significant number of these studies have been published by IEAGHG or H2020 EU projects. Most of the key sectors include at least one detailed study apart from the petrochemical, waste-to-energy, and the offshore oil and gas sectors for which only semi-detailed techno-economic studies are available in literature. Finally, it is worth noting that most of these studies are for European locations, often Netherlands, and would need to be adapted for other continents/countries. As such additional studies representing more regional specificity (technology, cost, raw material specificity, local utilities conditions...) would be beneficial to the CCS from industry community.

\section{F.1 Iron and steel mill}

In practice, one detailed techno-economic study of blast furnace based-steelmaking plant with and without reference post-combustion MEA-based capture has been published by IEAGHG [78]. The reference plant considered was based on a new build integrated steel mill located in the coastal region of Western Europe producing $4 \mathrm{Mt} / \mathrm{y}$ of hot-rolled coil (HRC). While the steel mill consists of 12 major processes and various auxiliaries, nearly $90 \%$ of the plant's $\mathrm{CO}_{2}$ emissions comes from five units: hot stoves, power plant, sinter plant, coke ovens' underfired heaters and lime kilns.

The IEAGHG study established two benchmarked points for $\mathrm{CO}_{2}$ capture from an iron and steel plant. The first one, referred as Case $2 \mathrm{~A}$ case, investigated MEA-based $\mathrm{CO}_{2}$ capture from the flue gases of the hot stoves and the steam generation plant. The second one, Case $2 \mathrm{~B}$ case, investigated MEA-based $\mathrm{CO}_{2}$ capture from the flue gases of the underfire heaters of the coke oven batteries, hot stoves, lime kiln, and steam generation. These scenarios result in a reduction of 50 and $60 \%$ of the overall plant emissions. Compared to the reference levelised cost of HRC for the plant without capture ( $429 €_{2010} / \mathrm{t}_{\mathrm{HRC}}$ ), the cases with capture resulted in costs of 487 and $506 €_{2010} / \mathrm{t}_{\mathrm{HRC}}$ respectively. Based on this increase and the avoided $\mathrm{CO}_{2}$ emissions, a $\mathrm{CO}_{2}$ avoidance cost of 55 $€_{2010} / \mathrm{tCO}$,avoided was estimated for the case $2 \mathrm{~A}$, which achieve $50 \%$ avoided $\mathrm{CO}_{2}$ emissions from

\footnotetext{
${ }^{25}$ Transparent studies are defined as reports providing in-depth level of details of the technical and cost assessments of both the industrial plant with and without mature $\mathrm{CO}_{2}$ capture technology. Journal papers are thus excluded from this screening.
} 


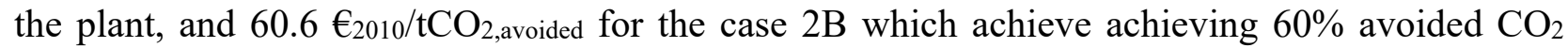
emissions from the plant.

\section{F.2 Cement}

In the past years, a strong focus has been set on reducing $\mathrm{CO}_{2}$ emissions from cement and, as such, several detailed techno-economic studies of cement with and without reference post-combustion MEA-based $\mathrm{CO}_{2}$ capture has been published by IEAGHG [79] and CEMCAP [222] umbrellas. In both cases, the cement plants considered correspond to the ECRA base cement plant producing with a clinker capacity of 3,000 t/d. However, there are a few differences between both studies. To account for air leakage which periodically appears in cement plant and which are only fixed every once or twice a year, the CEMCAP project included two periods used for design and cost evaluations ${ }^{26}$. Another key difference between both studies is the decision of how to produce the steam required to regenerate the absorbed $\mathrm{CO}_{2}$. While waste heat is recovered to supply around $7 \%$ of the heat need in both cases, the IEAGHG study considered a coal-based CHP plant or a NGCC to supply the remaining heat requirement, while CEMCAP assumed a natural gas boiler as well as different alternative strategies. While all these strategies can be valid decisions, these differences might have an impact on the cost. For example, an NGCC case that assumes the excess electricity could be sold at $80 €_{2014} / \mathrm{MWh}^{27}$ results in a low cost of steam as it gets indirectly subsidized by the high profit on the electricity sale. Thus, the strategies and assumptions in the IEAGHG study resulted on a $\mathrm{CO}_{2}$ avoidance cost of $52.4 €_{2013} / \mathrm{tCO}_{2, \text { avoided }}$ for the NGCC case and 102.9 $€_{2013} / \mathrm{tCO}_{2}$,avoided for the CHP case. Meanwhile, CEMCAP assumptions resulted in a $\mathrm{CO}_{2}$ avoidance cost of $80.2 €_{2014} / \mathrm{tCO}_{2}$,avoided when considering steam production based on a natural gas boiler.

\section{F.3 Refinery}

Refineries are the third contributor to industrial emissions. However, CCS from refineries can be challenging to assess due to heterogeneity of refineries, needs to retrofit in a space constraint plant, and the high number of $\mathrm{CO}_{2}$ emissions point sources. The ReCAP study [93] is the only extensive study published on the techno-economic performances of implementing CCS from a refinery. This study evaluated the design, integrations, and techno-economic performances of retrofitting $\mathrm{CO}_{2}$ capture into four different generic refineries: 1) a simple refinery with a nominal capacity of 100 $000 \mathrm{bbl} / \mathrm{d}$ 2) a medium complexity refinery with a nominal capacity of $220000 \mathrm{bbl} / \mathrm{d} \mathrm{3}$ ) a highly complex refinery with nominal capacity of $220000 \mathrm{bbl} / \mathrm{d}$ 4) a highly complex refinery with a nominal capacity of $350000 \mathrm{bbl} / \mathrm{d}$. Furthermore, as refineries are characterized by the large number of stacks with flue gases of varying $\mathrm{CO}_{2}$ concentration and sulphur content, multiple cases were considered for each refinery scenario. The results of the cost evaluation of the $16 \mathrm{CO}_{2}$ capture cases resulted in costs of retrofitting $\mathrm{CO}_{2}$ capture with an MEA-based process lies between 145.5 and $189.4 €_{2015 \mathrm{Q} 4} / \mathrm{tCO}_{2}$,avoided. These estimates are significantly larger than estimates available in the literature on $\mathrm{CO}_{2}$ capture for other sources (natural gas and coal power generation, cement, steel, etc.) for three main reasons: 1) the inclusion of the retrofit costs such as interconnection costs 2) the utilities cost is based on the installation of an additional CHP plant, cooling water towers and wastewater plant which are all designed with significant spare capacity in some cases (up to $30 \%$ overdesign). 3) Most of the $\mathrm{CO}_{2}$ capture cases considered include small to medium $\mathrm{CO}_{2}$ emission point sources and in some cases low to medium flue gas $\mathrm{CO}_{2}$ content and/or significant amount of sulphur.

\footnotetext{
${ }^{26}$ The two time periods were (1) a low air leak time period in which the flue gas contain $22 \%$ vol of $\mathrm{CO}_{2}$ and lasting 6 months every year (2) a high air leak time period in which the flue gas contain $18 \%$ vol of $\mathrm{CO}_{2}$ and lasting 6 months every year. This aspect is meant to more accurately represent the conditions of a cement plant, but results in higher CAPEX and OPEX compared to an evaluation considering only a low or an average air leak scenario.

${ }^{27}$ Average electricity price in the European Union area was 58.1 €/MWh in 2014
} 


\section{F.4 Hydrogen}

While auto-thermal reforming is key to large-scale production of hydrogen, steam methane reforming is the leading technology for production of hydrogen from natural gas and light gas. A detailed techno-economic study of SMR-based hydrogen production plant with and without CCS has been published by IEAGHG [86]. This study evaluated the design, performances, and cost of a new build hydrogen production plant located in the Netherlands producing $100000 \mathrm{Nm}^{3} / \mathrm{h}$ of hydrogen using natural gas as a feedstock.

In such a plant, the $\mathrm{CO}_{2}$ can be captured at three different locations: 1) synthesis gas before the $\mathrm{H}_{2}$ Pressure Swing Adsorption (PSA) 2) tail gas after $\mathrm{H}_{2}$ PSA 3) flue gas of the SMR furnace. While the two first low locations tend to result in lower cost due to the high $\mathrm{CO}_{2}$ partial pressure, a main drawback of CCS from these two locations is that only $60 \%$, approximately, of the plant $\mathrm{CO}_{2}$ emissions can be captured. On the other hand, CCS from the SMR furnace can reduce the emissions of the hydrogen plant beyond $90 \%$, although the capture cost could be higher than in the other two options.

Based on the considered plant, the IEAGHG study established three benchmark points for $\mathrm{CO}_{2}$ capture based on chemical absorption: 1) $\mathrm{CO}_{2}$ capture from shifted syngas using MDEA 2) $\mathrm{CO}_{2}$ capture from PSA tail gas using MDEA 3) $\mathrm{CO}_{2}$ capture from flue gas using MEA. These scenarios result in a reduction of 54,52, and $89 \%$ of the overall plant emissions. Compared to the reference levelised cost of hydrogen without capture $\left(11.4 \mathrm{c}_{2014 \mathrm{Q}} / \mathrm{Nm}^{3} \mathrm{H} 2\right)$, the cases with capture resulted in costs of $13.5,14.2$, and $16.5 \mathrm{c}_{2014 \mathrm{Q}} / \mathrm{Nm}^{3} \mathrm{H} 2$ respectively. Based on these LCOH increases and the avoided $\mathrm{CO}_{2}$ emissions, $\mathrm{CO}_{2}$ capture costs of 36.4 and $55.5 €_{2014 \mathrm{Q}} / \mathrm{tCO}_{2}$,avoided were estimated for first two cases, and $58.7 €_{2014 \mathrm{Q} 4} / \mathrm{tCO}_{2 \text {,avoided }}$ for the third case which is the only one enabling lowcarbon footprint hydrogen.

\section{F.5 Ammonia/ urea and methanol}

Currently, $60 \%$ of hydrogen syngas produced are used for production of ammonia/urea and methanol. Building on its hydrogen study, IEAGHG published a study on ammonia/urea and methanol production with CCS [83]. This study investigated the performances and cost of a new built plant producing ammonia/urea or methanol without and with CCS. In both cases, the industrial complex was based on the integration of a syngas plant based on SMR from natural gas. In the first case, the syngas plant was integrated in an ammonia plant with a $1350 \mathrm{t} / \mathrm{d}$ nominal capacity. Around $95 \%$ of the produced ammonia was considered to be further converted downstream in an ammonia plant $(2260 \mathrm{t} / \mathrm{d})$ using $\mathrm{CO}_{2}$ captured from the syngas plant. In the second case, the syngas plant was integrated in a methanol plant with a nominal capacity of $5000 \mathrm{t} / \mathrm{d}$. It is important to note that in both cases, most of the carbon entering the processes end up in the final products: $69.3 \%$ in the ammonia/urea case and $79.3 \%$ in the methanol case. The cases with $\mathrm{CO}_{2}$ capture thus aim at capturing around $90 \%$ of the remaining $\mathrm{CO}_{2}$ emissions through post-combustion $\mathrm{MEA}$-based $\mathrm{CO}_{2}$ capture from the SMR flue gas.

For the ammonia/urea plant, the levelised cost of urea increases from 257.3 to $280.3 €_{2014 \mathrm{Q} 4 / \mathrm{t} \text { once }}$ CCS is implemented. Based on the specific emissions reduction, the corresponding cost of $\mathrm{CO}_{2}$ capture is 75 or $83.9 €_{2014 \mathrm{Q}} / \mathrm{tCO}_{2}$,avoided depending on assumed electricity source (natural gas or a coal power plant). It is worth noting that in the case with capture, part of the $\mathrm{CO}_{2}$ captured is used to reach a total urea production of $2380 \mathrm{t} / \mathrm{d}$.

For the methanol plant, the levelised cost of methanol increase from 275.1 to $298.9 €_{2014 \mathrm{Q} 4 / \mathrm{t}}$ by implementing CCS. The obtained costs of $\mathrm{CO}_{2}$ resulting of thus 70.6 and $78.9 €_{2014 \mathrm{Q}} / \mathrm{tCO}$,avoided depending on the electricity source (natural gas or a coal power plant), thus slightly lower than in the urea case. 
One important aspect to note is that as most of the carbon entering the processes end up in the final products and might be released to the atmosphere through the product (or one of its derivates) use $[223,224]$, the climate impact of the end product might only be moderately reduced through the implementation of CCS in this case.

\section{F.6 Pulp and board}

Although a large proportion of these is biogenic emissions, the pulp and board production sector contributes to nearly $5 \%$ of the $\mathrm{CO}_{2}$ emissions of the industrial sector. Furthermore, reducing these emissions is key in achieving the climate ambitions of certain countries as nearly $75 \%$ of the pulp and paper production is concentrated in ten countries. A detailed techno-economic study on retrofitting CCS on pulp and board mills was published by IEAGHG [82]. This study assessed two hypothetical reference mills situated in the west coast of Finland. The first one is a pulp mill producing 800,000 air-dried tonne (adt) per year of bleached softwood pulp (BSP). The second one is an integrated pulp and board mill which produces 400,000 adt of board per year and 740,000 adt/y of BSP. For both plants, the main sources responsible for the $\mathrm{CO}_{2}$ emissions of the plant are the recovery boiler (REC), the multi-fuel boiler (MFB), and the lime kiln (LK). These three sources are responsible for respectively 76,14 , and $10 \%$ of the plant non-biogenic emissions. However, it is worth noting that each of these sources also emits 24 tonnes of biogenic $\mathrm{CO}_{2}$ emissions per tonne of non-biogenic $\mathrm{CO}_{2}$ emissions. For each plant, retrofitting an MEA-based post-combustion capture from these sources was evaluated on a stand-alone or combined basis thus resulting in six capture scenarios: 1) REC only 2) MFB only 3) LK only 4) REC + MFB 5) REC + LK 6) REC + MFB + LK.

While the cost of the pulp and board plants without capture results in a levelised cost of pulp of $522.6 €_{2015} / \mathrm{adt}$, this cost varies between 543 and $676 €_{2015} /$ adt for the pulp mill and 545 and 714 $€_{2015}$ adt for the pulp and board mill depending on the capture case considered. This results in $\mathrm{CO}_{2}$

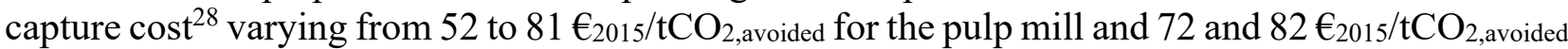
for the pulp and board mill. In general, the scenarios based on $\mathrm{CO}_{2}$ capture from the REC standalone or combined with other sources results in the lowest cost. It is worth noting that despite the retrofit cost and the shorter operation duration for the $\mathrm{CO} 2$ capture facility ( 15 years), the $\mathrm{CO}_{2}$ capture cost remains rather low as excess steam produced by the mill is assumed to supply the required heat demand for $\mathrm{CO}_{2}$ regeneration.

\footnotetext{
${ }^{28}$ Biogenic and non-biogenic emissions are here considered alike.
} 


\section{Appendix G: Other methods for calculating $\mathrm{CO}_{2}$ avoidance cost and their associated assumptions}

The "net present value" and "annualisation" methods for calculation of CAC are presented in Equation 11 [225] and Equation 12 [226], while a summary of assumptions required to ensure the validity of each $\mathrm{CO}_{2}$ avoidance cost calculation methods is presented in Table G-1. More details on the links between the different calculation methods and the associated assumptions can be found in Roussanaly [99].

$$
C A C=\frac{N P V_{\mathrm{CCS}}}{\sum_{i} \frac{\dot{M}_{\mathrm{CO} 2, \text { avoided }, \mathrm{i}}}{(1+r)^{i}}}
$$

Where:

- $\quad N P V_{\mathrm{CCS}}$ is the net present value of total annual CCS costs (which may vary from year to year).

- $\dot{M}_{\mathrm{CO} 2 \text {,avoided,i }}$ is the mass of $\mathrm{CO}_{2}$ avoided by CCS implementation in year $\mathrm{i}$.

- $r$ is the discount rate.

$$
C A C=\frac{I_{\mathrm{CCS}, \mathrm{a}}+O_{\mathrm{CCS}}}{\dot{M}_{\mathrm{CO} 2, \text { avoided }}}
$$

Where:

- $I_{\mathrm{CCS}, \mathrm{a}}$ is the annualised investment cost of CCS.

- $O_{\mathrm{CCS}}$ is the annual operating cost of CCS.

- $\dot{M}_{\mathrm{CO} 2 \text {,avoided }}$ is the annual reduction in $\mathrm{CO}_{2}$ emissions due to CCS for a plant producing the same amount of product(s) with and without CCS.

Table G-1. Summary of assumptions required to ensure the validity of each $\mathrm{CO}_{2}$ avoidance cost calculation

\begin{tabular}{|c|c|c|c|}
\hline Assumption & $\begin{array}{l}\text { "Exhaustive" } \\
\text { method }\end{array}$ & $\begin{array}{l}\text { "Net present } \\
\text { value" method }\end{array}$ & $\begin{array}{c}\text { "Annualisation" } \\
\text { method }\end{array}$ \\
\hline $\begin{array}{l}\text { Production of industrial plant not affected by CCS } \\
\text { implementation }\end{array}$ & 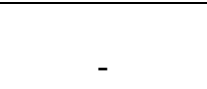 & Yes & Yes \\
\hline $\begin{array}{l}\text { Additional costs and } \mathrm{CO}_{2} \text { emissions avoided due to } \mathrm{CCS} \\
\text { implementation can be assessed separately }\end{array}$ & - & Yes & Yes \\
\hline $\begin{array}{l}\text { Annual operating costs and } \mathrm{CO}_{2} \text { emissions avoided are } \\
\text { constant over project duration } \\
\mathrm{CO}_{2} \text { emissions linked to construction of the } \mathrm{CCS} \text { facility can }\end{array}$ & - & - & Yes \\
\hline be neglected or excluded & - & - & Yes \\
\hline
\end{tabular}
methods. For the "exhaustive" method none of these assumptions are required [99] 


\section{Appendix H: Definitions of Technology Readiness Levels}

Table H-1. Definitions of TRL by different institutes

\begin{tabular}{|c|c|c|c|c|c|}
\hline TRL & $\begin{array}{l}\text { European Commission, } \\
\text { Horizon2020 [227] }\end{array}$ & UK government [228] & NASA [229] & EPRI [142] & IEA [230] \\
\hline 1 & Basic principles observed & $\begin{array}{l}\text { Basic principles } \\
\text { established }\end{array}$ & $\begin{array}{l}\text { Basic principles observed and } \\
\text { reported }\end{array}$ & Basic principles, observed, initial concept & $\begin{array}{l}\text { Initial Idea: basic principles } \\
\text { have been defined }\end{array}$ \\
\hline 2 & $\begin{array}{l}\text { Technology concept } \\
\text { formulated }\end{array}$ & Invention and Research & $\begin{array}{l}\text { Technology concept and/or } \\
\text { application formulated }\end{array}$ & Formulation of the application & $\begin{array}{l}\text { Application formulated: concept } \\
\text { and application of solution have } \\
\text { been formulated }\end{array}$ \\
\hline 3 & $\begin{array}{l}\text { Experimental proof of } \\
\text { concept }\end{array}$ & Proof of concept & $\begin{array}{l}\text { Analytical and experimental } \\
\text { critical function and/or } \\
\text { characteristic proof of concept }\end{array}$ & Proof of concept, tests, component level & $\begin{array}{l}\text { Concept needs validation: } \\
\text { Solution needs to be prototyped } \\
\text { and applied }\end{array}$ \\
\hline 4 & $\begin{array}{l}\text { Technology validated in the } \\
\text { lab }\end{array}$ & Bench scale & $\begin{array}{l}\text { Component and/or breadboard } \\
\text { validation in laboratory } \\
\text { environment }\end{array}$ & System validation in a laboratory environment & $\begin{array}{l}\text { Early prototype: prototype } \\
\text { proven in test conditions }\end{array}$ \\
\hline 5 & $\begin{array}{l}\text { Technology validated in } \\
\text { relevant environment }\end{array}$ & Pilot scale & $\begin{array}{l}\text { Component and/or breadboard } \\
\text { validation in relevant environment }\end{array}$ & Sub-system validation in a relevant environment & $\begin{array}{l}\text { Large prototype: Components } \\
\text { proven in conditions to be } \\
\text { deployed }\end{array}$ \\
\hline 6 & $\begin{array}{l}\text { Technology demonstrated in } \\
\text { relevant environment }\end{array}$ & Large scale & $\begin{array}{l}\text { System/subsystem model or } \\
\text { prototype demonstration in a } \\
\text { relevant environment }\end{array}$ & $\begin{array}{l}\text { Fully integrated pilot tested in a relevant } \\
\text { environment }\end{array}$ & $\begin{array}{l}\text { Full prototype at scale: } \\
\text { prototype proven at scale in } \\
\text { conditions to be deployed }\end{array}$ \\
\hline 7 & $\begin{array}{l}\text { System prototype } \\
\text { demonstration in operational } \\
\text { environment }\end{array}$ & Inactive commissioning & $\begin{array}{l}\text { System prototype demonstration in } \\
\text { a space environment }\end{array}$ & $\begin{array}{l}\text { Sub-scale demonstration, fully functional } \\
\text { prototype }\end{array}$ & $\begin{array}{l}\text { Pre-commercial demonstration: } \\
\text { solution working in expected } \\
\text { conditions }\end{array}$ \\
\hline 8 & $\begin{array}{l}\text { System complete and } \\
\text { qualified }\end{array}$ & Active commissioning & $\begin{array}{l}\text { Actual system completed and } \\
\text { "flight qualified" through test and } \\
\text { demonstration }\end{array}$ & $\begin{array}{l}\text { Commercial demonstration, full scale deployment } \\
\text { in final form }\end{array}$ & $\begin{array}{l}\text { First-of-a-kind commercial: } \\
\text { commercial demonstration, full } \\
\text { scale deployment in final form }\end{array}$ \\
\hline 9 & $\begin{array}{l}\text { Actual system proven in } \\
\text { operational environment }\end{array}$ & Operation & $\begin{array}{l}\text { Actual system "flight proven" } \\
\text { through successful mission } \\
\text { operations }\end{array}$ & Normal commercial service & $\begin{array}{l}\text { Commercial operation in } \\
\text { relevant environment: solution is } \\
\text { commercially available, needs } \\
\text { evolutionary improvement to } \\
\text { stay competitive }\end{array}$ \\
\hline 10 & & & & & $\begin{array}{l}\text { Integration at scale: solution is } \\
\text { commercial and competitive but } \\
\text { needs further integration efforts }\end{array}$ \\
\hline 11 & & & & & $\begin{array}{l}\text { Proof of stability: predictable } \\
\text { growth }\end{array}$ \\
\hline
\end{tabular}




\section{Appendix I: Mathematical representations of uncertainty analysis}

In OAT sensitivity analysis, the model output vector $\mathbf{y}$ is only evaluated against a minimum and maximum value of an input parameter $\mathrm{x}_{\mathrm{i}}$. It assumes a base case vector of input parameters $\boldsymbol{x}^{\mathbf{0}}$ and a sensitivity perturbation ( $\min / \max$, the plus/minus $10 \%$ ) of the input parameters $\boldsymbol{x}^{+}$. The difference between the base case and the sensitivity case is then $\Delta^{+} \boldsymbol{x}=\boldsymbol{x}^{+}-\boldsymbol{x}^{\mathbf{0}} \Delta^{+}$. The output of the sensitivity analysis is the delta between $y_{i}{ }^{+}$and $y_{i}{ }^{+0}$ :

$$
\Delta_{i}^{+} y=g\left(x_{i}+\Delta x_{i}^{+}, \mathbf{x}_{\sim i}^{0}\right)-g\left(\mathbf{x}^{0}\right)
$$

Where $\mathbf{x}_{\sim i}^{0}$ is the vector of all inputs other than $i$ (i.e., the inputs that are kept constant). A one-way sensitivity function $h_{i}\left(x_{i}\right)$ can be defined as:

$$
h_{i}\left(x_{i}\right)=g\left(x_{i} ; x_{\sim i}^{0}\right)
$$

A typical representation of one-way sensitivity analysis is by so-called spider-plots, where the sensitivity of the output values is plotted against changes in the vector of input values. To plot the changes to all input parameters in one graph, they need to be normalised (calculated as a percentage deviation) according to the function:

$$
h_{i}^{*}\left(x_{i}\right)=h_{i}\left(x_{i}\right)-g\left(\boldsymbol{x}^{o}\right)=g\left(x_{i} ; \boldsymbol{x}_{\sim i}^{0}\right)-g\left(\boldsymbol{x}^{o}\right)
$$




\section{Appendix J: Guideline for the characterisation of probability density functions by Hawer et al. [180]}

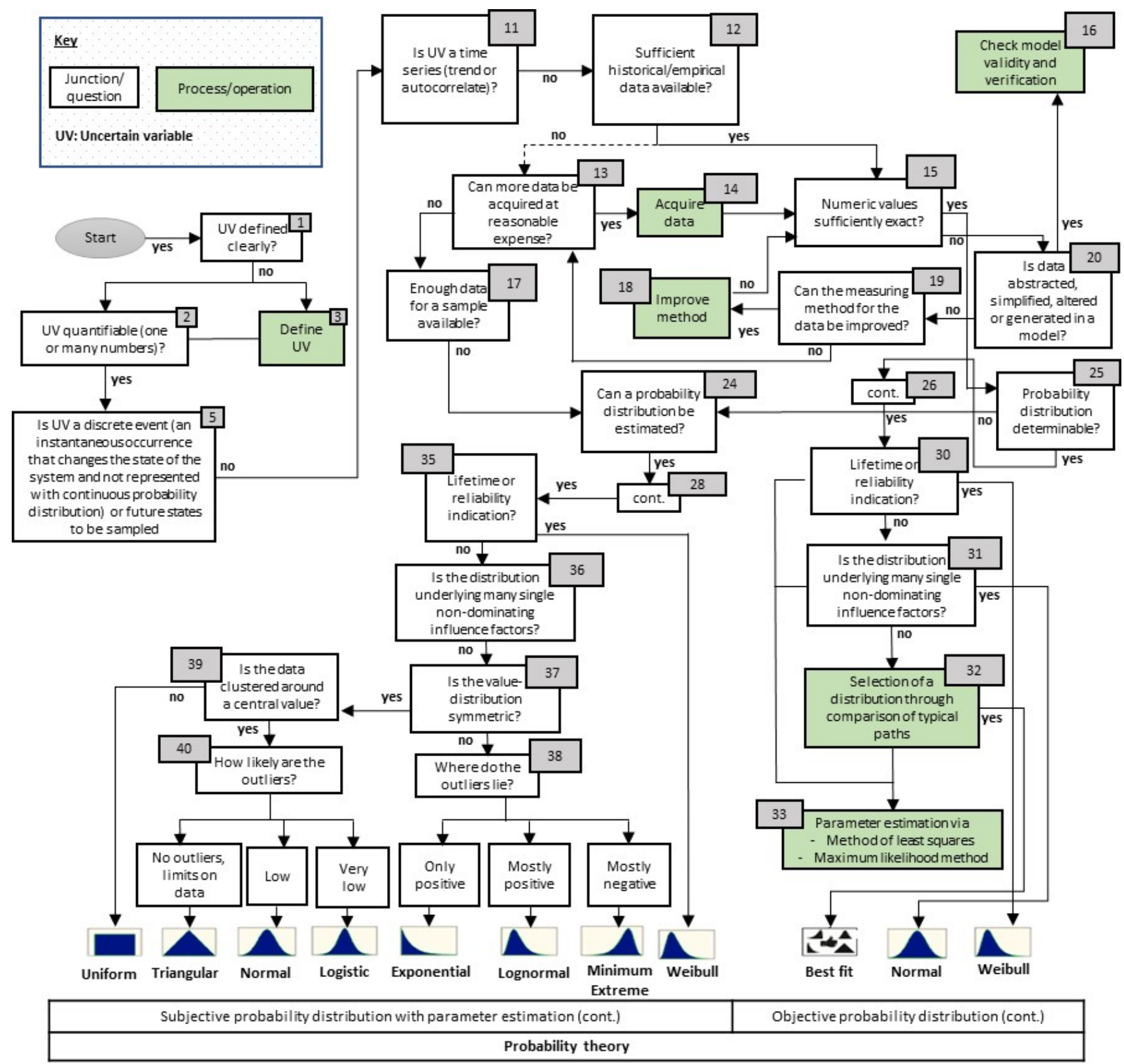

Figure J-1. Guideline for the classification and quantification of uncertainty, based on Hawer et al. [37]. UV is short for Uncertain Variable; the numbers in the boxes refer to the original numbering by Hawer et al [37], of which we used a subset. 


\section{References}

[1] IEAGHG. Costs Network. Available from: https://ieaghg.org/networks/costs-network. 2019.

[2] Rubin E, Booras G, Davison J, Ekstrom C, Matuszewski M, McCoy ST, et al. Toward a common method of the cost estimation for $\mathrm{CO}_{2}$ capture and storage at fossil fuel power plants.2013.

[3] Rubin ES. Improving cost estimates for advanced low-carbon power plants. International Journal of Greenhouse Gas Control. 2019;88:1-9.

[4] Roussanaly S, Berghout N, Fout T, Garcia M, Gardarsdottir S, Nazir SM, et al. Towards improved cost evaluation of Carbon Capture and Storage from industry. Accepted in Int J of Greenhouse Gas Control. 2021.

[5] van der Spek M, Fout T, Garcia M, Kuncheekanna VN, Matuszewski M, McCoy S, et al. Uncertainty analysis in the techno-economic assessment of $\mathrm{CO}_{2}$ capture and storage technologies. Critical review and guidelines for use. International Journal of Greenhouse Gas Control. 2020;100:103113.

[6] Rubin ES, Short C, Booras G, Davison J, Ekstrom C, Matuszewski M, et al. A proposed methodology for $\mathrm{CO}_{2}$ capture and storage cost estimates. International Journal of Greenhouse Gas Control. 2013;17:488503.

[7] US DOE. Technology Readiness Assessment Guide, DOE G413.3-4A, 9-15-2011, Office of Management, U.S. Department of Energy, Washington, D.C. . 2011.

[8] Rubin ES. Understanding the pitfalls of CCS cost estimates. International Journal of Greenhouse Gas Control. 2012;10:181-90.

[9] Rubin E, Mantripragada H, Zhai H. An Assessment of the NETL Cost Estimation Methodology, Report from Department of Engineering and Public Policy, Carnegie Mellon University Pittsburgh, PA to DOE/NETL, Pittsburgh, PA, April, 79p. 2016.

[10] NETL. Current and Future Technologies for Power Generation with Post-Combustion Carbon Capture, Final Report, National Energy Technology Laboratory, U.S. Department of Energy, Pittsburgh, PA. 2012.

[11] NETL. Techno-Economic Analysis of Integrated Gasification Fuel Cell Systems, National Energy Technology Laboratory, U.S. Department of Energy, Pittsburgh, PA. 2014.

[12] NETL. Current and Future IGCC Technologies: A Pathway Study Focused on Carbon Capture Advanced Power Systems R\&D Using Bituminous Coal, Revision 3, National Energy Technology Laboratory, U.S. Department of Energy, Pittsburgh, PA. 2015.

[13] Guandalini G, Romano MC, Ho M, Wiley D, Rubin ES, Abanades JC. A sequential approach for the economic evaluation of new $\mathrm{CO}_{2}$ capture technologies for power plants. International Journal of Greenhouse Gas Control. 2019;84:219-31.

[14] Taylor MR, Rubin ES, Hounshell DA. Effect of Government Actions on Technological Innovation for SO2 Control. Environmental Science \& Technology. 2003;37:4527-34.

[15] Rubin E, Booras G, Davidson J, Ekstrom C, Matuszewski M, McCoy S, et al. Toward a common method of cost estimation for $\mathrm{CO}_{2}$ capture and storage at fossil fuel power plants, IEAGHG 2013/TR02, Cheltenham, United Kingdom. 2013.

[16] NETL. Quality Guidelines for Energy System Studies: Cost Estimation Methodology for NETL Assessments of Power Plant Performance, National Energy Technology Laboratory, U.S. Department of Energy, Pittsburgh, PA. 2019.

[17] AACE. Conducting technical and economic evaluations- as applied for the process and utility industries, Recommended Practice No. 16R-90, AACE International, Morgantown, WV. 1991.

[18] EPRI. TAG TM Technical Assessment Guide Volume 1: Electricity Supply-1993, TR-102276-V1R1. Electric Power Research Institute, Palo Alto, CA. 1993.

[19] EPRI. Technical Assessment Guide (TAG ${ }^{\circledR}$ ) - Power Generation and Storage Technology Options, EPRI Product ID No. 1017465, Electric Power Research Institute, Palo Alto, CA. 2009. 
[20] NETL. Quality Guidelines for Energy System Studies Overview, National Energy Technology Laboratory, U.S. Department of Energy, Pittsburgh, PA. 2019.

[21] van der Spek M, Roussanaly S, Rubin ES. Best practices and recent advances in CCS cost engineering and economic analysis. International Journal of Greenhouse Gas Control. 2019;83:91-104.

[22] Gerdes K, Stevens R, Fout T, Fisher J, Hackett G, Shelton W. Current and Future Power Generation Technologies: Pathways to Reducing the Cost of Carbon Capture for Coal-fueled Power Plants. Energy Procedia. 2014;63:7541-57.

[23] NETL. Technology Learning Curve (FOAK to NOAK), Quality Guidelines for Energy System Studies, National Energy Technology Laboratory, U.S. Department of Energy, Pittsburgh, PA. 2014.

[24] BCG. Perspectives on Experience, Boston Consulting Group, Inc., Boston, MA. 1972.

[25] Wene C-O. Energy Technology Learning Through Deployment in Competitive Markets. The Engineering Economist. 2008;53:340-64.

[26] Rubin ES, Yeh S, Antes M, Berkenpas M, Davison J. Use of experience curves to estimate the future cost of power plants with $\mathrm{CO}_{2}$ capture. International Journal of Greenhouse Gas Control. 2007;1:188-97.

[27] Rubin ES, Azevedo IML, Jaramillo P, Yeh S. A review of learning rates for electricity supply technologies. Energy Policy. 2015;86:198-218.

[28] McDonald A, Schrattenholzer L. Learning rates for energy technologies. Energy Policy. 2001;29:25561.

[29] Thomassen G, Van Passel S, Dewulf J. A review on learning effects in prospective technology assessment. Renewable and Sustainable Energy Reviews. 2020;130:109937.

[30] Samadi S. The experience curve theory and its application in the field of electricity generation technologies - A literature review. Renewable and Sustainable Energy Reviews. 2018;82:2346-64.

[31] Louwen A, Junginger M, Krishnan A. Technological Learning in Energy Modelling: Experience Curves, Policy Brief, REflex: Analysis of the European Energy System, Utrecht University, Copernicus Institute of Sustainable Development, The Netherlands, 18p. 2018.

[32] Kittner N, Schmidt O, Staffell I, Kammen D. Chapter 8: Grid-scale Energy Storage. Technological Learning in the Transition to a Low-Carbon Energy System: Elsevier; 2020.

[33] Ramírez CA, Worrell E. Feeding fossil fuels to the soil: An analysis of energy embedded and technological learning in the fertilizer industry. Resources, Conservation and Recycling. 2006;46:75-93.

[34] Rubin E. The government role in technology innovation: lessons for the climate change policy agenda. In: Proc. of 10th Biennial Conference on Transportation Energy and Environmental Policy loTS, University of California, Davis, August., editor. 2005.

[35] Yeh S, Rubin ES. A review of uncertainties in technology experience curves. Energy Economics. 2012;34:762-71.

[36] Junginger M, van Sark W, Faaij A. Technological Learning in the Energy Sector: Lessons for Policy, Industry and Science, Edward Elgar Publishing Ltd, 352p. 2010.

[37] IEAGHG. Effects of Plant Location on the Costs of $\mathrm{CO}_{2}$ Capture. 2018/04, Cheltenham, United Kingdom. 2018.

[38] IECM. IECM Technical Documentation: Uncertainty Analysis, Report by the Integrated Environmental Control Model Team, Carnegie Mellon University, Pittsburgh, PA , to U.S. Dep't of Energy, National Energy Technology Laboratory, Pittsburgh, PA, January. Also, IECM Public Version software package, Available at: www.iecm-online.com. . 2019.

[39] ARENA. Commercial Readiness Index for Renewable Energy Sectors, Australian Renewable Energy Agency, Australian Government, Canberra. Available at: https://arena.gov.au/assets/2014/02/Commercial-Readiness-Index.pdf. 2014.

[40] Greig C, Garnett A, Oesch J, Smart S. Guidelines for Scoping and Estimating Early Mover CCS Projects, Milestone 5 Final Report, The University of Queensland, 19 June, 154p. 2014. 
[41] AACE. Cost estimate classification system - as applied in engineering, procurement, and construction for the process industries, Recommended Practice No. 18R-97, AACE International, Morgantown, WV. 2005.

[42] EIA. Electric Power Monthly, Table 6.07.A, Energy Information Administration, U.S. Department of Energy. Washington, DC. 2020.

[43] NETL. Cost and Performance Baseline for Fossil Energy Plants, Volume 1: Bituminous Coal and Natural Gas to Electricity, NETL-PUB-22638, National Energy Technology Laboratory, U.S. Department of Energy, Pittsburgh, PA. 2019.

[44] Cui Q, Lu H, Li C, Singh S, Ba L, Zhao X, et al. China baseline coal-fired power plant with postcombustion $\mathrm{CO}_{2}$ capture: 1 . Definitions and performance. International Journal of Greenhouse Gas Control. 2018;78:37-47.

[45] van der Spek M, Manzolini G, Ramirez A. New Approach to Techno-economic Assessment of Power Plants with Carbon Capture and Storage: The Inclusion of Realistic Dispatch Profiles To Calculate Technoeconomics of Part Load Operations. Energy \& Fuels. 2017;31:1047-9.

[46] Zappa W, Junginger M, van den Broek M. Is a $100 \%$ renewable European power system feasible by 2050? Applied Energy. 2019;233-234:1027-50.

[47] Black \& Veatch. Design and Cost Considerations for Advanced (Pre-Commercial) Power Plant Technologies, Report from Black \& Veatch to KeyLogic, Inc., Pittsburgh, PA, March, 40p. 2020.

[48] Ball M, Versteeg P. Factors impacting capital costs at SaskPower's Boundary Dam Integrated CCS Project, Proceedings of IEAGHG CCS Cost Network 2016 Workshop, 23-24 March 2016, Cambridge, Massachusetts, USA, Cheltenham, UK. 2016.

[49] CIAB. Learning by doing: The cost reduction potential for CCUS at coal-fired power plants. Coal Industry Advisory Board submission to the International Energy Agency. Paris, France. 2019.

[50] Aspentech. Aspen Capital Cost Estimator. Aspen Technology, Inc, Bedford, MA. 2017.

[51] Green D, Southhard M. Perry's Chemical Engineers' Handbook, 9th Edition: McGraw-Hill; 2019.

[52] Peters M, Timmerhaus K, West R. Plant Design and Economics for Chemical Engineers, 5th Edition: McGraw-Hill; 2003.

[53] Towler G, Sinnot R. Chemical Engineering Design: Principles, Practice and Economics of Plant and Process Design, 2nd ed. Oxford: Elsevier Ltd.; 2013.

[54] Abanades JC, Arias B, Lyngfelt A, Mattisson T, Wiley DE, Li $\mathrm{H}$, et al. Emerging $\mathrm{CO}_{2}$ capture systems. International Journal of Greenhouse Gas Control. 2015;40:126-66.

[55] Preston C, Bruce C, Monea M. An update on the integrated CCS project at SaskPower's Boundary Dam Power Station. Proc 14th International Conference on Greenhouse Gas Control Technologies, GHGT-14, 21-25 October, Melbourne, Australia2018.

[56] IEAGHG. Estimating future trends in the cost of $\mathrm{CO}_{2}$ capture technologies. 2006/06. Cheltenham, United Kingdom. 2006.

[57] van der Spek M, Ramirez A, Faaij A. Challenges and uncertainties of ex ante techno-economic analysis of low TRL $\mathrm{CO}_{2}$ capture technology: Lessons from a case study of an NGCC with exhaust gas recycle and electric swing adsorption. Applied Energy. 2017;208:920-34.

[58] EIA. The Electricity Market Module of the National Energy Modeling System: Model Documentation 2018, Energy Information Administration., U.S. Department of Energy, Washington, DC. 2018.

[59] EPRI. PRISM 2.0: Modeling Technology Learning for Electricity Supply Technologies Technical Report No. 3002000871, Prepared by Carnegie Mellon University, Electric Power Research Institute, Palo Alto, CA. 2013.

[60] Hollmann J. Improve your contingency estimates for more realistic project budgets, Chemical Engineering, pp.36-43. 2014.

[61] Morgan MG, Henrion M. Uncertainty: A Guide to Dealing with Uncertainty in Quantitative Risk and Policy Analysis. Cambridge: Cambridge University Press; 1990. 
[62] Zhai $\mathrm{H}$, Rubin E. Systems analysis of physical absorption of $\mathrm{CO}_{2}$ in ionic liquids for pre-combustion carbon capture. Environmental Science \& Technology. 2018;52:4996-5004.

[63] Versteeg P, Rubin ES. A technical and economic assessment of ammonia-based post-combustion $\mathrm{CO}_{2}$ capture at coal-fired power plants. International Journal of Greenhouse Gas Control. 2011;5:1596-605.

[64] Verdolini E, Anadón LD, Baker E, Bosetti V, Reis LA. Future Prospects for Energy Technologies: Insights from Expert Elicitations. Review of Environmental Economics and Policy. 2018;12:133-53.

[65] Rao AB, Rubin ES, Keith DW, Granger Morgan M. Evaluation of potential cost reductions from improved amine-based $\mathrm{CO}_{2}$ capture systems. Energy Policy. 2006;34:3765-72.

[66] van der Spek M, Arendsen R, Ramirez A, Faaij A. Model development and process simulation of postcombustion carbon capture technology with aqueous AMP/PZ solvent. International Journal of Greenhouse Gas Control. 2016;47:176-99.

[67] Morgan MG. Use (and abuse) of expert elicitation in support of decision making for public policy. Proceedings of the National Academy of Sciences. 2014;111:7176-84.

[68] Merkel T, Lin $\mathrm{h}$, Wei $\mathrm{X}$, et al. A membrane process to capture $\mathrm{CO}_{2}$ from coal-fired power plant flue gas, Projects NT43085 and NT05312, Prepared by Membrane Technology and Research, Inc. for NETL Review Meeting, DOE/NETL, Pittsburgh, PA, March 26. 2009.

[69] IEA. Energy Technology Perspective 2020. Paris, France. 2020.

[70] IEA. Global Energy Review 2020. The impacts of the Covid-19 crisis on global energy demand and $\mathrm{CO}_{2}$ emissions. Paris, France. 2020.

[71] IEA. Transforming Industry through CCUS. Paris, France. 2019.

[72] Fuel Cells and Hydrogen 2 Joint Undertaking. Hydrogen Roadmap Europe. Belgium, Brussels2019.

[73] Allied Market Research. Waste to Energy Market, by Technology: Global Opportunity Analysis and Industry Forecast, 2018 - 2025. Pune, India2018.

[74] Global CCS Institute. Global status of CCS 2019. 2019.

[75] European Commission. a strategic long-term vision for a prosperous, modern, competitive and climate neutral economy by 2050 - A Clean Planet for all. Brussels, Belgium2018.

[76] Global CCS Institute. CO2RE Facilities database. 2020.

[77] IEA. Putting $\mathrm{CO}_{2}$ to use. Paris, France. 2019.

[78] IEAGHG. Iron and steel CCS study (Techno-economic integrated steel mill). 2013/4. Cheltenham, United Kingdom. 2013.

[79] IEAGHG. Deployment of CCS in the Cement industry. 2013/19. Cheltenham, United Kingdom. 2013.

[80] Gardarsdottir S, De Lena E, Romano M, Roussanaly S, Voldsund M, Pérez-Calvo J-F, et al. Comparison of technologies for $\mathrm{CO}_{2}$ capture from cement production - Part 2: cost analysis. Energies. 2019;12:542.

[81] IEAGHG. Evaluating the Costs of Retrofitting $\mathrm{CO}_{2}$ Captured in an Integrated Oil Refinery. 2017/05. Cheltenham, United Kingdom. 2017.

[82] IEAGHG. Techno-economic evaluation of retroffitting CCS in a market pulp mill and an integrated pulp and board mill. 2016/10. Cheltenham, United Kingdom. 2016.

[83] IEAGHG. Techno-Economic Evaluation of Hyco Plant Integrated to Ammonia / Urea or Methanol Production with CCS. 2017/03. Cheltenham, United Kingdom. 2017.

[84] IEAGHG. $\mathrm{CO}_{2}$ Capture in Natural Gas Production by Adsorption Processes for $\mathrm{CO}_{2}$ Storage, EOR and EGR. 2017/04. Cheltenham, United Kingdom.2017.

[85] Roussanaly S, Aasen A, Anantharaman R, Danielsen B, Jakobsen J, Heme-De-Lacotte L, et al. Offshore power generation with carbon capture and storage to decarbonise mainland electricity and offshore oil and gas installations: A techno-economic analysis. Applied Energy. 2019;233-234:478-94.

[86] IEAGHG. Techno-economic evaluation of SMR based standalone (merchant) hydrogen plant with CCS. 2017/02. Cheltenham, United Kingdom. 2017.

[87] Leeson D, Mac Dowell N, Shah N, Petit C, Fennell PS. A Techno-economic analysis and systematic review of carbon capture and storage (CCS) applied to the iron and steel, cement, oil refining and pulp 
and paper industries, as well as other high purity sources. International Journal of Greenhouse Gas Control. 2017;61:71-84.

[88] IEAGHG. Cost of $\mathrm{CO}_{2}$ capture in the industrial sector: cement and iron and steel industries. 2018/03. Cheltenham, United Kingdom. 2018.

[89] Garcia M, Berghout N. Toward a common method of cost-review for carbon capture technologies in the industrial sector: cement and iron and steel plants. International Journal of Greenhouse Gas Control. 2019;87:142-58.

[90] Roussanaly S, Fu C, Voldsund M, Anantharaman R, Spinelli M, Romano M. Techno-economic Analysis of MEA $\mathrm{CO}_{2}$ Capture from a Cement Kiln - Impact of Steam Supply Scenario. Energy Procedia. 2017;114:6229-39.

[91] Jakobsen J, Roussanaly S, Anantharaman R. A techno-economic case study of $\mathrm{CO}_{2}$ capture, transport and storage chain from a cement plant in Norway. Journal of Cleaner Production. 2017;144:523-39.

[92] Bosoaga A, Masek O, Oakey JE. $\mathrm{CO}_{2}$ Capture Technologies for Cement Industry. Energy Procedia. 2009;1:133-40.

[93] IEAGHG. Understanding the cost of retrofitting $\mathrm{CO}_{2}$ capture in an integrated oil refineries. 2017/TR05. Cheltenham, United Kingdom. 2017.

[94] IEA. Tracking Clean energy Preogress report 2013. Paris, France. 2013.

[95] Global CCS Institute. CCS facilities Database. Available from: https://co2re.co/FacilityData. 2019.

[96] Nazir SM, Cloete JH, Cloete S, Amini S. Efficient hydrogen production with $\mathrm{CO}_{2}$ capture using gas switching reforming. Energy. 2019;185:372-85.

[97] International Association of Oil \& Gas Producers. Environmental performance indicators - 2016 data. 2016.

[98] Nord LO, Anantharaman R, Chikukwa A, Mejdell T. CCS on Offshore Oil and Gas Installation - Design of Post-Combustion Capture System and Steam Cycle. Energy Procedia. 2017;114:6650-9.

[99] Roussanaly S. Calculating $\mathrm{CO}_{2}$ avoidance costs of Carbon Capture and Storage from industry. Carbon Management. 2019;10:105-12.

[100] Deevski S. Cost Allocation Methods for Joint Products and By-products. Economic Alternatives. 2016:64-70.

[101] UNEP. Global Guidance Principles for life cycle assessment databases - A Basis for Greener Processes and Products, "Shonan Guidance Principles". 2011.

[102] Zimmermann AW, Wunderlich J, Müller L, Buchner GA, Marxen A, Michailos S, et al. TechnoEconomic Assessment Guidelines for CO2 Utilization. Frontiers in Energy Research. 2020;8.

[103] IEA. World energy outlook 2018. Paris, France. 2018.

[104] Berghout N, Meerman H, van den Broek M, Faaij A. Assessing deployment pathways for greenhouse gas emissions reductions in an industrial plant - A case study for a complex oil refinery. Applied Energy. 2019;236:354-78.

[105] Tanzer SE, Blok K, Ramírez A. Can bioenergy with carbon capture and storage result in carbon negative steel? International Journal of Greenhouse Gas Control. 2020;100:103104.

[106] Voldsund M, Gardarsdottir S, De Lena E, Pérez-Calvo J-F, Jamali A, Berstad D, et al. Comparison of technologies for $\mathrm{CO}_{2}$ capture from cement production - Part 1: technical evaluation. Energies. 2019;12:559.

[107] European Cement Research Academy. ECRA CCS Project: Report on Phase III, TR-ECRA-119/2012. Dusseldorf, Germany2012.

[108] Arasto A, Tsupari E, Kärki J, Pisilä E, Sorsamäki L. Post-combustion capture of $\mathrm{CO}_{2}$ at an integrated steel mill - Part I: Technical concept analysis. Int J Greenh Gas Con. 2013;16:271-7.

[109] Berghout N, van den Broek M, Faaij A. Techno-economic performance and challenges of applying $\mathrm{CO}_{2}$ capture in the industry: A case study of five industrial plants. International Journal of Greenhouse Gas Control. 2013;17:259-79. 
[110] Bureau-Cauchois G, Roussanaly S, Husebye J. Deliverable N D4.1.1 Economic Assessment of Flue Gas \& $\mathrm{CO}_{2}$ Collecting Networks. COCATE EU Project; 2011.

[111] Berghout N, Kuramochi T, Broek Mvd, Faaij A. Techno-economic performance and spatial footprint of infrastructure configurations for large scale $\mathrm{CO}_{2}$ capture in industrial zones: A case study for the Rotterdam Botlek area (part A). International Journal of Greenhouse Gas Control. 2015;39:256-84.

[112] Garđarsdóttir S, Normann F, Skagestad R, Johnsson F. Investment costs and $\mathrm{CO}_{2}$ reduction potential of carbon capture from industrial plants - A Swedish case study. International Journal of Greenhouse Gas Control. 2018;76:111-24.

[113] Cormos A-M, Cormos C-C. Reducing the carbon footprint of cement industry by post-combustion $\mathrm{CO}_{2}$ capture: Techno-economic and environmental assessment of a CCS project in Romania. Chemical Engineering Research and Design. 2017;123:230-9.

[114] AspenTech. Aspen Icarus Reference Guide. Burlington 2010.

[115] IEAGHG. $\mathrm{CO}_{2}$ capture in the cement industry. 2008/3. Cheltenham, United Kingdom. 2008.

[116] Deng $\mathrm{H}$, Roussanaly $\mathrm{S}$, Skaugen $\mathrm{G}$. Techno-economic analyses of $\mathrm{CO}_{2}$ liquefaction: Impact of product pressure and impurities. International Journal of Refrigeration. 2019;103:301-15.

[117] Skaugen G, Roussanaly S, Jakobsen J, Brunsvold A. Techno-economic evaluation of the effects of impurities on conditioning and transport of $\mathrm{CO}_{2}$ by pipeline. International Journal of Greenhouse Gas Control. 2016;54, Part 2:627-39.

[118] Sundqvist M, Biermann M, Normann F, Larsson M. Evaluation of Low and High Level of Integration Options for Carbon Capture at an Integrated Iron and Steel Mill. The 9th Tronheim conference on $\mathrm{CO}_{2}$ capture, transport and storage. Trondheim, Norway2017.

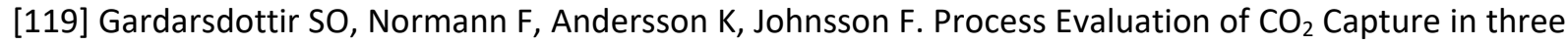
Industrial case Studies. Energy Procedia. 2014;63:6565-75.

[120] Zero Emission Platform. The costs of $\mathrm{CO}_{2}$ transport, Post-demonstration CCS in the EU2011.

[121] Zero Emission Platform. The costs of $\mathrm{CO}_{2}$ storage, post-demonstration CCS in the EU 2011.

[122] Rubin ES, Davison JE, Herzog HJ. The cost of $\mathrm{CO}_{2}$ capture and storage. International Journal of Greenhouse Gas Control. 2015;40:378-400.

[123] Roussanaly S, Anantharaman R. Cost-optimal $\mathrm{CO}_{2}$ capture ratio for membrane-based capture from different $\mathrm{CO}_{2}$ sources. Chemical Engineering Journal. 2017;327:618-28.

[124] Roussanaly S, Jakobsen JP, Hognes EH, Brunsvold AL. Benchmarking of $\mathrm{CO}_{2}$ transport technologies: Part I-Onshore pipeline and shipping between two onshore areas. International Journal of Greenhouse Gas Control. 2013;19:584-94.

[125] Roussanaly S, Brunsvold AL, Hognes ES. Benchmarking of $\mathrm{CO}_{2}$ transport technologies: Part II Offshore pipeline and shipping to an offshore site. International Journal of Greenhouse Gas Control. 2014;28:283-99.

[126] Knoope MMJ, Guijt W, Ramírez A, Faaij APC. Improved cost models for optimizing $\mathrm{CO}_{2}$ pipeline configuration for point-to-point pipelines and simple networks. International Journal of Greenhouse Gas Control. 2014;22:25-46.

[127] Roussanaly S, Grimstad A-A. The Economic Value of $\mathrm{CO}_{2}$ for EOR Applications. Energy Procedia. 2014;63:7836-43.

[128] IHS. The IHS upstream costs indexes.2018.

[129] Roussanaly S, Skaugen G, Aasen A, Jakobsen J, Vesely L. Techno-economic evaluation of $\mathrm{CO}_{2}$ transport from a lignite-fired IGCC plant in the Czech Republic. International Journal of Greenhouse Gas Control. 2017;65:235-50.

[130] Roussanaly S, Bureau-Cauchois $\mathrm{G}$, Husebye J. Costs benchmark of $\mathrm{CO}_{2}$ transport technologies for a group of various size industries. International Journal of Greenhouse Gas control. 2013;12C:341-50. 
[131] Aspelund A, Mølnvik MJ, De Koeijer G. Ship Transport of $\mathrm{CO}_{2}$ : Technical Solutions and Analysis of Costs, Energy Utilization, Exergy Efficiency and $\mathrm{CO}_{2}$ Emissions. Chemical Engineering Research and Design. 2006;84:847-55.

[132] Durusut E, Joos M. Shipping $\mathrm{CO}_{2}$ - UK Cost Estimation Study. 2018.

[133] Berghout N, van den Broek M, Faaij A. Deployment of infrastructure configurations for large-scale $\mathrm{CO}_{2}$ capture in industrial zones: A case study for the Rotterdam Botlek area (part B). International Journal of Greenhouse Gas Control. 2017;60:24-50.

[134] Porter RTJ, Mahgerefteh H, Brown S, Martynov S, Collard A, Woolley RM, et al. Techno-economic assessment of $\mathrm{CO}_{2}$ quality effect on its storage and transport: $\mathrm{CO}_{2} \mathrm{QUEST}$ : An overview of aims, objectives and main findings. International Journal of Greenhouse Gas Control. 2016;54, Part 2:662-81.

[135] Porter RTJ, Fairweather M, Kolster C, Mac Dowell N, Shah N, Woolley RM. Cost and performance of some carbon capture technology options for producing different quality $\mathrm{CO}_{2}$ product streams. International Journal of Greenhouse Gas Control. 2017;57:185-95.

[136] Brunsvold A, Jakobsen JP, Mazzetti MJ, Skaugen G, Hammer M, Eickhoff C, et al. Key findings and recommendations from the IMPACTS project. International Journal of Greenhouse Gas Control. 2016;54, Part 2:588-98.

[137] McCoy ST. The Economics of $\mathrm{CO}_{2}$ Transport by Pipeline and Storage in Saline Aquifers and Oil Reservoirs. Carnegie Melon Univerisity, Department of Engineering and Public Policy.; 2009.

[138] Wei N, Li X, Wang Q, Gao S. Budget-type techno-economic model for onshore $\mathrm{CO}_{2}$ pipeline transportation in China. International Journal of Greenhouse Gas Control. 2016;51:176-92.

[139] Shogenova A, Piessens K, Holloway S, Bentham M, Martínez R, Flornes KM, et al. Implementation of the EU CCS Directive in Europe: Results and Development in 2013. Energy Procedia. 2014;63:6662-70.

[140] Roussanaly S, Straus J, Anantharaman R., Meyer O. Developing an infrastructure to deliver H2 to Europe and the Norwegian market. Webinar: Hydrogen from Norwegian Natural Gas to Decarbonise Europe \& Norway, 24th June 2020.

[141] AACEl. Cost Estimate Classification System - As Applied in Engineering, Procurement, and Construction for the Process Industries, AACE International Recommended Practice No. 18R-97 (revised June 15, 1998). www.aacei.org. 1997.

[142] EPRI. Technology Assessment Guide. Palo Alto, CA. EPRI TR-102276-V1R7. . 1993.

[143] Rubin ES, Mantripragada H, Marks A, Versteeg P, Kitchin J. The outlook for improved carbon capture technology. Progress in Energy and Combustion Science. 2012;38:630-71.

[144] Bakhtiary-Davijany $\mathrm{H}$, Myhrvold T. On Methods for Maturity Assessment of $\mathrm{CO}_{2}$ Capture Technologies. Energy Procedia. 2013;37:2579-84.

[145] Neele F, Wolf M, Kapetaki Z, Wildenborg T. Future CCS Technologies, European Zero Emission Technology and Innovation Platform. 2017.

[146] Freeman BC, Bhown AS. Assessment of the technology readiness of post-combustion $\mathrm{CO}_{2}$ capture technologies. Energy Procedia. 2011;4:1791-6.

[147] Knaggs M, Ramsey J, Unione A, Harkreader D, Oelfke J, Keairns D, et al. Application of Systems Readiness Level Methods in Advanced Fossil Energy Applications. Procedia Computer Science. 2015;44:497-506.

[148] IEAGHG. Further assessments of emerging $\mathrm{CO}_{2}$ capture technologies for the power sector and their potential to reduce costs. 2019/09. Cheltenham, United Kingdom. 2019.

[149] CLEANKER project. CLEAN clinKER by calcium looping for low- $\mathrm{CO}_{2}$ cement. Available from: http://www.cleanker.eu/the-project/objectives.html. 2020.

[150] STEPWISE project. The stepwise project. Available from: https://www.stepwise.eu/. 2020.

[151] LEILAC project. The LEILAC pilot plant. Available from: https://www.project-leilac.eu/leilac-pilotplant. 2020. 
[152] Jordal K, Abanades C, Cinti G, Berstad B, Hoening V, Homberger M, et al. CEMCAP D2.11 CEMCAP Strategic conclusions - progressing $\mathrm{CO}_{2}$ capture from cement towards demonstration, Trondheim, Norway. 2019.

[153] van der Spek M, Ramirez A, Faaij A. Improving uncertainty evaluation of process models by using pedigree analysis. A case study on $\mathrm{CO}_{2}$ capture with monoethanolamine. Computers \& Chemical Engineering. 2016;85:1-15.

[154] Harenberg D, Marelli S, Sudret B, Winschel V. Uncertainty quantification and global sensitivity analysis for economic models. Quantitative Economics. 2019;10:1-41.

[155] Borgonovo E, Plischke E. Sensitivity analysis: A review of recent advances. European Journal of Operational Research. 2016;248:869-87.

[156] Pohjola MV, Pohjola P, Tainio M, Tuomisto JT. Perspectives to performance of environment and health assessments and models--from outputs to outcomes? Int J Environ Res Public Health. 2013;10:2621-42.

[157] van der Spek M, Sanchez Fernandez E, Eldrup NH, Skagestad R, Ramirez A, Faaij A. Unravelling uncertainty and variability in early stage techno-economic assessments of carbon capture technologies. International Journal of Greenhouse Gas Control. 2017;56:221-36.

[158] Raksajati A, Ho MT, Wiley DE. Comparison of Solvent Development Options for Capture of $\mathrm{CO}_{2}$ from Flue Gases. Industrial \& Engineering Chemistry Research. 2018;57:6746-58.

[159] Hanak DP, Manovic V. Economic feasibility of calcium looping under uncertainty. Applied Energy. 2017;208:691-702.

[160] van der Spek M, Ramirez A. A Structured Approach for Selecting Carbon Capture Process Models. A Case Study on Monoethanolamine. Energy Procedia. 2014;63:1287-95.

[161] Razi N, Svendsen HF, Bolland O. Assessment of mass transfer correlations in rate-based modeling of a large-scale $\mathrm{CO}_{2}$ capture with MEA. International Journal of Greenhouse Gas Control. 2014;26:93-108.

[162] Fosbøl PL, Gaspar J, Ehlers S, Kather A, Briot P, Nienoord M, et al. Benchmarking and Comparing First and Second Generation Post Combustion $\mathrm{CO}_{2}$ Capture Technologies. Energy Procedia. 2014;63:27-44.

[163] DOE/NETL. Quality guidelines for energy system studies Performing a Techno-economic Analysis for Power Generation Plants. Pittsburgh, PA. 2015.

[164] Cesar. Deliverable D2.4.3. European Best Practice Guidelines for Assessment of $\mathrm{CO}_{2}$ Capture Technologies. Delft, the Netherlands. 2011.

[165] IEAGHG. Criteria for Technical and Economic Assessment of Plants With Low $\mathrm{CO}_{2}$ Emissions. 2009/TR 03. Cheltenham, United Kingdom. 2009.

[166] Zero Emission Platform. The costs of $\mathrm{CO}_{2}$ capture, Post-demonstration CCS in the EU2011.

[167] Roy CJ, Oberkampf WL. A comprehensive framework for verification, validation, and uncertainty quantification in scientific computing. Computer Methods in Applied Mechanics and Engineering. 2011;200:2131-44.

[168] Swiler L, Paez T, Mayes R, Eldred M. Epistemic Uncertainty in the Calculation of Margins. $50^{\text {th }}$ AIAA/ASME/ASCE/AHS/ASC Structures, Structural Dynamics, and Materials Conference.

[169] Ascough JC, Maier HR, Ravalico JK, Strudley MW. Future research challenges for incorporation of uncertainty in environmental and ecological decision-making. Ecological Modelling. 2008;219:383-99.

[170] Sigel K, Klauer B, Pahl-Wostl C. Conceptualising uncertainty in environmental decision-making: The example of the EU water framework directive. Ecological Economics. 2010;69:502-10.

[171] Walker WE, Harremoës P, Rotmans J, van der Sluijs JP, van Asselt MBA, Janssen P, et al. Defining Uncertainty: A Conceptual Basis for Uncertainty Management in Model-Based Decision Support. Integrated Assessment. 2003;4:5-17.

[172] Knol AB, Petersen AC, van der Sluijs JP, Lebret E. Dealing with uncertainties in environmental burden of disease assessment. Environmental Health. 2009;8:21. 
[173] Van Der Sluijs JP, Craye M, Funtowicz S, Kloprogge P, Ravetz J, Risbey J. Combining Quantitative and Qualitative Measures of Uncertainty in Model-Based Environmental Assessment: The NUSAP System. Risk Analysis. 2005;25:481-92.

[174] Saltelli A, Ratto M, Andres T, Campolongo F, Cariboni J, Gatelli D, et al. Global Sensitivity Analysis. The Primer: Chichester: John Wiley \& Sons Ltd.; 2007.

[175] Saltelli A, Tarantola S, Campolongo F, Ratto M. Sensitivity Analysis.: Chichester: John Wiley \& Sons Ltd.; 2004.

[176] Sudret B. Global sensitivity analysis using polynomial chaos expansions. Reliability Engineering \& System Safety. 2008;93:964-79.

[177] IEA. Energy Technology Perspectives 2014. Paris, France. 2014.

[178] Mikunda T, van Deurzen J, Seebregts A, Kerssemakers K, Tetteroo M, Buit L. Towards a $\mathrm{CO}_{2}$ infrastructure in North-Western Europe: Legalities, costs and organizational aspects. Energy Procedia. 2011;4:2409-16.

[179] Zhai H, Rubin ES. Comparative Performance and Cost Assessments of Coal- and Natural-Gas-Fired Power Plants under a $\mathrm{CO}_{2}$ Emission Performance Standard Regulation. Energy \& Fuels. 2013;27:4290-301. [180] Hawer S, Schönmann A, Reinhart G. Guideline for the Classification and Modelling of Uncertainty and Fuzziness. Procedia CIRP. 2018;67:52-7.

[181] Mishra S, Datta-Gupta A. Chapter 6 - Uncertainty Quantification. In: Mishra S, Datta-Gupta A, editors. Applied Statistical Modeling and Data Analytics: Elsevier; 2018. p. 119-67.

[182] Kleijnen J. Design and Analysis of Simulation Experiments (International Series in Operations Research \& Management Science): 2nd ed. Heidelberg: Springer.; 2010.

[183] Ravetz J, Funtowicz S. Uncertainty and quality in science for policy: Wolters Kluwer; 1990.

[184] Fernández-Dacosta C, van der Spek M, Hung CR, Oregionni GD, Skagestad R, Parihar P, et al. Prospective techno-economic and environmental assessment of carbon capture at a refinery and $\mathrm{CO}_{2}$ utilisation in polyol synthesis. Journal of $\mathrm{CO}_{2}$ Utilization. 2017;21:405-22.

[185] Mendoza Beltran A, Heijungs R, Guinée J, Tukker A. A pseudo-statistical approach to treat choice uncertainty: the example of partitioning allocation methods. The International Journal of Life Cycle Assessment. 2016;21:252-64.

[186] Bailera M, Hanak DP, Lisbona P, Romeo LM. Techno-economic feasibility of power to gas-oxy-fuel boiler hybrid system under uncertainty. International Journal of Hydrogen Energy. 2019;44:9505-16.

[187] Sun AY, Jeong H, González-Nicolás A, Templeton TC. Metamodeling-based approach for risk assessment and cost estimation: Application to geological carbon sequestration planning. Computers \& Geosciences. 2018;113:70-80.

[188] Morgan JC, Bhattacharyya D, Tong C, Miller DC. Uncertainty quantification of property models: Methodology and its application to $\mathrm{CO}_{2}$-loaded aqueous MEA solutions. AIChE Journal. 2015;61:1822-39. [189] Morgan JC, Chinen AS, Omell B, Bhattacharyya D, Tong C, Miller DC. Thermodynamic modeling and uncertainty quantification of $\mathrm{CO}_{2}$-loaded aqueous MEA solutions. Chemical Engineering Science. 2017;168:309-24.

[190] Soares Chinen A, Morgan JC, Omell B, Bhattacharyya D, Tong C, Miller DC. Development of a Rigorous Modeling Framework for Solvent-Based $\mathrm{CO}_{2}$ Capture. 1. Hydraulic and Mass Transfer Models and Their Uncertainty Quantification. Industrial \& Engineering Chemistry Research. 2018;57:10448-63.

[191] Morgan JC, Soares Chinen A, Omell B, Bhattacharyya D, Tong C, Miller DC, et al. Development of a Rigorous Modeling Framework for Solvent-Based $\mathrm{CO}_{2}$ Capture. Part 2: Steady-State Validation and Uncertainty Quantification with Pilot Plant Data. Industrial \& Engineering Chemistry Research. 2018;57:10464-81.

[192] Tong C. PSUADE Reference Manual (Version 1.7). Lawrence Livermore National Laboratory, Livermore, CA. 
[193] Sobol IM. Global sensitivity indices for nonlinear mathematical models and their Monte Carlo estimates. Mathematics and Computers in Simulation. 2001;55:271-80.

[194] Friedman JH. Multivariate Adaptive Regression Splines. The Annals of Statistics. 1991;19:1-67, .

[195] Chaloner K, Verdinelli I. Bayesian Experimental Design: A Review. Statistical Science. 1995;10:273304, 32.

[196] Kimaev G, Ricardez-Sandoval LA. Multilevel Monte Carlo applied to chemical engineering systems subject to uncertainty. AIChE Journal. 2018;64:1651-61.

[197] Soepyan FB, Anderson-Cook CM, Morgan JC, Tong CH, Bhattacharyya D, Omell BP, et al. Sequential Design of Experiments to Maximize Learning from Carbon Capture Pilot Plant Testing. In: Eden MR, lerapetritou MG, Towler GP, editors. Computer Aided Chemical Engineering: Elsevier; 2018. p. 283-8.

[198] Morgan JC, Chinen AS, Anderson-Cook C, Tong C, Carroll J, Saha C, et al. Development of a framework for sequential Bayesian design of experiments: Application to a pilot-scale solvent-based $\mathrm{CO}_{2}$ capture process. Applied Energy. 2020;262:114533.

[199] Myers R, Montgomery D, Anderson-Cook C. Practical Design Optimality

Response Surface Methodology: Process and Product Optimization using Designed Experiments, 4th ed. New York: Wiley; 2016. p. 467-74.

[200] Morgan J, Omell B, Matuszewski M, Miller DC, Tong C, Brenda N, et al. Application of Sequential Design of Experiments (SDoE) to a Pilot-Scale MEA-Based $\mathrm{CO}_{2}$ Capture Process. The 10th Trondheim Conference on $\mathrm{CO}_{2}$ Capture, Transport, and Storage. Trondheim. 2019.

[201] Maußner J, Freund $\mathrm{H}$. Multi-objective reactor design under uncertainty: A decomposition approach based on cubature rules. Chemical Engineering Science. 2020;212:115304.

[202] Wang K, Chen J, Xie L, Su H. Decision making scheme of integration design and control under uncertainty for enhancing the economic performance of chemical processes with multiplicity behaviors. Chemical Engineering Research and Design. 2019;150:327-40.

[203] Khajuria H, Pistikopoulos EN. Optimization and Control of Pressure Swing Adsorption Processes Under Uncertainty. AIChE Journal. 2013;59:120-31.

[204] Gebreslassie BH, Guillén-Gosálbez G, Jiménez L, Boer D. Economic performance optimization of an absorption cooling system under uncertainty. Applied Thermal Engineering. 2009;29:3491-500.

[205] Cerrillo-Briones IM, Ricardez-Sandoval LA. Robust optimization of a post-combustion $\mathrm{CO}_{2}$ capture absorber column under process uncertainty. Chemical Engineering Research and Design. 2019;144:38696.

[206] Bjerketvedt VS, Tomasgard A, Roussanaly S. Optimal design and cost of ship-based $\mathrm{CO}_{2}$ transport under uncertainties and fluctuations. International Journal of Greenhouse Gas Control. 2020;103:103190. [207] Knoope MMJ, Ramírez A, Faaij APC. The influence of uncertainty in the development of a $\mathrm{CO}_{2}$ infrastructure network. Applied Energy. 2015;158:332-47.

[208] Roussanaly S, Ouassou JA, Anantharaman R, Haaf M. Impact of Uncertainties on the Design and Cost of CCS From a Waste-to-Energy Plant. Frontiers in Energy Research. 2020;8.

[209] Sugiyama S. Monte carlo simulation/risk analysis on a spreadsheet: review of three software packages. Foresight. 2008;9:36-42.

[210] LLC SD. RiskAMP User Guide. User Guide for the RiskAMP Monte Carlo Add-in. 2012.

[211] Charnes J. Financial Modeling with Crystal Ball and Excel: Hoboken: John Wiley \& Sons Ltd.; 2007.

[212] Swiler L. Approaches for representing and propagating uncertainty that will be useful for multi-scale modeling, in SNL-GT materials workshop. 2016.

[213] Marelli S, Sudret B. UQLab: A Framework for Uncertainty Quantification in Matlab. Vulnerability, Uncertainty, and Risk2014. p. 2554-63.

[214] Miller DC, Ng B, Eslick J, Tong C, Chen Y. Advanced Computational Tools for Optimization and Uncertainty Quantification of Carbon Capture Processes. In: Eden MR, Siirola JD, Towler GP, editors. Computer Aided Chemical Engineering: Elsevier; 2014. p. 202-11. 
[215] Larson ED, Kreutz TG, Greig C, Williams RH, Rooney T, Gray E, et al. Design and analysis of a lowcarbon lignite/biomass-to-jet fuel demonstration project. Applied Energy. 2020;260:114209.

[216] Platts. HVAC: Centrifugal chillers (accessed 13.07.11). Available from https://www.reliant.com/enUS/Platts/PDF/P_PA_14.pdf. 2004.

[217] Rao AB, Rubin ES. A Technical, Economic, and Environmental Assessment of Amine-Based $\mathrm{CO}_{2}$ Capture Technology for Power Plant Greenhouse Gas Control. Environmental Science \& Technology. 2002;36:4467-75.

[218] Zhuang Q. Comparative kinetics of ammonia-based $\mathrm{CO}_{2}$ capture and amine $\mathrm{CO}_{2}$ capture technologies: absorber sizing. $10^{\text {th }}$ Annual Conference on Carbon Capture \& Sequestration, Pittsburgh, PA, USA, May 2-5, 2011. 2011.

[219] Berkenpas M, Frey H, Fry J, Kalagnanam J, Rubin E. Technical Documentation: Integrated Environmental Control Model (accessed16.05.11). Available from http://www.cmu.edu/epp/iecm/IECMPublications/a\%20Berkenpas\%20et\%20al,\%20IECM\%20Tech.pdf. 1999.

[220] Rubin E, Zhai H. The cost of CCS for natural gas-fired power plants. $10^{\text {th }}$ Annual Conference on Carbon Capture and Storage, Pittsburgh, PA, USA, May 2-5, 2011. 2011.

[221] EIA. Annual Energy Outlook 2012, Energy Information Administration. U.S. Department of Energy, Washington, DC. Available at: $h t t p: / / w w w . e i a . g o v / f o r e c a s t s / a e o / p d f / 0383(2012) . p d f .2012$.

[222] Anantharaman R, Fu C, Roussanaly S, Volsund M. D4.2 Design and performance of CEMCAP cement plant with MEA post combustion capture. Trondheim, Norway: CEMCAP EU Project; 2016.

[223] Roussanaly S, Alejo Vargas L, Anantharaman R, Gladysz P, Nazir SM. Enabling climate-positive industrial sectors - a cost-efficient approach. 15th International virtual conference on Greenhouse Gas Control Technologies. 15-18th March 2021. 2021.

[224] Tanzer SE, Blok K, Ramirez A. Negative emissions in the chemical sector: lifecycle $\mathrm{CO} 2$ accounting for biomass and CCS integration into ethanol, ammonia, urea, and hydrogen production. 15th International virtual conference on Greenhouse Gas Control Technologies. 15-18th March 2021. 2021.

[225] Ho M, Allinson G, Wiley D. Comparison of MEA capture cost for low $\mathrm{CO}_{2}$ emissions sources in Australia. International Journal of Greenhouse Gas Control. 2011;5:49-60.

[226] Kuramochi T, Ramírez A, Turkenburg W, Faaij A. Comparative assessment of $\mathrm{CO}_{2}$ capture technologies for carbon-intensive industrial processes. Progress in Energy and Combustion Science. 2012;38:87-112.

[227] European commission. HORIZON 2020 -- WORK PROGRAMME 2014-2015 General Annexes, European Commission. Brussels, Belgium. 2014.

[228] UK Ministry of Defence. Guidance - Standard terms and conditions. Available from: https://www.gov.uk/guidance/defence-and-security-accelerator-terms-and-conditions-and-contractguidance. 2016.

[229] NASA. Technology Readiness Level. Available from: https://www.nasa.gov/directorates/heo/scan/engineering/technology/txt_accordion1.html. Accessed on 16 December 2020. 2012.

[230] IEA. Clean Energy Innovation. Paris, France. 2020. 